\title{
Matched Filtering in Massive MU-MIMO Systems
}

by

Shuang Li

\author{
A thesis \\ submitted to Victoria University of Wellington \\ in fulfilment of the \\ requirements for the degree of \\ Doctor of Philosophy \\ in Engineering. \\ Victoria University of Wellington \\ 2020
}





\begin{abstract}
This thesis considers the analysis of matched filtering (MF) processing in massive multi-user multiple-input-multiple-output (MU-MIMO) wireless communication systems. The main focus is the analysis of system performance for combinations of two linear processers, analog maximum ratio combining (MRC) and digital MRC. We consider implementations of these processing techniques both at a single base-station (BS) and in distributed BS layouts. We further consider extremely low complexity distributed variants of MRC for such systems. Since MRC relies on the massive MIMO properties of favourable propagation (FP) and channel hardening, we also present a detailed analysis of FP and channel hardening. This analysis employs modern ray-based models rather than classical channel models as the models are more reliable for the large arrays and higher frequencies envisaged for future systems.

The importance of MRC processing is being driven by the emergence of massive MIMO and millimetre wave as strong candidates for next generation wireless communication systems. Massive MIMO explores the spatial dimension by providing significant increases in data rate, link reliability and energy efficiency. However, with a large number of antennas co-located in a fixed physical space, correlation between the elements of antennas may have a negative impact. Distributed systems, where the total number of antennas are divided into different locations, make this problem less serious. Also, linear processing techniques, analog MRC and digital MRC, due to their simplicity and efficiency, are more practical in massive MU-MIMO systems. For
\end{abstract}


these reasons we consider MRC processing in both co-located and distributed scenarios.

Although distributed systems reduce the adverse impact of correlation caused by closely-spaced large antenna arrays by dividing the antennas into multiple antenna clusters, the correlation within the cluster still exists. Thus, we extend MRC analysis for massive MIMO to correlated channels. Approximations of expected per-user spectrum efficiency (SE) with correlation effects for massive MIMO systems with analog MRC and digital MRC are derived. Useful insights are given for future system deployments. A convergence analysis of the interference behaviour under different correlation models is presented.

Furthermore, a distributed fully cooperative system, where all the received signals are sent to the central processor, offers attractive performance gains but at the cost of high computational complexity at the central node. Thus, we propose four low-complexity, two-stage processors, where only processed signals after local processing (first-stage) are transmitted to the global processing node (second-stage). We present analytical expressions for the expected per user SINR in an uplink distributed MU-MIMO system with twostage beamforming. This leads to an approximation of expected per-user SE.

The analysis of both millimetre wave and massive MIMO systems requires a strong link to the physical environment and ray-based models are more practical and suitable for such systems. However, it is unclear how the key properties in conventional MIMO systems, such as FP and channel hardening, will behave in a ray-based channel model. In this thesis, remarkably simple and general results are obtained demonstrating that: a) channel hardening may or may nor occur depending on the nature of the channel models; b) FP is guaranteed for all models as long as the ray angles are continuous random variables; c) we also propose a novel system metric, denoted large system po- 
tential (LSP) as the ratio of the mean desired signal power to the total mean interference power, where both the numbers of antennas and end-users are growing to infinity at a fixed ratio. We derive simple approximations to LSP and demonstrate that LSP will not normally hold as the mean interference power usually grows logarithmically relative to the mean signal power. 


\section{Statement of Originality}

The research presented in this thesis has not been submitted for another degree in any other university or educational institution.

The work presented in this thesis is the result of original research carried out by myself, in collaboration with my supervisors, Prof. Peter J. Smith (Professor of Statistics, Victoria University of Wellington, New Zealand) and Assoc. Prof. Pawel A. Dmochowski (Associate Professor of Engineering and Computer Science, Victoria University of Wellington, New Zealand).

The substantial majority of this work is my own.

Shuang Li

School of Engineering and Computer Science

Victoria University of Wellington, New Zealand

March 12, 2020 


\section{Acknowledgements}

This work is dedicated to the author's grandmother, Xinlan Li, who brought up Shuang and passed away during Shuang's PhD journey.

The author would like to say a big thank you to Assoc. Prof. Pawel A. Dmochowski for having the opportunity to undertake postgraduate study under the topic of wireless communications. Assoc. Prof. Pawel A. Dmochowski has been a fantastic mentor to guide the author to overcome all the difficulties arising from this journey. At the same time Assoc. Prof. Pawel A. Dmochowski has also pushed the author further with strict principles to learn more. Thanks, Assoc. Prof. Pawel A. Dmochowski, for being so encouraging and doing everything you can to keep the author motivated and supported. Without your support, effort, and feedback, the author would not have come so far.

Shuang would also like to give her heartiest gratitude to Prof. Peter J. Smith for his unlimited patience, on-going encouragement, and invaluable technical support. He has led Shuang to discover the beauty of the generality, elegance, and simplicity of mathematics, from the details and special cases to more general theorems. The biggest contribution of this work is just as David Hilbert put it "The art of doing mathematics is finding that special 
viii

case that contains all the germs of generality." This research has benefited a lot from Prof. Peter J. Smith's unique skill of combining wireless communication theory with mathematical analysis. He has always been there to answer Shuang's innumerable questions related to the research. The most valuable thing Shuang has learned from Prof. Peter J. Smith is that industriousness, patience, and endurance are much more important than anything else.

Sincere appreciation also goes to Dr. Callum Thomas Neil, Dr. Jawad Mirza, Dr. Muhammad Ali Raza Anjum and Dr. Steven Van Kuyk for helping the author to understand some critical concepts at the beginning of the research and providing useful suggestions on starting a life in a new foreign country.

Finally, the author would like to express her deepest gratitude to her family for their unconditional love, support, and sacrifice. 
1 Introduction 1

1.1 Motivation ..................... 3

1.1.1 Low-complexity Beamforming (BF) Analysis in Distributed Systems ....................... 4

1.1.2 Spatial Correlation for Distributed and Co-located Sys-

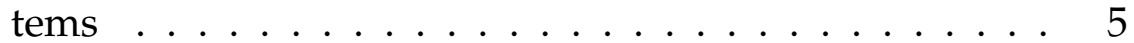

1.1.3 Distributed Hybrid MRC in Two Stage MU-MIMO Sys-

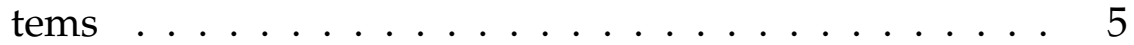

1.1.4 Asymptotic Behaviour for Distributed Systems . . . . . . 6

1.1.5 Massive MIMO for Ray-based Channels . . . . . . . . . . 7

1.2 Contributions ................... 8

1.2.1 Analysis of Analog MRC for Distributed MU-MIMO Systems $($ Chapter 4$) \ldots \ldots \ldots$

1.2.2 Analysis of Analog and Digital MRC in Massive MUMIMO Systems over Correlated Channels (Chapter 5) . 9

1.2.3 Hybrid MRC with Imperfect CSI in Two Stage Distributed MU-MIMO Systems (Chapter 6) . . . . . . . . . . 10 
1.2.4 Massive MIMO Asymptotics for Ray-Based Channels (Chapter 7) . . . . . . . . . . . . 10

1.3 Thesis Outline . . . . . . . . . . . . . . . . . . . . 11

1.4 Publications . . . . . . . . . . . . . . . . 12

2 MU-MIMO Channel Modelling $\quad 15$

2.1 The Propagation Properties of Wireless Communication Channels ........................... 16

2.1.1 Distance-Based Path Loss and Shadowing . . . . . . . 16

2.1.2 Blockage and Outage . . . . . . . . . . . . 17

2.2 Statistical Channel Models . . . . . . . . . . . . . . . . . . . . 18

2.2 .1 I.I.D Rayleigh . . . . . . . . . . . . . . . . . . . 18

2.2 .2 I.I.D Ricean . . . . . . . . . . . . . . . . . . 18

2.3 Narrowband MIMO Channel . . . . . . . . . . . . . . . . . . . . 19

2.3.1 Narrowband Single-user MIMO Channel . . . . . . . . . 19

2.3.2 Narrowband Multi-user MIMO Channel . . . . . . . . . 20

2.4 Ray-based MIMO Channel Models . . . . . . . . . . . . . . . . . 21

2.5 Spatial Correlation . . . . . . . . . . . . . . . . . 24

2.5 .1 Exponential ...................... 26

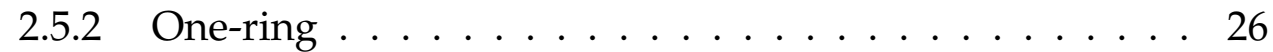

2.5.3 Correlated MIMO Channel . . . . . . . . . . . . . 27

2.6 Channel State Information (CSI) . . . . . . . . . . . . . 27

2.6.1 Feedback .................... 28

2.6 .2 Reciprocity .................. 28

2.7 Massive MIMO Properties . . . . . . . . . . . . . . . . . . . 28

3 System Architectures and Beamforming Techniques For Massive MUMIMO Wireless Communications 31

3.1 Analog BF Architecture . . . . . . . . . . . . . . . 32 
3.2 Digital BF Architecture . . . . . . . . . . . . . . . . 33

3.3 Hybrid Analog-Digital Precoding Architecture . . . . . . . . 35

3.4 The Relationship between Analog, Digital, and Hybrid BF . . 36

3.5 Linear Beamforming . . . . . . . . . . . . . . . . . . 38

3.5.1 Single-user MIMO Beamforming . . . . . . . . . . . . 38

3.5.2 Multi-user MIMO Beamforming . . . . . . . . . . . 39

3.5 .3 Spectral Efficiency $\ldots \ldots \ldots \ldots$. . . . . . . 40

4 Analysis of Analog MRC for Distributed MU-MIMO Systems 41

4.1 Introduction . . . . . . . . . . . . . . . . . . . 41

4.2 System Model . . . . . . . . . . . . . . . . . . . 44

4.2.1 Uplink System Model . . . . . . . . . . . . . . . . . . 45

4.2.2 Downlink System Model … . . . . . . . . . . . 48

4.3 Approximations of Achievable Uplink and Downlink Spectral Efficiency . . . . . . . . . . . . . . . . . . . 48

$4.3 .1 \quad$ Expected Signal Power . . . . . . . . . . . . . . . 50

4.3.2 Expected Interference Power . . . . . . . . . . . . 52

4.4 Numerical Results . . . . . . . . . . . . . . . . . . . . . . . . 55

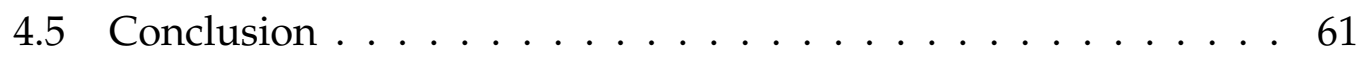

5 Analysis of Analog and Digital MRC in Massive MU-MIMO Systems over Correlated Channels 63

5.1 Introduction . . . . . . . . . . . . . . . . . . 64

5.2 System Model . . . . . . . . . . . . . . . . . . . . . . 66

5.3 Asymptotic SINR Analysis . . . . . . . . . . . . . . . . . . . 68

5.3.1 Asymptotic analysis for i.i.d. Rayleigh fading . . . . . 69

5.3.2 Asymptotic analysis, perfect correlation, equal matrices 71

5.3.3 Asymptotic analysis, perfect correlation, unequal matrices . . . . . . . . . . . . . . 71 
5.3.4 Analog MRC correlation analysis . . . . . . . . . . 74

5.4 Numerical Results . . . . . . . . . . . . . . . . 76

5.5 Conclusion . . . . . . . . . . . . . . . . 80

6 Hybrid MRC $\quad 83$

6.1 Introduction . . . . . . . . . . . . 84

6.2 System Model . . . . . . . . . . . . . . . . . . . . . . 85

6.3 Approximations of Achievable Uplink Spectral Efficiency . . . . 86

6.3.1 Two-stage SINR for A-D and D-D . . . . . . . . . 87

6.3.2 Asymptotic Analysis of A-D and D-D . . . . . . . . . 91

$6.3 .3 \mathrm{~A}-\mathrm{ZF}$ and D-ZF SINR . . . . . . . . . . . . 92

6.4 Numerical Results . . . . . . . . . . . . . . . . . . . . . . . . 93

6.5 Conclusion . . . . . . . . . . . . . . . . . . 97

7 Massive MIMO for Ray-Based Channels 99

7.1 Introduction . . . . . . . . . . . . . . . . . 100

7.2 Channel Model and System Metrics . . . . . . . . . . . . 103

7.2.1 Ray-based Channel Model . . . . . . . . . . . . . . . 104

7.2.2 FP, Channel Hardening and Large System Potential . . . 106

7.3 ULA: Channel Hardening, FP and LSP . . . . . . . . . . . . . 107

7.3.1 Channel Hardening . . . . . . . . . . . . 107

7.3.2 FP (Single-Sided Massive MIMO) . . . . . . . . . . . 108

7.3.3 Large System Potential (Double-Sided Massive MIMO) . 109

7.3.4 Avoiding Interference Growth . . . . . . . . . . . . . 114

7.4 UPA: Channel Hardening, FP and LSP . . . . . . . . . . . . . 115

7.4.1 Channel Hardening . . . . . . . . . . . . . 115

7.4.2 FP (Single-Sided Massive MIMO) . . . . . . . . . . 116

7.4.3 Large System Potential (Double-Sided Massive MIMO) . 116

7.5 Numerical Results . . . . . . . . . . . . . . . . . . . 118 
7.6 Conclusion . . . . . . . . . . . . . . . . . . . . . . 129

8 Conclusions and Future Work 133

8.1 Conclusions . . . . . . . . . . . . . . . . 133

8.1.1 Distributed Antenna Systems . . . . . . . . . . . . . . . 134

8.1.2 Spatial Correlation for Distributed and Centralized Sys-

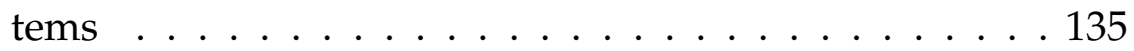

8.1.3 Asymptotic Analysis for Massive MIMO . . . . . . . . . 136

8.2 Future Work . . . . . . . . . . . . . . . . . . 138

8.2.1 Multi-user Scheduling for Massive MIMO . . . . . . . . 139

8.2.2 Multi-cell Interference Analysis for Two-stage Hybrid

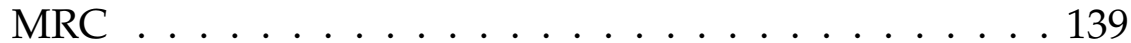

9 Appendix 141

9.1 Proof of Theorem $1 \ldots \ldots$. . . . . . . . . . . . . . . . . . .

9.2 Proof of Theorem $2 \ldots \ldots$. . . . . . . . . . . . 144

9.3 Derivation of $\mu_{\mathrm{UPA}}$ for Uniform Angular Distributions . . . . . . 148

9.4 Logarithmic growth of $\mu_{\mathrm{UPA}}$ for Uniform Angular Distributions 149 


\section{Notations and Symbols}

$|\cdot| \quad$ Magnitude of a constant and determinant of a matrix

$\|\cdot\| \quad$ Euclidean norm of a vector

$(\cdot)^{*} \quad$ Conjugate of a complex number

$(\cdot)^{-1} \quad$ Inverse of a constant or matrix

$(\cdot)^{\mathrm{T}} \quad$ Transposition of a vector or matrix

$(\cdot)^{\mathrm{H}} \quad$ Conjugate transposition of a vector or matrix

a Scalar value of $a$

a The vector a

A The matrix $\mathbf{A}$

$\mathbf{I}_{N} \quad$ An identity matrix with dimension $N \times N$

$\operatorname{diag}(\mathbf{A})$ Diagonal elements of matrix A

$\operatorname{tr}[\mathbf{A}] \quad$ The trace of the matrix $\mathbf{A}$

$\operatorname{rank}(\mathbf{A})$ The rank of the matrix $\mathbf{A}$ 
$\mathbb{E}[X] \quad$ Expectation of the random variable $X$

$\operatorname{Var}[X] \quad$ Variance of the random variable $X$

$\mathcal{C N}(\mathbf{m}, \mathbf{R})$ The circular symmetric complex Gaussian distribution with mean $\mathbf{m}$ and covariance matrix $\mathbf{R}$

$\mathrm{U}[\mathrm{a}, \mathrm{b}] \quad$ Uniform distribution on $[a, b]$

$\sim \quad$ Asymptotic equivalence

$\stackrel{\text { a.s. }}{\longrightarrow} \quad$ Almost sure convergence

$\mathcal{F}(\cdot) \quad$ Fourier transform

$J_{0}(\cdot) \quad$ The zero order Bessel function of the first kind

$I_{0}(\cdot) \quad$ The modified Bessel function of the first kind

$O(\cdot) \quad$ The growth rate of the argument

$\infty \quad$ Infinity

$\forall \quad$ For all

$\lim \{f(\cdot)\} \quad$ The limiting value of the function $f(\cdot)$ 


$\begin{array}{ll}\text { ADC } & \text { analog to digital converter } \\ \text { AoA } & \text { angle of arrival } \\ \text { AoD } & \text { angle of departure } \\ \text { BS } & \text { base station } \\ \text { CDF } & \text { cumulative distribution function } \\ \text { CSI } & \text { channel state information } \\ \text { HBF } & \text { hybrid beamforming } \\ \text { i.i.d. } & \text { independent and identically distributed } \\ \text { LoS } & \text { Line-of-Sight } \\ \text { MIMO } & \text { multiple-input-multiple-output } \\ \text { MF } & \text { matched filtering } \\ \text { MRC } & \text { maximum ratio combining } \\ \text { MMSE } & \text { minimum mean square error } \\ \text { mmWave } & \text { millimetre wave } \\ \text { MU-MIMO } & \text { multi-user multiple-input-multiple-output } \\ \text { NLoS } & \text { Non-Line-of-Sight }\end{array}$


xviii

OFDM orthogonal frequency division multiplexing

PDF probability density function

$\mathrm{RF}$ radio frequency

SE spectral efficiency

SNR signal-to-noise-ratio

SINR signal-to-noise and interference-ratio

SVD singular value decomposition

TDD time division duplexing

ULA uniform linear array

UPA uniform plannar array

$\mathrm{ZF} \quad$ zero forcing 


\section{Introduction}

A brief introduction is presented in this chapter with an overview of multipleinput-multiple-output (MIMO), multi-user MIMO (MU-MIMO), and massive MIMO techniques for future generation wireless communication systems. Then, the research motivation and problem statements are provided followed by the contributions of the thesis and a list of publications thus far.

Looking at the current vision for communication, there is an expectation of access to any information at any time and in any place, leading to an exponential demand for data transfer via wireless networks. It has been reported that the data generated in 2018 is a 6-fold increase over 2014 [1]. In order to meet the demand for an ever-increasing data rate (10 Gbps for 5G systems [2]), massive MIMO and millimetre wave are strong candidates for future commercial wireless communication systems.

Massive MIMO, scaling up the number of system antennas by at least an order of magnitude over current MU-MIMO systems, can improve the communication system's data rate, energy efficiency and link reliability [3, 4]. In addition, the desired channel properties of massive MIMO, such as favourable propagation (FP) and channel hardening, arise when operating with large arrays of antennas as a result of asymptotic random matrix theory [5]. FP means that users' channels become mutually orthogonal with an in- 
crease of the number of antennas [6,7], under which condition linear signal processing techniques such as matched-filtering (MF) and zero-forcing (ZF) benefit, resulting in near maximum transmission rates $[8,9]$. Channel hardening [10], refers to the situation where the entries of channel matrices begin to become deterministic and well conditioned, bringing the advantages of simplified precoding design and system analysis [7]. However, these idealistic channel properties are largely studied under the assumptions of an independent and identically distributed (i.i.d) channel and an infinite number of antennas. The physical size of massive MIMO arrays has been an obstacle for implementation for decades due to physical constraints. However, with the adoption of millimetre wave, where the antenna spacing is proportional to the wavelength the antenna array size becomes much smaller when operating in these high frequencies. Hence, massive MIMO is now receiving enormous research attention both in academia and industry. The channel bandwidth of $2 \mathrm{GHz}$ will become common for systems operating at above $60 \mathrm{GHz}$ [11]. Current wireless systems operate on a relatively small range of microwave frequencies which are usually from a few hundred $\mathrm{MHz}$ to a few $\mathrm{GHz}$ corresponding to wavelengths in the range of several centimetres up to a metre [2]. This precious microwave bandwidth is almost fully occupied. Even though there are some advanced signal processing techniques like cognitive radio or small cells which can unleash some spectrum, they cannot meet the required Gbits/s data rates. The main motivation for moving cellular communication systems to the millimetre wave band $(30-300 \mathrm{GHz})$ is to take advantage of the large bandwidth available at high frequencies [12], assigning users a larger communication bandwidth and enabling higher user data rates. Even though massive MU-MIMO can enable multi-user communications in the same frequency band, some beamforming techniques, e.g., maximum ratio combining, are unable to handle a large number of users due 
to their inability to mitigate interference among users. Thus, frequency reuse will need to be used alongside MU-MIMO to cut down on interference.

\subsection{Motivation}

Very large numbers of antennas are needed to obtain enough beamforming (BF) gains for next generation wireless communication systems. The large number of antennas deployed at the transmitter will cause high hardware costs, power consumption, and correlation. Some hardware components, like analog-to-digital converters (ADC), make it difficult to dedicate a separate RF chain for each antenna because they are high in power consumption and cost. A new design is required to meet this challenge and analog, digital and hybrid $\mathrm{BF}$, in which the processing is divided between the analog and digital domains, are currently being proposed. Thus, low-complexity BF techniques for massive MIMO arrays are of great importance. In particular, the simplicty of MF makes it an attractive candidate. Moreover, distributed systems provide good coverage and reduce the negative effects of correlation encountered with large antenna arrays. This motivates the central topic of the thesis, the performance analysis of MU-MIMO using low complexity MF based methods.

Within this area of research, the emergence of millimetre wave is making certain aspects of the communication channel more important. Millimetre wave channels can be highly correlated so the thesis takes a close look at the effects of correlation on MF. Also, millimetre wave channels are built on physical ray-based models. Although such models have also been used for microwave channels, ray-based models have become increasingly popular as they are essential for millimetre wave. Hence, the thesis looks at the signal power and interference power found in ray-based channels using MF. This 
relates to the basics of massive MIMO, namely FP and channel hardening. Hence, although the thesis is not focused on millimetre wave, these recent trends have guided the direction of the work. In fact, the thesis is not tied to any band although the millimetre wave band is one of the motivations for massive MIMO and hybrid beamforning (HBF). Nevertheless, ray-based models and massive MIMO and anlog processing are also being considered at microwave frequencies. The specific research problems based on this broad motivation are described below.

\subsubsection{Low-complexity Beamforming (BF) Analysis in Distributed Systems}

Due to the large antenna arrays required, one trend for future cellular systems is to separate the base station (BS), dividing the antennas into multiple antenna clusters. This is known as a collaborative BS system [13], also referred to as a distributed system, leading to a different system architecture compared with the centralized or co-located systems. Such distributed systems have the advantage of providing greater coverage than a single, co-located, antenna cluster as they both reduce the minimum and average distance to a specific user and consequently increase the average received signal-to-noiseratio (SNR) [14]. Considering hardware costs, massive MIMO systems require a simple and low power-consumption BF design. In recent years, many works have been focused on analog beamforming design and antenna selection methods. Analog beam training approaches have been developed in [15-18]. The lens-based analog BF in [19] takes advantage of beamspace expressions to achieve near-optimal digital BF performance. A novel soft antenna selection approach through RF signal processing has been proposed in [20]. In [21], an analog BF algorithm aimed at maximizing the average SNR has been developed to jointly design the transmitter and receiver analog BF 
weights. Maximal ratio combining (MRC) not only maximizes the received signal power, but also requires no central control and can be deployed independently at each antenna in a distributed BS layout [22]. Thus, there is a need to analyse the performance of analog MRC distributed systems compared with centralized conventional systems. We contribute to this analysis in Chapter 4 and Chapter 5.

\subsubsection{Spatial Correlation for Distributed and Co-located Sys- tems}

Distributed BSs with large antenna array have aroused great interest not only because they provide good coverage but also as they provide the additional benefit of reduced spatial correlation relative to large co-located arrays [22]. Since there are more antennas which are closely-spaced in one physical location, massive MIMO suffers more from spatial correlation than conventional MIMO systems [23-26]. Although distributed systems reduce this adverse impact by dividing the antennas into multiple antenna clusters, the correlation within the cluster still exists. Hence, the performance of large antenna arrays operating under correlated fading is of great interest [27-32]. While digital MU-MIMO MRC is well understood, relatively few analytical results exist for analog MU-MRC in distributed systems with correlation effects. Thus, in Chapter 4 and Chapter 5, we also focus on the impact of different correlation models on the distributed and co-located systems performance.

\subsubsection{Distributed Hybrid MRC in Two Stage MU-MIMO Sys- tems}

Distributed systems provide the benefits of reduced spatial correlation [22] and greater coverage than conventional co-located systems [13]. However, in 
a distributed system, interference among users is a major barrier to meet the required data rate. Thus, some degree of centralized processing, which coordinates the received signals from the distributed antennas [33], is required to either maximize the desired signal power or reduce the inter-user interference. The processing is done at a central node which has access to the signals received at all distributed antennas $[22,33]$. The importance and limitations of coordinated antennas are further discussed in [14,22] and references within. In fully cooperative distributed systems, where all the received signals are sent from the antenna clusters to a central processor, attractive performance gains are achieved compared to the equivalent non-cooperative network $[22,33]$. In [13], full cooperation is evaluated in the downlink with dirty paper coding and zero forcing (ZF). However, taking communication overheads and complexity into consideration, simplified processing is required. Hence, maximum ratio combining (MRC) with user grouping was proposed in [22]. To relieve the need for centralized knowledge of all the signals, twostage processing is proposed in [33]. Here, only the output signals after local (first stage) processing are sent to the (second stage) central processing node. However, there is very little work in the literature on very low complexity two-stage BF techniques for both local and central processing. Thus, in Chapter 6 we further reduce the complexity and hardware requirements of local and central processing by considering analog and digital MRC.

\subsubsection{Asymptotic Behaviour for Distributed Systems}

There are many published results concerning asymptotic analysis in centralized systems. Asymptotic capacities are derived in [7] based on digital ZF and MRC for downlink MU-MIMO where the number of users and antennas grow to infinity simultaneously but at a fixed ratio. A thorough analysis with several linear precoders and detectors in a multi-cellular massive MIMO 
system is presented for both uplink and downlink in [34]. In [27], the asymptotic channel matrix behaviour is studied with digital ZF and MRC precoders. However, there is no published work analysing the difference between analog and digital MRC with large antenna arrays in distributed systems and with different correlation models. This convergence analysis for distributed systems is discussed in Chapter 5 and Chapter 6. The massive MIMO asymptotic behaviour for ray-based channels is investigated in Chapter 7 and described in more detail below.

\subsubsection{Massive MIMO for Ray-based Channels}

The bulk of the theoretical work on FP and channel hardening has employed classical statistical channel models. However, accurate modelling of large dimensional and millimetre wave channels requires a strong link to the propagation environment, which is usually obtained through ray-based models. These models are more physically based, extensively validated by measurements, have a closer link to the array architecture and are widely used irrespective of the frequency band [35-37]. Most of the published work concerning FP and channel hardening are under specific assumptions about the underlying angular distributions, such as a uniform distribution approximation $[9,38]$. Hence, a general analysis of FP for ray-based models with arbitrary ray distributions is almost entirely lacking. In Chapter 7 , we investigate the properties of FP and channel hardening for ray-based models with the most basic assumptions concerning physical phenomena rather than statistical parameters. In addition, we extend this analysis to the asymptotic regime where both the numbers of antennas and the number of users grow to infinity at a fixed ratio (double-sided massive MIMO). Here, the equivalent question concerns whether the total interference power caused to the desired user dominates the signal power. In order to investigate this behavior, we define 
large system potential (LSP) as the ratio of the mean desired signal power to the total mean interference power.

Since the thesis considers channels which have user specific statistics and vary from i.i.d Rayleigh, through correlated Rayleigh, Ricean and ray-based, we use the term heterogeneous in the thesis title to denote the breadth of channel models considered.

\subsection{Contributions}

The contributions of this thesis, listed by chapter, are as follows.

\subsubsection{Analysis of Analog MRC for Distributed MU-MIMO Systems (Chapter 4)}

- Novel analytical expressions of expected per-user signal power and interference for both uplink MRC and downlink MF in distributed antenna systems are derived. The derivation is robust to changes in system dimension and correlation models.

- We analyse the system performance of digital and analog MRC/MF for three BS layouts. We show that while digital MRC benefits from increased BS decentralization, the same does not hold for analog MRC.

- Simulation based investigations of the impact of different Rice K factors on both centralized and distributed systems with analog and digital MRC are presented. We show that the performance of analog MRC approaches that of digital MRC when increasing the $\mathrm{K}$ factor in a centralized system, while the performance gap between the two techniques remains in a distributed system. 


\subsubsection{Analysis of Analog and Digital MRC in Massive MU- MIMO Systems over Correlated Channels (Chapter 5)}

- We derive analytical expressions of signal-to-noise and interference-ratio (SINR) for uplink analog MRC and digital MRC under different correlation scenarios when the number of antennas and users grows to infinity. The asymptotic behaviours of these cases are analysed. The findings suggest that the system performance is improved where there is perfect correlation within one user's channel but a random phase distribution among users. Thus, in some cases, correlation is beneficial and can help system performance. From the derived expression of SINR for i.i.d Rayleigh fading, around $21.5 \%$ performance loss occurs with ana$\log$ MRC compared with digital MRC. The system performance analysis under perfect correlation suggests that digital and analog MRC would have the same asymptotic behaviour.

- We derive a signal-to-interference-ratio (SIR) expression based on an exponential correlation model, which takes both correlation coefficient and phase into consideration. The 3D surface plots based on this expression give great insight into why high correlations with random phases improves system performance. We also show the similar system behaviour under a one-ring correlation model.

- The interference behaviour under perfect correlation with two special correlation models, exponential and one-ring are studied. We find that the asymptotic behaviour of interference mainly depends on the angular distribution of users. 


\subsubsection{Hybrid MRC with Imperfect CSI in Two Stage Distributed MU-MIMO Systems (Chapter 6)}

- Novel analytical expressions for the expected spectral efficiency (SE) for uplink A-D and D-D are derived.

- We analyse the system performance of A-D and D-D for two- and fourcluster BS layouts. We show that D-D benefits from increased cluster decentralization.

- Simulation results for A-ZF and D-ZF allow a performance comparison of A-D, D-D, A-ZF, and D-ZF. We show that MRC is a reasonable alternative to ZF in the second stage even at moderate SNR levels. Furthermore, A-D only requires phase shifters in the local processing and a few RF chains at central processing. Thus, A-D is a very low complexity technique for distributed systems which reduces hardware costs and power consumption.

- We show that A-D has an asymptotic performance loss of approximately $21.5 \%$ compared to D-D in centralized systems. For distributed systems, the performance comparison depends on the pattern of user link gains.

\subsubsection{Massive MIMO Asymptotics for Ray-Based Channels (Chapter 7)}

- We show that channel hardening may or may not occur depending on the nature of the model.

- We show that FP is guaranteed for all models where the ray angles are continuous random variables (as assumed by all models to date). 
- For LSP, we derive remarkably simple expressions which relate the asymptotic interference behavior to system size, antenna spacing and the ray distribution. We demonstrate that LSP will not normally hold as the interference power grows logarithmically relative to the power of the desired user channel as the system size increases.

- Analytical results are verified via simulation and using special cases for specific angular distributions where analytical results are possible.

- Despite the lack of LSP, the implications for massive MIMO are excellent. Although the interference eventually dominates the desired channel, the growth is very slow and is further attenuated by practical factors such as the likely propagation environment and the typical array patterns employed. In addition, we prove that trivial scheduling schemes can retain LSP.

\subsection{Thesis Outline}

The reminder of the thesis is organized as follows:

- Chapter 2 provides a theoretical background, including the key properties of wireless communication channel models, MIMO and MU-MIMO channel models.

- Chapter 3 examines the system architectures and beamforming techniques for massive MIMO systems. Three system architectures are discussed: analog, digital, and hybrid. Linear beamforming techniques for both single user MIMO and MU-MIMO are also included.

- Chapter 4 investigates the performance analysis for uplink analog MRC and downlink MF in a correlated Rayleigh fading channel for both centralized and distributed MU-MIMO systems. 
- Chapter 5 extends the work in Chapter 4 to asymptotic analysis with different correlation models.

- Chapter 6 proposes four low-complexity, two-stage BF techniques to improve the system performance in Chapter 4 and reduce hardware costs and power consumption.

- Chapter 7 investigates FP and channel hardening properties for raybased channels with two antenna topologies: uniform linear array (ULA) and uniform plannar array (UPA).

- Chapter 8 concludes the thesis with key contributions and future research directions.

\subsection{Publications}

- S. Li, P. J. Smith, P. A. Dmochowski and J. Yin, "Analysis of Analog and Digital MRC for Distributed and Centralized MU-MIMO Systems," IEEE Trans.Veh. Technol., pp. 1948-1952, February, 2019.

- S. Li, P. J. Smith, P. A. Dmochowski, H. Tataria, M. Matthaiou and J. Yin, "Massive MIMO for Ray-Based Channels," in Proc. IEEE Int. Conf. Commun. (ICC), July, 2019.

- S. Li, P. J. Smith, P. A. Dmochowski, H. Tataria, M. Matthaiou and J. Yin, “Massive MIMO Asymptotics for Ray-Based Propagation Channels," IEEE Trans. Wireless Commun., August, 2019, accepted.

- S. Li, P. J. Smith, P. A. Dmochowski and J. Yin, "Analysis of Analog and Digital MRC in Massive MU-MIMO Systems over Correlated Channels," IEEE, Trans. Veh. Technol., in preparation. 
- S. Li, P. J. Smith and P. A. Dmochowski, “Hybrid Distributed MRC with Imperfect CSI in MU-MIMO Systems," IEEE Wireless Commun. Lett., August, 2019, submitted. 


\section{MU-MIMO Channel Modelling}

As the performance of wireless communication is highly dependent on the channels that users experience, it is important that we have a thorough understanding of the various types of wireless communication channels and modelling methods. These methods can be categorised into two main types, statistical and ray-based. Most of the other channel models are either extended or transformed versions of these two. Firstly, this chapter presents a general overview of the propagation properties related to wireless channels, such as large-scale and small-scale fading. Then an introduction to statistical and ray-based channel models is given followed by a discussion of uplink and downlink MU-MIMO systems. Beamforming techniques, which are introduced in the following chapter, require channel information. Thus, a brief discussion of two widely used methods of attaining channel knowledge in modern wireless communication systems is given. As some of the work in the thesis looks at the asymptotic behaviour of large antenna systems, the properties of massive MIMO are also introduced. 


\subsection{The Propagation Properties of Wireless Com- munication Channels}

Unlike wired communication, where the signal is usually transmitted over wired-based media, such as copper, or optical fibre, the wireless radio channel is a much more challenging environment. Wireless channel effects include fading, interference, noise, and reflection and diffraction by obstacles. The received signal power is attenuated badly by the artefacts above and shows considerable randomness. There are three main factors that have the most important impact on radio channels and can be classified as large-scale fading (Sec. 2.1.1), small-scale fading (Sec. 2.2), and blockage and outage (Sec. 2.1.2).

\subsubsection{Distance-Based Path Loss and Shadowing}

Consider transmitting a signal between a transmitter and a receiver. A popular distance-based model for path loss is [39]

$$
P L(d)[d B]=\alpha+10 \beta \log _{10}(d)+\xi, \quad \xi \sim \mathcal{C N}\left(0, \sigma_{\mathrm{SF}}^{2}\right)
$$

where $P L(d)$ is the path loss of a link at distance $d$ and $\xi$ is shadowing. Depending on the scenario, some values of parameters $\alpha$ and $\beta$ can be found in [39]. When $\beta=2$ and there is no uncertainty, $\xi=0,(2.1)$ can be converted to the free-space Friis' law [40]

$$
P=G_{r} G_{t}\left(\frac{\lambda}{4 \pi d}\right)^{2} P_{t}
$$

where $P$ is the received power, $P_{t}$ is the transmit power, $G_{t}$ and $G_{r}$ are the transmit and the receive antenna gains respectively, and $\lambda$ is the wavelength. As we know, $\lambda=\frac{v}{f}$, where $v$ is the speed of light and $f$ is the carrier frequency. 
Thus, the received power, $P$, has an inverse square law decay with $f$. At high frequencies, the much smaller wavelength not only means high path loss, but it also means that more antennas can be closely spaced into the same physical area [11], which creates possibility BF and antenna gains to counteract the large path loss. Thus, large array systems which are equipped with large directional antenna arrays can have reasonable coverage. The measurements conducted in New York City [41,42] have inspiring results to support this.

Due to obstacles, which cause scattering, absorption, diffraction and reflection, the received signal power experiences a great deal of randomness. All the factors above introduce a random component into the path loss (see (2.1.1)), which is often modelled as a log-normal random variable. In particular,

$$
\varphi=10^{(\xi / 10)},
$$

where $\xi$ is the zero mean Gaussian variable in (2.1) and $\sigma_{\mathrm{SF}}$ is the shadowing standard deviation in $\mathrm{dB}$.

\subsubsection{Blockage and Outage}

The theoretical high path loss over millimetre wave bands can be partly compensated for by directional antennas. Blockage is another big challenge which can attenuate signals by as much as $30 \mathrm{~dB}$ to $80 \mathrm{~dB}$ depending on different blocking materials [41,42]. For high frequency, diffraction is less significant while LoS is more significant, making blockages more important. However, small wavelengths due to high frequency increase the effective roughness of materials, which results in more scattering, an important mechanism in enabling coverage via NLOS paths. Several models can be used to evaluate the effect of blocking, like a two-state model considering the scenarios: line of sight (LOS) and NLOS, and a three-state model including LOS, NLOS, and signal outage [41,42]. Such models can help to analyse the coverage and ca- 
pacity in large antenna array systems.

\subsection{Statistical Channel Models}

Due to the complexity of the small scale fading phenomenon in wireless channels it can be difficult to describe wireless links via simple, physically based models. Hence, statistical channel models are often used which represents a simplified view of the actual environment. In this section, we introduce two widely used statistical channel models, i.i.d. Rayleigh and i.i.d. Ricean, which are also the building blocks of many more complicated channel models.

\subsubsection{I.I.D Rayleigh}

The i.i.d Rayleigh model is commonly used in urban areas, where rich scattered replicas of the transmitted signals arrive at the receiver and no direct path is assumed. Here, the normalized received signal's amplitude has the probability distribution function (PDF) given by $[40,43]$

$$
f_{R}(r)=2 r e^{-r^{2}}, r \geq 0,
$$

and the phase angle has a uniform distribution from 0 to $2 \pi$. The simplicity of this model allows closed-form analysis in many applications.

\subsubsection{I.I.D Ricean}

We employ a Ricean channel model $[40,43]$ when there is rich scattering, as in the Rayleigh model, but also a line-of-sight (LoS) path between transmitter and receiver. The channel is described by two parameters: $K$ and $\Omega$, where $K$ is the ratio of the deterministic power to the scattered components and $\Omega$ represents the total power of the two kinds. The amplitude of the received 
signal has the PDF

$$
f_{R}(r)=\frac{2(K+1) r}{\Omega} \exp \left(-K-\frac{(K+1) r^{2}}{\Omega}\right) I_{0}\left(2 \sqrt{\frac{K(K+1)}{\Omega}} r\right), r \geq 0
$$

where $I_{0}$ is the zeroth order modified Bessel function of the first kind.

\subsection{Narrowband MIMO Channel}

In this section, we discuss both single-user (also known as point-to-point) MIMO and multi-user narrow band MIMO channels. MIMO exploits and takes advantage of diverse channels between the transmitter and the receiver, where both sides are equipped with multiple antennas [44]. The independent channels that MIMO systems can use gives rise to multiplexing gain, which can improve system performance massively and has been already deployed in current wireless communication systems [45].

\subsubsection{Narrowband Single-user MIMO Channel}

Single-user MIMO refers to the system where there are $N_{t}$ transmit antennas at the BS, serving only one user equipped with $N_{r}$ antennas. This system can be simply represented as [40]

$$
\mathbf{y}=\mathbf{H x}+\mathbf{n},
$$

where $\mathbf{H}=\left[\begin{array}{rrr}h_{11} & \cdots & h_{1 N_{t}} \\ \vdots & \ddots & \vdots \\ h_{N_{r} 1} & \cdots & h_{N_{r} N_{t}}\end{array}\right]$ is the $N_{r} \times N_{t}$ channel matrix and $h_{i j}$ represents the channel gain from transmit antenna $j$ to receive antenna $i$. These channels can have various distributions, such as i.i.d Rayleigh in Sec. 2.2.1, i.i.d Ricean in Sec. 2.2.2 or more complex distributions. In (2.6), $\mathrm{x}$ is the trans- 
mitted symbol vector $\left(N_{t} \times 1\right)$, $\mathbf{y}$ is the received vector $\left(N_{r} \times 1\right)$ and $\mathbf{n}$ is the $N_{r} \times 1$ noise vector.

\subsubsection{Narrowband Multi-user MIMO Channel}

Multi-user MIMO (also know as MU-MIMO) systems use the same time and frequency resource to serve multiple users simultaneously. This technique not only improves system performance with limited resources, but also can adopt simple signal processing techniques such as MF with growing numbers of antennas [46]. The users served can be equipped with multiple antennas, as described in Sec. 2.3.1, or just with one single antenna. In our work, we only consider MU-MIMO with a single antenna at the user equipment (UE).

\section{Uplink Multi-user Channel}

Now consider uplink transmission for a MU-MIMO system as shown in Fig. 2.1. The base station is in the middle of a cell, and users are distributed within the cell. Hence, the uplink multi-user global channel is

$$
\mathbf{H}=\left[\mathbf{H}_{1} \mathbf{H}_{2} \cdots \mathbf{H}_{K}\right]
$$

where $K$ is the total number of users and $\mathbf{H}_{i}$ is the channel for user $i$.

\section{Downlink Multi-user Channel}

Downlink transmission for a multi-user system is as shown in Fig 2.2. Similarly, the downlink multi-user global channel is

$$
\mathbf{H}=\left[\mathbf{H}_{1}^{\mathrm{H}} \mathbf{H}_{2}^{\mathrm{H}} \cdots \mathbf{H}_{K}^{\mathrm{H}}\right]^{\mathrm{H}},
$$

where $K$ is the total number of users and $\mathbf{H}_{i}$ is the channel for user $i$. . 


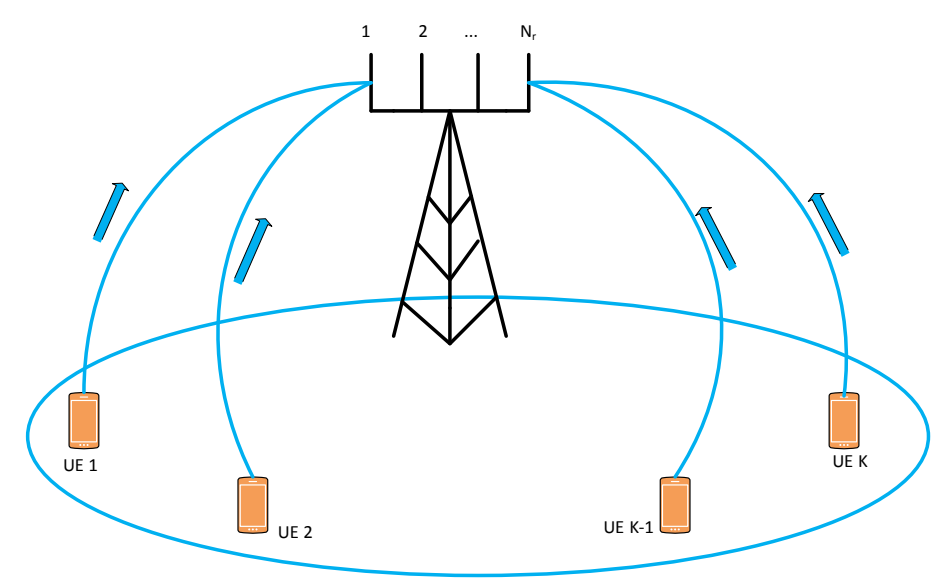

Figure 2.1: Uplink multi-user channel

\subsection{Ray-based MIMO Channel Models}

In order to model diffraction and reflection effects, ray-based models have been used for decades in mobile terrestrial channel [47]. Due to the physicalbased nature and close link to the array architecture, ray-based models are widely used for system analysis [35-37]. Especially when the wavelength is relatively small, the total channel behaves like the sum of narrow beams or rays [47]. Thus ray-based channel models are vital for next generation wireless communication systems as high frequencies are more likely to be used such as millimetre wave, whose wavelength is relatively small, leading 


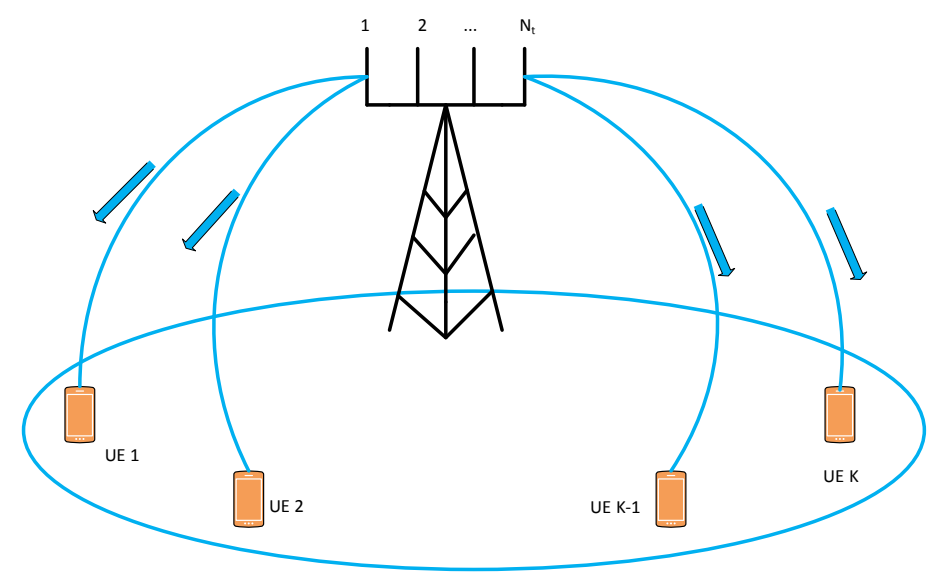

Figure 2.2: Downlink multi-user channel

to different propagation effects and possibly a smaller number of rays. The typical parameters of low frequency systems, such as angle spread, time delay spread, etc., will have different values in millimetre wave environments. Many measurements [39] show that the millimetre wave channel tends to have only a few clustered paths which are sparse in the angular domain [11]. Many other popular channel models, such as the 3GPP standardization exercise [35] and Saleh-Valenzulela (SV) type [48] channel models are variations of such ray-based models. The ray-based MIMO channel can be described either by a $2 \mathrm{D}$ or a $3 \mathrm{D}$ multipath channel model. For a $2 \mathrm{D}$ channel model, the antenna array steering vectors only consider azimuth angles; whereas both az- 
imuth and elevation angles will be used for a 3D channel model. We assume the channel is narrowband block-fading. Note that our work can be applied to both microwave and millimetre wave systems. The narrowband assumption based on the use of orthogonal frequency-division multiplexing (OFDM) is a well-accepted assumption in microwave systems. However, in higher frequency bands, this assumption is controversial due to the high peak-toaverage power ratio (PAPR) problem with OFDM compared to single-carrier systems $[49,50]$. [51] shows that single-carrier with minimum mean-squared error (MMSE) performs close to OFDM, but with a lower PAPR. Also, in [49], it is shown that OFDM is similar to single-carrier if amplifier non-linearities can be handled, but worse than single-carrier otherwise. Thus, both OFDM and single-carrier techniques remain candidates for higher frequency bands. However, OFDM remains the chosen technique for future generation systems in the 3 GPP standards ${ }^{1}$. Hence, the channel for a single antenna UE can be represented by [42]

$$
\mathbf{h}=\frac{1}{C L} \sum_{c=1}^{C} \sum_{l=1}^{L} g_{c l} \mathbf{a}\left(\phi_{c l}\right)
$$

where $C$ is the number of clusters; $L$ is the number of subpaths in each cluster; if only considering azimuth domain, each subpath contains azimuth angle $\phi_{c l}$, and $\phi_{c l}=\phi_{c}$ (cluster central angle) $+\Delta$ (subarray offest angle); $g_{c l}$ is the complex small-scale fading gain on the $l$ th subpath of the $c$ th cluster. The raybased channel in azimuth domain is shown in Fig. 2.3. The elevation angle is shown in Fig. 2.4. This model is applied in Chapter 7 using the simplified notation

$$
\mathbf{h}_{i}=\sum_{r=1}^{P} \gamma_{i r} \mathbf{a}\left(\phi_{i r}\right),
$$

where $i$ is the user number, $P$ is the total number of paths $(P=C L), \gamma_{i r}$ corresponds to some $g_{c l} /(C L)$ and $\phi_{i r}$ is one of the $\phi_{c l}$ angles. 3D channel

\footnotetext{
13GPP TR 21.915 V15.0.0, http://www.3gpp.org/.
} 
models also include elevation angles which are defined in terms of clusters in a similar manner to Fig. 2.3. This is described in more detail in Chapter 7.

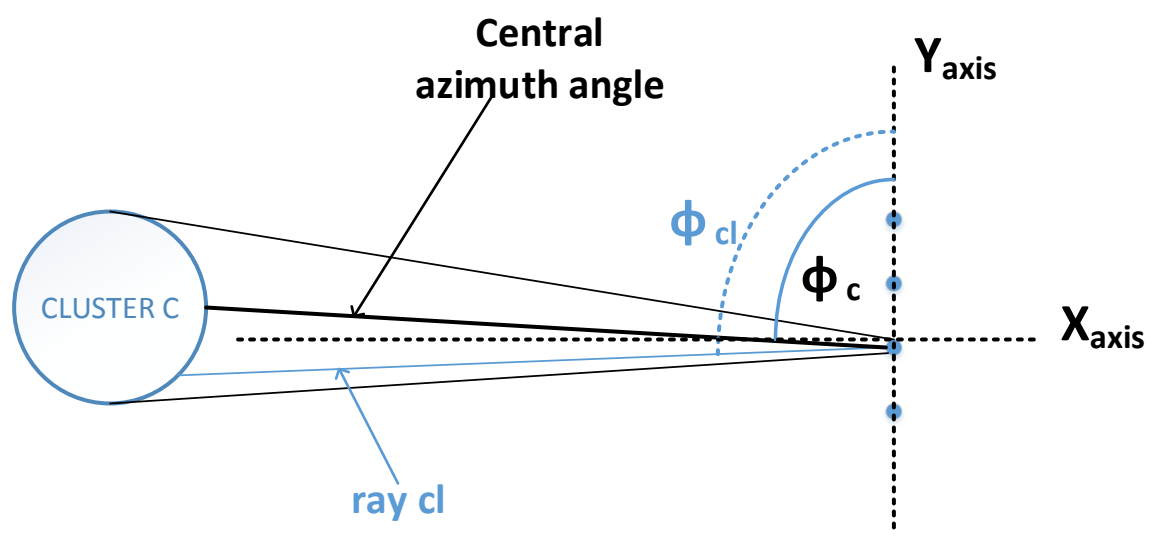

Figure 2.3: Ray-based channel model showing a generic cluster in the azimuth domain with the azimuth central angle of the cluster and the offset of a given ray.

\subsection{Spatial Correlation}

MIMO techniques exploit the spatial aspect of communication channels [47]. Spatial correlation of two or more channels may occur in such a structure, which in turn may have an impact on MIMO system performance. Thus, to characterize the spatial properties of a MIMO system it is important to consider the channel correlation structure. Here, we discuss spatial correlation at the transmitter, but this can also be applied to the receiver. We define the 


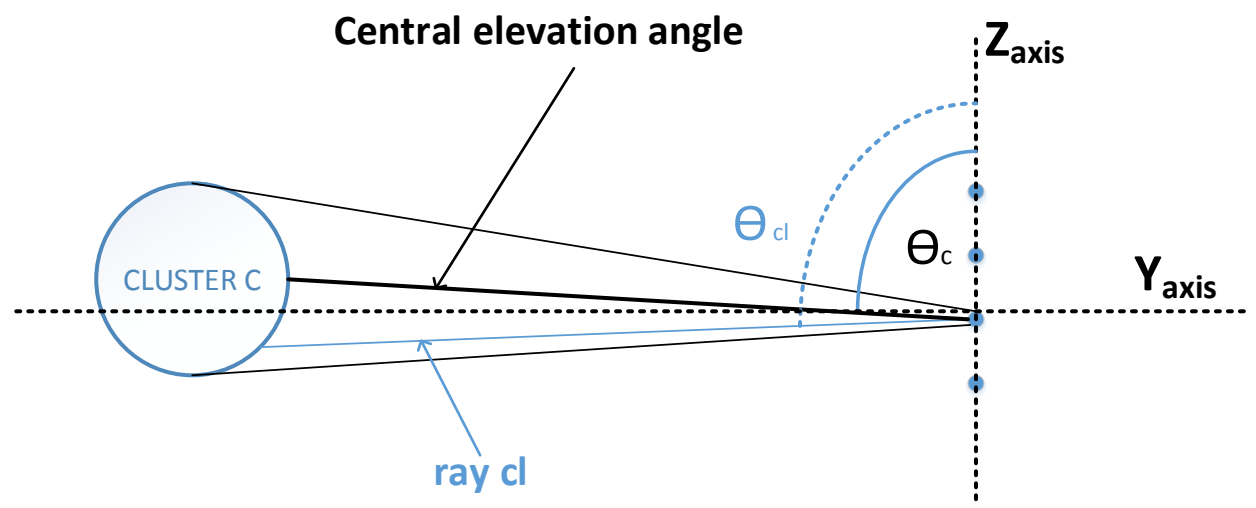

Figure 2.4: Ray-based channel model showing a generic cluster in the elevation domain with the elevation central angle of the cluster and the offset of a given ray.

spatial correlation coefficient between two transmit antenna elements $q$ and $q^{\prime}$ as $R_{q, q^{\prime}}$, which satisfies $0 \leqslant\left|R_{q, q^{\prime}}\right| \leqslant 1$. If $\left|R_{q, q^{\prime}}\right|=1$, we say that the two antenna element channels are fully correlated, while $R_{q, q^{\prime}}=0$ implies that the channels are uncorrelated $[47,52]$. Many factors, such as the numbers of clusters and subpaths, angular spread in (2.9) and various antenna topologies will change the level of correlation. We give two widely-used correlation models, Exponential and One-ring in Sec. (2.5.1) and Sec. (2.5.2), separately. 


\subsubsection{Exponential}

The exponential spatial correlation model is the simplest, common model for correlation, widely adopted as in $[53,54]$. This model is ideal for analysis due to its use of a single parameter to characterize the level of correlation. The exponential correlation matrix at the BS (similarities applies to the receiver) can be described by

$$
\mathbf{R}=\left[\begin{array}{rrrr}
1 & \rho & \cdots & \rho^{N_{t}-1} \\
\rho^{*} & 1 & \cdots & \rho^{N_{t}-2} \\
\vdots & \vdots & \ddots & \vdots \\
\left(\rho^{N_{t}-1}\right)^{*} & \left(\rho^{N_{t}-2}\right)^{*} & \cdots & 1
\end{array}\right]
$$

where $\rho$ is the decay parameter and $|\rho| \leqslant 1$. The correlation effect decays exponentially as the distance of two elements increases. Hence, it is best applied to linear arrays. Large $|\rho|$ indicates a high correlation effect.

\subsubsection{One-ring}

The one-ring correlation model is proposed based on the characteristics of the physical environment [55-60]. This type of correlation matrix captures the differences in the dominant propagation channel to each terminal, which are mainly characterized by two physical quantities: (1) the local scattering environment and (2) the antenna spacing distance at the transmit array [43, $61,62]$. The coefficients of a one-ring correlation matrix for a uniform linear array (ULA) array can be described [63] as

$$
[\mathbf{R}]_{i, j}=\frac{1}{2 \pi} \int_{0}^{2 \pi} e^{j 2 \pi d_{s}(i, j) \sin (\theta)} d \theta
$$

where $d_{s}(i, j)$ is the distance between antenna $i$ and antenna $j$. 


\subsubsection{Correlated MIMO Channel}

The principle of modelling a correlated MIMO channel is usually to apply the correlation matrix such as those in Sec. 2.5.1 or in Sec. 2.5.2 at either the BS or the receiver or both sides. The channel can be described via the Kronecker product model as $\hat{\mathbf{H}}=\mathbf{R}_{\mathrm{R}}^{1 / 2} \mathbf{H}_{i . . . d} \mathbf{R}_{\mathrm{T}}^{1 / 2}$ where $\mathbf{R}_{\mathrm{T}}\left(N_{t} \times N_{t}\right)$ describes the correlation effect among the transmit antennas and $\mathbf{R}_{\mathrm{R}}\left(N_{r} \times N_{r}\right)$ is the correlation matrix at the receiver $[8,57]$. The matrix, $\mathbf{H}_{\text {i.i.d, }}$, is an i.i.d channel matrix. Note that this is usually only valid for generating correlated Rayleigh channels.

\subsection{Channel State Information (CSI)}

MIMO is well-known for its advantages in increasing data rates by multiplexing or improving system performance by diversity techniques [40]. Most of these gains require channel knowledge at either the receiver or the transmitter or both. There are two typical methods that can provide CSI at the transmitter, which are feedback and channel reciprocity [64]. In this work, we assume a time-division duplex (TDD) system, and hence the transmitter knows the CSI via reciprocity as described in Sec. 2.6.1. Note that arrays with large numbers of antennas can be deployed in both TDD and frequency-division duplexing (FDD) systems, although there are problems with both systems. The effectiveness of the TDD system relies on the assumption that the channel coherence time is relatively long (compared with the duration of the data frame), which is proportional to $\frac{\text { wavelength }}{\text { speed }}$. Thus, for a high frequency, high speed scenario, this assumption may become invalid. In [65], with a carrier frequency of $3.7 \mathrm{GHz}$, and a bandwidth TDD of $20 \mathrm{MHz}$, acceptable prototype results were obtained up to a maximum speed of $29 \mathrm{~km} / \mathrm{h}$. As the frequency is increased further, it may be that lower maximum speeds are required. Although FDD avoids this problem, the heavy overhead of CSI acquisition in massive 
MIMO systems makes FDD undesirable. Many published works for massive MIMO still assume a TDD system [66] (and references within).

\subsubsection{Feedback}

Feedback is an approach to obtain CSI at the TX in which the receiver estimates the channel via pilots and communicates the channel back to the TX [64]. Only the essential information is transmitted as the bandwidth of the feedback link is often limited. However, for millimetre wave system with large antenna arrays, the overhead may be too much as feedback grows with the number of antennas [67].

\subsubsection{Reciprocity}

In TDD single-frequency systems, downlink and uplink channels use the same carrier frequency for transmission. The channel for the uplink is the transpose of the channel for the downlink. The transmitter can measure CSI directly without the need of feedback from the receivers [67]. Note that the 3GPP LTE TDD mode has been used in cellular systems [67].

\subsection{Massive MIMO Properties}

MIMO techniques are widely used in current wireless communication systems like WLAN (IEEE 802.11n/ac) and cellular systems (3GPP LTE and LTE Advanced) [11] which operate at microwave frequencies with only a small number of antennas (up to eight, but two is most common). In recent years, it has become commonly accepted in information theory $[68,69]$, industry $[70,71]$ and international standards $[72,73]$ that large systems, also known as massive MIMO, are a key enabler for high data rate wireless communication. Such systems communicate with many users simultaneously us- 
ing large numbers of antennas $[73,74]$, achieving the benefits of high data rates, high power efficiency and reliability with linear signal processing techniques [38]. The number of transmit antennas and receive antennas are significantly scaled up by at least an order of magnitude in a massive MIMO system compared with the conventional MIMO systems that are currently being used. Note that all the theoretical properties that conventional MIMO systems have, also apply to the massive MIMO systems. However, there are many benefits only arising from massive MIMO systems. The mathematical principles behind the large system benefits can be found using random matrix theory. Two key principles behind the success of massive MIMO are favourable propagation (FP) [3,38], and channel hardening [75]. For the following description, $\mathbf{h}_{i}$ is the channel vector for user $i$ which has a single antenna.

- Favourable Propagation

FP relates to the orthogonality of $\mathbf{h}_{i}$ and $\mathbf{h}_{j}$ and was originally defined in three flavours [38]: exact FP where $\mathbf{h}_{i}^{\mathrm{H}} \mathbf{h}_{j}=0$; approximate FP where $\mathbf{h}_{i}^{\mathrm{H}} \mathbf{h}_{j} \approx 0$ and asymptotic FP where $\mathbf{h}_{i}^{\mathrm{H}} \mathbf{h}_{j} / N \rightarrow 0$ as $N \rightarrow \infty$. Since the majority of the literature and the important properties of FP concern asymptotic FP, for simplicity we refer to asymptotic favourable propagation as FP. Here, FP denotes asymptotic FP where $\mathbf{h}_{i}^{\mathrm{H}} \mathbf{h}_{j} / N \stackrel{\text { a.s. }}{\rightarrow} 0$ as $N \rightarrow \infty$ [38]. When the number of the BS antennas becomes large, FP occurs when users' channels become orthogonal [6]. This enables simple, linear processing techniques, such as MF and ZF, to maximize the system capacity.

\section{- Channel Hardening}

Channel hardening refers to the property that the entries of the elements of $\frac{\mathbf{h}_{i}^{\mathrm{H}} \mathbf{h}_{i}}{N_{\mathrm{t}}}$ becomes deterministic as $N_{\mathrm{t}} \rightarrow \infty$ [75]. This leads to simplified system analysis and precoder design since easy matrix computation be- 
comes available [46].

Furthermore, millimetre wave MIMO envisages the use of 32 to 256 antennas. These large antenna arrays have three advantages, especially for millimetre wave systems: (1) they provide BF and antenna gains to overcome large pathloss; (2) they establish reliable links; (3) they improve spectral efficiency and can approach system capacity [76]. 


\section{System Architectures and Beamforming Techniques For Massive MU-MIMO Wireless Communications}

In order to meet the demand for an ever-increasing data rate (10 Gbps for 5G systems [2]), millimetre wave has become a strong candidate for future commercial wireless communication systems. It is envisaged that channel bandwidth of $2 \mathrm{GHz}$ will become common for systems operating at above 60 $\mathrm{GHz}$ [11]. The current wireless systems operate on a relatively small range of microwave frequencies which are usually from a few hundred $\mathrm{MHz}$ to some $\mathrm{GHz}$ with wavelengths in the range of several centimetres up to a metre [2]. This precious microwave bandwidth is almost fully occupied. Even though there are advanced signal processing techniques like cognitive radio or small cells which can unleash some spectrum, they cannot meet the required Gbps data rates. The main motivation for moving cellular communication systems to the millimetre wave band $(30-300 \mathrm{GHz})$ is to take advantage of the large bandwidth available at high frequencies [12], assigning users a larger communication bandwidth thus enabling higher user data rates. Large antenna arrays, however, result in large precoding and receive combining matrices, which increase system complexity, and also boost the training overhead for 
channel estimation. Furthermore, the large number of antennas deployed at the transceiver will cause high hardware costs and power consumption. Some signal components, like analog-to-digital converters (ADCs), make it difficult to dedicate a separate RF chain for each antenna because of their high power consumption and hardware costs. Thus, three system architecture candidates for massive MIMO have been proposed as in [67] to explore the trade-off between system performance and cost. Fig. 3.1, Fig. 3.2, and Fig. 3.3 show these three important system architectures with a 1-D planar array and the corresponding BF strategies are shown in Fig. 3.4. $N_{\mathrm{BS}}$ is the total number of antennas at the BS. $N_{\mathrm{s}}$ is the number of data streams, and $N_{\mathrm{RF}}$ is the number of RF chains..

\subsection{Analog BF Architecture}

Due to low costs in both hardware and power consumption, the purely analog architecture forms an attractive design option. Only one single RF chain with $N_{\text {BS }}$ phase shifters is required in analog BF systems. As shown in Fig. 3.1, for a downlink system with $N_{\mathrm{s}}=1, N_{\mathrm{RF}}=1, N_{\mathrm{BS}}=4$, the signal $\mathrm{s}_{1}$ passes through the only RF chain and then splits into four sub-branches. These four sub-branches adjust the phases, then transmit the signal $s_{1}$ with the same amplitude, given by $\left(\mathrm{F}_{\mathbf{B B}}\right)_{11}$, but four different phases, given by $\left(\mathrm{F}_{\mathbf{R F}}\right)_{11},\left(\mathrm{~F}_{\mathbf{R F}}\right)_{21}$, $\left(F_{R F}\right)_{31}$, and $\left(F_{R F}\right)_{41}$, on four different antennas.

Analog BF, employs a network of phase shifters to control and steer the phase of the signal [77]. Due to the large number of antennas deployed at the transmitter, the BF gains due to analog BF can be greatly increased, and the beamwidth can be reduced, which has a positive effect on interference mitigation [78]. However, the hardware constraints of analog BF, such as the use of phase shifters with no ability to change signal amplitudes, means that ana- 
$\log$ BF has a low performance compared with full digital BF [77,79]. Today's indoor millimetre wave systems, which are usually short range and time invariant, adopt the analog BF method, including three key techniques: a sector level sweep to determine the best sector, beam refinement for sharpening, and beam tracking [12]. Beam training approaches for analog BF, without CSI assumption, have been developed in [15-18]. This method is based on a closed-loop beam training process which starts at a wide beam and then reduces the beamwidth until reaching a desired resolution without channel knowledge [80].

Due to analog processing simplicity and low-cost, such architectures have received great attention for massive MIMO and millimetre wave systems. There have been many recent developments in analog architecture. The use of low-resolution ADC in lens-based methods and its impact are investigated in [81]. A novel soft antenna selection approach through RF signal processing has been proposed in [20]. The indoor $60 \mathrm{GHz}$ scenario has been considered in [21, 82]. Sub-array architectures have been investigated in [83-87] for outdoor scenarios.

\subsection{Digital BF Architecture}

The digital BF approach requires a total number of $N_{\mathrm{BS}}$ antenna RF chains and the per-element BF weights are applied digitally. As shown in Fig. 3.2, for a downlink system with $N_{\mathrm{s}}=1, N_{\mathrm{RF}}=2, N_{\mathrm{BS}}=2$, signal s 1 passes through two RF chains and then is split into two sub-branches separately for each RF chain.

Digital BF can achieve advanced transmission strategies and provide high flexibility but at the expense of power, cost, and high system complexity - a particularly important concern for massive MIMO and next generation wire- 


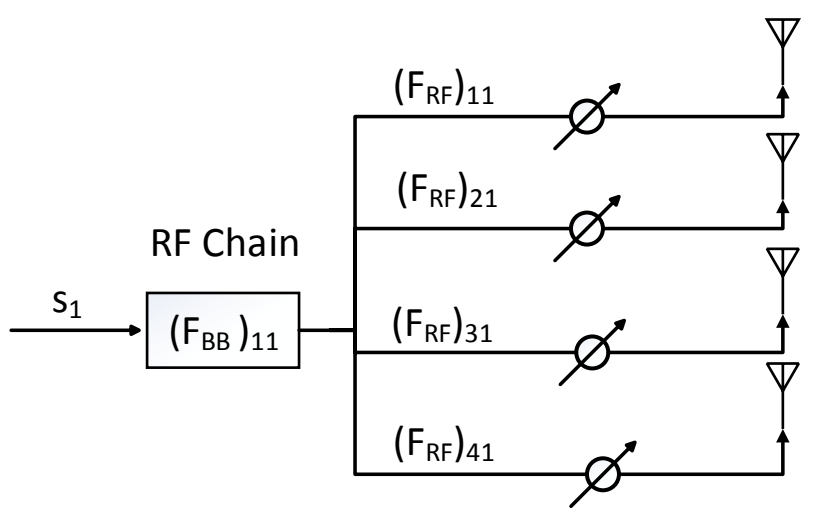

Figure 3.1: Analog system architecture, $N_{\mathrm{s}}=1, N_{\mathrm{RF}}=1, N_{\mathrm{BS}}=4$.

less communication systems. Conventional digital signal processing in the baseband is a suitable approach for low frequencies, but when it comes to high carrier frequencies with larger numbers of antennas, there are many hardware constraints. Hardware costs, space limitations, power consumption, and the complexity of large antenna arrays prevent the use of a separate RF chain per antenna [11].

Analog MRC is basically digital equal gain combining. For analog MRC, the differences in the weights across the antennas is controlled only by phase shifters, giving the weight vector, $w_{\mathrm{AMRC}}=\left[e^{-j \phi_{1}}, e^{-j \phi_{2}}, \cdots, e^{-j \phi_{N_{\mathrm{BS}}}}\right]$, which cophases the signals on each branch to match the channel and then combines them with equal weighting. This is the same as digital equal gain combining as described [40, p. 212]. Due to analog MRC's inability to match the ampli- 
tude of the signals (having only one RF chain), there is a performance loss compared with digital MRC.

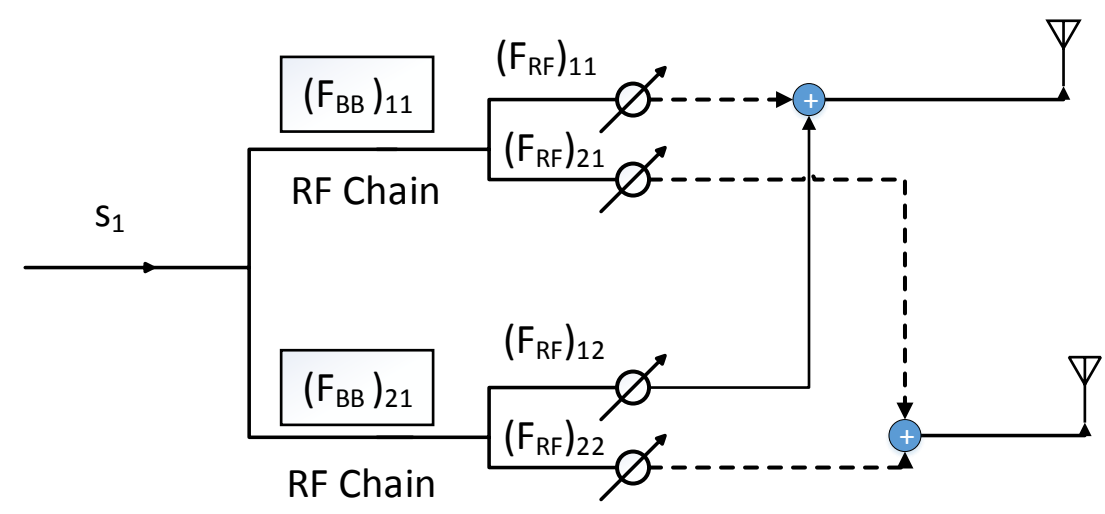

Figure 3.2: Digital system architecture, $N_{\mathrm{s}}=1, N_{\mathrm{RF}}=2, N_{\mathrm{BS}}=2$.

\subsection{Hybrid Analog-Digital Precoding Architecture}

Hybrid precoding architecture offers a compromise between digital BF and analog BF. The additional digital layer provides more freedom compared to analog BF. Compared to the full digital design, the performance of the hybrid architecture is lower but it requires fewer RF chains, and at the same time provides more flexibility than the full analog design [12]. As shown in Fig. 3.3, for a downlink system with $N_{\mathrm{s}}=1, N_{\mathrm{RF}}=2, N_{\mathrm{BS}}=4$, signal $\mathrm{s}_{1}$ is transmitted by two RF chains and then split into four sub-branches separately for each 
RF chain. In [88], the relationship between the number of RF chains and the number of data streams, is investigated and it is shown that if the number of $\mathrm{RF}$ chains is twice or greater than the number of data streams, hybrid BF can achieve full digital BF performance.

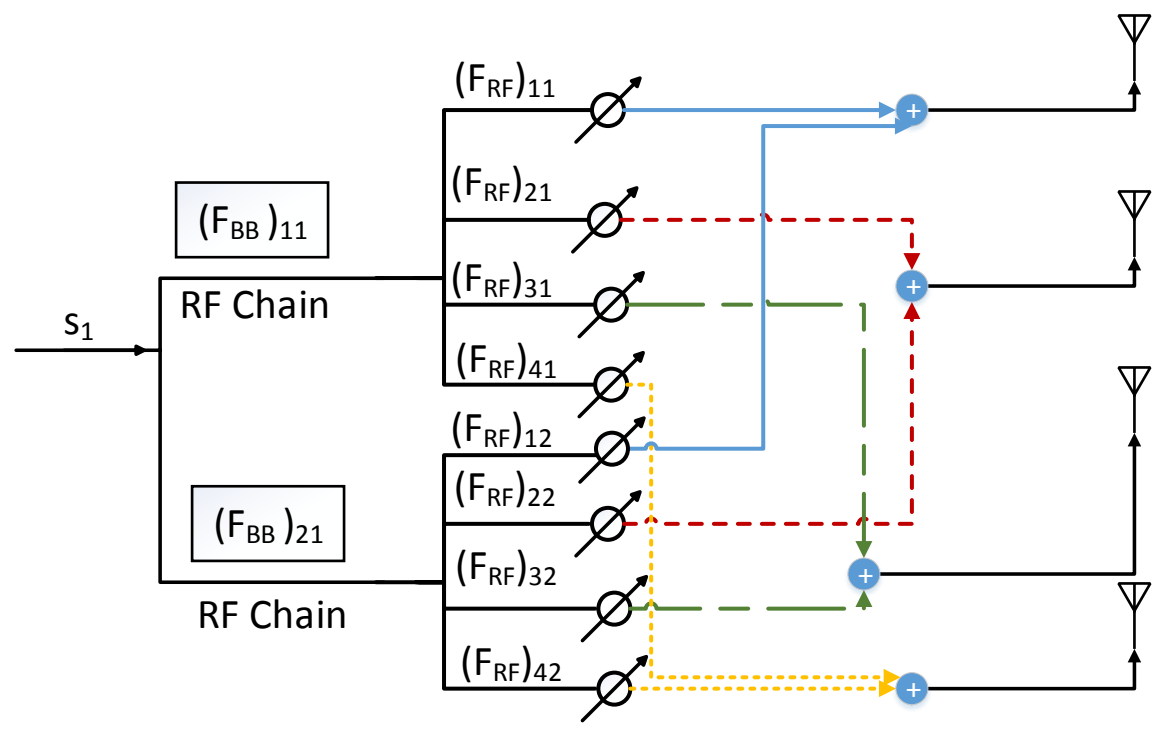

Figure 3.3: Hybrid system architecture, $N_{\mathrm{s}}=1, N_{\mathrm{RF}}=2, N_{\mathrm{BS}}=4$.

\subsection{The Relationship between Analog, Digital, and Hybrid BF}

Figure 3.4 shows the downlink (uplink is similar) precoding strategies under the three different system architectures. The general idea of precoding is to apply a linear processing matrix $\mathbf{F}$ to the transmitted signal vector. For 
the analog architecture, which is implemented in the RF domain using phase shifters, $\mathbf{F}=\mathbf{F}_{\mathrm{RF}}\left(N_{\mathbf{B S}} \times N_{\mathrm{RF}}\right)$. For the digital architecture, which is implemented in baseband, $\mathbf{F}=\mathbf{F}_{\mathrm{BB}}\left(N_{\mathrm{RF}} \times N_{s}\right)$. For the hybrid architecture, $\mathbf{F}=$ $\mathbf{F}_{\mathrm{RF}} \mathbf{F}_{\mathrm{BB}}\left(N_{\mathrm{BS}} \times N_{s}\right)$, is implemented in both RF and baseband domains.

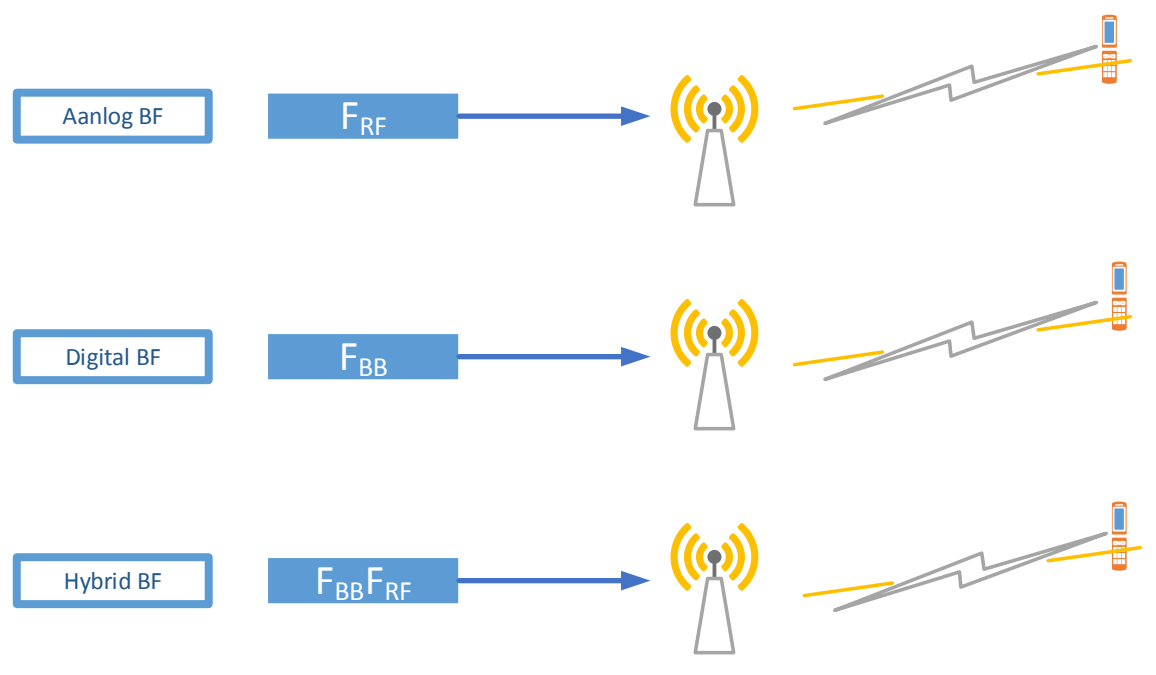

Figure 3.4: Precoding strategies under three system architectures

Our research work looks at the three BF strategies: pure analog MRC, pure digital MRC, and hybrid analog (or digital) MRC and digital ZF. The performance analysis of analog and digital MRC in centralized and distributed systems is given in Chapter 4 and its extended correlation and asymptotic analysis is presented in Chapter 5. Two-stage hybrid BF is analysed in Chapter 6. 


\subsection{Linear Beamforming}

The computational complexity of baseband processing will become impractical when a large number of antennas are deployed in a massive MIMO system. Non-linear signal processing techniques, such as dirty-paper coding (DPC) [89] and vector perturbation [90,91], which achieve better performance than linear processing, also become too difficult for practical implementation. Thus, linear precoding techniques for a downlink system, zero-forcing $(\mathrm{ZF}), \mathrm{MF}$, block diagonalization (BD) and signal to leakage and noise ratio (SLNR) [92], have received significant attention, especially with various complexity reduction techniques. There are many measurements which show that ZF can achieve a high percentage of DPC performance even with a relatively small antenna array [28,93]. Also, as discussed in Sec. 2.7, linear processing techniques of $\mathrm{ZF}$ and $\mathrm{MF}$, can achieve satisfying system performance when equipped with a large number of antennas. Similarly, simple, low-complexity combining techniques for an uplink system, such as MRC and ZF, have the same advantages as in downlink systems. There are two main categories of beamforming techniques available when we refer to MIMO systems: singleuser and multi-user, which are introduced in Sec. 3.5.1 and Sec. 3.5.2, respectively.

\subsubsection{Single-user MIMO Beamforming}

For the single-user case, in which no inter-user interference is present, the optimal linear digital precoding is based on the well-known singular value decomposition (SVD) of the channel. Any MIMO channel matrix, $\mathbf{H}$, of dimension $N_{r} \times N_{t}$ can be represented as

$$
\mathbf{H}=\mathbf{U D}^{\frac{1}{2}} \mathbf{V}^{\mathrm{H}}
$$


where $N_{r}$ and $N_{t}$ are the number of the receiver antennas and transmitter antennas, respectively. The matrix $\mathbf{U}$, of dimension $N_{r} \times N_{r}$, and the matrix $\mathbf{V}$, of dimension $N_{t} \times N_{t}$, are unitary; $\mathbf{D}^{\frac{1}{2}}$ is a $N_{r} \times N_{t}$ diagonal matrix with nonnegative diagonal entries. The columns of $\mathbf{V}$ are the eigenvectors of $\mathbf{H}^{\mathrm{H}} \mathbf{H}$ and the columns of $\mathbf{U}$ are the eigenvectors of $\mathbf{H} \mathbf{H}^{\mathrm{H}}$. When the transmitter has CSI and adopts water-filling power allocation, the optimal linear digital precoder is $\mathbf{V} \mathbf{D}_{w f}\left(\mathbf{D}_{w f}\right.$ is the square root of a diagonal power allocation matrix) and the optimal receiver combiner is $\mathbf{U}^{\mathrm{H}}[45,94]$.

\subsubsection{Multi-user MIMO Beamforming}

MU-MIMO is a key technique for next generation wireless communication systems due to its ability to serve many users in the same time and frequency resource. However, serving many users simultaneously results in interference. Hence, beamforming techniques, which can either maximize the desired user's signal power (such as MRC or MF) or mitigate users' interference (such as $\mathrm{ZF}$ ) are of high interest. Thus, two main BF techniques, MRC/MF and $\mathrm{ZF}$, are considered for massive MIMO systems.

\section{MRC}

MRC aims to maximize the desired received signal power by multiplying the received signal with the Hermitian transpose of the channel matrix, $\mathbf{H}^{\mathrm{H}}$. From the geometric perspective, $\mathrm{MRC}$, in fact, projects the received signal on to a direction which maximizes the projection length of the signal. Note that independent Gaussian noise has the same projection length in any direction, a property of isotropic noise, thus MRC only needs to maximize the projection length of the signal. In other words, MRC aims to maximize SNR. 
$\mathbf{Z F}$

$\mathrm{ZF}$, in contrast to $\mathrm{MRC}$, aims to minimize the interference by projecting the signal on to the direction orthogonal to the interference by multiplying the received signal by $\left(\mathbf{H H}^{\mathrm{H}}\right)^{-1} \mathbf{H}^{\mathrm{H}}$. In other words, $\mathrm{ZF}$ aims to minimize the interference. As $\mathrm{ZF}$ requires the matrix inverse, $\left(\mathbf{H H}^{\mathbf{H}}\right)^{-1}$, it is more computationally expensive than the MRC.

\subsubsection{Spectral Efficiency}

Capacity is an upper limit on the data rate which can be archived with arbitrarily low error probability [95, p. 15]. SE is measured in bits/s/Hz and is a lower bound on the capacity that can be achieved by a particular type of processing where any interference is treated as noise [95, p. 14]. In this work, we analyse low-complexity BF techniques, such as MRC in Sec. 3.5.2 and $\mathrm{ZF}$ in Sec. 3.5.2, where SE is a reasonable evaluation metric. SE is defined as $[95$, Eq.1.4, p. 16]

$$
\mathrm{SE}=\log _{2}(1+\mathrm{SINR})
$$

for a fixed channel and a particular type of processing which gives that particular SINR. For fading channels, the mean SE is usually of interest given by $[95$, Eq.1.5, p. 16]

$$
\mathbb{E}[\mathrm{SE}]=\mathbb{E}\left[\log _{2}(1+\mathrm{SINR})\right]
$$




\section{Analysis of Analog MRC for Distributed MU-MIMO Systems}

In this chapter, we investigate the SE performance in distributed systems with an MRC combiner for the uplink and MF for the downlink. The analysis caters for both correlated and line-of-sight (LoS) channels, with the aim of determining and characterising the impact of various antenna layouts. Expected per-user signal power and interference power expressions are derived for both uplink and downlink. Our analysis shows that system performance improves with distributed antenna clusters for digital MRC while analog MRC has a better performance in a centralized system. The impact of the Rice K factor is analysed based on numerical results, and shows that a strong Rice $\mathrm{K}$ factor shrinks the performance gap between analog MRC and digital MRC in a centralized system.

\subsection{Introduction}

MU-MIMO systems have the ability to serve many users over the same timefrequency resource via various beamforming techniques, significantly improving the SE of wireless communication systems. However, high power consumption and space constraints make the implementation of large an- 
tenna arrays difficult. Considering hardware costs, a purely analog architecture at the BS forms an attractive design option. Furthermore, when adding more antennas to a fixed total antenna space, inter-element antenna spacing reduces, and causes spatial correlation between the antenna elements of the array, which degrades system performance, especially for massive MIMO systems. Thus, one design method to mitigate the adverse effects of spatial correlation is to separate the BSs. This is called a collaborative BS [13] design and is also referred to as a distributed system, leading to a different system architecture compared with the centralized or co-located systems. Distributed BSs with large antenna arrays are of great interest as they provide the additional benefit of improved coverage [13] and reduction of spatial correlation relative to large co-located arrays [22]. MRC not only maximizes the received signal power, but also requires no central control $^{1}$ and can be deployed independently at each antenna cluster in a distributed BS layout [22]. Thus, analog MRC is an ideal technique for a large distributed antenna array if the performance is satisfactory.

In recent years, a number of works have analysed the simplest forms of diversity combining techniques, including MRC, equal-gain combining (EGC) and selection combining (SC). Among these, the work in $[96,97]$ shows that EGC is of practical interest as it outperforms SC and has a lower complexity compared to digital MRC. In purely analog MIMO systems, MRC is not feasible. The analog version of MRC is equivalent to EGC as analog processing is not able to change the signal amplitude (see Sec. 3.1). Over the last decade, many works have focused on analog beamforming design and antenna selection methods. Analog beam training approaches have been developed in [15-18]. A novel soft antenna selection approach through RF signal processing has been proposed in [20].

\footnotetext{
${ }^{1}$ MRC only requires the desired user's CSI while ZF needs all users' CSI.
} 
For single-user MIMO, most analytical work concerning analog MRC is conducted from the perspective of modulation, outage probability and bit error probability (BEP) (e.g., [98-104] and references within). The performance of digital MRC in a distributed system is analysed in [22]. For uplink MU-MIMO, a thorough performance analysis of digital MRC over Rayleigh fading without spatial correlation effects is presented in [105]. Approximations of SINR and the ergodic sum SE of a MU-MIMO downlink system using digital MRC with correlation are presented in [106]. Closed-form analyses based on Nakagami-m and gamma fading channels are presented in [107-110]. A closed-from BEP equal gain transmission (EGT) analysis for MUMIMO based on a Nakagami-fading channel is presented in [111] and the energy efficiency of maximum ratio transmission for the downlink is analysed in $[112,113]$. A diversity analysis based on EGT for both single-user MIMO and MU-MIMO was given in [114]. Despite the considerable body of work described above, research work related to analog MRC in Ricean channels is very limited, mainly focusing on system energy efficiency or system sum rate $[115,116]$ in single-user MIMO systems. Thus, we present numerical results adopting a Ricean fading channel to analyse the system performance with respect to different Ricean factors in a MU-MIMO system.

The primary aim of this chapter is to derive SINR results for analog MRC in the uplink and downlink MF in a correlated Rayleigh fading environment for both centralized and distributed MU-MIMO systems. Specifically, a thorough analysis of the expected per-terminal SE based on different BS layouts and the impact of system parameters is of great interest. The contributions of this chapter can be summarized as follows:

- Novel analytical expressions of expected per-user signal power and interference for both uplink MRC and downlink MF are derived. The derivation is robust to changes in system dimension and correlation 
models.

- We analyse the system performance of digital and analog MRC/MF for three BS layouts. We show that while digital MRC benefits from increased BS decentralization, the same does not hold for analog MRC.

- Simulation based investigations of the impact of different Rice K factors on both centralized and distributed systems with analog and digital MRC are presented. We show that the performance of analog MRC approaches that of digital MRC when increasing the $\mathrm{K}$ factor in a centralized system, while the performance gap between the two technique remains in a distributed system.

\subsection{System Model}

We consider a MU-MIMO system with $K$ single antenna users randomly located within a single cell and a total of $N_{\mathrm{t}}$ antennas at the BS divided equally amongst $M$ cooperative antenna groups. Three different BS layouts are adopted for system performance evaluation. Cen (Fig. 4.1) denotes a centralized system where the BS is located at the centre of the cell with all $N_{\mathrm{t}}$ antennas; Dis4 (Fig. 4.2) is a distributed system, where antennas are divided equally amongst four cooperative antenna sites with each site (half way from the centre to the cell-edge and $90^{\circ}$ from each other) equipped with $\frac{N_{t}}{4}$ antennas. Similarly, Dis2 (Fig. 4.3) denotes a system with two sites, each site (half way from the centre to the cell-edge and $180^{\circ}$ from each other) containing $\frac{N_{\mathrm{t}}}{2}$ antennas.

The UL and DL system models are now described. 


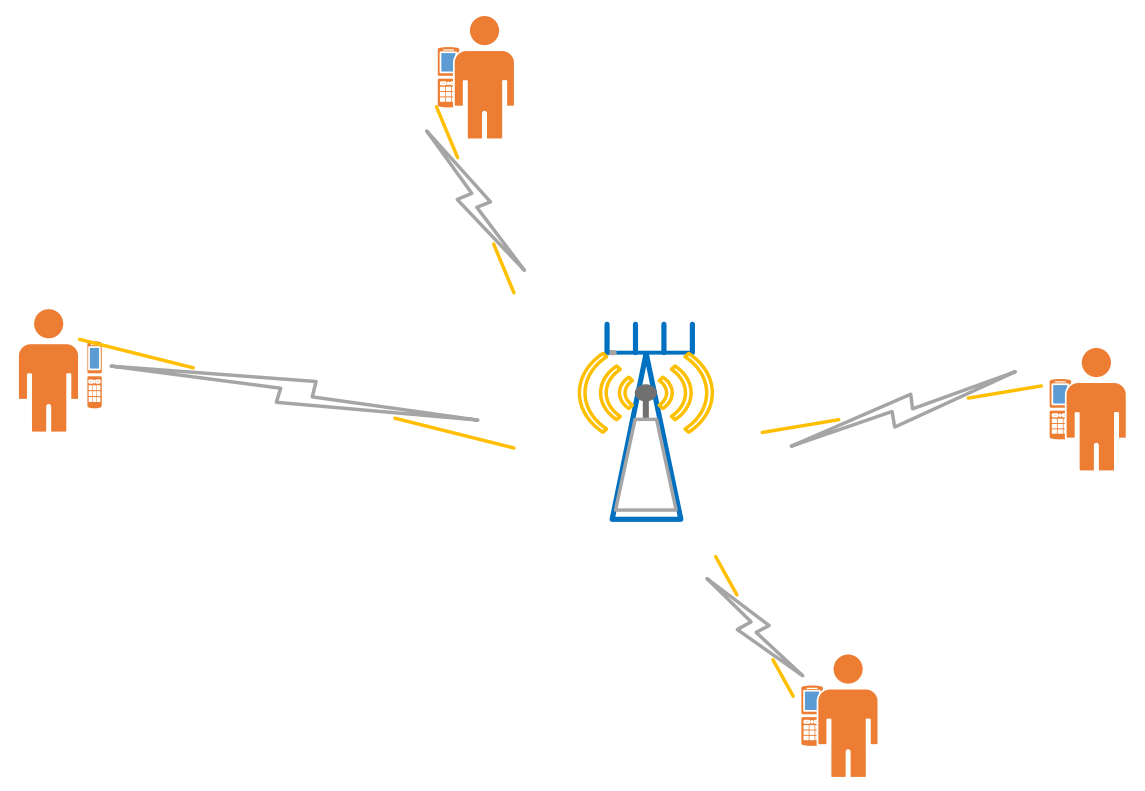

Figure 4.1: Example of a centralized system (Cen) with $N_{t}$ antennas at the central BS and four single antenna users.

\subsubsection{Uplink System Model}

The $N_{\mathrm{t}} \times 1$ channel vector for user $i$ can be written as $\mathbf{h}_{i}=\mathbf{R}_{i}^{\frac{1}{2}} \mathbf{u}_{i}$, where the entries of $\mathbf{u}_{i}$ are independent and identically distributed (i.i.d) Rayleigh fading variables, $\mathbf{u}_{i} \sim \mathcal{C N}(\mathbf{0}, \mathbf{I}) ; \mathbf{R}_{i}$ is the $N_{\mathrm{t}} \times N_{\mathrm{t}}$ spatial correlation matrix for user $i$. Assuming no correlation between antennas belonging to different groups, we write $\mathbf{R}_{i}=\operatorname{diag}\left(\mathbf{R}_{i 1} \mathbf{R}_{i 2} \ldots \mathbf{R}_{i M}\right), \mathbf{R}_{i m}=\bar{\beta}_{i m} \boldsymbol{\Sigma}_{i m}{ }^{2}$, where $i$ indicates the user $i$ and $m$ indicates the base station group $m . \Sigma_{i m}$ contains the correlation coefficients and $\bar{\beta}_{i m}$ models the effect of pathloss and shadowing of the $m^{\text {th }}$ group base

\footnotetext{
${ }^{2}$ The matrix $\mathbf{R}_{i}$ is actually the covariance matrix of the channel, $\mathbf{R}_{i}=\mathbb{E}\left[\mathbf{h}_{i} \mathbf{h}_{i}^{\mathbf{H}}\right]$. Hence, it contains the powers $\left(\bar{\beta}_{i m}\right)$ as well as the correlations $\left(\boldsymbol{\Sigma}_{i m}\right)$. We take out the parameter $\bar{\beta}_{i m}$ so that $\boldsymbol{\Sigma}_{i m}$ is a true correlation matrix with diagonal entries equal to one. This makes analysis easier when using results in [117], which we use extensively. These results are based on unit power Gaussians. Thus, $\boldsymbol{\Sigma}_{i m}$ is more convenient.
} 


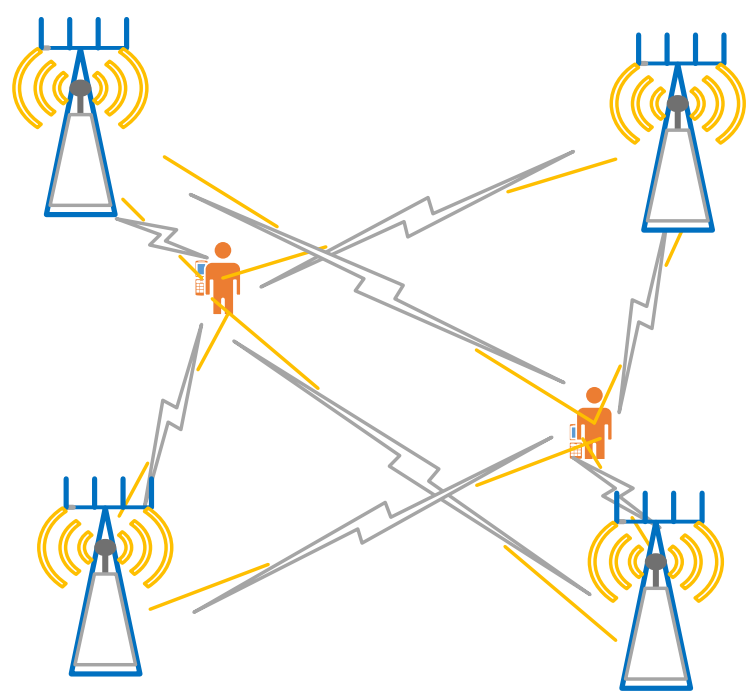

Figure 4.2: Example of a distributed system (Dis4) with four BSs, each BS having $N_{t} / 4$ antennas and two single antenna users.

station for the $i^{\text {th }}$ user. We consider $\bar{\beta}_{i m}=A \zeta_{i m}\left(d_{0} / d_{i m}\right)^{\gamma}$, where $A$ is a unit-less constant indicating the geometric attenuation at the reference distance $d_{0}, d_{i m}$ is the distance between the $i^{\text {th }}$ user and the $m^{\text {th }} \mathrm{BS}$ and $\gamma$ is the pathloss attenuation exponent; $\zeta_{i m}$ is a log-normal random variable, $10 \log _{10} \zeta_{i m} \sim \mathcal{N}\left(0, \sigma_{\text {sh }}^{2}\right)$, to model the effect of shadowing between the the $i^{\text {th }}$ user and the $m^{\text {th }}$ BS. Thus, $\mathbf{H}=\left[\mathbf{h}_{1} \mathbf{h}_{2} \ldots \mathbf{h}_{K}\right]$ denotes the $N_{\mathrm{t}} \times K$ fast-fading channel matrix between $N_{\mathrm{t}}$ antennas at the BS and $K$ end users. Then, under the assumption of perfect channel knowledge at the BS and equal transmit power for each user, for narrow-band transmission, the received signal at the BS can be expressed as

$$
\mathbf{y}=\sqrt{P_{\mathrm{t}}^{\mathrm{ul}}} \mathbf{H} \mathbf{s}+\mathbf{n}_{\mathrm{ul}},
$$




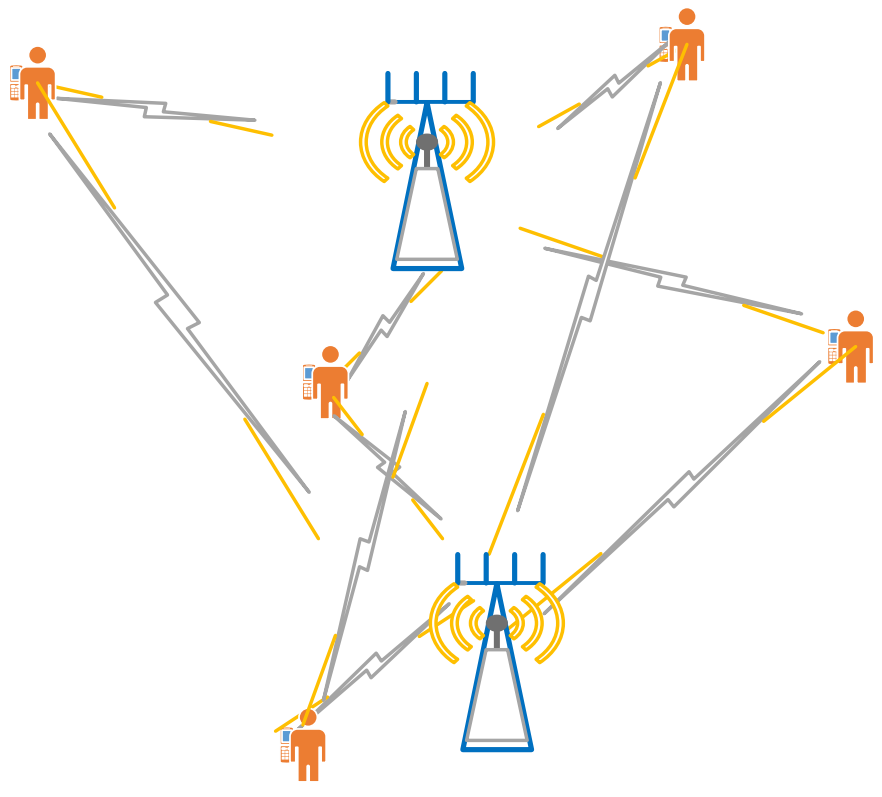

Figure 4.3: Example of a distributed system (Dis2) with two BSs, each BS having $N_{t} / 2$ antennas and four single antenna users.

where $\mathbf{s}$ is the $K \times 1$ data symbol vector from the $K$ end users and $\mathbb{E}\left[\mathbf{s s}^{\mathrm{H}}\right]=\mathbf{I}$; $\mathbf{n}_{\mathrm{ul}}$ models the effect of white Gaussian noise, where the noise variance is assumed to be one; $P_{\mathrm{t}}^{\mathrm{ul}}$ is the uplink transmit power per data stream. After MRC processing, the combined signal for the $i^{\text {th }}$ user at the BS is given by

$$
\mathbf{y}_{i}=\sqrt{P_{\mathrm{t}}^{\mathrm{ul}}} \mathbf{g}_{i}^{\mathrm{H}} \mathbf{h}_{i} s_{i}+\sqrt{P_{\mathrm{t}}^{\mathrm{ul}}} \sum_{\substack{l=1 \\ l \neq i}}^{K} \mathbf{g}_{i}^{\mathrm{H}} \mathbf{h}_{l} s_{l}+\mathbf{g}_{i}^{\mathrm{H}} \mathbf{n}_{\mathrm{ul}},
$$

where $\mathbf{g}_{i}=\mathbf{h}_{i}$ for digital MRC and $\mathbf{g}_{i}=\hat{\mathbf{h}}_{i}, \hat{\mathbf{h}}_{i}=\exp \left(j \angle \mathbf{h}_{i}\right)$ for analog MRC, where $\angle \mathbf{h}_{i}$ represents the vector of angles of each element of $\mathbf{h}_{i}$. This results 
in the SINR for user $i$ given by

$$
\operatorname{SINR}_{i}=\frac{P_{\mathrm{t}}^{\mathrm{ul}}\left|\mathbf{g}_{i}^{\mathrm{H}} \mathbf{h}_{i}\right|^{2}}{P_{\mathrm{t}}^{\mathrm{ul}}\left\{\sum_{\substack{l=1 \\ l \neq i}}^{K}\left|\mathbf{g}_{i}^{\mathrm{H}} \mathbf{h}_{l}\right|^{2}\right\}+\mathbf{g}_{i}^{\mathrm{H}} \mathbf{g}_{i}}
$$

\subsubsection{Downlink System Model}

The $K \times N_{\mathrm{t}}$ fast-fading channel matrix for the downlink can be written as $\mathbf{H}=\left[\mathbf{h}_{1}^{\mathrm{T}} \mathbf{h}_{2}^{\mathrm{T}} \ldots \mathbf{h}_{K}^{\mathrm{T}}\right]^{\mathrm{T}}$, where the $1 \times N_{\mathrm{t}}$ channel vector for the $i^{\text {th }}$ user is $\mathbf{h}_{i}=\mathbf{u}_{i} \mathbf{R}_{i}^{\frac{1}{2}}$, and $\mathbf{u}_{i}$ and $\mathbf{R}_{i}$ are defined in Section 4.2.1 with the exception that $\mathbf{u}_{i}$ is now $1 \times M$. The received signal at the $i^{\text {th }}$ user can be expressed as

$$
\mathbf{y}_{i}=\sqrt{P_{\mathrm{t}}^{\mathrm{dl}}} \mathbf{h}_{i} \mathbf{g}_{i}^{\mathrm{H}} s_{i}+\sqrt{P_{\mathrm{t}}^{\mathrm{d}}} \sum_{\substack{l=1 \\ l \neq i}}^{K} \mathbf{h}_{i} \mathbf{g}_{l}^{\mathrm{H}} s_{l}+n_{\mathrm{dl}}
$$

where $P_{\mathrm{t}}^{\mathrm{dl}}$ is the downlink transmit power per data stream, $n_{\mathrm{dl}}$ models the effect of white Gaussian noise with unit variance, and $\mathbf{g}_{i}=\frac{\mathbf{h}_{i}}{\left\|\mathbf{h}_{i}\right\|}$ for digital MRC and $\mathbf{g}_{i}=\frac{\hat{\mathbf{h}}_{i}}{\sqrt{N_{\mathrm{t}}}}$ for analog MRC. Thus, the corresponding SINR for user $i$ is given by

$$
\operatorname{SINR}_{i}=\frac{P_{\mathrm{t}}^{\mathrm{dl}}\left|\mathbf{h}_{i} \mathbf{g}_{i}^{\mathrm{H}}\right|^{2}}{\left.P_{\mathrm{t}}^{\mathrm{dl}}\left\{\sum_{\substack{l=1 \\ l \neq i}}^{K}\left|\mathbf{h}_{i} \mathbf{g}_{l}^{\mathrm{H}}\right|^{2}\right]\right\}+1}
$$

\subsection{Approximations of Achievable Uplink and Down- link Spectral Efficiency}

In this section, we derive expressions for the expected per-user signal power and interference power for both uplink and downlink analog MRC systems. This is used to enable analysis of uplink and downlink achievable rates. For 
example, the achievable uplink rate is given by

$$
\left[\mathrm{R}_{\mathrm{ul}}\right] \approx \log _{2}\left\{1+\frac{\frac{P_{\mathrm{t}}^{\mathrm{ul}}}{N_{\mathrm{t}}}\left[\left|\mathbf{w}_{i}^{\mathrm{H}} \mathbf{h}_{i}\right|^{2}\right]}{\frac{P_{\mathrm{t}}^{\mathrm{ul}}}{N_{\mathrm{t}}}\left[\sum_{\substack{l=1 \\ l \neq i}}^{K}\left|\mathbf{w}_{i}^{\mathrm{H}} \mathbf{h}_{l}\right|^{2}\right]+1}\right\}
$$

To enable the analysis, we apply a commonly used approximation as follows: if $X=\sum X_{i}$ and $Y=\sum Y_{i}$ are both sums of non-negative random variables, then $\mathbb{E}\left[\log _{2}\left(1+\frac{X}{Y}\right)\right] \approx \log _{2}\left(1+\frac{\mathbb{E}[X]}{\mathbb{E}[Y]}\right)$ [105]. Independence between $X$ and $Y$ is not required and the result becomes more accurate when the number of the summation terms in $X$ and $Y$ is large [105]. This behaviour is due to the law of large numbers [105] where both the numerator and denominator approach their mean values (after normalising) and their variances become small. For the uplink system, substituting the analog MRC precoder into (4.3) gives the approximation of per-user spectral efficiency as

$$
\mathbb{E}\left[\mathbf{R}_{\mathrm{ul}}\right] \approx \log _{2}\left\{1+\frac{\frac{P_{\mathrm{t}}^{\mathrm{ul}}}{N_{\mathrm{t}}} \mathbb{E}\left[\left|\hat{\mathbf{h}}_{i}^{\mathrm{H}} \mathbf{h}_{i}\right|^{2}\right]}{\frac{P_{\mathrm{t}}^{\mathrm{ul}}}{N_{\mathrm{t}}} \mathbb{E}\left[\sum_{\substack{l=1 \\ l \neq i}}^{K}\left|\hat{\mathbf{h}}_{i}^{\mathrm{H}} \mathbf{h}_{l}\right|^{2}\right]+1}\right\}
$$

whereas for the downlink, we have

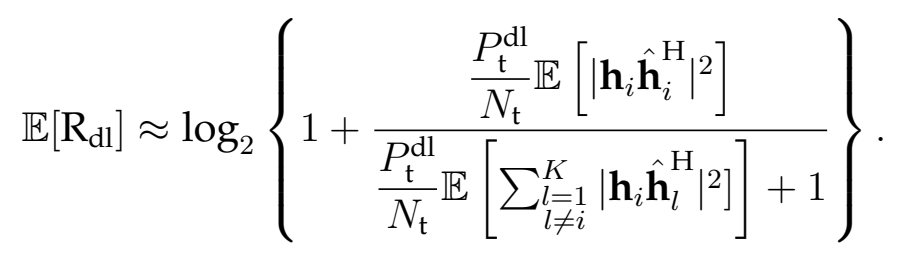

The achievable rates in (4.7) and (4.8) give the UL and DL SE values which are particularly useful as they link the achievable rates to particular types of 
processing. While capacity is the ultimate limit on error-free rate it assumes the ability to employ processing of any complexity. In contrast, the SE gives a rate which is achievable for practical processing schemes such as $\mathrm{MF}, \mathrm{ZF}$, MMSE, etc. While simulations can always be used to simulate the SEs, we prefer to seek analytical approximations as these may able to provide further insights. For example, we use the analytical results in Sec. 4.3.2 to explain why equal correlation is the worst case. Also, in other chapters, many insights are derived from analytical results, such as Sec. 5.3, Sec. 6.3.2 and Theorem 1 in Chapter 7. We now derive the expectation of the signal and interference terms in Sec. 4.3.1 and Sec. 4.3.2, respectively.

\subsubsection{Expected Signal Power}

We derive the expected signal power for analog MRC on the uplink in detail, while for the downlink we only give the final result as a similar analysis applies. The expected signal power for the uplink can be derived as follows,

$$
\begin{aligned}
& \mathbb{E}\left[\left|\hat{\mathbf{h}}_{i}^{\mathrm{H}} \mathbf{h}_{i}\right|^{2}\right]=\mathbb{E}\left[\left|\sum_{j=1}^{N} \hat{h}_{i j}^{*} h_{i j}\right|^{2}\right]=\mathbb{E}\left[\sum_{j=1}^{N}\left|h_{i j}\right|^{2}\right]+\mathbb{E}\left[\sum_{\substack{j=1 \\
j \neq k}}^{N} \sum_{\substack{k=1 \\
j}}^{N} h_{i j}|| h_{i k} \mid\right],
\end{aligned}
$$

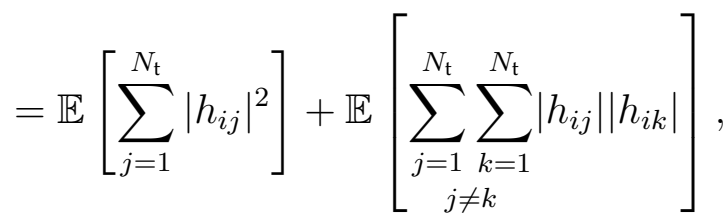

$$
\begin{aligned}
& =\sum_{j=1}^{N_{\mathrm{t}}} \beta_{i j}+\sum_{\substack{j=1 \\
j \neq k}}^{N_{\mathrm{t}}} \sum_{\substack{k=1 \\
N_{\mathrm{t}}}} \sqrt{\beta_{i j} \beta_{i k}} \mathbb{E}\left[\left|v_{i j}\right|\left|v_{i k}\right|\right],
\end{aligned}
$$


where $\beta_{i j}$ is the link gain from the $i^{\text {th }}$ user to the $j^{\text {th }}$ antenna and $h_{i j}=\sqrt{\beta_{i j}} v_{i j}$. In (6.7), $v_{i j}$ and $v_{i k}$ are two correlated complex Gaussian variables, and can be rewritten as $v_{i j}=r_{1} \exp \left(j \theta_{1}\right)$ and $v_{i k}=r_{2} \exp \left(j \theta_{2}\right)$. Thus $\left|v_{i j}\right|=r_{1}$ and $\left|v_{i k}\right|=r_{2}$. From [117, p. 97], we know that,

$$
\mathbb{E}\left[r_{1} r_{2}\right]=\frac{\pi}{4 \sqrt{\left(S_{11} S_{22}\right)}}\left(1-\lambda_{12}^{2}\right)_{2} F_{1}\left(\frac{3}{2}, \frac{3}{2}, 1 ; \lambda_{12}^{2}\right),
$$

where ${ }_{2} F_{1}$ is the Gaussian (or ordinary) hypergeometric function; $S_{11}$ and $S_{22}$ are entries of the inverse covariance matrix, $\mathbf{S}$, of $v_{i j}$ and $v_{i k}$, where

$$
\mathbf{S}=\left[\begin{array}{ll}
S_{11} & S_{12} \\
S_{21} & S_{22}
\end{array}\right]=\left[\begin{array}{cc}
\frac{1}{1-|\rho|^{2}} & \frac{-\rho}{1-|\rho|^{2}} \\
\frac{-\rho^{*}}{1-|\rho|^{2}} & \frac{1}{1-|\rho|^{2}}
\end{array}\right]
$$

Also, $\lambda_{12}^{2}=\frac{\left|S_{12}\right|^{2}}{S_{11} S_{22}}=|\rho|^{2}$ and $\rho$ is the correlation between $v_{i j}$ and $v_{i k}$. Applying this result gives

$$
\mathbb{E}\left[\left|v_{i j}\right|\left|v_{i k}\right|\right]=\frac{\pi}{4}\left(1-\left|\rho_{i j k}\right|^{2}\right)_{2}^{2} F_{1}\left(\frac{3}{2}, \frac{3}{2}, 1 ;\left|\rho_{i j k}\right|^{2}\right),
$$

where $\rho_{i j k}$ is the correlation obtained from the $j k^{\text {th }}$ entry of $\mathbf{R}_{i}$. When $j$ and $k$ correspond to different BS groups, there is no correlation, $\rho_{i j k}=0$, and

$$
\mathbb{E}\left[\left|v_{i j}\right|\left|v_{i k}\right|\right]=\mathbb{E}\left[\left|v_{i j}\right|\right]^{2}=\Gamma\left(\frac{3}{2}\right)^{2}
$$

Hence, the final result is, 


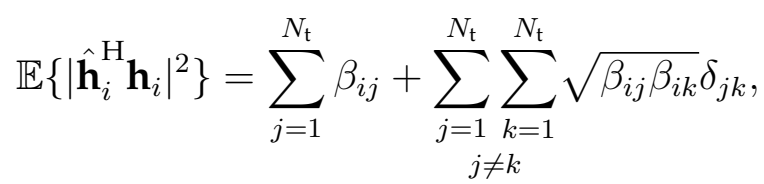

where

$$
\delta_{j k}= \begin{cases}\Gamma\left(\frac{3}{2}\right)^{2}, & j, k \notin B_{k} \\ \frac{\pi}{4}\left(1-\left|\rho_{i j k}\right|^{2}\right)^{2}{ }_{2} F_{1}\left(\frac{3}{2}, \frac{3}{2}, 1 ;\left|\rho_{i j k}\right|^{2}\right), & j, k \in B_{k}\end{cases}
$$

and $B_{k}$ is the set of antennas at the same site as antenna $k$. The expected signal power can be derived similarly for the downlink, resulting in

$$
\mathbb{E}\left\{\left|\mathbf{h}_{i} \hat{\mathbf{h}}_{i}^{\mathrm{H}}\right|^{2}\right\}=\sum_{j=1}^{N_{\mathrm{t}}} \beta_{i j}+\sum_{\substack{j=1 \\ j \neq k}}^{N_{\mathrm{t}}} \sum_{\substack{k=1 \\ N_{\mathrm{t}}}}^{\sqrt{\beta_{i j} \beta_{i k}}} \delta_{j k}
$$

where

$$
\delta_{j k}= \begin{cases}\Gamma\left(\frac{3}{2}\right)^{2}, & j, k \notin B_{k} \\ \frac{\pi}{4}\left(1-\left|\rho_{i j k}\right|^{2}\right)^{2}{ }_{2} F_{1}\left(\frac{3}{2}, \frac{3}{2}, 1 ;\left|\rho_{i j k}\right|^{2}\right), & j, k \in B_{k}\end{cases}
$$

\subsubsection{Expected Interference Power}

The interference power for the uplink from the $l^{\text {th }}$ user to the $i^{\text {th }}$ user can be derived as follows, 


$$
\begin{aligned}
\mathbb{E}\left[\left|\hat{\mathbf{h}}_{i}^{\mathrm{H}} \mathbf{h}_{l}\right|^{2}\right] & =\mathbb{E}\left\{\left|\left(\hat{h}_{i 1}^{*} \hat{h}_{i 2}^{*} \ldots \hat{h}_{i N}^{*}\right)\left(h_{l 1} h_{l 2} \ldots h_{l N}\right)^{T}\right|^{2}\right\} \\
& =\mathbb{E}\left\{\hat{\mathbf{h}}_{i}^{\mathrm{H}} \mathbf{h}_{l} \mathbf{h}_{l}^{\mathrm{H}} \hat{\mathbf{h}}_{i}\right\} \\
& =\mathbb{E}\left[\hat{\mathbf{h}}_{i}^{\mathrm{H}} \mathbb{E}\left[\mathbf{R}_{l}^{\frac{1}{2}} \mathbf{u}_{l} \mathbf{u}_{l}^{\mathrm{H}} \mathbf{R}_{l}^{\frac{1}{2}}\right] \hat{\mathbf{h}}_{i}\right] \\
& =\mathbb{E}\left[\hat{\mathbf{h}}_{i}^{\mathrm{H}} \mathbf{R}_{l} \hat{\mathbf{h}}_{i}\right] \\
& =\mathbb{E}\left\{\left(\frac{h_{i 1}^{*}}{\left|h_{i 1}\right|} \frac{h_{i 2}^{*}}{\left|h_{i 2}\right|} \ldots \frac{h_{i N}^{*}}{\left|h_{i N}\right|}\right) \mathbf{R}_{l}\left(\frac{h_{i 1}}{\left|h_{i 1}\right|} \frac{h_{i 2}}{\left|h_{i 2}\right|} \ldots \frac{h_{i N}}{\left|h_{i N}\right|}\right)^{T}\right\} \\
& =\mathbb{E}\left[\sum_{j=1}^{N_{\mathrm{t}}} \sum_{k=1}^{N_{\mathrm{t}}} \frac{h_{i j}^{*}}{\left|h_{i j}\right|}\left(\mathbf{R}_{l}\right)_{k j}\left(\frac{h_{i k}}{\left|h_{i k}\right|}\right)\right] \\
& =\sum_{j=1}^{N_{\mathrm{t}}} \sum_{k=1}^{N_{\mathrm{t}}}\left(\mathbf{R}_{l}\right)_{k j} \mathbb{E}\left[\frac{v_{i j}^{*}}{\left|v_{i j}\right|} \frac{v_{i k}}{\left|v_{i k}\right|}\right] .
\end{aligned}
$$

Reusing the notation, $v_{i j}=r_{1} \exp \left(j \theta_{1}\right)$ and $v_{i k}=r_{2} \exp \left(j \theta_{2}\right)$, we have $\mathbb{E}\left[\frac{v_{i j}^{*}}{\left|v_{i j}\right|} \mid \frac{v_{i k}}{\left|v_{i k}\right|}\right]=$ $\mathbb{E}\left[e^{j\left(\theta_{1}-\theta_{2}\right)}\right]$. From [117, p. 100],

$$
\mathbb{E}\left\{e^{j\left(\theta_{1}-\theta_{2}\right)}\right\}=\frac{\pi}{4} \rho\left(1-\rho^{2}\right)_{2} F_{1}\left(\frac{3}{2}, \frac{3}{2}, 2 ;|\rho|^{2}\right)
$$

Thus,

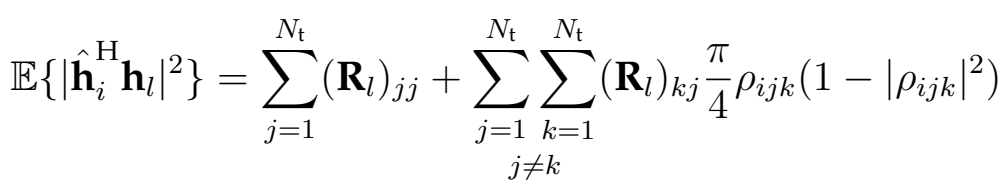

$$
\begin{aligned}
& \times{ }_{2} F_{1}\left(\frac{3}{2}, \frac{3}{2}, 2 ;\left|\rho_{i j k}\right|^{2}\right),
\end{aligned}
$$

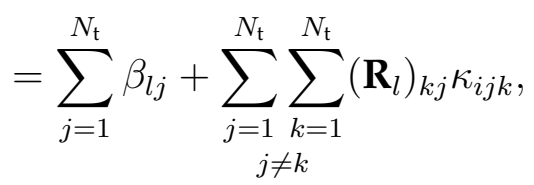


where

$$
\kappa_{i j k}= \begin{cases}0, & j, k \notin B_{k} \\ \frac{\pi}{4} \rho_{i j k}\left(1-\left|\rho_{i j k}\right|^{2}\right)_{2} F_{1}\left(\frac{3}{2}, \frac{3}{2}, 2 ;\left|\rho_{i j k}\right|^{2}\right), & j, k \in B_{k} .\end{cases}
$$

Similarly, the downlink expected interference power is,

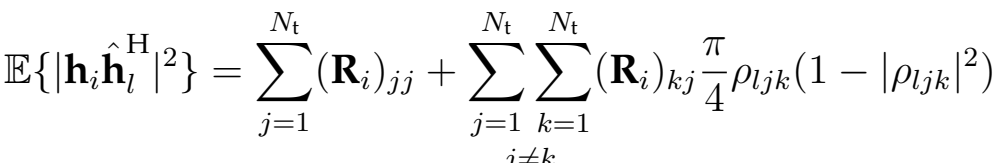

$$
\begin{aligned}
& \times_{2} F_{1}\left(\frac{3}{2}, \frac{3}{2}, 2 ;\left|\rho_{l j k}\right|^{2}\right) \text {, }
\end{aligned}
$$

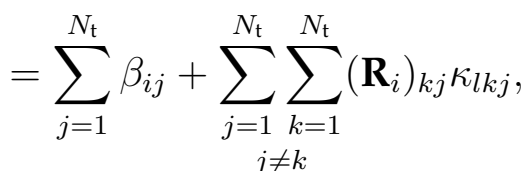

where

$$
\kappa_{l k j}= \begin{cases}0, & j, k \notin B_{k}, \\ \frac{\pi}{4} \rho_{l j k}\left(1-\left|\rho_{l j k}\right|^{2}\right)_{2} F_{1}\left(\frac{3}{2}, \frac{3}{2}, 2 ;\left|\rho_{l j k}\right|^{2}\right), & j, k \in B_{k} .\end{cases}
$$

Thus, substituting (4.14), (4.15), (4.20) and (4.21) into (4.3), we obtain the per-user spectral efficiency for the uplink. Similarly substituting (4.14), (4.15), (4.22) and (4.23) into (4.5), we obtain the per-user spectral efficiency for the downlink. Not only do (4.20) and (4.22) provide the value of uplink and downlink interference, they also provide an important insight into MU-MIMO with analog processing. When the users all have the same correlation matrix, then $\left(\mathbf{R}_{l}\right)_{k j} \times \rho_{i j k}=\left|\rho_{i j k}\right|^{2}>0$ and $\left(\mathbf{R}_{i}\right)_{k j} \times \rho_{l j k}=\left|\rho_{l j k}\right|^{2}>0$, which maximizes the interference in (4.20) and (4.22) as all summation terms are posi- 
tive. Hence, equal correlation matrices is the worst case, as shown in [106] for digital MRC. This is the first demonstration of this property for analog processing.

\subsection{Numerical Results}

In this section, numerical results with the following parameters are presented. Four users each with a single antenna are uniformly located in a circular cell with the radius of 100 meters, as depicted in Fig. 2.1 and Fig. 2.2. The unitless geometric attenuation is $A=30 \mathrm{~dB}$ and the reference distance is $d_{0}=1$ meter. The total number of antennas $N_{\mathrm{t}}=32$; the pathloss attenuation $\gamma=3.5$ and the standard deviation of shadowing $\zeta=6 \mathrm{~dB}$. The transmit power $P_{t}$ is chosen to guarantee that $95 \%$ of the time the SNR, defined by the ratio of the received power to the noise power in a single-user single-antenna system, exceeds $0 \mathrm{~dB}$. As our approximations of SINR are accurate for arbitrary correlation models, two popular correlation models are considered. For the exponential correlation model in [118], the correlation matrices required for user $i$ are defined by $\left(\Sigma_{i m}\right)_{r s}=\left[\rho \exp \left(j \phi_{i m}\right)\right]^{|r-s|}$. Here, $\rho$ is the common magnitude of the correlation between adjacent antennas and $\phi_{i m} \sim U[0,2 \pi]$ is a user specific phase at each antenna group. We also consider the one-ring correlation model [57], with an angle spread of $30^{\circ}$ and a different central azimuth angle with a uniform distribution within $[0,2 \pi]$ for each user at each antenna group. We refer to this model as OR.uni. As LoS will play an important role in upcoming systems, we are also interested in the impact of Rice $K$ factors on the BS layouts. The model for the uplink Ricean channel for the $i^{\text {th }}$ user can be written as

$$
\mathbf{h}_{i}=\sqrt{\frac{K_{i}}{K_{i}+1}} \overline{\mathbf{u}}_{i}+\sqrt{\frac{1}{K_{i}+1}} \mathbf{R}_{i}^{\frac{1}{2}} \mathbf{u}_{i}
$$


where $K_{i}$ is the Rice factor, $\overline{\mathbf{u}}_{i}$ is the specular (LoS) component and $\mathbf{u}_{i}\left(N_{\mathrm{t}} \times 1\right)$ is the diffuse (scattered) component. $\overline{\mathbf{u}}_{i}\left(N_{\mathrm{t}} \times 1\right)$ is governed by the transmit and receive array response vectors [42]. As we only consider a uniform linear array (ULA) with a single antenna at the receiver, the uplink LoS vector can be written as, $\overline{\mathbf{u}}_{i}=\mathbf{u}_{\mathrm{tx}}\left(\theta_{i}^{\mathrm{tx}}\right), \mathbf{u}_{\mathrm{tx}}\left(\theta_{i}^{\mathrm{tx}}\right)=\left[1, e^{j 2 \pi d \cos \left(\theta_{i}\right)}, e^{j 2 \pi d 2 \cos \left(\theta_{i}\right)}, \ldots, e^{j 2 \pi d\left(N_{\mathrm{t}}-1\right) \cos \left(\theta_{i}\right)}\right]^{\mathrm{T}}$, where $d$ is the normalized antenna spacing, assumed to be half the carrier wavelength. Finally, $\theta_{i}$ is the angle of arrival, $\theta_{i} \sim U[0,2 \pi]$.

Fig. 4.4 illustrates the CDF of the uplink expected per-user spectral efficiency based on the Dis4 system architecture with three different correlation models. The expectation is computed over the fast-fading and the CDF depicts the impact of the variations in large-scale fading link gains. Exp.0.95fixed denotes the Clarke correlation model [55] with a fixed correlation coefficient $\rho=0.95$ and no random phase; OR.uni denotes the one-ring correlation model; Exp0.7random denotes the exponential correlation with uniformly distributed phase on $[0,2 \pi]$ and $\rho=0.7$. In this figure we compare the CDF of the derived analog per-user spectral efficiency approximation with its simulated counterparts. As we can see, the derived approximations are tight for all three correlation models considered.

In Fig. 4.5 we present the performance comparison of analog and digital MRC for three system layouts: Cen, Dis2, Dis4. Using the same propagation parameters as in Fig. 4.4 and the OR.uni correlation model, the CDFs of peruser spectral efficiency are shown. Generally, digital MRC outperforms ana$\log$ MRC in all three systems. This is mainly due to the fact that analog MRC is incapable of changing the amplitude of the incoming signals. For digital $\mathrm{MRC}$, the system benefits from greater antenna distribution. However, the trend is opposite for analog MRC, where Dis2 and Cen have almost the same performance and both outperform Dis4. Digital MRC benefits from a more distributed system where there is a higher probability of a strong link gain to 


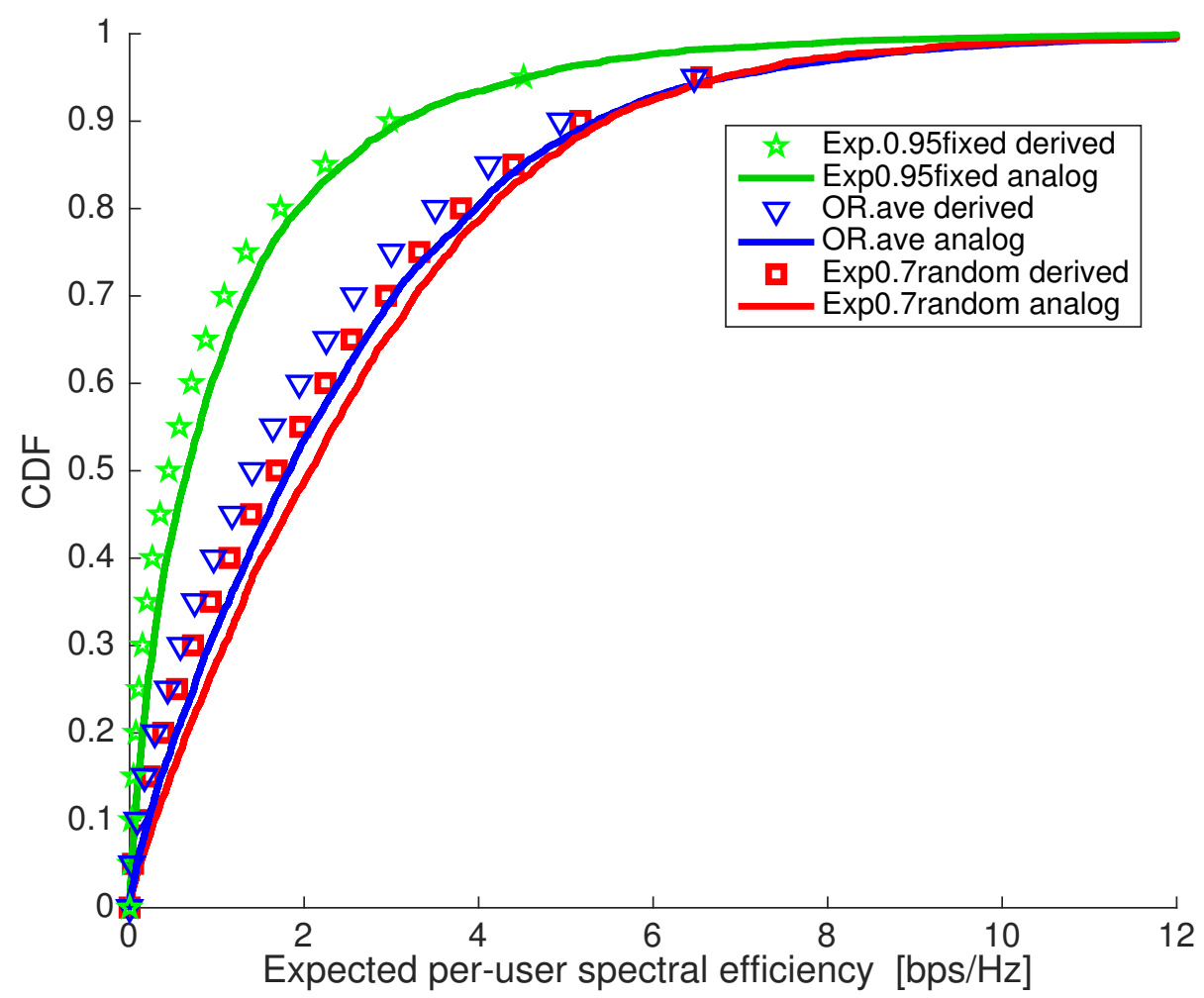

Figure 4.4: Expected per-user spectral efficiency CDFs of analog MRC for an uplink Dis4 system with three correlation models.

one of the antenna clusters. Analog MRC, however, is not able to benefit the users by optimising the receive SNR over different link gains. This is because analog MRC is equivalent to equal gain combining. Thus the centralized system results in the strongest signal power compared with distributed systems when adopting analog MRC processing.

We show numerical results for the Ricean channels for the Cen and Dis4 systems in Fig. 4.6 and Fig. 4.7, respectively. The trends in Fig. 4.6 and Fig. 4.7 illustrate that increasing the Rice K factors can actually increase per-user spectral efficiency in both Cen and Dis4 systems as LoS reduces channel fading fluctuations and also the MRC inter-user-interference as shown in [105]. The gap between analog and digital MRC diminishes to zero in a Cen system when 


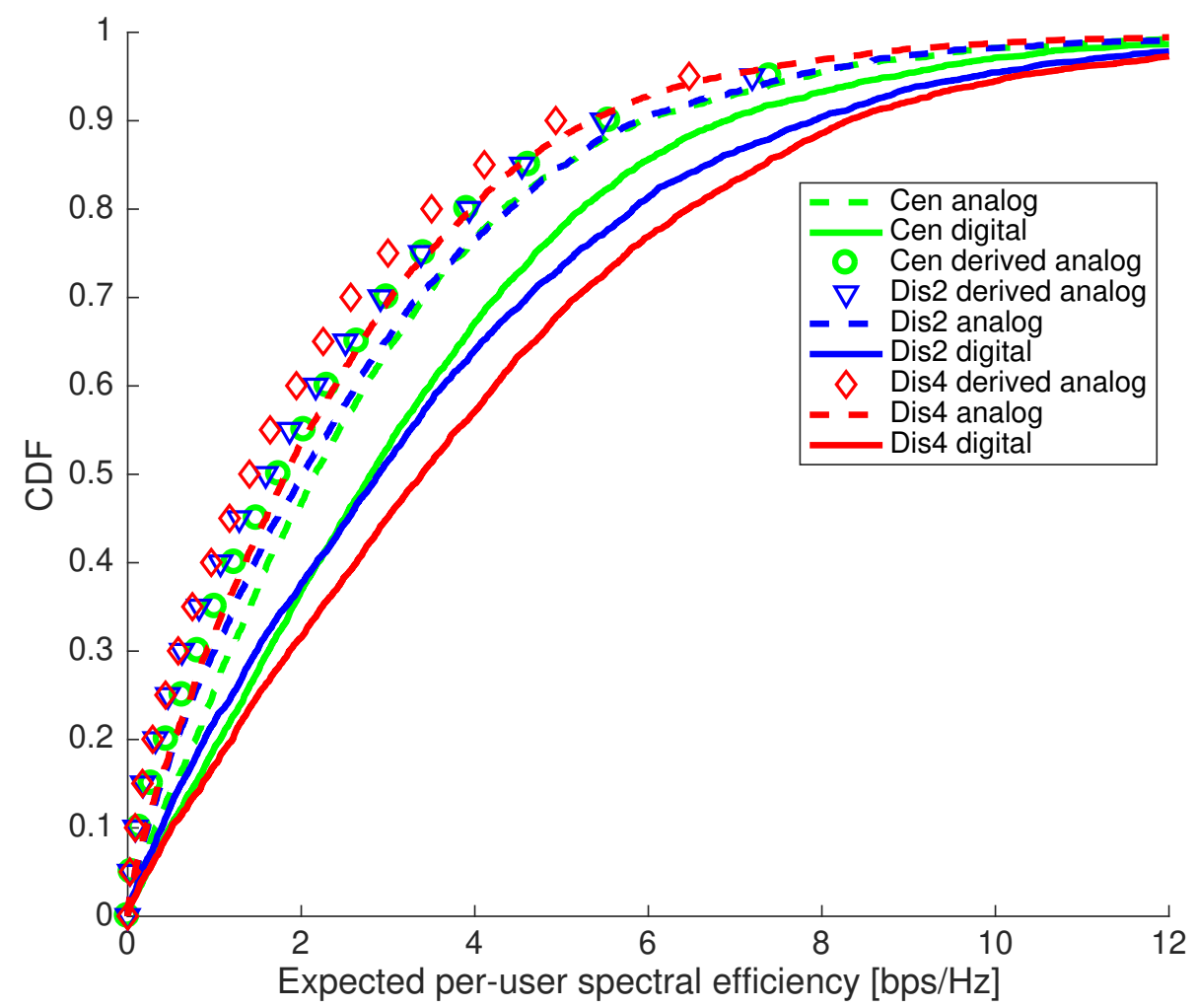

Figure 4.5: Performance comparison of expected per-user spectral efficiency CDFs between analog and digital MRC for uplink.

the Rice $\mathrm{K}$ is greater than $5 \mathrm{~dB}$, where the strong LoS results in low diversity of signals for which analog and digital MRC have almost the same performance. This is not the case for the Dis4 system due to the link gain diversity from which digital MRC benefits.

Finally, in Fig. 4.8 we consider the downlink system. The figure not only shows the accuracy of the approximations, but also that digital MRC in Dis4 significantly outperforms the Cen system. This is mainly due to the high diversity of link gains in a distributed system as the per-user spectral efficiency is affected by the desired user's link gains at different sites. Examining the derivations of (4.14) and (4.22), both the numerator and denominator of the derived SINR for the downlink are affected by the same desired user's link 


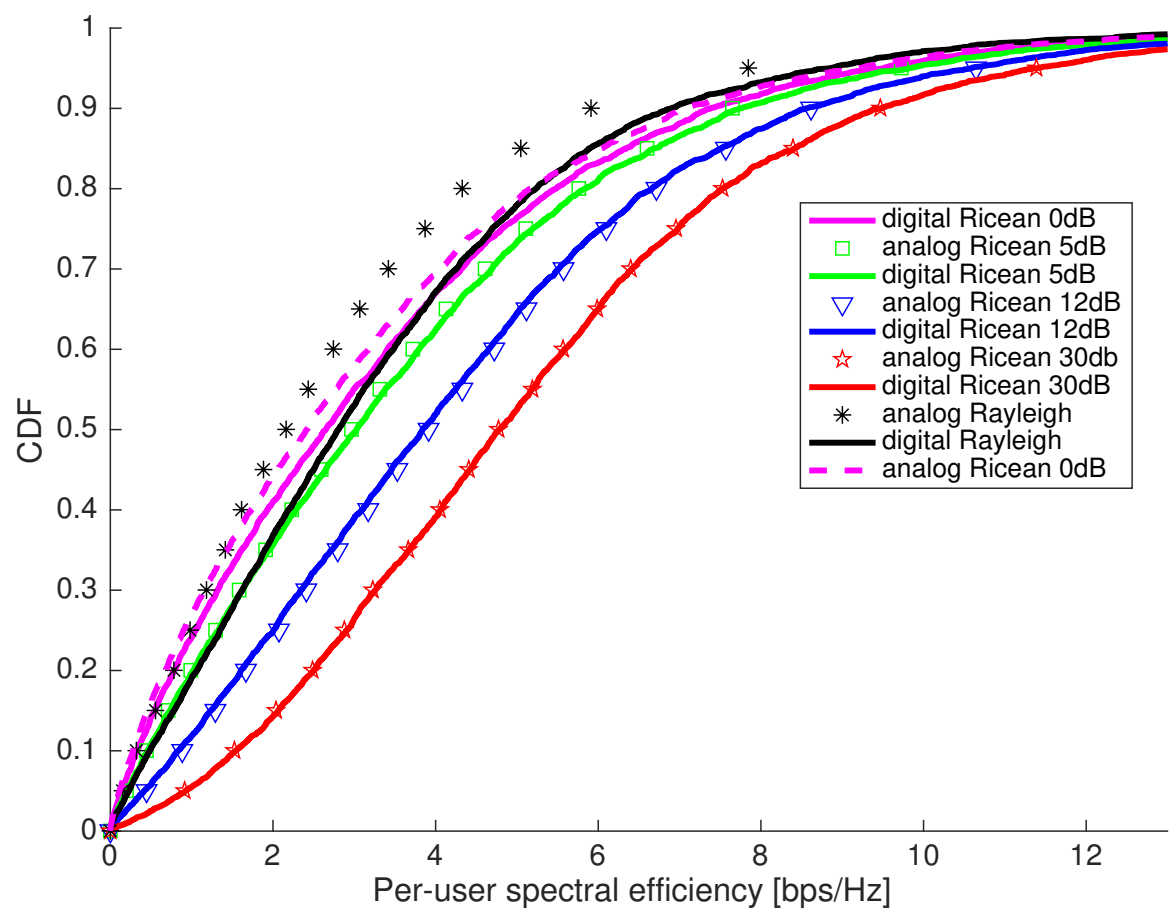

Figure 4.6: Expected per-user spectral efficiency CDFs of analog and digital MRC for an uplink Cen system.

gain, $\bar{\beta}_{i m}$, which is the same for both the numerator and denominator in the centralized system. Dividing by $\bar{\beta}_{i m}$ for both the numerator and denominator in (4.5), we see that variations in the CDF of the expected per-user SINR are limited, reflected in Fig. 4.8 by the steep curves of the Cen system. On the other hand, in the distributed system $\bar{\beta}_{i m}$ takes on different values for the numerator and denominator of the SINR, leading to more diversity in the Dis4 system for digital MRC. For analog processing, the CDFs for the centralized and distributed cases have a cross-over, with distributed being better at low SINR and centralized being better at high SINR. At the low end this is due to the advantage of distributed processing in having power diversity at the different locations which helps to limit the cases where the signal strength is low at every location. At the high end, when the centralized location has very good signal strength, centralized processing has a greater SINR as the dis- 


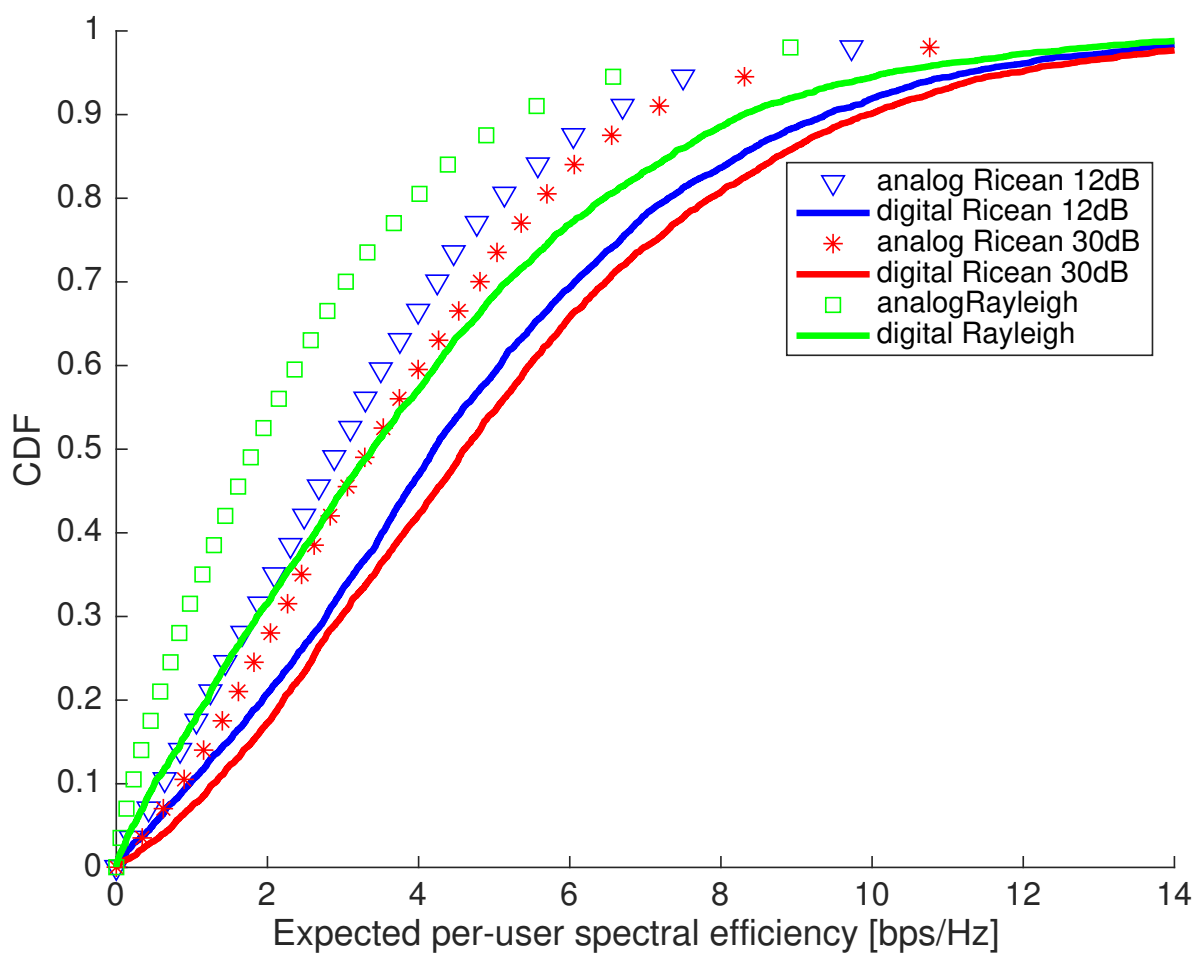

Figure 4.7: Expected per-user spectral efficiency CDFs of analog and digital MRC for an uplink Dis4 system.

tributed processing will average over the other locations which are likely to have lower strength. Note that this is true for analog processing as it cannot make use of the variations in signal strength. Another cross-over in Fig. 4.8 is between distributed analog and centralized digital processing. Usually, digital processing is better, but at the low end we have the same property discussed above. The distributed analog system can outperform centralized digital processing due to the protection of multiple locations which avoids the occasional very low signal strength which can occur in the centralized case. 


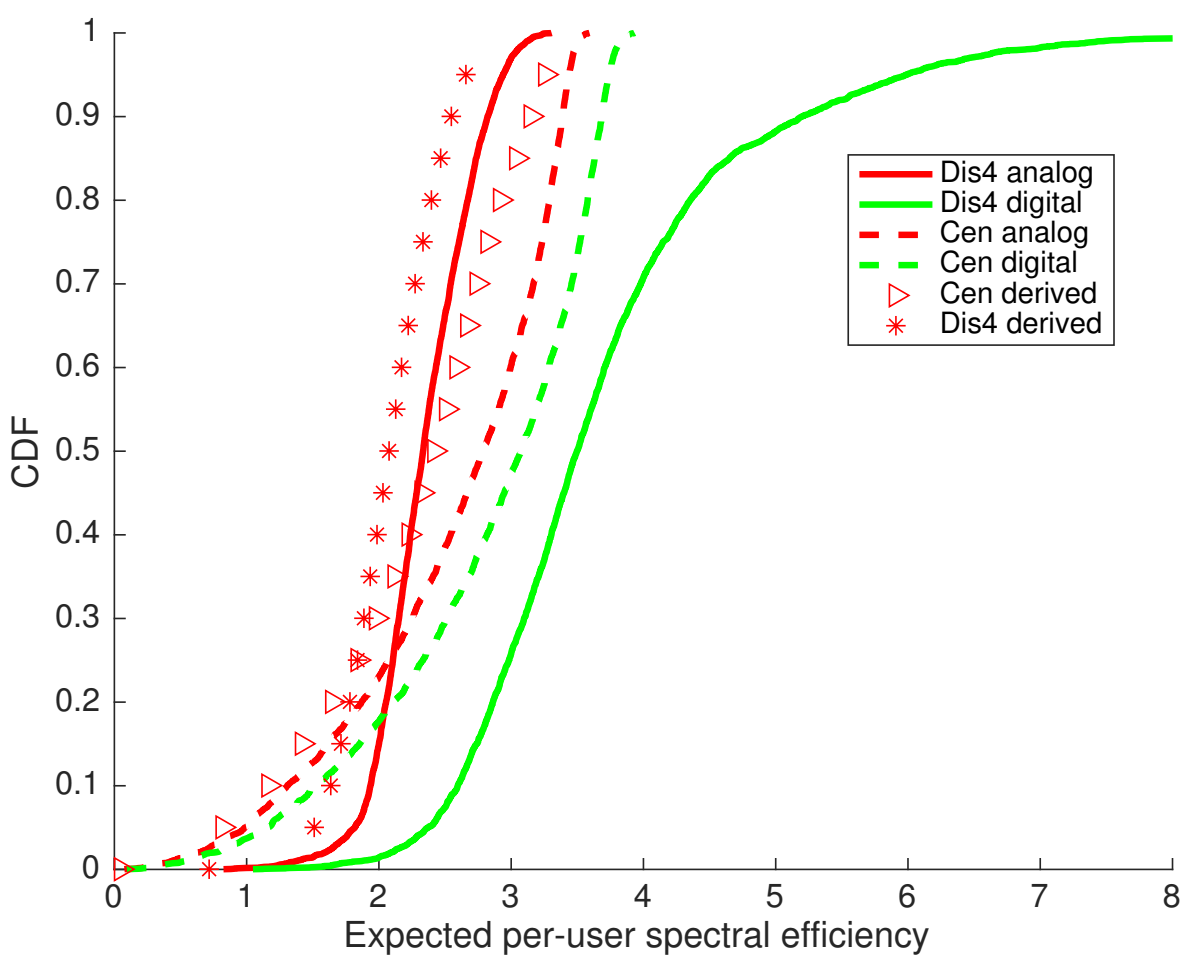

Figure 4.8: Expected per-user spectral efficiency CDFs of analog and digital MRC for downlink Dis4 and Cen systems. Derived results are for the analog case.

\subsection{Conclusion}

We have derived approximations to per-user SINR for analog MRC/MF for uplink/downlink systems, leading to an insightful comparison of analog and digital processing for three different BS layouts. While digital MRC benefits from increased BS decentralization, the same does not hold for analog MRC. We present an analysis of the impact of different Rice $\mathrm{K}$ factors on both centralized and distributed systems with analog and digital MRC technique. When increasing the $\mathrm{K}$ factor in a centralized system, the performance of ana$\log$ MRC approaches that of digital MRC, while the performance gap between the two technique remains in a distributed system. Analog MRC is a compro- 
mise technique for massive MIMO when digital MRC is either expensive or complicated for system design. 


\section{Analysis of Analog and Digital MRC in Massive MU-MIMO Systems over Correlated Channels}

While digital multi-user MRC (MU-MRC) is well understood, relatively few analytical results exist for analog MU-MRC. For example, it was recently shown that MU system performance is highly dependent on the correlation model used [119], but the scope of this work is limited to digital processing (including MRC). Thus, in this chapter, we look into the impact of various channel correlation models on MU-MIMO system performance, SINR, analytical asymptotic expressions of which are derived for analog MRC and digital MRC. We begin by deriving the expected signal and interference power, demonstrating that the signal-to-interference (SIR) ratio decreases with correlation when users have the same correlation matrices, while it increases when their correlation matrices are different. These finite system results are then extended by deriving asymptotic SINR expressions for both analog and digital MRC for benchmark scenarios of uncorrelated and fully correlated Rayleigh channels. Here, we once again demonstrate that the performance is critically dependent on the correlation scenario. We show that for uncorrelated fading the SINR converges to a constant. For fully correlated channels, and users with equal correlation matrices, SINR converges to zero, whereas for unequal 
correlation matrices SINR converges to zero or a constant, depending on the correlation model. New insights are provided, indicating that in certain circumstances, high correlation can improve system performance. The reason is explained via 3D surface plots based on exponential and one-ring correlation models.

\subsection{Introduction}

MRC in particular offers low computational complexity, while eliminating (in its digital form) interference by taking advantage of favourable propagation in large systems. An additional benefit of MRC is its suitability for distributed systems - the processing can be performed independently at each antenna cluster, without additional information exchange [7]. Hence, a study of digital and analog MRC is given in this chapter.

It is well known that due to practical constraints on array size, correlation will significantly impact the favourable propagation characteristics of massive MIMO systems [120]. The asymptotic behaviour of large systems with correlation (equal for all users) has been well studied (see $[7,27,34])$. Studies of correlation impact on linear precoding [121,122] demonstrate that correlation reduces system performance. This is in contrast to early work on SU MIMO-MRC $[123,124]$ which has shown that correlation can increase the system performance.

The above studies consider the classical Kronecker model, and assume equal correlation among users. Recently, the impact of correlation on system performance has been shown to be highly dependent on the correlation model. The authors of [125] contrast the Kronecker and Wichselberger models, where the former predicts a more severe performance degradation. In [119] it has been shown that the classical exponential correlation model 
gives a pessimistic performance prediction relative to that given by the onering model. Furthermore, [119] demonstrates that system heterogeneity, including correlation variability among users, enhances the MU system performance. Backed by measurement results demonstrating the variability in userspecific angular propagation parameters, [119] highlights the importance of considering such heterogeneity in system modeling.

In this chapter we provide new insights on the impact of correlation (including its heterogeneity) on the SINR and SE behaviour of digital MRC and the first such results for analog MRC. Specifically,

- We derive analytical expressions of SINRs for uplink analog MRC and digital MRC under different correlation scenarios when the number of antennas and users goes to infinity. The asymptotic behaviours of these cases are analysed. The findings suggest that the system performance is improved where there is perfect correlation within one user's channel but a random phase distribution among users. Thus, in some cases, correlation is beneficial and can help system performance. From the derived expression of SINR for i.i.d Rayleigh fading, around 21.5\% performance loss occurs with analog MRC compared with digital MRC. The system performance analysis under perfect correlation suggests that digital and analog MRC would have the same asymptotic behaviour.

- We derive a SIR expression based on an exponential correlation model, which takes both correlation coefficient and phase into consideration. The 3D surface plots based on this expression give great insight into why high correlations with random phases improve system performance. We also show that similar system behaviour occurs under a one-ring correlation model.

- The interference behaviour under perfect correlation with two special 
correlation models, exponential and one-ring are studied. We find that the asymptotic behaviour of interference mainly depends on the angular distribution of users.

\subsection{System Model}

We consider an uplink massive MIMO system with $N$ co-located antennas serving $K$ single antenna users. The $N \times 1$ channel vector for each user can be written as $\mathbf{h}_{i}=\mathbf{R}_{i}^{\frac{1}{2}} \mathbf{u}_{i}$, where $\mathbf{u}_{i} \sim \mathcal{C N}(0, \mathbf{I}), \mathbf{R}_{i}=\beta_{i} \boldsymbol{\Sigma}_{i}$ and $\boldsymbol{\Sigma}_{i}$ is the $N \times N$ spatial correlation matrix. $\beta_{i}$ is the large-scale link gain for the $i^{\text {th }}$ user. The fast-fading channel matrix can be written as $\mathbf{H}=\left[\mathbf{h}_{1} \mathbf{h}_{2} \ldots \mathbf{h}_{K}\right]$. We assume perfect channel knowledge at the BS and equal transmit power, $P_{\mathrm{t}}$, for each user. Thus, the received signal at the BS can be expressed as

$$
\mathbf{y}=\sqrt{P_{\mathrm{t}}} \mathbf{H} \mathbf{s}+\mathbf{n},
$$

where $\mathbf{n} \sim \mathcal{C N}\left(\mathbf{0}, \sigma_{\mathrm{n}}^{2}\right)$ is white Gaussian noise, $\mathbf{s}$ is the data symbol vector from the $K$ users and $\mathbb{E}\left[\mathbf{s s}^{H}\right]=\mathbf{I}$. Without loss of generality, $\sigma_{\mathrm{n}}^{2}$ is assumed to be 1 . The signal after combining at the BS to detect the $i^{\text {th }}$ user is given by

$$
\tilde{\mathbf{y}}_{i}=\sqrt{P_{\mathrm{t}}} \mathbf{g}_{i}^{\mathrm{H}} \mathbf{h}_{i} s_{i}+\sqrt{P_{\mathrm{t}}} \sum_{l=1, l \neq i}^{K} \mathbf{g}_{i}^{\mathrm{H}} \mathbf{h}_{l} s_{l}+\mathbf{g}_{i}^{\mathrm{H}} \mathbf{n},
$$

where $\mathbf{g}_{i}=\mathbf{h}_{i}$ for digital MRC and $\mathbf{g}_{i}=\hat{\mathbf{h}}_{i}$ for analog MRC, where $\hat{\mathbf{h}}_{i}=$ $\exp \left(j \angle \mathbf{h}_{i}\right)$ and $\angle \mathbf{h}_{i}$ indicates the vector of angles of $\mathbf{h}_{i}$. The corresponding SINR is given by

$$
\operatorname{SINR}_{i}=\frac{P_{\mathrm{t}}\left|\mathbf{g}_{i}^{\mathrm{H}} \mathbf{h}_{i}\right|^{2}}{P_{\mathrm{t}} \sum_{l=1, l \neq i}^{K}\left|\mathbf{g}_{i}^{\mathrm{H}} \mathbf{h}_{l}\right|^{2}+\mathbf{g}_{i}^{\mathrm{H}} \mathbf{g}_{i}} .
$$


For digital MRC, the mean signal and interference in (5.3) are given by

$$
\begin{gathered}
\mathbb{E}\left[P_{\mathrm{t}}\left|\mathbf{g}_{i}^{\mathrm{H}} \mathbf{h}_{i}\right|^{2}\right]=P_{t} \beta_{i}^{2}\left[\operatorname{tr}\left(\boldsymbol{\Sigma}_{i}^{2}\right)+N^{2}\right], \\
\mathbb{E}\left[P_{\mathrm{t}} \sum_{l \neq i}\left|\mathbf{g}_{i}^{\mathrm{H}} \mathbf{h}_{l}\right|^{2}\right]=P_{\mathrm{t}} \beta_{i} \sum_{l \neq i} \beta_{l} \operatorname{tr}\left(\boldsymbol{\Sigma}_{i} \boldsymbol{\Sigma}_{l}\right) .
\end{gathered}
$$

The relationship between correlation and performance is seen in (5.4) and (5.5) via the terms $\operatorname{tr}\left(\Sigma_{i}^{2}\right)$ and $\operatorname{tr}\left(\Sigma_{i} \Sigma_{l}\right)$. To explore this relationship, consider the exponential correlation model in [118] where $\left(\Sigma_{i}\right)_{r s}=\rho^{|s-r|} \exp \left(j(s-r) \phi_{i}\right)$ so that users have the same amplitude correlation parameter, $\rho$, but user specific phases, $\phi_{i}$ where $\phi_{i} \sim U[0,2 \pi]$. Using this model, some straightforward algebra allows $\operatorname{tr}\left(\Sigma_{i} \Sigma_{l}\right)$ to be written as

$$
\begin{aligned}
& \left\{2 \rho^{2 N+2}\left(\cos ((N+1) \Delta)-2 \rho^{2} \cos (N \Delta)+\rho^{4} \cos ((N-1) \Delta)\right)+N\left(1-\rho^{8}\right)\right. \\
& \left.+4 \rho^{4}+\cos (\Delta)\left((2 N-2) \rho^{6}-(2 N+2) \rho^{2}\right)\right\} /\left(1-2 \rho^{2} \cos (\Delta)+\rho^{4}\right)^{2},
\end{aligned}
$$

where $\Delta=\phi_{i}-\phi_{l}$. Obviously, $\operatorname{tr}\left(\Sigma_{i} \Sigma_{l}\right)$ is a function of both $\rho$ and $\Delta$, but what is interesting is that the relationship between interference and $\rho$ is different for different values of $\Delta$. To see this, consider two special cases of (5.6)

$$
\begin{array}{cc}
\operatorname{tr}\left(\boldsymbol{\Sigma}_{i} \boldsymbol{\Sigma}_{l}\right)=\frac{2 \rho^{2 N+2}+N\left(1-\rho^{2}\right)\left(1+\rho^{2}\right)-2 \rho^{2}}{\left(1-\rho^{2}\right)^{2}}, & \Delta=0, \\
\operatorname{tr}\left(\boldsymbol{\Sigma}_{i} \boldsymbol{\Sigma}_{l}\right)=\frac{N+4 \rho^{4}-N \rho^{8}-4(-1)^{N / 2} \rho^{2 N+4}}{\left(1+\rho^{4}\right)^{2}}, & \Delta=\pi / 2,
\end{array}
$$

where (5.8) is for the case of even $N$. When the phase parameters are aligned $(\Delta=0)$, the two correlation matrices are identical and (5.7) shows that the interference grows with $\rho$. When the two phases are orthogonal $(\Delta=\pi / 2)$, (5.8) shows that the interference decreases with $\rho$. Hence, increasing the size of the correlation can have opposite effects when users experience the same 
correlation matrix $\left(\phi_{i}=\phi_{l}\right)$ and when the correlation matrices differ $\left(\phi_{i}-\phi_{l}=\right.$ $\pi / 2$ ). Equivalent results to (5.4) and (5.5) for analog MRC are available in [126] but are given in terms of Gaussian hypergeometric functions which make further analysis difficult. Hence, we look at the extreme cases of zero and perfect correlation in Sec. 5.3 below.

\subsection{Asymptotic SINR Analysis}

We derive the asymptotic SINR for analog and digital MRC under two benchmark scenarios of i.i.d. and perfectly correlated Rayleigh fading. We assume $N$ and $K$ grow at the same rate, $\alpha=\frac{N}{K}$ is fixed. The derivations require the strong law of large numbers, a suitable version of which follows.

Result 1. If $X_{1}, X_{2}, \ldots$ are independent with finite means $\mu_{1}, \mu_{2}, \ldots$ and variances $\sigma_{1}^{2}, \sigma_{2}^{2}, \ldots$, then $\frac{1}{L} \sum_{n=1}^{L} X_{n} \stackrel{\text { as }}{\rightarrow} \mu$, as $L \rightarrow \infty$, where $\mu=\lim _{L \rightarrow \infty}\left(\frac{1}{L} \sum_{n=1}^{L} \mu_{n}\right)$ if $\sum_{n=1}^{L} \frac{1}{L^{2}} \operatorname{Var}\left(X_{n}\right)<\infty[127$, Theorem 5.4 .3$]$, where $\stackrel{a s}{\rightarrow}$ denotes almost sure convergence [128, p.405]. Almost sure convergence is defined as a property of a sequence of random variables, $X_{1}, X_{2}, X_{3}, \cdots, X_{n}$, where $X_{n}$ converges almost surely to a random variable, $X$, shown by $X_{n} \stackrel{a s}{\rightarrow} X$, if $P\left(\left\{s \in S: \lim _{n \rightarrow \infty} X_{n}(s)=X(s)\right\}\right)=1$. In this notation, $s$ denotes an element in the sample space and $S$ is the entire sample space. 


\subsubsection{Asymptotic analysis for i.i.d. Rayleigh fading}

First, for digital MRC, from (5.3), the interference power is,

$$
\begin{aligned}
& P_{\mathrm{t}} \sum_{\substack{l=1 \\
l \neq i}}^{K}\left|\mathbf{h}_{i}^{\mathrm{H}} \mathbf{h}_{l}\right|^{2}=P_{\mathrm{t}} \sum_{\substack{l=1 \\
l \neq i}}^{K} \mathbf{h}_{l}^{\mathrm{H}} \mathbf{h}_{i} \mathbf{h}_{i}^{\mathrm{H}} \mathbf{h}_{l} \\
& =P_{\mathrm{t}} \sum_{\substack{l=1 \\
l \neq i}}^{K} \mathbf{h}_{l}^{\mathrm{H}} \Phi\left(\begin{array}{lll}
\mathbf{h}_{i}^{\mathrm{H}} \mathbf{h}_{i} & & \\
& \ddots & \\
& & 0
\end{array}\right) \Phi \mathbf{h}_{l} \\
& \equiv P_{\mathrm{t}} \sum_{\substack{l=1 \\
l \neq i}}^{K} \beta_{i} \beta_{l}\left|u_{l 1}\right|^{2} \mathbf{u}_{i}^{\mathrm{H}} \mathbf{u}_{i},
\end{aligned}
$$

where $\Phi$ is unity matrix and $\equiv$ denotes statistical equivalence. We say that a random variable, $X$, is statistically equivalent to another random variable, $Y$, if the distributions of $X$ and $Y$ are the same. Any i.i.d Gaussian vector multiplied by a unity matrix is statistically equivalent to the original random vector. In (5.9), $u_{l 1}$ is the first element of $\mathbf{u}_{l}$ and the second equality follows from the rank-1 eigen-decomposition of $\mathbf{h}_{i} \mathbf{h}_{i}^{\mathrm{H}}$. The desired signal power is $P_{\mathrm{t}}\left(\mathbf{h}_{i}^{\mathrm{H}} \mathbf{h}_{i}\right)^{2}=P_{\mathrm{t}} \beta_{i}^{2}\left(\mathbf{u}_{i}^{\mathrm{H}} \mathbf{u}_{i}\right)^{2}$ and the noise power is $\mathbf{h}_{i}^{\mathrm{H}} \mathbf{h}_{i}=\beta_{i} \mathbf{u}_{i}^{\mathrm{H}} \mathbf{u}_{i}$. Substituting these powers into (5.3) and simplifying we obtain

$$
\operatorname{SINR}_{i}^{\mathrm{D}}=\frac{P_{\mathrm{t}} \beta_{i} \mathbf{u}_{i}^{\mathrm{H}} \mathbf{u}_{i} / N}{\frac{P_{\mathrm{t}}(K-1)}{N} \sum_{\substack{l=1 \\ l \neq 1}}^{K} \frac{\beta_{l}\left|u_{l 1}\right|^{2}}{K-1}+\frac{1}{N}},
$$

where D denotes digital MRC. Using Result 1, the numerator and denominator of (5.10) both converge almost surely, giving

$$
\lim _{N \rightarrow \infty} \operatorname{SINR}_{i}^{\mathrm{D}}=\frac{\beta_{i}}{\bar{\beta}} \alpha,
$$


where $\bar{\beta}=\lim _{N \rightarrow \infty} \sum_{i=1}^{K} \beta_{i} / K$ is the asymptotic mean of the link gains. For analog MRC, similar steps lead to the result

$$
\operatorname{SINR}_{i}^{\mathrm{A}}=\frac{P_{\mathrm{t}} \beta_{i}\left|\hat{\mathbf{u}}_{i}^{\mathrm{H}} \mathbf{u}_{i} / N\right|^{2}}{\frac{P_{\mathrm{t}}(K-1)}{K-1} \sum_{\substack{l=1 \\ l \neq i}}^{K} \beta_{l}\left|\frac{\hat{\mathbf{u}}_{i}^{\mathrm{H}} \mathbf{u}_{l}}{N}\right|^{2}+\frac{1}{N}}
$$

where $\hat{\mathbf{h}}_{i}=\sqrt{\beta_{i}} \hat{\mathbf{u}}_{i}$. For the numerator, from Result $\mathbf{1}$ we have ${ }^{1}$

$$
\frac{\hat{\mathbf{u}}_{i}^{\mathrm{H}} \mathbf{u}_{i}}{N}=\sum_{r=1}^{N} \frac{\left|u_{i r}\right|}{N} \stackrel{\text { as }}{\rightarrow} \mathbb{E}\left(\left|u_{i r}\right|\right)=\int_{0}^{\infty}\left|u_{i r}\right| e^{-\left|u_{i r}\right|^{2}} d_{\left|u_{i r}\right|^{2}}=\Gamma\left(\frac{3}{2}\right)=\frac{\sqrt{\pi}}{2}
$$

For the interference term, we note that

$$
\sum_{l=1, l \neq i}^{K} \beta_{l}\left|\hat{\mathbf{u}}_{i}^{\mathrm{H}} \mathbf{u}_{l}\right|^{2} \equiv \sum_{l=1, l \neq i}^{K} \beta_{l}\left|u_{l 1}\right|^{2} N
$$

which follows from the rank-1 eigen-decomposition of $\hat{\mathbf{u}}_{i} \hat{\mathbf{u}}_{i}^{\mathrm{H}}$. Thus, from $\mathbf{R e}-$ sult 1 , the interference term in (??) is

$$
P_{\mathrm{t}} \frac{K-1}{N} \sum_{l=1, l \neq i}^{K} \beta_{l} \frac{\left|u_{l 1}\right|^{2}}{(K-1)} \stackrel{\text { as }}{\rightarrow} P_{\mathrm{t}} \frac{\bar{\beta}}{\alpha}
$$

Substituting (5.13) and (5.15) into (??), we obtain

$$
\lim _{N, K \rightarrow \infty} \operatorname{SINR}_{i}^{\mathrm{A}}=\frac{\pi \beta_{i}}{4 \bar{\beta}} \alpha
$$

Compared with digital MRC, analog MRC suffers a $\left(1-\frac{\pi}{4}\right) \times 100 \approx 21.5 \%$ performance loss in the asymptotic SINR.

$$
{ }^{1} \Gamma(n)=\int_{0}^{\infty} x^{n-1} e^{-x} d x
$$




\subsubsection{Asymptotic analysis, perfect correlation, equal matrices}

Consider the perfectly correlated channel where $\mathbf{R}_{i}=\beta_{i} \boldsymbol{\Sigma}_{i}$ and all the elements of $\boldsymbol{\Sigma}_{i}$ equal one. Here, $\mathbf{h}_{i}=\mathrm{h}_{i 1}[11 \ldots 1]^{T}$ and $\hat{\mathbf{h}}_{i}=\exp \left(j \angle \mathrm{h}_{i 1}\right)[11 \ldots 1]^{T}$. Substituting the perfectly correlated channels and associated combiners into (5.3) gives:

$$
\operatorname{SINR}_{i}^{\mathrm{D}}=\operatorname{SINR}_{i}^{\mathrm{A}}=\frac{\beta_{i}\left|u_{i 1}\right|^{2}}{\sum_{l=1, l \neq i}^{K} \beta_{l}\left|u_{l 1}\right|^{2}+1 / P_{\mathrm{t}} N} .
$$

Dividing the numerator and denominator by $K$ and using Result 1 gives

$$
\operatorname{SINR}_{i}^{\mathrm{A}}=\operatorname{SINR}_{i}^{\mathrm{D}} \stackrel{a s}{\rightarrow} 0
$$

\subsubsection{Asymptotic analysis, perfect correlation, unequal matri- ces}

Here we investigate two types of correlation structures for uniform linear arrays. The exponential ${ }^{2}$ correlation model in [118] has a user specific phase, $\phi \sim U[0,2 \pi]$, for the correlation parameter and for perfect correlation (amplitude 1), the correlation matrix is defined by $\left(\Sigma_{i}\right)_{r s}=\exp \left(j(s-r) \phi_{i}\right)$. The second model is the classic one-ring model [57]. For this model, taking the limit as the angle spread vanishes gives a perfectly correlated correlation matrix also defined by $\left(\Sigma_{i}\right)_{r s}=\exp \left(j(s-r) \phi_{i}\right)$ but here, $\phi_{i}=2 \pi d \sin \left(\theta_{i}\right)$ where $d$ is the antenna spacing and $\theta_{i}$ is the angle of departure for user $i$. For both models, defining $\mathbf{a}_{i}=\left[1 \exp \left(-j \phi_{i}\right) \exp \left(-j 2 \phi_{i}\right) \ldots \exp \left(-j(N-1) \phi_{i}\right)\right]^{T}$ allows the channel vectors to be written as $\mathbf{h}_{i}=\mathrm{h}_{i 1} \mathbf{a}_{i}$. Following the steps in Sec.

\footnotetext{
${ }^{2}$ Here, exponential refers to a correlation structure that has an exponential decay in amplitude. Here, with perfect correlation, the amplitude is one so that the decay is not obvious.
} 
5.3.2 the corresponding SINRs are

$$
\operatorname{SINR}_{i}^{\mathrm{A}}=\operatorname{SINR}_{i}^{\mathrm{D}}=\frac{\left|h_{i 1}\right|^{2}}{\sum_{\substack{l=1 \\ l \neq i}}^{K}\left|h_{l 1}\right|^{2}\left|\frac{\mathbf{a}_{i}^{H} \mathbf{a}_{l}}{N}\right|^{2}+\frac{1}{P_{\mathrm{t}} N}}
$$

From (5.19), we see that the SINR depends on the limiting behaviour of the interference component denoted by $I$. We explore this limiting behaviour by deriving $\mathbb{E}(I)$ :

$$
\begin{aligned}
\mathbb{E}(I) & =\frac{K-1}{N} \mathbb{E}\left(\left|h_{l 1}\right|^{2}\right) \frac{1}{N} \mathbb{E}\left[\left|\mathbf{a}_{i}^{H} \mathbf{a}_{l}\right|^{2}\right] \\
& \rightarrow \mu_{I}^{\infty}=\frac{1}{\alpha} \lim _{N \rightarrow \infty} \frac{1}{N} \mathbb{E}\left[\left|\mathbf{a}_{i}^{H} \mathbf{a}_{l}\right|^{2}\right] \\
& =\frac{1}{\alpha} \lim _{N \rightarrow \infty} \frac{1}{N} \sum_{h=0}^{N-1} \sum_{k=0}^{N-1} \mathbb{E}\left[e^{j(h-k) \phi_{i}}\right] \mathbb{E}\left[e^{-j(h-k) \phi_{l}}\right] \\
& =\frac{1}{\alpha} \lim _{N \rightarrow \infty} \frac{1}{N} \sum_{r=-(N-1)}^{N-1}(N-r)\left|\mathbb{E}\left[e^{j r \phi_{i}}\right]\right|^{2} \\
& =\frac{1}{\alpha} \lim _{N \rightarrow \infty}\left\{1+2 \sum_{r=1}^{N-1}\left(1-\frac{r}{N}\right)\left|\mathbb{E}\left[e^{j r \phi_{i}}\right]\right|^{2}\right\}
\end{aligned}
$$

Next, we derive $\left|\mathbb{E}\left[e^{j r \phi_{i}}\right]\right|$ for the two correlation models.

\section{Exponential Correlation}

The model in [118] has $\phi_{i} \sim U[0,2 \pi]$ for which $\mathbb{E}\left[e^{j r \phi_{i}}\right]=0$ and $\mathbb{E}(I) \rightarrow \frac{1}{\alpha}$. For the more general case where $\phi_{i} \sim U[a, b]$ we have

$$
\begin{aligned}
\left|\mathbb{E}\left[e^{j r \phi_{i}}\right]\right| & =\left|(b-a)^{-1} \int_{a}^{b} e^{j r \phi_{i}} d \phi_{i}\right|=\frac{2|\sin [0.5 r(b-a)]|}{r(b-a)} \\
& \leq \frac{2}{r(b-a)} .
\end{aligned}
$$


Substituting (5.21) into (5.20) we have

$$
\begin{aligned}
\mu_{I}^{\infty} & \leq \frac{1}{\alpha}\left\{1+\frac{8}{(b-a)^{2}} \lim _{N \rightarrow \infty}\left\{\sum_{r=1}^{N-1} \frac{1}{r^{2}}-\frac{1}{N} \sum_{r=1}^{N-1} \frac{1}{r}\right\}\right\} \\
& \leq \frac{1}{\alpha}\left\{1+\frac{4 \pi^{2}}{3(b-a)^{2}}\right\}
\end{aligned}
$$

since $\sum_{r=1}^{N-1} r^{-2} \rightarrow \pi^{2} / 6$ and $\sum_{r=1}^{N-1}(N r)^{-1} \rightarrow 0$. Hence, for all uniform distributions, the mean interference is finite.

\section{One-ring Correlation}

Here, we have $\phi_{i}=2 \pi d \sin \theta_{i}$, where $\theta_{i} \sim U[0,2 \pi]$. Hence,

$$
\mathbb{E}\left[e^{j r \phi_{i}}\right]=\frac{1}{2 \pi} \int_{0}^{2 \pi} e^{j r 2 \pi d \sin \left(\theta_{i}\right)} d \theta_{i}=J_{0}(2 \pi d r)
$$

Substituting (5.22) into (5.20) we have $\mu_{I}^{\infty}=\frac{1}{\alpha}(1+2 S)$, where

$$
S=\lim _{N \rightarrow \infty}\left\{\sum_{r=1}^{N-1}\left(1-\frac{r}{N}\right)\left[J_{0}(2 \pi d r)\right]^{2}\right\} .
$$

From [129, Eq. 10.17.3], for large arguments ${ }^{3} J_{0}(z)=\sqrt{2 / \pi z} \cos (z-\pi / 4)+$ $\mathcal{O}\left(z^{-3 / 2}\right)$. Hence, $S$ exists (is finite) if and only if $S_{1}$ exists where

$$
\begin{aligned}
S_{1} & =\lim _{N \rightarrow \infty} \frac{1}{\pi^{2} d} \sum_{r=1}^{N-1}\left(\frac{1}{r}-\frac{1}{N}\right) \cos ^{2}\left(2 \pi r d-\frac{\pi}{4}\right) \\
& \geq \lim _{N \rightarrow \infty} \frac{1}{\pi^{2} d} \sum_{r=1}^{N-1} \frac{1}{r} \cos ^{2}\left(2 \pi r d-\frac{\pi}{4}\right)-\frac{1}{\pi^{2} d} .
\end{aligned}
$$

\footnotetext{
${ }^{3}$ Note that the sum in (5.23) will always be finite for a finite number of terms. The only thing that can make it explode is the behaviour of the later terms when $r$ is large. This means we are interested in large $r$ which means a large argument as the argument is $2 \pi d r$.
} 
Using the double angle formula, the first summation in (5.24) can be written as

$$
\sum_{r=1}^{N-1} \frac{1}{r} \cos ^{2}\left(2 \pi r d-\frac{\pi}{4}\right)=\frac{1}{2} \sum_{r=1}^{N-1} \frac{1}{r}+\frac{1}{2} \sum_{r=1}^{N-1} \frac{1}{r} \sin (4 \pi r d) .
$$

Note that $\sum_{r=1}^{N-1} \frac{1}{r}-\log (N-1) \rightarrow C$, where $C$ is the Euler constant [130, p. 3]. Hence, the asymptotic behaviour of $\sum_{r=1}^{N-1} \frac{1}{r}$ is logarithmic in $N$. From [131, p. 43], the second sum in (5.25) is finite,

$$
\frac{1}{2} \sum_{r=1}^{N-1} \frac{1}{r} \sin (4 \pi r d)=\frac{\pi}{2}-2 \pi d \quad(\bmod 2 \pi)
$$

Since the first term in 5.25 is logarithmic in $N$ and the second term is finite, we see that the growth of $S_{1}$ is also logarithmic in $N . \quad \frac{1}{2} \sum_{r=1}^{N-1} \frac{1}{r}$ diverges logarithmically. Thus, $S_{1}$ diverges, causing both $S$ and $\mu_{I}^{\infty}$ to diverge. The simulation results in Sec. 5.4 also support this claim.

\subsubsection{Analog MRC correlation analysis}

Next, we consider analog MRC and repeat some of the results from Chapter 4 for the co-located scenario considered here. The expected desired signal power after analog MRC processing [126] is,

$$
\mathbb{E}\left\{\left|\hat{\mathbf{h}}_{i}^{H} \mathbf{h}_{i}\right|^{2}\right\}=N_{\mathrm{t}} \beta_{i}+\beta_{i} \sum_{\substack{j=1 \\ j \neq k}}^{N_{\mathrm{t}}} \sum_{\substack{k=1 \\ j \neq k}}^{N_{\mathrm{t}}} \delta_{i j k}
$$

where 


$$
\delta_{i j k}=\frac{\pi}{4}\left(1-\left|\rho_{i j k}\right|^{2}\right)_{2}^{2} F_{1}\left(\frac{3}{2}, \frac{3}{2}, 1 ;\left|\rho_{i j k}\right|^{2}\right)
$$

and $\rho_{i j k}$ is the $(j k)^{\text {th }}$ element of $\Sigma_{i}$. The expected total interference power after analog MRC processing is,

$$
\mathbb{E}\left\{\left|\hat{\mathbf{h}}_{i}^{H} \mathbf{h}_{l}\right|^{2}\right\}=N_{\mathrm{t}} \beta_{l}+\sum_{\substack{j=1 \\ j \neq k}}^{N_{\mathrm{t}}} \sum_{\substack{k=1 \\ N_{\mathrm{t}}}}\left(\mathbf{R}_{l}\right)_{k j} \kappa_{i j k}
$$

where

$$
\kappa_{i j k}=\frac{\pi}{4} \rho_{i j k}\left(1-\left|\rho_{i j k}\right|^{2}\right)_{2} F_{1}\left(\frac{3}{2}, \frac{3}{2}, 2 ;\left|\rho_{i j k}\right|^{2}\right) .
$$

From [132, Eq.15.3.3, p. 559], we have

$$
{ }_{2} F_{1}(\alpha, \beta, \gamma ; Z)=(1-Z)^{\gamma-\alpha-\beta}{ }_{2} F_{1}(\gamma-\alpha, \gamma-\beta, \gamma ; Z)
$$

Hence, $\delta_{i j k}$ and $\kappa_{i j k}$ can be rewritten as $\delta_{i j k}=(\pi / 4)_{2} F_{1}\left(-\frac{1}{2},-\frac{1}{2}, 1 ;\left|\rho_{i j k}\right|^{2}\right)$ and $\kappa_{i j k}=\left(\frac{\pi}{4} \rho_{i j k}\right){ }_{2} F_{1}\left(\frac{1}{2}, \frac{1}{2}, 2 ;\left|\rho_{i j k}\right|^{2}\right)$. Substituting these expressions in (5.27) and (5.29) gives

$$
\mathbb{E}\left\{\left|\hat{\mathbf{h}}_{i}^{H} \mathbf{h}_{i}\right|^{2}\right\}=N_{\mathrm{t}} \beta_{i}+\frac{\pi}{4} \beta_{i} \sum_{\substack{j=1 \\ j \neq k}}^{N_{\mathrm{t}}} \sum_{\substack{k=1 \\ j \neq k}}^{N_{\mathrm{t}}} F_{1}\left(-\frac{1}{2},-\frac{1}{2}, 1 ;\left|\rho_{i j k}\right|^{2}\right),
$$


and

$$
\mathbb{E}\left\{\left|\hat{\mathbf{h}}_{i}^{H} \mathbf{h}_{l}\right|^{2}\right\}=N_{\mathrm{t}} \beta_{l}+\frac{\pi}{4} \beta_{l} \sum_{\substack{j=1 \\ N_{\mathrm{t}}}}^{N_{k=1}} \sum_{\substack{\mathrm{t} \\ j=1}}\left(\rho_{l k j} \rho_{i j k}\right)_{2} F_{1}\left(\frac{1}{2}, \frac{1}{2}, 2 ;\left|\rho_{i j k}\right|^{2}\right) .
$$

Now, using known results on hypergeometric functions, ${ }_{2} F_{1}\left(-\frac{1}{2},-\frac{1}{2}, 1 ; Z\right)$ and ${ }_{2} F_{1}\left(\frac{1}{2}, \frac{1}{2}, 2 ; Z\right)$ are monotonic increasing in $Z$ [132, Eq.15.2.1, p. 557], ${ }_{2} F_{1}\left(-\frac{1}{2},-\frac{1}{2}, 1 ; 0\right)={ }_{2} F_{1}\left(\frac{1}{2}, \frac{1}{2}, 2 ; 0\right)=1[132$, Eq.15.1.1, p. 556] and ${ }_{2} F_{1}\left(-\frac{1}{2},-\frac{1}{2}, 1 ; 1\right)={ }_{2} F_{1}\left(\frac{1}{2}, \frac{1}{2}, 2 ; 1\right)=4 / \pi\left[132\right.$, Eq.15.1.20, p. 556]. Hence, $\delta_{i j k}$ increases smoothly from $\pi / 4$ to 1 as $\left|\rho_{i j k}\right|$ increases form 0 to 1 . Similarly, $\kappa_{i j k}$ increases smoothly from 0 to 1 as $\left|\rho_{i j k}\right|$ increases form 0 to 1 . This makes (5.32) and (5.33) easier to interpret. We see that the mean signal power in (5.32) is an increasing function of $\left|\rho_{i j k}\right|$. The interference behaviour depends on the similarity of the correlation matrices. If $\Sigma_{i}=\Sigma_{l}$, then $\rho_{l k j} \rho_{i j k}=\left|\rho_{i j k}\right|^{2}>0$ and interference also grows with $\left|\rho_{i j k}\right|$. However, if $\Sigma_{i} \neq \Sigma_{l}$, then $\rho_{l k j} \rho_{i j k}$ is a complex constant and the double summation in (5.33) will not necessarily grow with $\left|\rho_{i j k}\right|$ as terms may cancel. Again, we are seeing the important property where correlation is detrimental with equal correlation across users but correlation can be beneficial with sufficient variation across users.

\subsection{Numerical Results}

The limiting results in Sec. 5.3 such as (5.11) and (5.16) depend on the link gains, $\beta_{i}$. Hence, in order to demonstrate convergence issues, we do not use the classic path-loss, log-normal shadowing models because the large variation will mask the limiting effects. We adopt a link gain model similar to that in [27] to counter this problem. We also consider two scenarios for $\beta_{i}$ : equal and unequal for each user. The equal link gain case serves as a reference and here we assume $\beta_{i}=1$ for all users. For the unequal case, we set $\beta_{i}=1$ for 
the desired user and generate $\beta_{l}$ for the interfering users from the exponential decay function $A e^{-\lambda z_{l}}$ with a mean value of $\frac{1}{K-1} \sum_{l \neq i} \beta_{l}=1$. We guarantee a minimum link gain by truncating the exponential decay so that the weakest user is $10 \%$ of the strongest. The resulting model is $\beta_{l}=A \exp \left(-\frac{(l-1) \lambda}{(K-1)}\right)$ where $\lambda=-\log (0.1)$ which gives $K-1$ evenly spread values of $\beta_{l}$ following an exponential decay over $\left[\frac{1}{10} A, A\right]$.

In Fig. 5.1, we present simulation results of the mean SINR vs $N$ for analog and digital MRC in i.i.d. Rayleigh fading with $P_{\mathrm{t}}=1, \alpha=2$ and as discussed above, $\bar{\beta}=\beta_{i}=1$. With these parameters (5.11) and (5.16) give the limits 2 and $\pi / 2$ which are verified in the figure. The unequal power case converges slightly more slowly as the power variation gives less averaging and stability compared to the equal power case. Also in Fig. 5.1 we give equivalent results

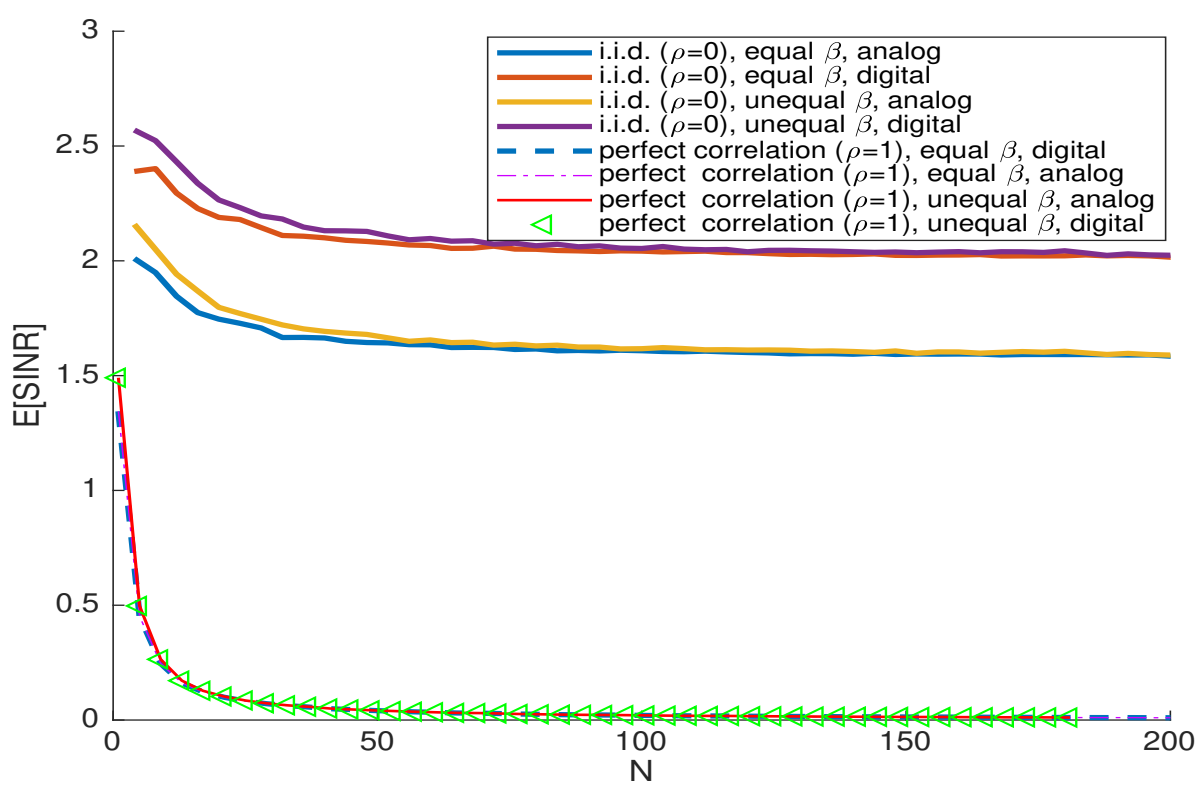

Figure 5.1: $\mathbb{E}[$ SINR] vs $N$ for analog and digital MRC; i.i.d. and perfect correlation, equal and unequal link gains.

with the same parameters for the case of perfect correlation with equal correlation matrices considered in Sec. 5.3.2. As shown in (5.18), the limiting SINR 
is zero for both analog and digital MRC. In Fig. 5.2 similar results are shown for both SINR and interference with perfect correlation and unequal correlation matrices based on the exponential correlation structure considered in Sec. 5.3.3. The parameters, $P_{\mathrm{t}}=50 \mathrm{~dB}, \alpha=2$, and $\phi_{i} \sim U[0,2 \pi]$ are used and equal link gains are assumed. The analysis predicts a finite limiting interference, $\mathbb{E}(I) \rightarrow \frac{1}{\alpha}=\frac{1}{2}$ and a corresponding finite and non-zero SINR. Both results are verified in Fig. 5.2.
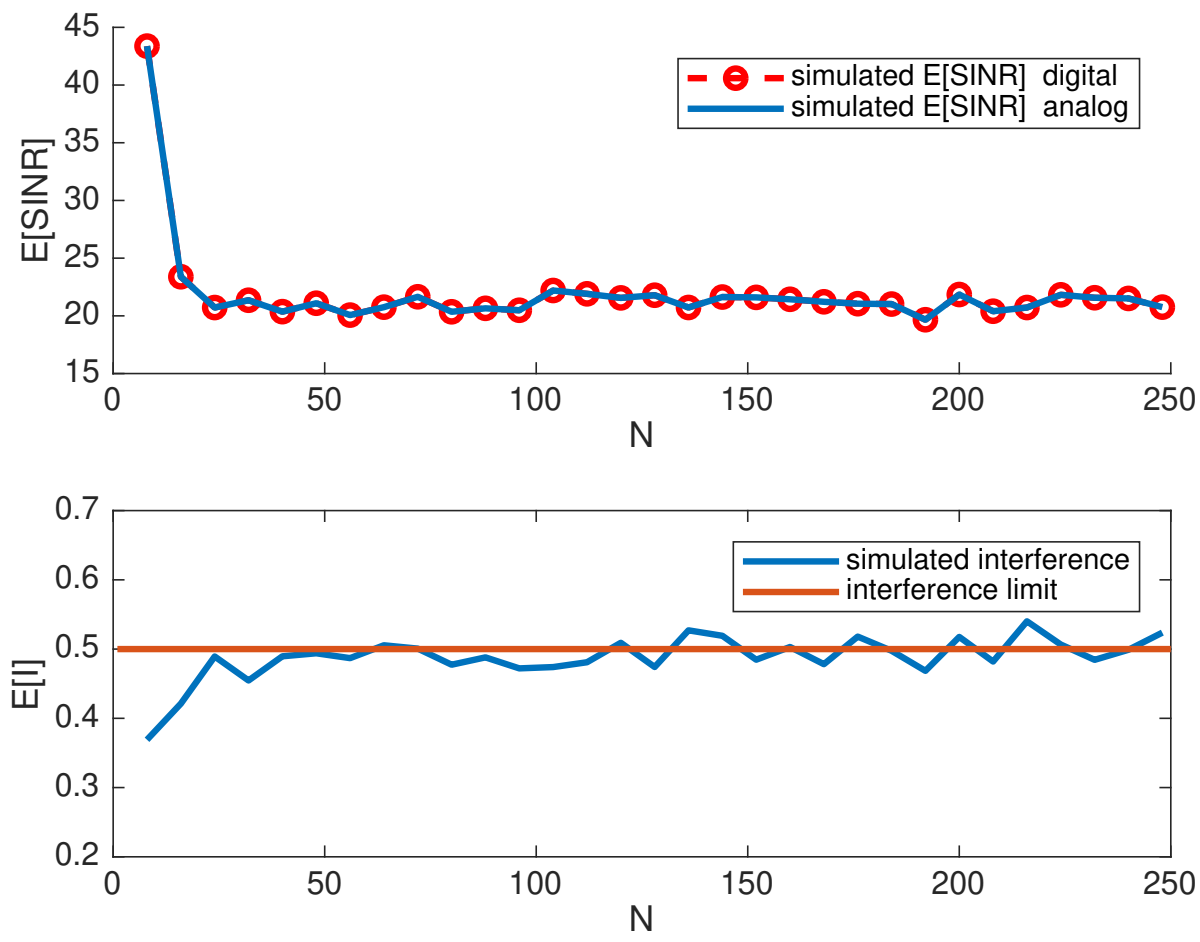

Figure 5.2: $\mathbb{E}[S I N R]$ and $\mathbb{E}[I]$ vs $N$; perfect correlation, exponential model, unequal correlation matrices.

In Fig. $5.3^{4}$ we show the divergence of the interference with perfect correlation and unequal correlation matrices based on the one-ring model in

\footnotetext{
${ }^{4}$ The top figure is plotted against $N$ and the logarithmic pattern is hard to see. Hence, the results are shown again in the lower figure on a log scale where a linear plot shows logarithmic behaviour. The reason for plotting the derived results and not simulations is that the scale goes up to a very large numbers of antennas for which simulation is problematic.
} 
Sec. 5.3.3. Equal link gains and user specific angles, $\theta_{i} \sim U[0,2 \pi]$, are assumed. In the upper figure we see that the derived interference (obtained by substituting (5.22) into (5.20)) agrees well with the simulated interference. The slow growth of interference with $N$ is clear here and this is made precise in the lower figure which demonstrates the logarithmic relationship predicted in the analysis (see (5.25)).
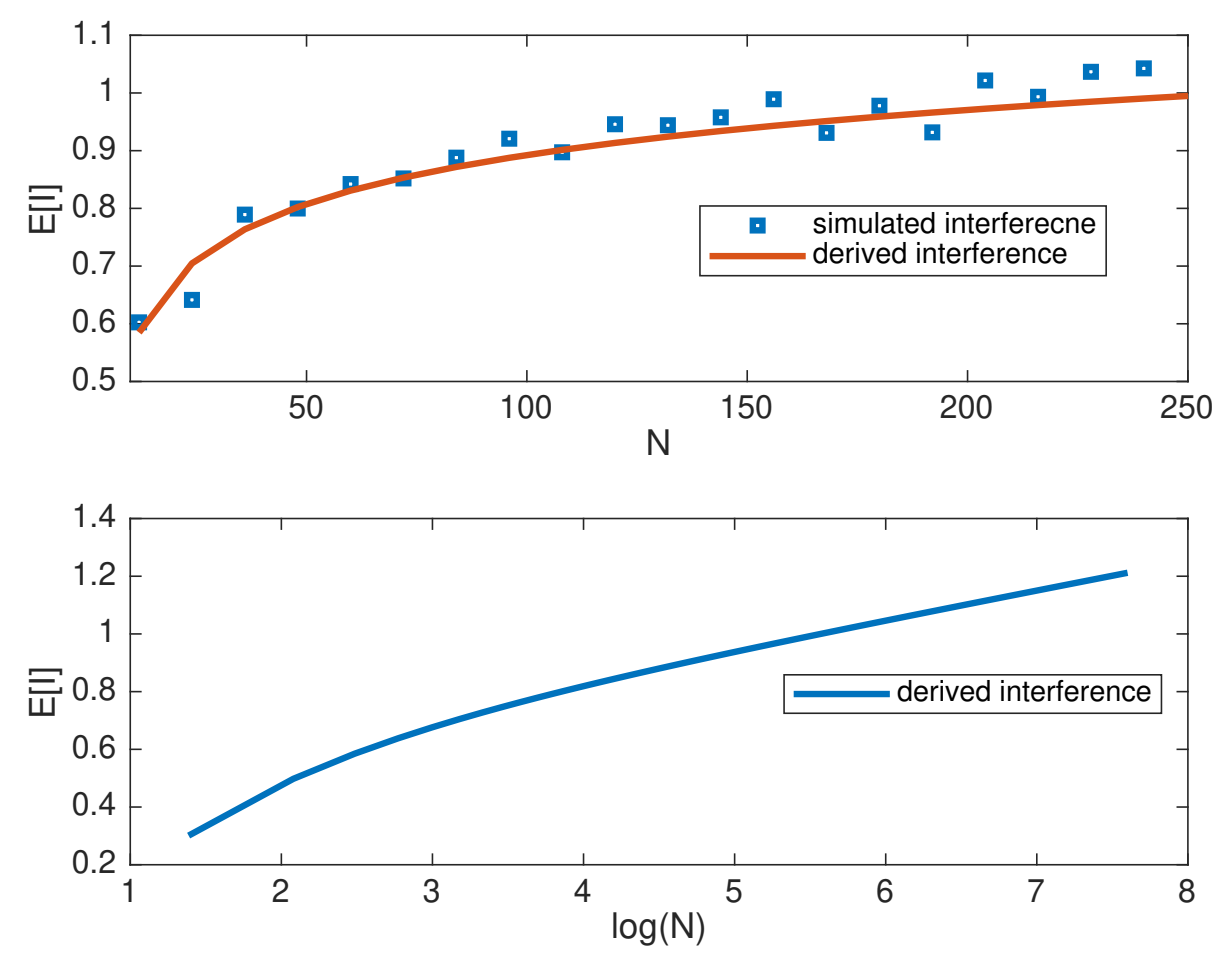

Figure 5.3: $\mathbb{E}[I]$ vs $N$ and $\log (N)$ for perfectly correlated Rayleigh fading with unequal correlation matrices (one-ring model).

Next, we consider general levels of correlation between the benchmark results of i.i.d. and perfectly correlated channels. From (5.4) and (5.5), we see that $T_{i i}=\operatorname{tr}\left(\Sigma_{i}^{2}\right)$ and $T_{i l}=\operatorname{tr}\left(\Sigma_{i} \Sigma_{l}\right)$ control the effect of correlation on signal power and interference. For the exponential correlation model, $T_{i i}$ and $T_{i l}$ are given by (5.6) which is a function of $\rho, N$ and $\Delta=\phi_{i}-\phi_{l}$. Hence, in Fig. 5.4 and Fig. 5.5 we plot $T_{i l}$ and $T_{i i} / T_{i l}$ against $\rho$ and $\Delta$ for $N=32$. The first mea- 
sures interference while the second gives the size of the signal term relative to interference. The interaction between the amplitude of the correlation parameter $(\rho)$ and the difference in the phases of the correlation parameters $(\Delta)$ is clear. Large amplitude correlation helps the signal relative to interference, thus enhancing SINR, unless the phases are very similar $(\Delta \approx 0$ or $\Delta \approx 2 \pi)$ when the SINR is adversely affected by large interference. Hence, as long as there is diversity in the phase parameters, increasing correlation is beneficial to performance. The same trends are observed for the one-ring model where reducing the angle spread (corresponding to increased correlation) is beneficial as long as there is diversity in the central angles of the users.

In Fig. 5.6 we show the simulated spectral efficiency CDFs for analog MRC assuming the exponential correlation structure. The system model includes path loss and lognormal shadowing effects. The link gains are given by $\beta_{i}=A \zeta_{i}\left(d_{0} / d_{i}\right)^{\gamma}$, where $d_{i}$ is the distance to the BS and $\zeta_{i}$ is lognormal shadowing. There are four users each with a single antenna uniformly located in a circular cell with the radius of 100 meters. The unit-less constant is $A=30 \mathrm{~dB}$, the reference distance is $d_{0}=1$ meter, $N_{\mathrm{t}}=32$, the pathloss exponent is $\gamma=3.5$ and the standard deviation of shadowing is $6 \mathrm{~dB}$. The transmit power $P_{\mathrm{t}}$ is chosen to guarantee that $95 \%$ of the time the SNR exceeds $0 \mathrm{~dB}$. As predicted by the analysis, for a fixed correlation parameter (equal correlation matrices for the users) increasing the correlation decreases spectral efficiency whereas for differing correlation parameters (unequal correlation matrices for the users), correlation improves the spectral efficiency.

\subsection{Conclusion}

We have presented the first analysis of the effects of correlation on analog processing, compared analog to digital MRC and demonstrated that hetero- 


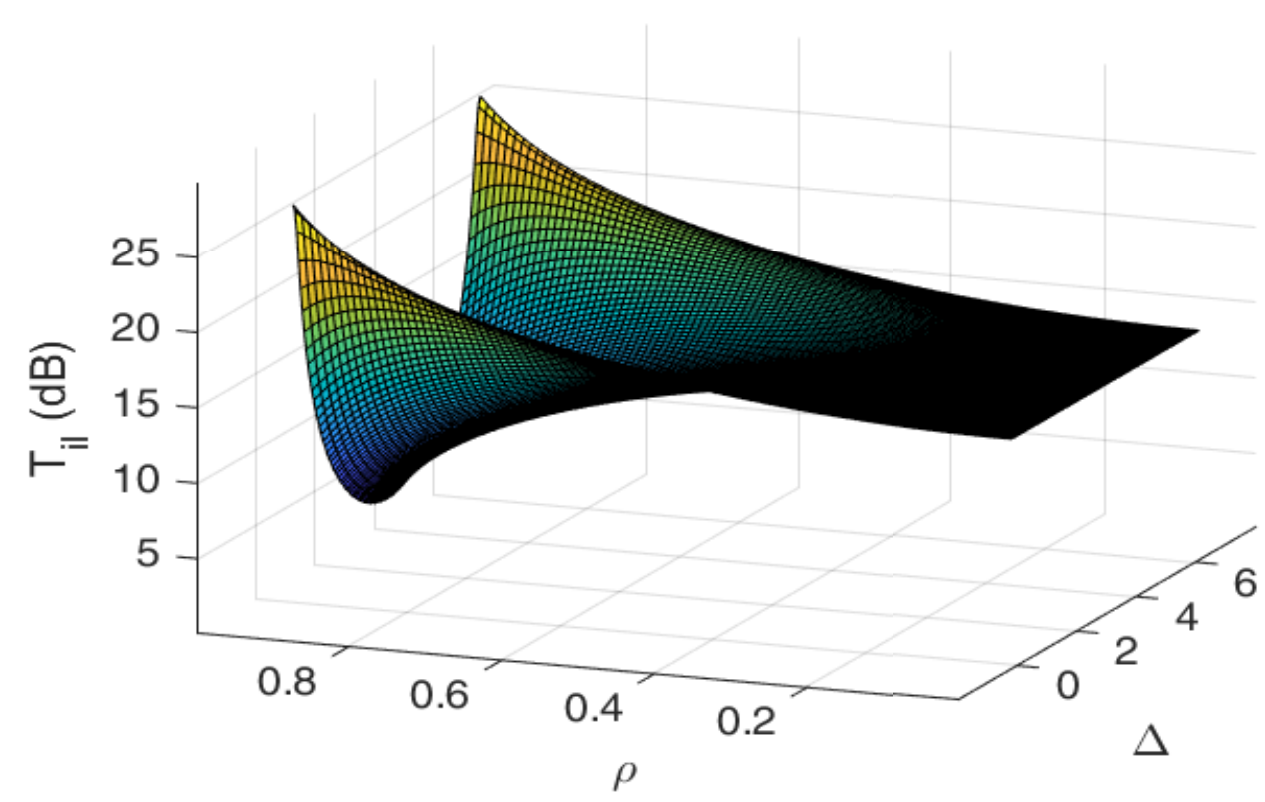

Figure 5.4: $T_{i l}(\mathrm{~dB})$ vs $\rho$ and $\Delta ; N=32$, exponential correlation.

geneous correlation effects extend to analog MRC. We have derived the expected signal and interference power, demonstrating that SIR decreases when the user correlation matrices are identical, but increases when they are different. We derived asymptotic SINR expressions for both analog and digital MRC for benchmark scenarios of uncorrelated and fully correlated Rayleigh channels, demonstrating that the performance is critically dependent on the correlation scenario. We have shown that for uncorrelated fading the SINR converges to a constant. For fully correlated channels SINR converges to zero for equal correlation matrices, whereas for unequal correlation matrices SINR converges to zero or a constant, depending on the correlation model. 


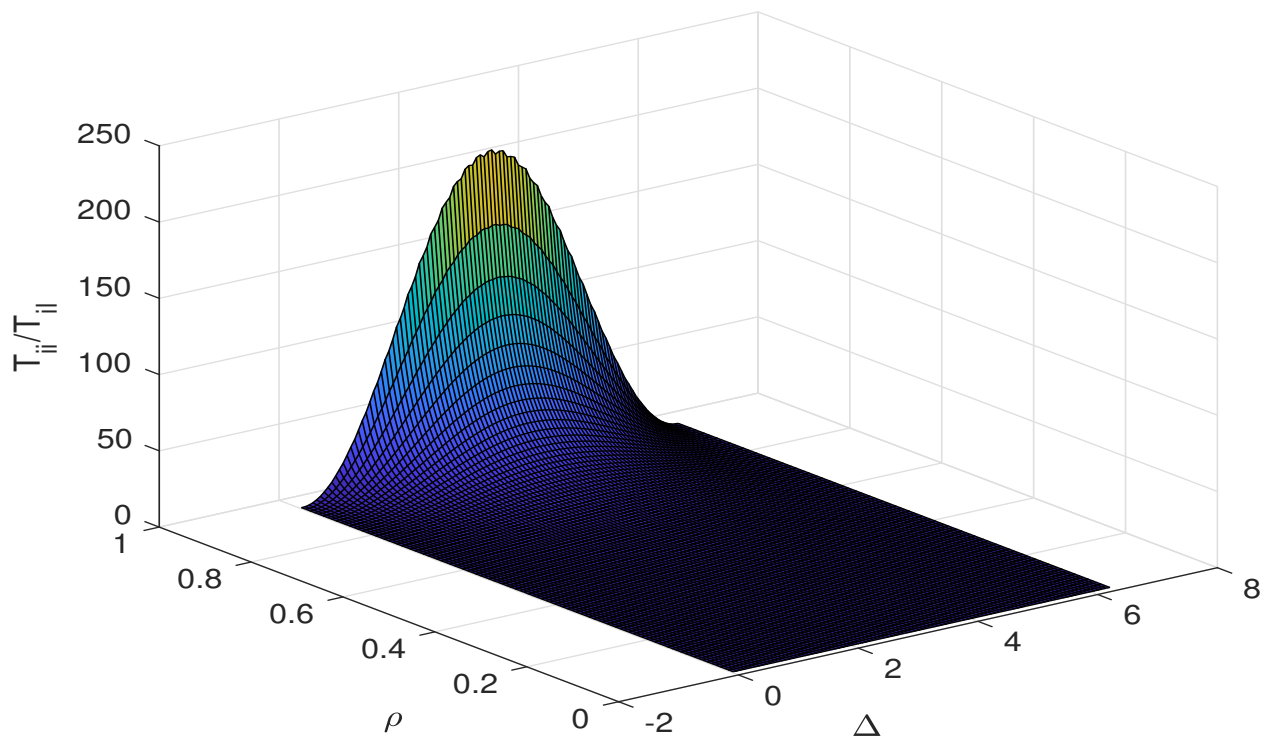

Figure 5.5: $T_{i i} / T_{i l}$ vs $\rho$ and $\Delta ; N=32$, exponential correlation.

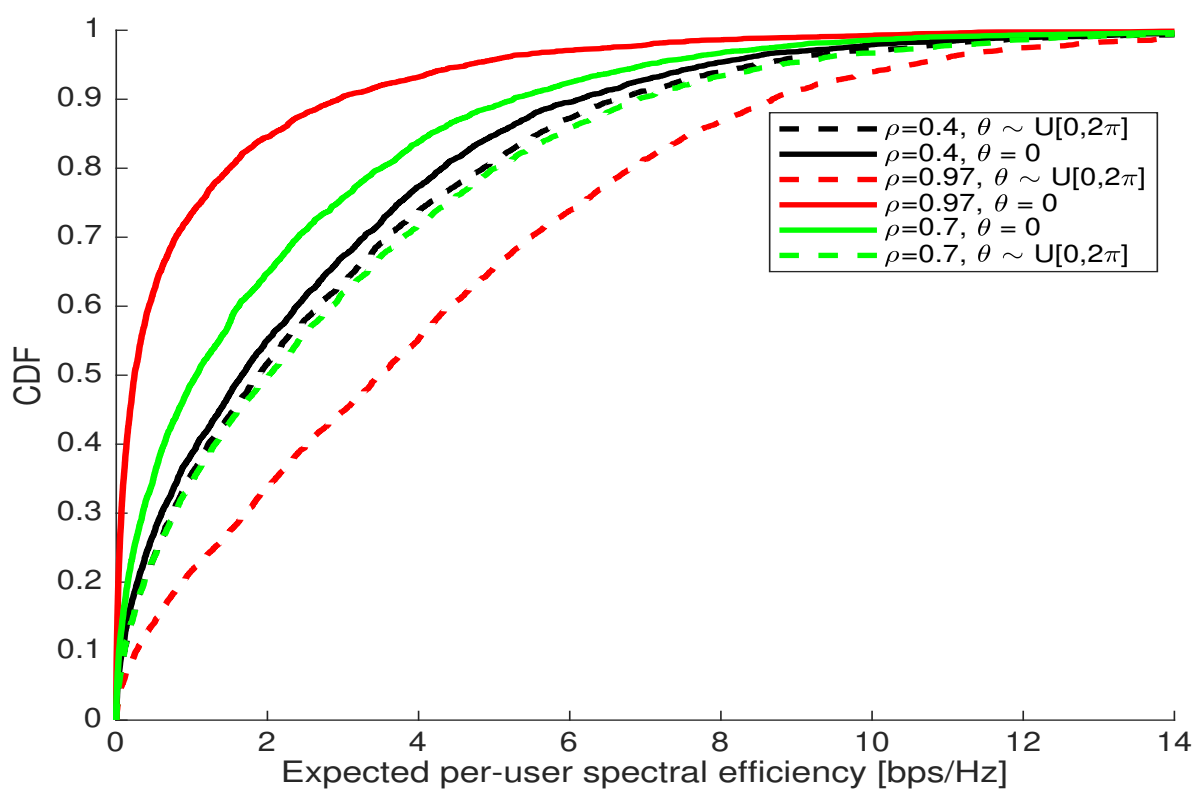

Figure 5.6: Spectral efficiency CDFs for fixed and uniformly distributed phase parameters and $N=32$. 
In this chapter, we present analytical expressions for the expected per user SINR in an uplink distributed multi-user multiple input multiple output system with two-stage beamforming. This leads to a tight approximation of expected per-user spectrum efficiency. System analysis is carried out for twoand four-cluster BS layouts, and four beamforming strategies. The local beamforming (first stage) considers either analog or digital MRC, followed by the global processing (second stage) of either digital MRC or digital ZF. We denote the resulting beamforming schemes as A-D, D-D, A-ZF and D-ZF. We also present the asymptotic behaviour of A-D and D-D strategies. Our analysis demonstrates a $\pi / 4$ performance ratio in a centralized configuration ${ }^{1}$. For the distributed layout, we show that the relative performance of A-D and D-D varies depending on the distribution of interference powers among the base stations. Here, when the dominant interferer powers are scattered among the BS clusters, D-D significantly outperforms A-D (with a gap greater than $\pi / 4)$. On the other hand, A-D outperforms D-D when the dominant interferer powers from all users happen to coincide at one BS cluster. Our analysis also shows that the MRC / ZF performance cross-over is delayed for two-stage beamforming (relative to single-stage), due to stage one reducing interference

\footnotetext{
${ }^{1}$ The performance ratio of AD to $\mathrm{DD}$ is $\pi / 4$ for centralized systems. The ratio is less than one as digital processing is more powerful.
} 
levels by a factor which is proportional to the number of antennas per cluster. Furthermore, we show that the performance gap between A-D and D-D increases with the number of BS clusters.

\subsection{Introduction}

MU-MIMO systems with a single BS deploy a large number of antennas in one physical location. Distributed systems (dividing the antennas into multiple antenna clusters) provide the benefits of reduced spatial correlation [22] and greater coverage than conventional co-located systems [13]. In fully cooperative distributed systems, where all the received signals are sent from the antenna clusters to a central processor, attractive performance gains are achieved compared to the equivalent non-cooperative network [22,33]. In [13], full cooperation is evaluated in the downlink with dirty paper coding and ZF. However, taking communication overheads and complexity into consideration, simplified processing is required. Hence, MRC with user grouping was proposed in [22]. To relieve the need for centralized knowledge of all the signals, two-stage processing is proposed in [33]. Here, only the output signals after local (first stage) processing are sent to the (second stage) central processing node. Using linear minimum mean squared error (MMSE) detection, [33] shows that the gap between the fully cooperative system and two-stage processing system is small.

In this paper, we adopt the promising two-stage procesing scheme and further reduce the complexity and hardware requirements of local and central processing by considering analog and digital MRC. These techniques require no central control [22] and analog MRC only requires phase-shifters. The performance analysis of analog and digital MRC for centralized and distributed MU-MIMO systems is given in [133]. We propose four types of two-stage 
processing, where either analog or digital MRC in stage one, is followed by digital MRC or digital ZF in stage two. We denote the resulting beamforming schemes as A-D, D-D, A-ZF and D-ZF.

To the best of our knowledge, there exist no closed-form SINR results for A-D, D-D in the uplink in a distributed MU-MIMO systems, nor any performance comparison with A-ZF and D-ZF. Hence, to fill this gap, we present a thorough analysis of the four two-stage BF processing techniques providing the following contributions:

- Novel analytical expressions for the expected SE for uplink A-D and D-D are derived.

- We analyse the system performance of A-D and D-D for two- and fourcluster BS layouts. We show that D-D benefits from increased cluster decentralization.

- Simulation results for A-ZF and D-ZF allow a performance comparison of A-D, D-D, A-ZF, and D-ZF. We show that MRC is a reasonable alternative to $\mathrm{ZF}$ in the second stage even at moderate SNR levels.

- We show that A-D has an asymptotic performance loss of approximately $21.5 \%$ compared to D-D in centralized systems. For distributed systems, the performance comparison depends on the pattern of user link gains.

\subsection{System Model}

Consider a MU-MIMO system with $K$ single antenna users randomly located in a single cell served by $N_{\mathrm{t}}$ BS antennas divided equally among $M$ antenna clusters. We consider two different BS layouts: four- and two-cluster. The antennas in the four-cluster distributed system are divided equally amongst four cooperative antenna sites with each site (half way from the centre to the 
cell-edge and $90^{\circ}$ from each other) equipped with $N=\frac{N_{t}}{4}$ antennas. Similarly, the two-cluster system has two sites, each (half way from the centre to the cell-edge and $180^{\circ}$ from each other) with $N=\frac{N_{t}}{2}$ antennas.

We consider an uplink system based on MMSE channel estimation with i.i.d Rayleigh fading. Based on the received pilot signal at one cluster, MMSE estimation is applied to obtain the channel estimates $\hat{\mathbf{h}}_{i}$ (the $N \times 1$ estimated channel vector for user $i$ ). The properties of the estimates are given in [134] and are summarized here. The true channel vector is denoted by $\mathbf{h}_{i}$ and has the distribution $\mathcal{C N}\left(\mathbf{0}, \Sigma_{i}\right)$, where $\Sigma_{i}=\beta_{i} \mathbf{I}$. The estimation error $\mathbf{e}_{i}=$ $\mathbf{h}_{i}-\hat{\mathbf{h}}_{i}$ has the covariance matrix $\mathbf{C}_{i}$. For i.i.d Rayleigh channels, $\mathbf{C}_{i}=\beta_{i} \mathbf{I}-$ $\gamma_{\mathrm{p}} \beta_{i}^{2}\left[\left(\gamma_{\mathrm{p}} \beta_{i}+1\right) \mathbf{I}\right]^{-1}=\beta_{i}^{2} /\left(1+\gamma_{\mathrm{p}} \beta_{i}\right) \mathbf{I}$, where $\gamma_{\mathrm{p}}=P \tau_{\mathrm{p}} / \sigma^{2}, \tau_{\mathrm{p}}$ is the number of pilots, $P$ is the transmit power, and $\sigma^{2}$ is the noise power. The resulting estimated channel has the distribution $\mathcal{C N}\left(\mathbf{0}, \mathbf{R}_{i}\right)$, where $\mathbf{R}_{i}=\tilde{\beta}_{i} \mathbf{I}$, $\tilde{\beta}_{i}=\gamma_{\mathrm{p}} \beta_{i}^{2} /\left(1+\gamma_{\mathrm{p}} \beta_{i}\right)$. We consider $\beta_{i}=A \zeta_{i}\left(d_{0} / d_{i}\right)^{\gamma}$, where $A$ is a unit-less constant representing the geometric attenuation at the reference distance $d_{0}, d_{i}$ is the distance between the $i^{\text {th }}$ user and the cluster, $\gamma$ is the path loss exponent and $\zeta_{i}$ is a log-normal random variable, $10 \log _{10} \zeta_{i} \sim \mathcal{N}\left(0, \sigma_{\text {sh }}^{2}\right)$, to model the effect of shadowing between the the $i^{\text {th }}$ user and the cluster. Equal transmit power for each user and narrow-band transmission are assumed throughout this work.

\subsection{Approximations of Achievable Uplink Spectral Efficiency}

In this section, we derive expressions for the expected per-user signal power and interference power for the A-D and D-D beamforming schemes. 


\subsubsection{Two-stage SINR for A-D and D-D}

The received signal at BS $k$ is given by

$$
\mathbf{r}_{k}=\sum_{j=1}^{K} \mathbf{h}_{j k} s_{j}+\mathbf{n}_{k}
$$

where $\mathbf{s}=\left\{s_{1}, s_{2}, \ldots, s_{K}\right\}^{\mathrm{T}}$ is the $K \times 1$ data symbol vector from the $K$ users and $\mathbb{E}\left[\mathbf{s s}^{\mathrm{H}}\right]=\mathbf{I} ; \mathbf{n}_{k}$ models the white Gaussian noise, $\mathbf{n}_{k} \sim \mathcal{C N}\left(\mathbf{0}, \sigma^{2} \mathbf{I}\right)$. At BS $k$ we have the channel estimates $\hat{\mathbf{h}}_{1 k}, \hat{\mathbf{h}}_{2 k}, \ldots, \hat{\mathbf{h}}_{K k}$. To detect user $i$ at BS $k$, the first stage uses the linear combiner $\mathbf{w}_{i k}$, which is designed based on the estimated channels, and here, can be either analog or digital MRC. Hence, the output signal at BS $k$ is

$$
\begin{aligned}
\tilde{r}_{k} & =\mathbf{w}_{i k}^{\mathrm{H}} \mathbf{r}_{k}=\mathbf{w}_{i k}^{\mathrm{H}}\left[\mathbf{h}_{i k} s_{i}+\sum_{j \neq i} \mathbf{h}_{j k} s_{j}+\mathbf{n}_{k}\right], \\
& =\mathbf{w}_{i k}^{\mathrm{H}} \hat{\mathbf{h}}_{i k} s_{i}+\mathbf{w}_{i k}^{\mathrm{H}} \mathbf{e}_{i k} s_{i}+\sum_{j \neq i} \mathbf{w}_{i k}^{\mathrm{H}} \mathbf{h}_{j k} s_{j}+\mathbf{w}_{i k}^{\mathrm{H}} \mathbf{n}_{k}, \\
& =\alpha_{i k} s_{i}+\delta_{i k} s_{i}+\sum_{j \neq i} \alpha_{i j k} s_{j}+v_{i k},
\end{aligned}
$$

where $\alpha_{i k}=\mathbf{w}_{i k}^{\mathrm{H}} \hat{\mathbf{h}}_{i k}, \delta_{i k}=\mathbf{w}_{i k}^{\mathrm{H}} \mathbf{e}_{i k}, \alpha_{i j k}=\mathbf{w}_{i k}^{\mathrm{H}} \mathbf{h}_{i j}$, and $v_{i k}=\mathbf{w}_{i k}^{\mathrm{H}} \mathbf{n}_{k}$. Stacking the outputs, $\tilde{r}_{k}, k=1,2, \ldots, M$, gives

$$
\tilde{\mathbf{r}}_{i}=\mathbf{a}_{i} s_{i}+\boldsymbol{\delta}_{i} s_{i}+\sum_{j \neq i} \mathbf{a}_{i j} s_{j}+\mathbf{v}_{i}
$$

where $\mathbf{a}_{i}=\left[\alpha_{i 1}, \alpha_{i 2}, \ldots, \alpha_{i M}\right]^{\mathrm{T}}, \boldsymbol{\delta}_{i}=\left[\delta_{i 1}, \delta_{i 2}, \ldots, \delta_{i M}\right]^{\mathrm{T}}, \mathbf{a}_{i j}=\left[\alpha_{i j 1}, \alpha_{i j 2}, \ldots, \alpha_{i j M}\right]^{\mathrm{T}}$, and $\mathbf{v}_{i}=\left[v_{i 1}, v_{i 2}, \ldots, v_{i M}\right]^{\mathrm{T}}$ represent the signal, channel estimation error, interference and noise terms, respectively. At the second stage, we perform digital 
processing with the combiner $\mathbf{u}_{i}$ to detect the desired signal $s_{i}$, giving

$$
\tilde{s}_{i}=\mathbf{u}_{i}^{\mathrm{H}} \tilde{\mathbf{r}}_{i}=\mathbf{u}_{i}^{\mathrm{H}} \mathbf{a}_{i} s_{i}+\mathbf{u}_{i}^{\mathrm{H}} \boldsymbol{\delta}_{i} s_{i}+\mathbf{u}_{i}^{\mathrm{H}} \sum_{j \neq i} \mathbf{a}_{i j} s_{j}+\mathbf{u}_{i}^{\mathrm{H}} \mathbf{v}_{i}
$$

From (6.4) we obtain the SINR for user $i$ as

$$
\operatorname{SINR}_{i}=\frac{\left|\mathbf{u}_{i}^{\mathrm{H}} \mathbf{a}_{i}\right|^{2} E_{\mathrm{s}}}{\mathbb{E}\left[\left|\mathbf{u}_{i}^{\mathrm{H}} \boldsymbol{\delta}_{i}\right|^{2}\right] E_{\mathrm{s}}+\sum_{j \neq i}\left|\mathbf{u}_{i}^{\mathrm{H}} \mathbf{a}_{i j}\right|^{2} E_{\mathrm{s}}+\mathbb{E}\left[\mathbf{u}_{i}^{\mathrm{H}} \mathbf{v}_{i} \mathbf{v}_{i}^{\mathrm{H}} \mathbf{u}_{i}\right]}
$$

After averaging over the signal, noise, and estimation error using standard results, (6.5) can be rewritten as

$$
\begin{aligned}
\operatorname{SINR}_{i} & =\frac{\left|\mathbf{u}_{i}^{\mathrm{H}} \mathbf{a}_{i}\right|^{2} E_{\mathrm{s}}}{\sum_{s=1}^{M}\left|u_{i s}\right|^{2} \mathbf{w}_{i s}^{\mathrm{H}}\left(E_{\mathrm{s}} \mathbf{C}_{i s}+\sigma^{2} \mathbf{I}\right) \mathbf{w}_{i s}+\sum_{j \neq i}\left|\mathbf{u}_{i}^{\mathrm{H}} \mathbf{a}_{i j}\right|^{2} E_{\mathrm{s}}} \\
& \triangleq S /\left(T_{1}+T_{2}\right) .
\end{aligned}
$$

In the following, we compute exact expressions for the mean signal, interference, estimation error, and noise powers.

\section{Expected Desired Signal Power}

$$
\begin{aligned}
\mathbb{E}[S] & =E_{\mathrm{s}} \mathbb{E}\left[\left|\mathbf{u}_{i}^{\mathrm{H}} \mathbf{a}_{i}\right|^{2}\right]=E_{\mathrm{s}} \mathbb{E}\left[\left|\mathbf{a}_{i}^{\mathrm{H}} \mathbf{a}_{i}\right|^{2}\right], \\
& =E_{\mathrm{s}} \sum_{r=1}^{M} \sum_{r=1}^{M} \mathbb{E}\left[\left|\mathbf{w}_{i r}^{\mathrm{H}} \hat{\mathbf{h}}_{i r}\right|^{2}\left|\mathbf{w}_{i s}^{\mathrm{H}} \hat{\mathbf{h}}_{i s}\right|^{2}\right], \\
& =E_{\mathrm{s}}\left\{\sum_{r=1}^{M} \mathbb{E}\left[\left|\mathbf{w}_{i r}^{\mathrm{H}} \hat{\mathbf{h}}_{i r}\right|^{4}\right]+\sum_{r \neq s} \mathbb{E}\left[\left|\mathbf{w}_{i r}^{\mathrm{H}} \hat{\mathbf{h}}_{i r}\right|^{2}\left|\mathbf{w}_{i s}^{\mathrm{H}} \hat{\mathbf{h}}_{i s}\right|^{2}\right]\right\} .
\end{aligned}
$$




\section{Expected Interference and Estimation Error Power}

Defining $E_{\mathrm{s}} \mathbf{C}_{i s}+\sigma^{2} \mathbf{I}=\varepsilon_{i s} \mathbf{I}$, we have

$$
\begin{aligned}
\mathbb{E}\left[T_{1}\right] & =\sum_{s=1}^{M} \mathbb{E}\left[\left|u_{i s}\right|^{2} \mathbf{w}_{i s}^{\mathrm{H}}\left(E_{\mathrm{s}} \mathbf{C}_{i s}+\sigma^{2} \mathbf{I}\right) \mathbf{w}_{i s}\right], \\
& =\sum_{s=1}^{M} \varepsilon_{i s} \mathbb{E}\left[\mathbf{w}_{i s}^{\mathrm{H}} \hat{\mathbf{h}}_{i s} \hat{\mathbf{h}}_{i s}^{\mathrm{H}} \mathbf{w}_{i s} \mathbf{w}_{i s}^{\mathrm{H}} \mathbf{w}_{i s}\right] .
\end{aligned}
$$

\section{Expected Noise Power}

$$
\begin{aligned}
\mathbb{E}\left[T_{2}\right] & =\sum_{j \neq i} E_{\mathrm{s}} \mathbb{E}\left[\left|\mathbf{u}_{i}^{\mathrm{H}} \mathbf{a}_{i j}\right|^{2}\right]=\sum_{j \neq i} E_{\mathrm{s}} \mathbb{E}\left[\left|\mathbf{a}_{i}^{\mathrm{H}} \mathbf{a}_{i j}\right|^{2}\right], \\
& =\sum_{j \neq i} E_{\mathrm{s}} \sum_{r=1}^{M} \beta_{j r} \mathbb{E}\left[\hat{\mathbf{h}}_{i r}^{\mathrm{H}} \mathbf{w}_{i r} \mathbf{w}_{i r}^{\mathrm{H}} \mathbf{w}_{i r} \mathbf{w}_{i r}^{\mathrm{H}} \hat{\mathbf{h}}_{i r}\right] .
\end{aligned}
$$

By defining, $M_{1}(i, r)=\mathbb{E}\left[\left|\mathbf{w}_{i r}^{\mathrm{H}} \hat{\mathbf{h}}_{i r}\right|^{2}\right], M_{2}(i, r)=\mathbb{E}\left[\left|\mathbf{w}_{i r}^{\mathrm{H}} \hat{\mathbf{h}}_{i r}\right|^{4}\right]$, and $M_{3}(i, r)=$ $\mathbb{E}\left[\hat{\mathbf{h}}_{i r}^{\mathrm{H}} \mathbf{w}_{i r} \mathbf{w}_{i r}^{\mathrm{H}} \mathbf{w}_{i r} \mathbf{w}_{i r}^{\mathrm{H}} \hat{\mathbf{h}}_{i r}\right]$, we can rewrite $\mathbb{E}[S], \mathbb{E}\left[T_{1}\right]$, and $\mathbb{E}\left[T_{2}\right]$ as

$$
\begin{aligned}
& \mathbb{E}(S)=E_{\mathrm{s}} \sum_{r=1}^{M} M_{2}(i, r)+E_{\mathrm{s}} \sum_{r \neq s} M_{1}(i, r) M_{1}(i, s) . \\
& \mathbb{E}\left(T_{1}\right)=\sum_{s=1}^{M} M_{3}(i, s) \varepsilon_{i s} . \\
& \mathbb{E}\left(T_{2}\right)=\sum_{j \neq i} E_{\mathrm{s}} \sum_{r=1}^{M} \beta_{j r} M_{3}(i, r) .
\end{aligned}
$$

Next, we require the values of $M_{1}(\cdot), M_{2}(\cdot)$, and $M_{3}(\cdot)$ for A-D and D-D processing. 


\section{A-D SINR}

For $\mathrm{A}-\mathrm{D}, \mathbf{w}_{i r}=\angle \hat{\mathbf{h}}_{i r}$, where $\angle \mathbf{p}=\left(p_{1} /\left|p_{1}\right|, p_{2} /\left|p_{2}\right|, \ldots, p_{n} /\left|p_{n}\right|\right)^{\mathrm{T}}$ for vector $\mathbf{p}=\left(p_{1}, p_{2}, \ldots, p_{n}\right)^{\mathrm{T}}$ records only the phase information in $\mathbf{p}$. Thus, $\mathbf{w}_{i r}^{\mathrm{H}} \hat{\mathbf{h}}_{i r}=$ $\tilde{\beta}_{i r}^{1 / 2} \sum_{q=1}^{N}\left|Z_{q}\right|$, where $Z_{q} \sim \mathcal{C N}(0,1)$ and $Z_{1}, Z_{2}, \ldots, Z_{N}$ are i.i.d. Using this formulation and $\mathbf{w}_{i r}^{\mathrm{H}} \mathbf{w}_{i r}=N$, gives

$$
\begin{aligned}
& M_{1}(i, r)=\tilde{\beta}_{i r}\{N+N(N+1) \pi / 4\} . \\
& M_{2}(i, r)=\tilde{\beta}_{i r}^{2}\{2 N+\{3 \pi / 2\} N(N-1)\} \\
& +\tilde{\beta}_{i r}^{2}\{3 N(N-1)+\{3 \pi / 2\} N(N-1)(N-2)\} \\
& +\tilde{\beta}_{i r}^{2}\left\{\left(\pi^{2} / 16\right) N(N-1)(N-2)(N-3)\right\} . \\
& M_{3}(i, r)=N \tilde{\beta}_{i r}\{N+N(N+1) \pi / 4\} .
\end{aligned}
$$

Defining $c_{1}(N)=N(3 N-1)+(3 \pi / 2) N(N-1)^{2}+\left(\pi^{2} / 16\right) N(N-1)(N-2)(N-$ $3), c_{2}(N)=N+N(N-1) \pi / 4$, and $c_{3}(N)=N[N+N(N-1) \pi / 4]$, the final expression is

$$
\frac{\mathbb{E}\left[\mathrm{SINR}_{i}^{\mathrm{AD}}\right] \approx}{E_{\mathrm{s}}\left\{c_{1}(N) \sum_{r=1}^{M} \tilde{\beta}_{i r}^{2}+c_{2}(N)\left(\left(\sum_{r=1}^{M} \tilde{\beta}_{i r}\right)^{2}-\sum_{r=1}^{M} \tilde{\beta}_{i r}^{2}\right)\right\}},
$$

using the approximation $\mathbb{E}[X / Y] \approx \mathbb{E}[X] / \mathbb{E}[Y]$ motivated by [105] and shown to be accurate for moderate to large numbers of antennas $[105,133]$. Thus, we have the SE for user $i$ as

$$
\mathbb{E}\left[\mathrm{SE}_{i}\right] \approx \log _{2}\left(1+\mathbb{E}\left[\mathrm{SINR}_{i}^{\mathrm{AD}}\right]\right)
$$




\section{D-D SINR}

For D-D, $\mathbf{w}_{i r}=\hat{\mathbf{h}}_{i r}$ and thus $\mathbf{w}_{i r}^{\mathrm{H}} \hat{\mathbf{h}}_{i r}=\frac{1}{2} \tilde{\beta}_{i r} \chi_{i r}$, where $\chi_{i r} \sim \chi_{2 N}^{2}$ is a Chisquared random variable with $2 N$ degrees of freedom. The moments of $\chi_{i r}$ are known, so we can write $M_{k}(i, r), k=1,2,3$ for D-D as follows:

$$
\begin{aligned}
& M_{1}(i, r)=\tilde{\beta}_{i r}^{2} \mathbb{E}\left[\chi_{i r}^{2}\right]=\tilde{\beta}_{i r}^{2} N(N+1) . \\
& M_{2}(i, r)=\tilde{\beta}_{i r}^{4} \mathbb{E}\left[\chi_{i r}^{4}\right]=\tilde{\beta}_{i r}^{4} N(N+1)(N+2)(N+3) . \\
& M_{3}(i, r)=\tilde{\beta}_{i r}^{3} \mathbb{E}\left[\chi_{i r}^{3}\right]=\tilde{\beta}_{i r}^{3} N(N+1)(N+2) .
\end{aligned}
$$

The final expression can then be written as

$$
\begin{aligned}
& \mathbb{E}\left[\mathrm{SINR}_{i}^{\mathrm{DD}}\right] \\
& \approx \frac{E_{\mathrm{s}}\left\{b_{1}(N) \sum_{r=1}^{M} \tilde{\beta}_{i r}^{4}+b_{2}(N)\left(\left(\sum_{r=1}^{M} \tilde{\beta}_{i r}^{2}\right)^{2}-\sum_{r=1}^{M} \tilde{\beta}_{i r}^{4}\right)\right\}}{b_{3}(N) \sum_{s=1}^{M} \varepsilon_{i s} \tilde{\beta}_{i s}^{3}+E_{\mathrm{s}} b_{3}(N) \sum_{j \neq i} \sum_{r=1}^{M} \beta_{j r} \tilde{\beta}_{i r}^{3}},
\end{aligned}
$$

where $b_{1}(N)=(N+2)(N+3), b_{2}(N)=N(N+1)$, and $b_{3}(N)=N+2$. Similarly, we have the SE for user $i$ as

$$
\mathbb{E}\left[\mathrm{SE}_{i}\right] \approx \log _{2}\left(1+\mathbb{E}\left[\mathrm{SINR}_{i}^{\mathrm{DD}}\right]\right)
$$

\subsubsection{Asymptotic Analysis of A-D and D-D}

In this section, we derive the performance gap between $A-D$ and D-D when $N$ grows large. Note that in Sec. 6.3.1, $c_{1}(N)$ and $c_{2}(N)$ are quartic in $N$ and $c_{3}(N)$ is cubic. Hence, (6.14) can be rewritten as

$$
\mathbb{E}\left[\mathrm{SINR}_{i}^{\mathrm{AD}}\right] \sim(\pi / 4) \eta_{\mathrm{AD}} N
$$


where $\eta_{\mathrm{AD}}=\frac{E_{\mathrm{s}}\left(\sum_{r=1}^{M} \tilde{\beta}_{i r}^{2}\right) N}{\sum_{s=1}^{M} \varepsilon_{i s} \tilde{\beta}_{i s}+E_{\mathrm{s}} \sum_{j \neq i} \sum_{r=1}^{M} \beta_{j r} \tilde{\beta}_{i r}}$. Similarly, for large $N$,

$$
\mathbb{E}\left[\mathrm{SINR}_{i}^{\mathrm{DD}}\right] \sim \eta_{\mathrm{DD}} N
$$

where $\eta_{\mathrm{DD}}=\frac{E_{\mathrm{s}}\left\{\sum_{r=1}^{M} \tilde{\beta}_{i r}^{4}+\left(\sum_{r=1}^{M} \tilde{\beta}_{i r}^{2}\right)^{2}-\sum_{r=1}^{M} \tilde{\beta}_{i r}^{4}\right\}}{\sum_{s=1}^{M} \varepsilon_{i s} \tilde{\beta}_{i s}^{3}+E_{\mathrm{s}} \sum_{j \neq i} \sum_{r=1}^{M} \beta_{j r} \tilde{\beta}_{i r}^{3}}$. For a co-located system

$\eta_{\mathrm{AD}}$ and $\eta_{\mathrm{DD}}$ simplify to give

$$
\lim _{N \rightarrow \infty} \frac{\mathbb{E}\left[\mathrm{SINR}_{i}^{\mathrm{AD}}\right]}{\mathbb{E}\left[\mathrm{SINR}_{i}^{\mathrm{DD}}\right]} \sim \frac{\pi}{4}
$$

For a distributed system, the ratio in (6.21) changes according to variation in the link gains. This is explained in Sec. 6.4.

\subsubsection{A-ZF and D-ZF SINR}

Due to the intractable nature of ZF at the second stage, we only derive an SINR expression for ZF and use simulations to average over the fast fading. We first rewrite (6.3) as

$$
\tilde{\mathbf{r}}_{i}=\mathbf{a}_{i} s_{i}+\sum_{j \neq i} \mathbf{f}_{i j} s_{j}+\boldsymbol{\delta}_{i} s_{i}+\sum_{j \neq i} \mathbf{g}_{i j} s_{j}+\mathbf{v}_{i}
$$

using $\mathbf{a}_{i j}=\mathbf{f}_{i j}+\mathbf{g}_{i j}, \mathbf{f}_{i j}=\left[\mathbf{w}_{i 1}^{\mathrm{H}} \hat{\mathbf{h}}_{j 1}, \ldots, \mathbf{w}_{i M}^{\mathrm{H}} \hat{\mathbf{h}}_{j M}\right]^{\mathrm{T}}$, and $\mathbf{g}_{i j}=\left[\mathbf{w}_{i 1}^{\mathrm{H}} \mathbf{e}_{j 1}, \ldots, \mathbf{w}_{i M}^{\mathrm{H}} \mathbf{e}_{j M}\right]^{\mathrm{T}}$. The first two terms in (6.22) represent the known coefficients of $\mathbf{s}=\left[s_{1}, s_{2}, \ldots, s_{K}\right]^{\mathrm{T}}$ and are written as $\mathbf{A}_{i} \mathbf{s}$. The remaining terms in (6.22) are due to noise and estimation error and are denoted by the vector, $\boldsymbol{\eta}_{i}$. Hence, $\tilde{\mathbf{r}}_{i}=\mathbf{A}_{i} \mathbf{s}+\boldsymbol{\eta}_{i}$ and standard ZF gives the combiner $\mathbf{u}_{i}$ as the $i^{\text {th }}$ column of $\mathbf{A}_{i}\left(\mathbf{A}_{i}^{\mathrm{H}} \mathbf{A}_{i}\right)^{-1}$ with the SINR 


$$
\operatorname{SINR}_{i}^{\mathrm{ZF}}=\frac{E_{s}}{\mathbb{E}\left[\left|\mathbf{u}_{i}^{\mathrm{H}} \boldsymbol{\eta}_{i}\right|^{2}\right]}=\frac{E_{s}}{\mathbf{u}_{i}^{\mathrm{H}} \mathbb{E}\left[\boldsymbol{\eta}_{i} \boldsymbol{\eta}_{i}^{\mathrm{H}}\right] \mathbf{u}_{i}},
$$

where the expectation in (6.23) is over signal, noise, and estimation error. Using standard properties of the noise term, $\mathbf{v}_{i}$, and the estimation errors, $\mathbf{e}_{j k}$, in $\boldsymbol{\eta}_{i}$ we obtain $\mathbb{E}\left[\boldsymbol{\eta}_{i} \boldsymbol{\eta}_{i}^{\mathrm{H}}\right]=\mathbf{Q}_{i}$, where $\mathbf{Q}_{i}=\operatorname{diag}\left(q_{i 1}, q_{i 2}, \ldots, q_{i M}\right)$ and $q_{i r}=\mathbf{w}_{i r}^{\mathrm{H}}\left(E_{\mathrm{s}} \sum_{j=1}^{K} \mathbf{C}_{j r}+\sigma^{2} \mathbf{I}\right) \mathbf{w}_{i r}$. Hence, we have

$$
\operatorname{SINR}_{i}^{\mathrm{ZF}}=\frac{E_{s}}{\mathbf{u}_{i}^{\mathrm{H}} \mathbf{Q}_{i} \mathbf{u}_{i}}
$$

Substituting $\mathbf{w}_{i r}=\angle \hat{\mathbf{h}}_{i r}$ in $\mathbf{u}_{i}$ and $\mathbf{Q}_{i}$ in (6.24) gives $\operatorname{SINR}_{i}^{\mathrm{A}-Z \mathrm{~F}}$ and using $\mathbf{w}_{i r}=$ $\hat{\mathbf{h}}_{i r}$ in $\mathbf{u}_{i}$ gives $\mathrm{SINR}_{i}^{\mathrm{D}-\mathrm{ZF}}$ and the resulting mean SE, $\mathbb{E}\left[\mathrm{SE}_{i}\right]=\mathbb{E}\left[\log _{2}\left(1+\mathrm{SINR}_{i}^{\mathrm{ZF}}\right)\right]$.

\subsection{Numerical Results}

In this section, the following parameters are used. Four users, each with a single antenna, are uniformly located in a circular cell with a radius of 100 meters and reference distance, $d_{0}=1$ meter. The pathloss exponent $\gamma=3.5$ and the standard deviation of shadowing $\zeta=6 \mathrm{~dB}$. The $\operatorname{SNR}\left(\mathbb{E}\left[\left|s_{i}\right|^{2}\right] / \sigma^{2}\right)$ is set to $15 \mathrm{~dB}$. The unit-less geometric attenuation $A$ is chosen to guarantee that the median of the desired user's SINR is $8 \mathrm{~dB}$ with the system of A-D processing, $N_{\mathrm{t}}=64$ and $M=4$.

In Fig. 6.1, we show a performance comparison based on the CDFs of $\mathbb{E}[\mathrm{SE}]$. The expectation is computed over the fast-fading and the CDF depicts the impact of the variations in large-scale fading. We see that the analysis in (6.15) and (6.18) agrees well with simulation results for six different system settings. With increasing numbers of clusters, the performance gap between A-D and D-D becomes bigger. This is because digital MRC will benefit 
from more distributed systems as it can cater for the large variations in signal strength across the clusters. This also explains why the system having A-D four clusters with 16 antennas per cluster has almost the same performance as the system having D-D 2 clusters with 32 antennas per cluster.

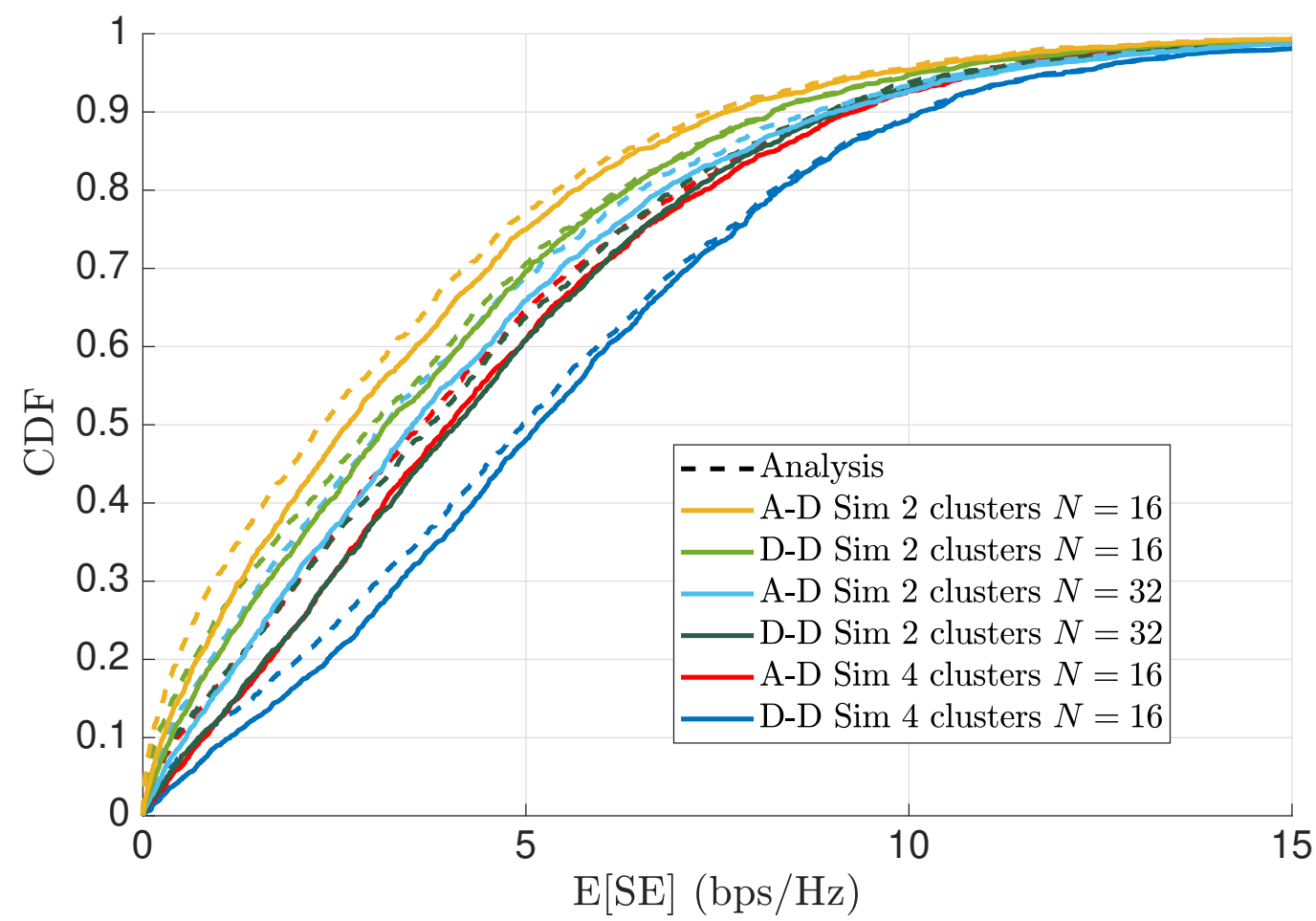

Figure 6.1: Performance comparison of expected per-user SE CDFs between A-D and D-D.

In Fig. 6.2, we demonstrate the performance cross-over between MRC and $\mathrm{ZF}$ based second stage processing, using $\mathbb{E}\left[\mathrm{SE}_{\text {cell }}\right]$, defined as the expected per user SE averaging over the cell. Four clusters with 16 antennas in each cluster and four users are considered. For traditional MU-MIMO systems with small numbers of antennas ZF is usually far superior to MRC. The exception is at very low SNR where the interference becomes small and it is more important to boost signal strength making MRC preferable. This superiority of MRC is not usually helpful as the low SNRs mean that system performance is too low. However, in our system stage one processing achieves consider- 
able interference reduction which helps MRC. For simplicity consider stage one with perfect CSI, analog MRC and normalized channels. The mean desired signal power is $\mathbb{E}\left[\left|\mathbf{h}_{i}^{\mathrm{H}} \mathbf{h}_{i}\right|^{2}\right]=N+N(N+1) \pi / 4$ (from (6.11)) and the mean interference power from user $j$ is $\mathbb{E}\left[\left|\mathbf{h}_{i}^{\mathrm{H}} \mathbf{h}_{j}\right|^{2}\right]=N$. Hence, stage one reduces interference levels relative to the signal by a factor proportional to $N$ (with the same trend for digital MRC). Due to this, MRC retains better or similar performance to ZF in stage two even at reasonable SNR levels up to $5 \mathrm{~dB}$ as shown in Fig. 6.2. Note that the $\mathrm{ZF}$ techniques remove interference completely with perfect CSI, so their performance has no ceiling. In contrast, the MRC only methods are interference limited and their performance has a finite limit as the SNR is increased.

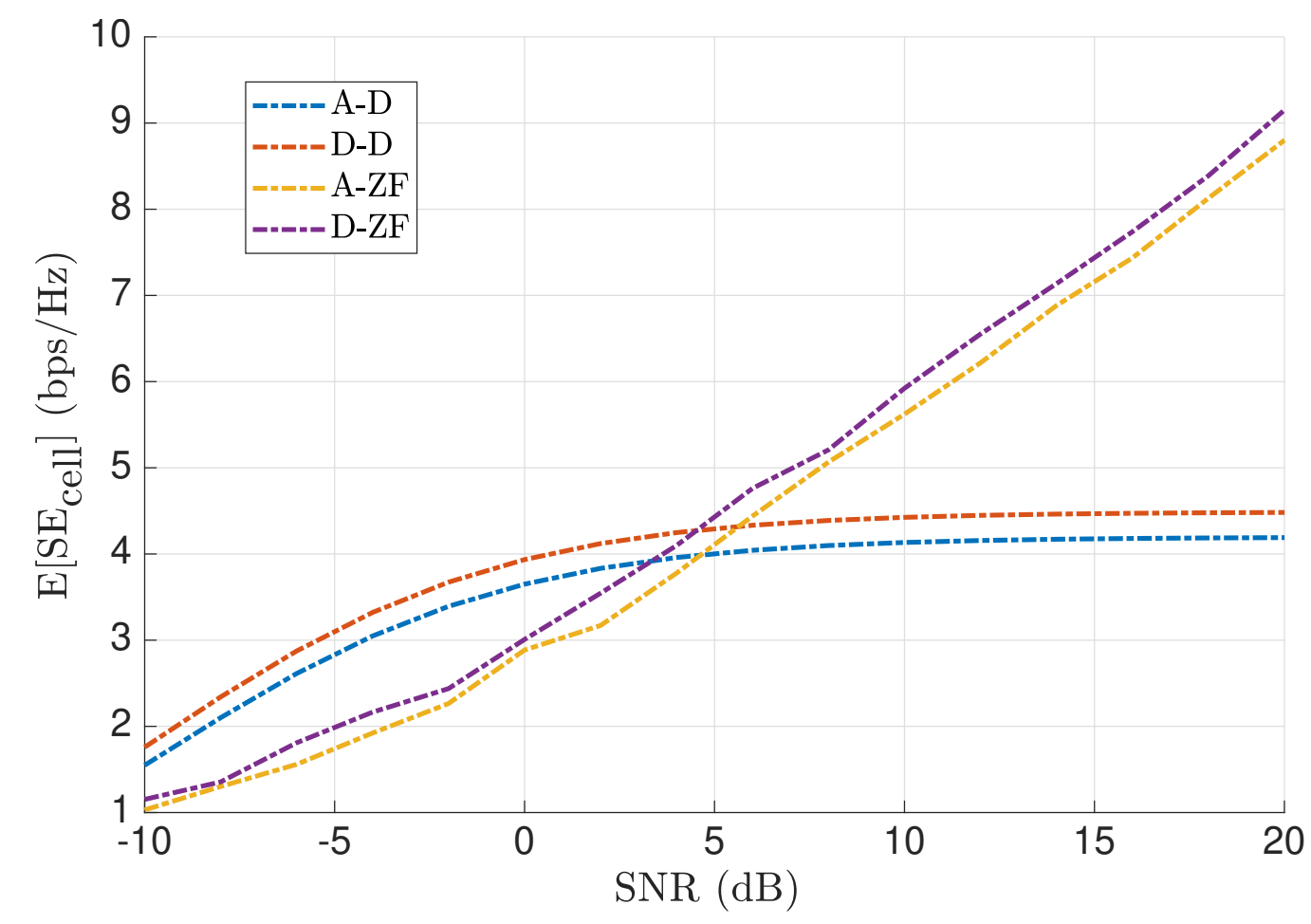

Figure 6.2: Expected per-user $\mathrm{SE}_{\text {cell }}$ vs $\mathrm{SNR}$ for four two-stage processing techniques. 
In Fig. 6.32, we illustrate the asymptotic analysis in (6.19)-(6.21). We use the same system parameters as in Fig. 6.2 except for the values of $\beta_{i j}$. $\mathbb{E}[\mathrm{SINR}]$ is defined in (6.14) and (6.17) for A-D and D-D systems, respectively. For the co-located (COL) system with equal link gain scenario, we have $\beta_{i j}=1, \forall i, j$ and the desired user is user 1 . From Fig. 6.3, we see a ratio of $\pi / 4$ between A-D and D-D. In distributed scenarios, the performance of A-D and D-D depends on the profile of the links gains. Close inspection of (6.19)-(6.20) shows that the performance of D-D relative to A-D is increased when the desired user's maximum link gain does not align with the interfering users' maximum link gain (Scenario 1). This is shown in Fig. 6.3 using the values $\boldsymbol{\beta}_{1}=(1.6,0.4,0.8,1.2), \boldsymbol{\beta}_{2}=(0.4,1.2,1.6,0.8), \boldsymbol{\beta}_{3}=(0.4,1.2,0.8,1.6)$, $\boldsymbol{\beta}_{4}=(0.8,1.6,1.2,0.4)$, where $\boldsymbol{\beta}_{i}=\left(\beta_{i 1}, \beta_{i 2}, \beta_{i 3}, \beta_{i 4}\right)$. Here D-D offers a substantial improvement over A-D. For the opposite scenario (Scenario 2) where the maximum link gains of users occur at the same cluster, we use $\boldsymbol{\beta}_{1}=$ $(1.6,1.2,0.8,0.4), \boldsymbol{\beta}_{2}=(1.6,1.2,0.4,0.8), \boldsymbol{\beta}_{3}=(1.6,0.8,0.4,1.2)$, $\boldsymbol{\beta}_{4}=(1.6,0.4,0.8,1.2)$. Note that both scenarios use a linearly decaying set of link gains with the same unit mean as the co-located case. For Scenario 2, (6.19)-(6.20) show that the performance of A-D is improved relative to D-D and Fig. 6.3 shows that A-D can outperform D-D. Note that the interference term in A-D is quadratic, with the interfering user's link gain scaled by the desired link gain. Hence, when the strong link gains align, interference is boosted. However, for D-D the interference term is quartic, with the interfering link gain scaled by the cube of the desired gain. Hence, the effects of aligned link gains are far more pronounced for D-D.

\footnotetext{
${ }^{2}$ The marker on the plot specifies that the AD to DD performance ratio is $\pi / 4$ for the colocated case. Numerically the ratio is very close to the exact value of $\pi / 4$.
} 


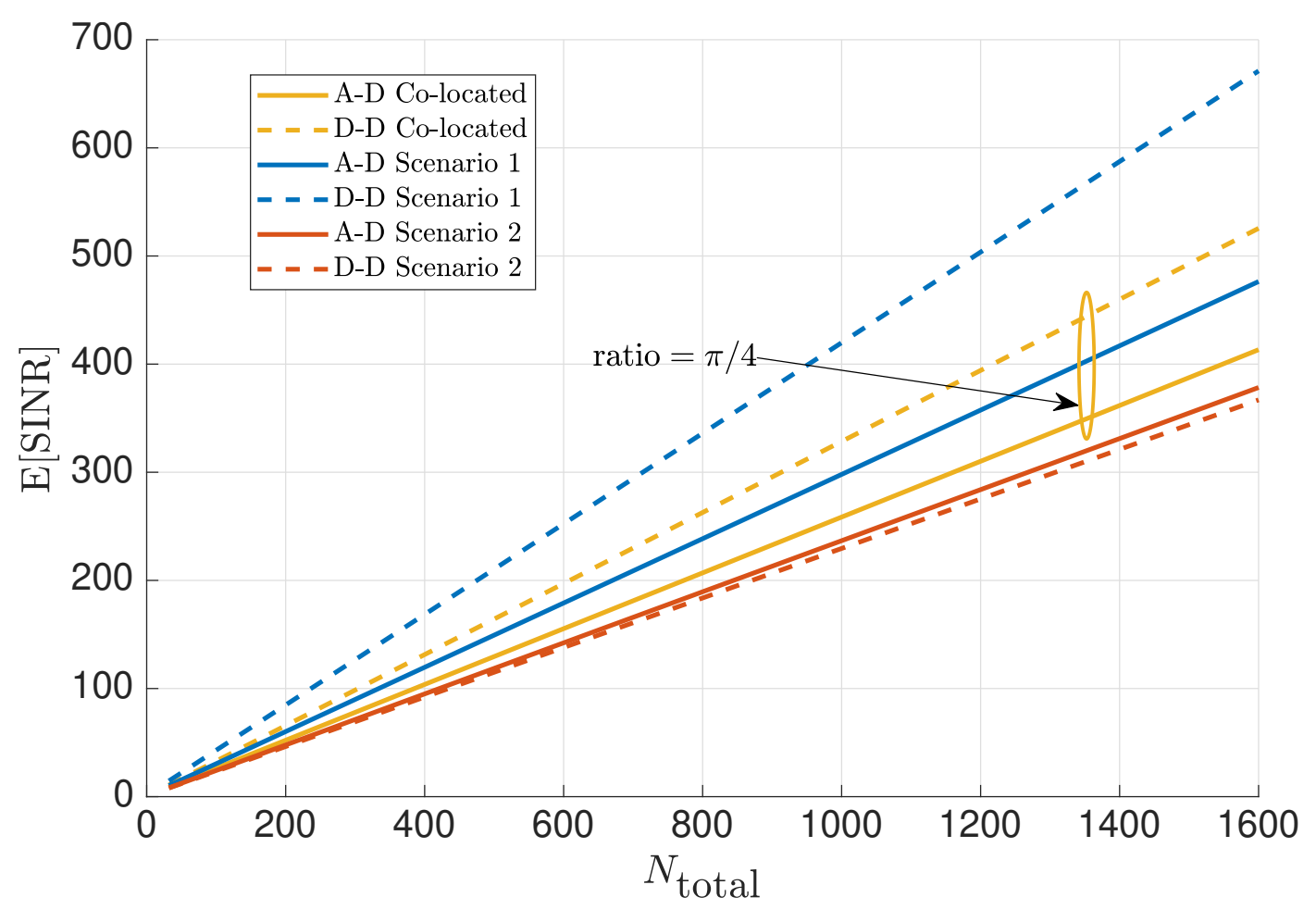

Figure 6.3: Expected per-user SINR vs $N_{\text {total }}$ for A-D and D-D in both colocated and distributed systems.

\subsection{Conclusion}

In this chapter, we have analysed four low-complexity two-stage BF techniques for a distributed system. We derive novel analytical expressions for the SE for uplink A-D and D-D. The derivation is robust to changes in system dimension. The system performance of A-D and D-D for two- and fourcluster BS layouts is analysed, showing that -D benefits from increased cluster decentralization. We show that A-D has an asymptotic performance loss of approximately $21.5 \%$ compared to D-D in centralized systems. For distributed systems, the performance comparison depends on the pattern of user link gains. We also show that MRC is a reasonable alternative to ZF in the second stage even at moderate SNR levels. 


\section{Massive MIMO for Ray-Based Channels}

Favourable propagation and channel hardening are desired properties in massive MIMO systems. To date, these properties have primarily been analyzed for classical statistical channel models, or ray-based models with very specific angular parameters and distributions. This chapter presents a thorough mathematical analysis of the asymptotic system behavior for ray-based channels with arbitrary ray distributions, and considers two types of antenna array structures at the cellular base station: a uniform linear array and a uniform planar array (UPA). In addition to FP and channel hardening, we analyze the LSP which measures the asymptotic ratio of the expected power in the desired channel to the expected total interference power when both the antenna and user numbers grow. LSP is said to hold when this ratio converges to a positive constant. The results demonstrate that while FP is guaranteed in ray-based channels, channel hardening may or may not occur depending on the nature of the model. Furthermore, we demonstrate that LSP will not normally hold as the expected interference power grows logarithmically for both ULAs and UPAs relative to the power in the desired channel as the system size increases. Nevertheless, we identify some fundamental and attractive properties of massive MIMO in this limiting regime. 


\subsection{Introduction}

Two key principles behind the success of massive MIMO are FP [3,38], and channel hardening [75], meaning that the normalized inter-user interference power converges to zero, and that the normalized power in the desired channel becomes constant. With FP, the use of large numbers of antennas offers an implicit interference reduction mechanism, and enables the use of low complexity signal processing algorithms [3,7,135].

The bulk of the theoretical work on FP and channel hardening has utilized classical statistical channel models. Here, the existence of FP has been demonstrated for channel models of increasing complexity, progressing from i.i.d. Rayleigh [38, 136], pure line-of-sight [38, 136], correlated Rayleigh [137, 138], and independent Ricean [139] to correlated Ricean channels [140,141]. In parallel, with the theory, channel measurements have demonstrated that a large fraction of the theoretical gains due to FP can be obtained $[8,142,143]$.

This work is now mature, but incomplete in the sense that accurate modeling of large dimensional channels requires a strong link to the propagation environment. This is usually obtained through ray-based models which have been extensively validated by measurements and, for this reason, have made their way to the 3GPP standardization process [35]. These models better capture the physics of electromagnetic propagation, and have a closer link to the array architecture and are widely used irrespective of the frequency band [35-37]. The physical nature of the ray-based models also has some advantages for system performance analysis in the sense that the analytical conclusions are based on physical features of the system rather than statistical modeling assumptions. For example, FP was considered in the recent work [140] for very general heterogeneous, correlated Ricean channels. This work gives wide ranging results on FP, but the inherent nature of these mod- 
els meant that the conclusions relied on various assumptions concerning the correlation structure, line-of-sight direction, etc. In contrast, we are able to prove FP for ray-based models with the most basic assumptions pertaining to physical phenomena, such as distributions of ray directions.

Variations of such models have a proliferation of names including directional, spatial and Saleh-Valenzulela (SV) type [48] channel models. We prefer the phrase ray-based, as the main requirement for our work is that the statistical distributions of individual rays can be identified and analyzed ${ }^{1}$. This is possible for a wide range of such channels. Important work has begun in this area demonstrating the existence of FP with specific ray-based models for a variety of antenna topologies, such as the ULA, UPA, and uniform circular array (UCA) [144], [145]. However, the majority of this work relies on two very special cases for the rays: an arbitrary ray must arrive with an azimuth angle, $\phi$, which satisfies $\phi \sim U[0,2 \pi][144-146]$ or $\sin \phi \sim U[-1,1][9,38]$. FP has also been demonstrated in [147] for the more complex case where azimuth angles have a uniform central angle and wrapped Gaussian sub rays and the elevation angles are Laplacian. It is, critical $^{2}$ to be able to predict the system performance with practical, and more general distributions, making our extension an important one in the context of our understanding of massive MIMO behavior. Hence, a general analysis of FP for ray-based models with arbitrary ray distributions is almost entirely lacking. Further, while FP is a desirable property for a communication system, it only implies that a finite number of users can be served by increasing the number of antenna elements. We refer to this as single-sided massive MIMO [144]. Ideally, as you grow the number of BS antennas you would also serve more users, leading to a sys-

\footnotetext{
${ }^{1}$ We prefer this terminology, since our primary interest is not in identifying distributions of rays across multiple clusters.

${ }^{2}$ This is important as unrealistic assumptions can lead to the opposite conclusions. For example, the assumption that $\sin \phi \sim U[-1,1]$ leads to the conclusion that the total interference in a massive MIMO system converges. In contrast, we show in Theorem 1 that interference diverges for realistic ray distributions.
} 
tem that becomes large both in users and antennas, i.e., double-sided massive MIMO, the concept of which is defined in [148]. Hence, we define LSP as the property that the fundamental ratio which measures the mean power in the desired channel relative to the total mean interference power converges to a positive constant as both the number of users $(K)$ and the number of antennas $(N)$ grow to infinity, with $N / K \rightarrow \alpha$ as $N \rightarrow \infty$. The analysis of LSP for i.i.d. Rayleigh fading can be found in [7], although it is not explicitly defined. In the first part of this chapter (and in [149]), channel hardening, FP, and LSP analysis for ray-based channel models and a basic ULA antenna structure are discussed. In the second part of the chapter, we extend this work to a UPA structure. This is an important, yet non trivial, extension as the addition of the elevation component in the ray based channel facilitates much greater accuracy in predicting massive MIMO behavior, something which is rare in the literature. Furthermore, the majority of the ongoing deployments of massive MIMO in the C-band utilize UPAs, in order to effectively leverage the full dimensional nature of the channel, and to maximize the beamforming gain of the system via reciprocity-based processing. The mathematical complexity of such an extension is also substantial, since our main aim is to obtain detailed insights into the desirable properties of massive MIMO systems with a model which is more closely in line with practice. We make the following contributions for both ULA and UPA topologies:

- We show that channel hardening may or may not occur depending on the nature of the model.

- We show that FP is guaranteed for all models where the ray angles are continuous random variables (as assumed by all models to date).

- For LSP, we derive remarkably simple expressions which relate the asymptotic interference behavior to system size, antenna spacing and the ray 
distribution. We demonstrate that LSP will not normally hold as the mean interference power grows logarithmically relative to the mean power of the desired user channel as the system size increases.

- Analytical results are verified via simulation and using special cases derived for specific angular distributions.

- Despite the lack of LSP, the implications for massive MIMO are excellent. Although the interference eventually dominates the desired channel, the growth is very slow and is further attenuated by practical factors such as the likely propagation environment and the typical array patterns employed. In addition, we prove that trivial scheduling schemes can retain LSP, and thus increase the robustness of massive MIMO performance.

\subsection{Channel Model and System Metrics}

We consider an uplink massive MIMO system with $N$ co-located antennas at one BS simultaneously serving $K$ single antenna users, where, for now, $N \gg K$. We assume a narrowband flat fading channel model such that the $N \times 1$ channel vector for user $i$ can be written as $\mathbf{h}_{i}$, and the composite $N \times K$ channel matrix is denoted by $\mathbf{H}=\left[\mathbf{h}_{1} \mathbf{h}_{2} \ldots \mathbf{h}_{K}\right]$. We assume that the propagation channel is known to both the users and the cellular BS. This is an assumption in this study since we are concerned with analyzing the fundamental properties of a massive MIMO system using ray-based channels. In practice, reciprocity-based beamforming may be used where uplink channel estimates will be used for payload data transmission. 


\subsubsection{Ray-based Channel Model}

In general, the propagation channel to user $i$ can be described as the superposition of many individual rays possibly arriving in clusters from a set of far-field scatterers. In simple terms, the channel is broken down into $P$ incident rays at the $\mathrm{BS}^{3}$. Hence, for a ULA we have:

$$
\mathbf{h}_{i}=\sum_{r=1}^{P} \gamma_{i r} \mathbf{a}\left(\phi_{i r}\right)
$$

as described in Chapter 2 (see Fig. (2.3)), $\phi_{i r}$ is the azimuth angle of the $r^{\text {th }}$ ray, $\gamma_{i r}$ is a complex scaling factor for the magnitude and phase of the ray, and $\mathbf{a}\left(\phi_{i r}\right)$ is the $N \times 1$ array steering vector. In azimuth, the antenna array broadside is at $\phi_{i r}=0$, and end-fire is $\phi_{i r}= \pm \frac{\pi}{2}$. Common models for the scaling factor include random phase models [35], where $\gamma_{i r}=\sqrt{\beta_{i r}} \exp \left(j \Phi_{i r}\right)$, $\beta_{i r}$ is the power of the $r^{\text {th }}$ ray and $\Phi_{i r}$ are i.i.d. $U[0,2 \pi]$ phase offsets. Hence, $\beta_{i}=\sum_{r=1}^{P} \beta_{i r}$ is the total link gain for user $i$. Also, complex Gaussian models have been proposed in [37], where $\gamma_{i r}=\sqrt{\beta_{i r}} u_{i r}$ and $u_{i r} \sim \mathcal{C N}(0,1)$. For both models, we note that $\mathbb{E}\left[\gamma_{i r}\right]=0, \mathbb{E}\left[\left|\gamma_{i r}\right|^{2}\right]=\beta_{i r}$ and $\mathbb{E}\left[\gamma_{i r}^{*} \gamma_{j s}\right]=0$ for all pairs $(i, r) \neq(j, s)$. For a ULA with normalized inter-element spacing $d$, measured in wavelengths, the steering vector is:

$$
\mathbf{a}\left(\phi_{i r}\right)=\left[1, e^{2 \pi j d \sin \phi_{i r}}, e^{2 \pi j 2 d \sin \phi_{i r}}, \ldots, e^{2 \pi j(N-1) d \sin \phi_{i r}}\right]^{\mathrm{T}}
$$

For a UPA, the total number of antennas, $N$, is divided into $N_{x}$ and $N_{y}$ antennas in the $x$ and $y$ axes with inter-element spacings $d_{x}$ and $d_{y}$, respectively.

\footnotetext{
${ }^{3}$ For ease of notation, we do not specifically itemize clusters, but the $P$ paths include any clustered rays. We note that since we are concerned with angular distributions of individual rays contributing to the channel impulse response, it is not necessary to categorize the channel model in terms of clusters. Note that later analysis usually simplifies to a single ray from two different users, so it does not matter how each ray is identified as a single ray using a single subscript is equivalent to a single ray using a double subscript.
} 
The steering vectors can be represented by the Kronecker product of $\left(N_{x} \times 1\right)$ $\mathbf{a}_{x}$ and $\left(N_{y} \times 1\right) \mathbf{a}_{y}$, which gives an $N_{x} N_{y} \times 1$ vector, $\mathbf{a}\left(\theta_{i r}, \phi_{i r}\right)=\mathbf{a}_{i r x} \otimes \mathbf{a}_{i r y}$. Note that

$$
\mathbf{a}_{i r x}=\left[1, e^{2 \pi j d_{x} \sin \theta_{i r} \cos \phi_{i r}}, \ldots, e^{2 \pi j\left(N_{x}-1\right) d_{x} \sin \theta_{i r} \cos \phi_{i r}}\right]^{\mathrm{T}},
$$

and

$$
\mathbf{a}_{i r y}=\left[1, e^{2 \pi j d_{y} \sin \theta_{i r} \sin \phi_{i r}}, \ldots, e^{2 \pi j\left(N_{y}-1\right) d_{y} \sin \theta_{i r} \sin \phi_{i r}}\right]^{\mathrm{T}}
$$

Note that this definition of the steering vectors follows the notation in [144] where the array is defined to be in the $(x, y)$-plane. Hence, $\phi_{i r}$ is the angle of the $r^{\text {th }}$ ray for the $i^{\text {th }}$ user in the $(x, y)$-plane relative to the $x$-axis. The angle $\theta_{i r}$ is the angle of the $r^{\text {th }}$ ray for the $i^{\text {th }}$ user measured from the zenith direction (z-axis). Note that a similar diagram to that in Fig. (2.3) defines the elevation angle $\theta_{i r}$. With this definition, if the UPA is horizontally oriented then $\phi_{i r}$ is an azimuth angle and $\theta_{i r}$ is an elevation angle. However, for vertically located arrays, conventional $\theta_{i r}$ and $\phi_{i r}$ are defined relative to the $z$ and $x$ axes, respectively. The $k^{\text {th }}$ elements of $\mathbf{a}_{i r x}$ and $\mathbf{a}_{i r y}$ are denoted by $\mathbf{a}_{i r x k}$ and $\mathbf{a}_{\text {iryk }}$, respectively. In practice, each element has an active directional gain pattern, which attenuates the radiated power by an amount as a function of the steering direction. In order not to obfuscate the results and deviate our focus from the asymptotic massive MIMO properties, in this study, for all mathematical results, we assume that each element has an equal gain in all directions (i.e., omni-directional), irrespective of the location of the element in the array. However, we later briefly discuss the potential effects of nonisotropic antennas. 


\subsubsection{FP, Channel Hardening and Large System Potential}

Here, FP denotes asymptotic FP where $\mathbf{h}_{i}^{\mathrm{H}} \mathbf{h}_{j} / N \stackrel{\text { a.s. }}{\longrightarrow} 0$ as $N \rightarrow \infty$ [38]. Channel hardening refers to the property that $\mathbf{h}_{i}^{\mathrm{H}} \mathbf{h}_{i} / N \stackrel{\text { a.s. }}{\rightarrow} \beta_{i}$ as $N \rightarrow \infty$, which is equivalent in our case to the definition in [75]. Now, FP and channel hardening imply that the interference from one user to another vanishes relative to the signal power almost surely as $N \rightarrow \infty$. We extend this definition to the asymptotic regime where $N \rightarrow \infty, K \rightarrow \infty$ and $N / K \rightarrow \alpha$ (double-sided massive MIMO). Here, the equivalent question concerns whether the total interference power caused to user $i$ dominates the signal power. In order to investigate this behavior, we define

$$
\zeta_{\mathrm{LSP}}={ }^{4} \frac{\mathbb{E}\left[\left|\mathbf{h}_{i}^{\mathbf{H}} \mathbf{h}_{i}\right|^{2}\right]}{\sum_{j \neq i}^{K} \mathbb{E}\left[\left|\mathbf{h}_{i}^{\mathbf{H}} \mathbf{h}_{j}\right|^{2}\right]}=\frac{\mathbb{E}\left[\left|\mathbf{h}_{i}^{\mathbf{H}} \mathbf{h}_{i} / N\right|^{2}\right]}{\sum_{j \neq i}^{K} \mathbb{E}\left[\left|\mathbf{h}_{i}^{\mathbf{H}} \mathbf{h}_{j} / N\right|^{2}\right]}
$$

Now, $\zeta_{\text {LSP }}$ is a fundamental performance metric, measuring the ratio of the desired mean channel power to the total mean interference power. We say that LSP holds if $\zeta_{\text {LSP }}$ converges to a positive constant as $N \rightarrow \infty$ and $N / K \rightarrow$ $\alpha$. If channel hardening holds, then $\mathbf{h}_{i}^{\mathbf{H}} \mathbf{h}_{i} / N$ converges to a positive constant and hence, the numerator of (7.2) also converges to a positive constant. Even when channel hardening does not occur (see Sec. 7.3.1), the expectation in the numerator of (7.2) will converge to a constant.

If channel hardening holds then the numerator of (7.2) converges to a positive constant. Hence, LSP depends on the limiting behavior of the denominator of (7.2), namely $\mathbb{E}\left[\eta_{i}\right]$, where $\eta_{i}=\sum_{j \neq i}^{K}\left|\mathbf{h}_{i}^{\mathbf{H}} \mathbf{h}_{j} / N\right|^{2}$ is the total normalized interference. Note that the limiting regime used for LSP, which supports double-sided massive MIMO, is far more challenging than traditional massive MIMO. In practice, the number of users will never grow without bound

\footnotetext{
${ }^{4} \mathbb{E}\left[\left|\mathbf{h}_{i}^{\mathbf{H}} \mathbf{h}_{i}\right|^{2}\right]$ is the MRC signal power and $\sum_{j \neq i}^{K} \mathbb{E}\left[\left|\mathbf{h}_{i}^{\mathbf{H}} \mathbf{h}_{j}\right|^{2}\right]$ is the total MRC interference power.
} 
but the asymptotics are still useful in identifying the key properties of systems which are large in both $N$ and $K$.

\subsection{ULA: Channel Hardening, FP and LSP}

In this section, channel hardening, FP and LSP are considered for ray-based channel models for a ULA. For ease of notation, let $\mathbf{a}_{i r}$ be the steering vector for user $i$, path $r$, so that $\mathbf{a}_{i r}=\mathbf{a}\left(\phi_{i r}\right)$ and the $k^{\text {th }}$ element of $\mathbf{a}_{i r}$ is denoted $\mathbf{a}_{i r k}$.

\subsubsection{Channel Hardening}

Consider the term, $\mathbf{h}_{i}^{\mathbf{H}} \mathbf{h}_{i} / N$, for a ULA. We have

$$
\begin{aligned}
\frac{\mathbf{h}_{i}^{\mathbf{H}} \mathbf{h}_{i}}{N} & =\frac{1}{N} \sum_{r=1}^{P} \gamma_{i r}^{*} \mathbf{a}_{i r}^{\mathbf{H}} \sum_{s=1}^{P} \gamma_{i s} \mathbf{a}_{i s} \\
& =\sum_{r=1}^{P}\left|\gamma_{i r}\right|^{2}+\frac{1}{N} \sum_{r=1, r \neq s}^{P} \sum_{s=1}^{P} \gamma_{i r}^{*} \gamma_{i s} \mathbf{a}_{i r}^{\mathbf{H}} \mathbf{a}_{i s} \\
& =X_{i}+E_{i},
\end{aligned}
$$

where $X_{i}=\sum_{r=1}^{P}\left|\gamma_{i r}\right|^{2}$ is independent of $N$. Thus the limiting value depends entirely on $\lim _{N \rightarrow \infty} E_{i}$, which in turn depends on $\lim _{N \rightarrow \infty} \mathbf{a}_{i r}^{\mathbf{H}} \mathbf{a}_{i s} / N$, where $r \neq$ s. Now,

$$
\begin{aligned}
\left|\frac{\mathbf{a}_{i r}^{\mathbf{H}} \mathbf{a}_{i s}}{N}\right| & =\left|\frac{1}{N} \sum_{n=0}^{N-1} e^{-j 2 \pi d n \sin \phi_{i r}} e^{j 2 \pi d n \sin \phi_{i s}}\right| \\
& =\frac{1}{N}\left|\frac{\sin (N \iota / 2)}{\sin (\iota / 2)}\right| \stackrel{\text { a.s. }}{\longrightarrow} 0,
\end{aligned}
$$

where $\iota=2 \pi d\left[\sin \phi_{i s}-\sin \phi_{i r}\right]$, using simple results on geometric series. Almost sure convergence follows from the fact that convergence is guaranteed unless $\sin \phi_{i r}=\sin \phi_{i s}$, an event having probability zero for continuous an- 
gular variables ${ }^{5}$. Thus, we have $\mathbf{h}_{i}^{\mathbf{H}} \mathbf{h}_{i} / N \stackrel{\text { a.s. }}{\longrightarrow} X_{i}$ as $N \rightarrow \infty$. Note that for random phase models, $X_{i}=\beta_{i}$ and traditional channel hardening occurs where $\mathbf{h}_{i}^{\mathbf{H}} \mathbf{h}_{i} / N \stackrel{\text { a.s. }}{\longrightarrow} \beta_{i}$, a deterministic limit. In contrast, for complex Gaussian models, $\left|\gamma_{i r}\right|^{2}=\beta_{i r}\left|u_{i r}\right|^{2}$, which gives a random limit, as $X_{i}=\sum_{r=1}^{P}\left|\gamma_{i r}\right|^{2}$ is a weighted sum of exponential variables. Hence, we see that the existence of channel hardening depends on the nature of the model for the ray coefficients. Note that the channel hardening analysis in [146] was for arbitrary array topologies but relied on uniform angles.

\subsubsection{FP (Single-Sided Massive MIMO)}

In terms of FP, results are simple following the same methodology as for channel hardening. First, we write

$$
\frac{\mathbf{h}_{i}^{\mathbf{H}} \mathbf{h}_{j}}{N}=\frac{1}{N} \sum_{r=1}^{P} \sum_{s=1}^{P} \gamma_{i r}^{*} \gamma_{j s} \mathbf{a}_{i r}^{\mathbf{H}} \mathbf{a}_{j s}
$$

and then we use (7.4) to show that

$$
\left|\frac{\mathbf{a}_{i r}^{\mathbf{H}} \mathbf{a}_{j s}}{N}\right|=\frac{1}{N}\left|\frac{\sin (N \tau / 2)}{\sin (\tau / 2)}\right| \stackrel{\text { a.s. }}{\longrightarrow} 0,
$$

as $N \rightarrow \infty$, where $\tau=2 \pi d\left[\sin \phi_{j s}-\sin \phi_{i r}\right]$. Hence, FP is proven very simply for all ray-based models where $\sin \phi_{i r}=\sin \phi_{i s}$ has probability zero. A simple condition for this to hold is that the angles are continuous random variables, a property held by all models proposed ${ }^{6}$. Therefore, FP, the key property en-

\footnotetext{
${ }^{5}$ Our model (including that in [35] and 3GPP, 3GPP TR 21.915 V15.0.0, http://www.3gpp.org/) and all other such ray-based models [48] assume a finite number of unique angles for the rays. The probability of two angles being identical is zero if the angle distributions are continuous. This follows as $P(X=Y)=0$ for all distinct, continuous random variables $X$ and $Y$ [150, p. 76]. Our analysis is limited to this scenario and does not cover possible models which could explain a "single" ray as a continuum of rays over a small angular width.

${ }^{6}$ This was demonstrated in [144], but only for the case of a uniform distribution. In contrast, the result in (7.6) is general.
} 
abling single-sided massive $\mathrm{MIMO}$, holds for all ray distributions considered to date, such as uniform, wrapped Gaussian, and Laplacian.

\subsubsection{Large System Potential (Double-Sided Massive MIMO)}

In this section, we analyze the LSP of ray-based channels in the limiting regime where $K \rightarrow \infty, N \rightarrow \infty$ and $N / K \rightarrow \alpha$.

\section{Ray-based Models}

The ratio $\zeta_{\text {LSP }}$ in (7.2) has a numerator satisfying $\mathbb{E}\left[\left|\mathbf{h}_{i}^{\mathrm{H}} \mathbf{h}_{i}\right|^{2} / N^{2}\right] \stackrel{\text { a.s. }}{\longrightarrow} \mathbb{E}\left[X_{i}^{2}\right]$ from Sec. 7.3.1 and $\mathbb{E}\left[X_{i}^{2}\right]$ is finite. Hence, LSP depends on the asymptotic properties of the denominator, $\mathbb{E}\left[\eta_{i}\right]$. Here, we write,

$$
\begin{aligned}
\mathbb{E}\left[\eta_{i}\right] & =\mathbb{E}\left[\frac{1}{N} \sum_{j=1, j \neq i}^{K} \frac{1}{N}\left|\sum_{r=1}^{P} \sum_{s=1}^{P} \gamma_{i r}^{*} \gamma_{j s} \mathbf{a}_{i r}^{\mathrm{H}} \mathbf{a}_{j s}\right|^{2}\right] \\
& =\frac{1}{N} \sum_{j=1, j \neq i}^{K} \sum_{r=1}^{P} \sum_{s=1}^{P} \mathbb{E}\left[\left|\gamma_{i r}\right|^{2}\right] \mathbb{E}\left[\left|\gamma_{j s}\right|^{2}\right] \frac{1}{N} \mathbb{E}\left[\left|\mathbf{a}_{i r}^{\mathrm{H}} \mathbf{a}_{j s}\right|^{2}\right] \\
& =\beta_{i}\left(\frac{\sum_{j=1, j \neq i}^{K} \beta_{j}}{N}\right) \mu_{\mathrm{ULA}},
\end{aligned}
$$

using the basic properties of the $\gamma_{i r}$ terms and the notation

$$
\begin{aligned}
\mu_{\mathrm{ULA}} & \triangleq \frac{1}{N} \mathbb{E}\left[\left|\mathbf{a}_{i r}^{\mathrm{H}} \mathbf{a}_{j s}\right|^{2}\right] \\
& =\frac{1}{N} \sum_{n=0}^{N-1} \sum_{m=0}^{N-1} \mathbb{E}\left[e^{j 2 \pi d(m-n) \sin \phi_{i r}}\right] \mathbb{E}\left[e^{j 2 \pi d(n-m) \sin \phi_{j s}}\right] \\
& =\frac{1}{N} \sum_{n=0}^{N-1} \sum_{m=0}^{N-1}\left|\mathbb{E}\left[e^{j 2 \pi d(m-n) \sin \phi_{i r}}\right]\right|^{2}
\end{aligned}
$$

This relies on the fact that the scaling factors are independent and azimuth angles are i.i.d random variables. Hence, the steering vectors in $\mu_{\mathrm{ULA}}$ are two generic but independent vectors and the subscripts $i r$ and $j s$ are not strictly 
necessary. Now, we set $\hat{\phi}_{i r}=2 \pi d \sin \phi_{i r}$, and rewrite (7.8) as

$$
\begin{aligned}
\mu_{\mathrm{ULA}} & =\frac{1}{N}\left(\sum_{n=0}^{N-1} 1+\sum_{m, n=0, m \neq n}^{N-1} \sum^{N}\left|\mathbb{E}\left[e^{j 2 \pi d(m-n) \sin \phi_{i r}}\right]\right|^{2}\right) \\
& =1+\frac{2}{N} \sum_{s=1}^{N-1}(N-s)\left|\mathbb{E}\left[e^{-j 2 \pi d s \sin \phi_{i r}}\right]\right|^{2} \\
& =1+2 \sum_{s=1}^{N-1}\left(1-\frac{s}{N}\right)\left|\mathbb{E}\left[e^{-j s \hat{\phi}_{i r}}\right]\right|^{2} .
\end{aligned}
$$

In a typical drop of random locations for the users, the strong law of large numbers ensures that $\sum_{j=1}^{K} \beta_{j} / K$ converges to $\bar{\beta}$ as $K \rightarrow \infty$, where $\bar{\beta}$ is a finite mean power. Hence, we have $\lim _{N \rightarrow \infty} \mathbb{E}\left[\eta_{i}\right]=\left(\beta_{i} \bar{\beta} / \alpha\right) \lim _{N \rightarrow \infty}\left\{\mu_{\mathrm{ULA}}\right\}$. Hence, the asymptotic behavior of $\mathbb{E}\left[\eta_{i}\right]$ depends on $\mu_{\mathrm{ULA}}$ which in turn depends on how quickly $\left|\mathbb{E}\left[e^{-j s \hat{\phi}_{i r}}\right]\right|^{2}$ decays. In the following theorem we present a general answer to this question.

Theorem 1. The term $\mathbb{E}\left[e^{-j s \hat{\phi}_{i r}}\right]$ decays as $s^{-\frac{1}{2}}$ as $s \rightarrow \infty$ with the asymptotic representation:

$$
\mathbb{E}\left[e^{-j s \hat{\phi}_{i r}}\right] \sim \frac{1}{\sqrt{d s}}\left(f_{\phi}\left(-\frac{\pi}{2}\right) e^{j\left(2 \pi d s-\frac{\pi}{4}\right)}+f_{\phi}\left(\frac{\pi}{2}\right) e^{-j\left(2 \pi d s-\frac{\pi}{4}\right)}\right)
$$

where $f_{\phi}(\cdot)$ is the probability density function (PDF) of $\phi_{i r}$.

Proof. The proof is given in Appendix 9.1.

\section{Implications of Theorem 1}

Equation (7.10) in Theorem 1 is a remarkable result with a simple and intuitive interpretation, wide generality and important implications for massive MIMO:

- In terms of generality, (7.10) only requires the angular PDF, $f_{\phi}(\cdot)$, not to have singularities which are worse than $O\left(x^{-1 / 2}\right)$ at $x=0$. This covers 
all proposed models. Certainly, all proposed models thus far are continuous, so this condition is easily satisfied.

- Interpreting (7.10) we see that if the end-fire direction has no energy, $f_{\phi}( \pm \pi / 2)=0$, then $\mathbb{E}\left[e^{-j s \hat{\phi}_{i r}}\right]=0$. Alternatively, if some end-fire radiation occurs then $f_{\phi}( \pm \pi / 2)>0$ and $\mathbb{E}\left[e^{-j s \hat{\phi}_{i r}}\right]=O\left(s^{-1 / 2}\right)$. From the above, it follows that if there is no end-fire radiation, $\mu_{\mathrm{ULA}}$ is finite and the mean interference cannot dominate the mean power of the desired channel.

- Further, if there is end-fire radiation, then LSP does not hold as $\mu_{\mathrm{ULA}} \rightarrow$ $\infty$. This conclusion holds by inspection of (7.9). When $\mathbb{E}\left[e^{-j s \hat{\phi}_{i r}}\right]$ is $O\left(s^{-1 / 2}\right)$, then $\sum_{s=1}^{N}\left|\mathbb{E}\left[e^{-j s \hat{\phi}_{i r}}\right]\right|^{2}$ is $O(\log N)$ using well known properties of the series $\sum_{s=1}^{N} \frac{1}{s}$. Also, $\sum_{s=1}^{N} \frac{s}{N}\left|\mathbb{E}\left[e^{-j s \hat{\phi}_{i r}}\right]\right|^{2}$ is finite, so that $\mu_{\mathrm{ULA}}$ grows to infinity, but at a very slow logarithmic rate. Note that this interference growth can be described as critical as $\mathbb{E}\left[e^{-j s \hat{\phi}_{i r}}\right]$ decays at exactly the critical rate $\left(s^{-1 / 2}\right)$ required for logarithmic growth. Any reduction at all in the decay rate would deliver finite interference and therefore would enable LSP to hold.

- The importance of the end-fire direction can be understood in the following way. For a ULA, it is not the proximity of two incoming ray angles that drives the interference, but the difference in the sines of the angles (see $\tau$ in (7.6)). For angles close to broadside the difference in sines is largest and for angles near end-fire the difference is smallest, resulting in greater interference.

- We note that LSP does hold for i.i.d. Rayleigh fading channels. To see this we use the results in [7] to give $\zeta_{\text {LSP }}=\beta_{i} \bar{\beta} / \alpha$. Hence, ray-based models differ from classical statistical channel models in this regard. 
- Overall, the result in (7.10) is extremely positive for double-sided massive MIMO. We have shown that in the challenging scenario where both $K$ and $N$ grow large, the interference, relative to the power of the desired channel, grows very slowly (logarithmically). Also, the scaling of this growth factor is very small, since a large amount of end-fire radiation is unlikely, and practical deployments typically employ patch elements which have average look angles on the order of $+/-45^{\circ}$ (designed according to the downtilt angle of the array as well as the environment) [151]. This substantially attenuates the end-fire (or near by) radiation since the patterns create explicit nulls to reject the incoming wavefronts in those directions. Although not shown in the thesis, following the approach in [35] the effect of antenna patterns can be modelled as a multiplicative scaling of the ray powers which varies according to the angle. This causes the typical end-fire reduction.

Given the power of these results, it is useful to validate the conclusions with some special cases. Note that although uniform and von-Mises (VM) angular distributions are not derived from measurements in real environments they are useful, both for validating Theorem 1 and providing exact asymptotics which are not perturbed by simulation error.

\section{Special Cases: Uniform Distribution}

When $\phi_{i r} \sim U[0,2 \pi], f_{\phi}(x)=\frac{1}{2 \pi}$ for $-\pi \leq x \leq \pi$ and (7.10) becomes

$$
\mathbb{E}\left[e^{-j s \hat{\phi}_{i r}}\right] \sim \frac{1}{\pi \sqrt{d s}} \cos \left(2 \pi d s-\frac{\pi}{4}\right)
$$

This limiting value is verified in the uniform case where the exact solution is known as $\mathbb{E}\left[e^{-j s \hat{\phi}_{i r}}\right]=\mathbb{E}\left[e^{-j s 2 \pi d \sin \phi_{i r}}\right]=J_{0}(2 \pi d s)[132$, p. 375]. For large values of $s, J_{0}(2 \pi d s) \sim \cos \left(2 \pi d s-\frac{\pi}{4}\right) / \pi \sqrt{d s}$ [132, p. 364], which agrees with (7.10). 
Hence, the general asymptotic analysis in (7.10) is supported and the exact value of $\mathbb{E}\left[e^{-j s \hat{\phi}_{i r}}\right]$ can be used in (7.9) to give the exact value of $\mu_{\mathrm{ULA}}$.

\section{Special Cases: Von-Mises Distribution}

The VM distribution has also been used in angular modeling [152] and has the PDF given by

$$
f_{\phi}(x)=\frac{e^{\kappa \cos (x-\mu)}}{2 \pi I_{0}(\kappa)},-\pi \leq x \leq \pi,
$$

where $\mu$ is a measure of location and $\kappa$ is a measure of concentration. Substituting the VM PDF into (7.10) we obtain

$$
\mathbb{E}\left[e^{-j s \hat{\phi}_{i r}}\right] \sim \frac{e^{j 2 \pi d s}}{\sqrt{d s}}\left(\frac{e^{\kappa \cos \left(-\frac{\pi}{2}-\mu\right)}}{2 \pi I_{0}(\kappa)} e^{-j \frac{\pi}{4}}+\frac{e^{\kappa \cos \left(\frac{\pi}{2}-\mu\right)}}{2 \pi I_{0}(\kappa)} e^{j \frac{\pi}{4}}\right)
$$

The exact solution can be found by integration, giving

$$
\mathbb{E}\left[e^{-j s \hat{\phi}_{i r}}\right]=\frac{I_{0}\left(\sqrt{\kappa^{2} \cos ^{2}(\mu)+(\kappa \sin (\mu)-j 2 \pi d s)^{2}}\right)}{I_{0}(\kappa)} .
$$

Further analysis shows that (7.14) is asymptotically equal to (7.13). This is briefly explained as follows. For large $q$, the argument of $I_{0}(\cdot)$ in the numerator of (7.14) is approximately $\kappa \sin (\mu)-j 2 \pi d q$. Then we use the large argument approximation of $I_{0}(\kappa \sin (\mu)-j 2 \pi d q)$ [132, p. 364] and simplify to give (7.13). Hence, for the VM case also, we have verified (7.10) and given an exact solution for $\mathbb{E}\left[e^{-j q \hat{\phi}_{i r}}\right]$.

Hence, for the VM case also, we have verified (7.10) and given an exact solution for $\mathbb{E}\left[e^{-j s \hat{\phi}_{i r}}\right]$. 


\subsubsection{Avoiding Interference Growth}

Since the logarithmic interference growth predicted by Theorem 1 is critical, it can be removed by simple methods. For example, LSP can be assured by trivial scheduling methods based on user separation. This is shown in the following.

Expanding $\eta_{i}=\sum_{j \neq i}^{K}\left|\mathbf{h}_{i}^{\mathbf{H}} \mathbf{h}_{j} / N\right|^{2}$ in terms of the steering vectors gives

$$
\eta_{i}=\sum_{j \neq i} \frac{1}{N^{2}} \sum_{r, s} \sum_{r^{\prime}, s^{\prime}} \gamma_{i r}^{*} \gamma_{j s} \gamma_{i r^{\prime}} \gamma_{j s^{\prime}}^{*} \mathbf{a}_{i r}^{\mathrm{H}} \mathbf{a}_{i s} \mathbf{a}_{j s^{\prime}}^{\mathbf{H}} \mathbf{a}_{i r^{\prime}} .
$$

From (7.4), $\eta_{i}$ can be rewritten as

$$
\eta_{i}=\sum_{j \neq i} \sum_{r, s, r^{\prime}, s^{\prime}} \Gamma_{i j r s r^{\prime} s^{\prime}} \frac{1}{N}\left|\frac{\sin \left(N \tau_{1} / 2\right)}{\sin \left(\tau_{1} / 2\right)}\right| \frac{1}{N}\left|\frac{\sin \left(N \tau_{2} / 2\right)}{\sin \left(\tau_{2} / 2\right)}\right|
$$

where $\sum_{r, s, r^{\prime}, s^{\prime}}=\sum_{r, s} \sum_{r^{\prime}, s^{\prime}}$ and $\Gamma_{i j r s r^{\prime} s^{\prime}}=\gamma_{i r}^{*} \gamma_{j s} \gamma_{i r^{\prime}} \gamma_{j s^{\prime}}^{*} \tau_{1}=2 \pi d\left[\sin \phi_{j s}-\right.$ $\left.\sin \phi_{i r}\right]$ and $\tau_{2}=2 \pi d\left[\sin \phi_{i r^{\prime}}-\sin \phi_{j s^{\prime}}\right]$. Note that $\sum_{r, s, r^{\prime}, s^{\prime}} \Gamma_{i j r s r^{\prime} s^{\prime}}$ is finite and for $\tau_{i} \neq 0,\left|\frac{\sin \left(N \tau_{i} / 2\right)}{\sin \left(\tau_{i} / 2\right)}\right|$ is bounded for $i=1,2$. Hence, for finite $K, \eta_{i} \rightarrow 0$ for $\tau_{1} \neq 0, \tau_{2} \neq 0$ as the number of terms in the sum is equal to $K-1$ but the summand is $O\left(N^{-2}\right)$. Since $P\left(\tau_{1}=0\right)=P\left(\tau_{2}=0\right)=0$ for continuous angular variables, it follows that $\eta_{i} \stackrel{\text { a.s. }}{\longrightarrow} 0$ and LSP holds.

Next, consider the asymptotic case where the number of users is growing but users are only scheduled together if the sines of their ray angles are separated by more than a given fixed protection level, $\epsilon>0$. Hence, $\left|\tau_{i}\right|>2 \pi d \epsilon$ for $i=1,2$. Using this inequality in (7.16) gives

$$
\eta_{i} \leq \frac{1}{N^{2} \sin ^{2}(\pi d \epsilon)} \sum_{j \neq i} \sum_{r, s, r^{\prime}, s^{\prime}} \Gamma_{i j r s r^{\prime} s^{\prime}}
$$

Since the right hand side of (7.17) converges to zero as $N \rightarrow \infty$ it follows that $\eta_{i} \stackrel{\text { a.s. }}{\longrightarrow} 0$ and LSP holds. 


\subsection{UPA: Channel Hardening, FP and LSP}

In this section, we extend the ULA results on channel hardening, FP and LSP to a UPA. Throughout the work on LSP for a UPA we assume that $N_{x} \rightarrow \infty$ and $N_{y} \rightarrow \infty$ as $N \rightarrow \infty$.

\subsubsection{Channel Hardening}

The channel hardening results presented in (7.3) for a ULA remain valid for any array structure. Hence, $\mathbf{h}_{i}^{\mathbf{H}} \mathbf{h}_{i} / N \stackrel{\text { a.s. }}{\longrightarrow} X_{i}$ if $\mathbf{a}_{i r}^{\mathbf{H}} \mathbf{a}_{i s} \stackrel{\text { a.s. }}{\longrightarrow} 0$. For the UPA we have

$$
\begin{aligned}
\frac{\mathbf{a}_{i r}^{\mathbf{H}} \mathbf{a}_{i s}}{N} & =\frac{1}{N}\left(\mathbf{a}_{i r x} \otimes \mathbf{a}_{i r y}\right)^{\mathbf{H}}\left(\mathbf{a}_{i s x} \otimes \mathbf{a}_{i s y}\right) \\
& =\frac{1}{N}\left(\mathbf{a}_{i r x}^{\mathbf{H}} \mathbf{a}_{i s x}\right)\left(\mathbf{a}_{i r y}^{\mathbf{H}} \mathbf{a}_{i s y}\right),
\end{aligned}
$$

from basic properties of Kronecker products. Now, the cross products $\mathbf{a}_{i r x}^{\mathbf{H}} \mathbf{a}_{i s x}$ and $\mathbf{a}_{i r y}^{\mathbf{H}} \mathbf{a}_{i s y}$ have a similar form to the ULA. Hence, from (7.3) we can deduce that

$$
\left|\frac{\mathbf{a}_{i r}^{\mathbf{H}} \mathbf{a}_{i s}}{N}\right|=\frac{1}{N_{x}}\left|\frac{\sin \left(N_{x} \tau_{x} / 2\right)}{\sin \left(\tau_{x} / 2\right)}\right| \frac{1}{N_{y}}\left|\frac{\sin \left(N_{y} \tau_{y} / 2\right)}{\sin \left(\tau_{y} / 2\right)}\right|,
$$

where $\tau_{x}=2 \pi d_{x}\left(\sin \theta_{i s} \cos \phi_{i s}-\sin \theta_{i r} \cos \phi_{i r}\right)$ and $\tau_{y}=2 \pi d_{y}\left(\sin \theta_{i s} \sin \phi_{i s}-\right.$ $\left.\sin \theta_{i r} \sin \phi_{i r}\right)$. As in Sec. 7.3.1, $\mathbf{a}_{i r}^{\mathbf{H}} \mathbf{a}_{i s} / N$ converges to zero unless $\tau_{x}=0$ or $\tau_{y}=$ 0 , an event having probability zero. Hence $\mathbf{a}_{i r}^{\mathbf{H}} \mathbf{a}_{i s} / N \stackrel{\text { a.s. }}{\longrightarrow} 0$ and $\mathbf{h}_{i}^{\mathbf{H}} \mathbf{h}_{i} / N \stackrel{\text { a.s. }}{\longrightarrow} X_{i}$. 


\subsubsection{FP (Single-Sided Massive MIMO)}

The ULA results in (7.5) and (7.6) show that for any array, FP occurs if $\frac{\mathbf{a}_{i r}^{\mathbf{H}} \mathbf{a}_{j s}}{N} \stackrel{\text { a.s. }}{\longrightarrow}$ 0 as $N \rightarrow \infty$. Following the same calculation as in (7.19) gives

$$
\left|\frac{\mathbf{a}_{i r}^{\mathbf{H}} \mathbf{a}_{j s}}{N}\right|=\frac{1}{N_{x}}\left|\frac{\sin \left(N_{x} \tau_{x} / 2\right)}{\sin \left(\tau_{x} / 2\right)}\right| \frac{1}{N_{y}}\left|\frac{\sin \left(N_{y} \tau_{y} / 2\right)}{\sin \left(\tau_{y} / 2\right)}\right|,
$$

where $\tau_{x}=2 \pi d_{x}\left(\sin \theta_{j s} \cos \phi_{j s}-\sin \theta_{i r} \cos \phi_{i r}\right)$ and $\tau_{y}=2 \pi d_{y}\left(\sin \theta_{j s} \sin \phi_{j s}-\right.$ $\left.\sin \theta_{i r} \sin \phi_{i r}\right)$. Since $P\left(\tau_{x}=0\right)=P\left(\tau_{y}=0\right)=0$ for continuous angular variables, it follows that $\eta_{i} \stackrel{\text { a.s. }}{\longrightarrow} 0$ and FP holds.

\subsubsection{Large System Potential (Double-Sided Massive MIMO)}

The existence of LSP for a UPA depends on whether the expression $\mu_{\mathrm{UPA}}=$ $\frac{1}{N} \mathbb{E}\left[\left|\mathbf{a}_{i r}^{\mathrm{H}} \mathbf{a}_{j s}\right|^{2}\right]$ converges or not. This is analyzed in Theorem 2.

Theorem 2. The term $\mu_{U P A}$ for a UPA antenna structure grows logarithmically with the following representation:

$$
\mu_{\text {UPA }}=\sum_{v=1-N_{x}}^{N_{x}-1} \sum_{w=1-N_{y}}^{N_{y}-1}\left(1-\frac{|v|}{N_{x}}\right)\left(1-\frac{|w|}{N_{y}}\right)\left|M_{v, w}\right|^{2},
$$

where $M_{v, w}=\mathbb{E}\left[e^{-j 2 \pi \nu_{v, w} \sin (\theta) \cos \left(\phi-\Delta_{v, w}\right)}\right], \nu_{v, w}=\sqrt{v^{2} d_{x}^{2}+w^{2} d_{y}^{2}}$

and $\Delta_{v, w}=\tan ^{-1}\left(\frac{w d_{y}}{v d_{x}}\right)$.

Proof. The proof is given in Appendix 9.2.

\section{Implications of Theorem 2}

As in the ULA case, Appendix 9.2 shows that the interference growth is critical and any reduction in the rate of interference accumulation will lead to LSP holding. The result holds for any continuous angular distribution so it is 
extremely general. Note that a ULA would normally aim to null the end-fire direction (see (7.10)) and perfect nulling would avoid the interference growth. In contrast, a similar argument using (9.19) shows that $f_{\theta}(x)$ must equal zero for $x$ in $\{-\pi / 2,0, \pi / 2\}$ in order to avoid interference growth. For a vertical UPA, to null the broadside direction is clearly unsuitable as it requires nulling the dominant azimuth plane $(\theta=0)$. In general for all types of UPA, a more symmetric structure means that there are no sets of special angles which avoid interference growth and for which the radiation is unwanted. Since the proof is complex, it is instructive to look at the spherical uniform case where $\mu_{\mathrm{UPA}}$ can be derived and shown to grow logarithmically as in Theorem 2 . This is shown in the following.

\section{Special Case: Uniform Distribution}

We derive an equation for $\mu_{\mathrm{UPA}}$ in the simplest uniform case, where the azimuth angle is $\phi_{i r} \sim U[0,2 \pi]$ and the elevation angle is $\theta_{i r} \sim U\left[-\frac{\pi}{2}, \frac{\pi}{2}\right]$. In this scenario, the following result applies.

Result 1. The value of $\mu_{U P A}$ for spherically uniform angles is given by

$$
\mu_{U P A}=\frac{1}{N} \sum_{r_{x}=1}^{N_{x}} \sum_{r_{y}=1}^{N_{y}} \sum_{s_{x}=1}^{N_{x}} \sum_{s_{y}=1}^{N_{y}} J_{0}^{4}\left(\pi \sqrt{d_{y}^{2}\left(r_{y}-s_{y}\right)^{2}+d_{x}^{2}\left(r_{x}-s_{x}\right)^{2}}\right)
$$

where the proof is given in Appendix 9.3. In Appendix 9.4, this result is used to demonstrate the logarithmic growth of $\mu_{\mathrm{UPA}}$.

\section{Avoiding Interference Growth}

As for the ULA, interference growth can be avoided by using finite $K$ or simple scheduling. This is shown in the following. For a UPA, substituting the 
associated steering vectors into (7.15) gives

$$
\eta_{i}=\sum_{j \neq i} \frac{1}{N^{2}} \sum_{r, s, r^{\prime}, s^{\prime}} \Gamma_{i j r s r^{\prime} s^{\prime}} \mathbf{a}_{i r x}^{\mathrm{H}} \mathbf{a}_{i s x} \mathbf{a}_{i r y}^{\mathrm{H}} \mathbf{a}_{i s y} \mathbf{a}_{j s^{\prime} x}^{\mathbf{H}} \mathbf{a}_{i r^{\prime} x} \mathbf{a}_{j s^{\prime} y}^{\mathbf{H}} \mathbf{a}_{i r^{\prime} y} .
$$

As in (7.19), all of the four cross products of steering vectors in $x$ and $y$ domains have representations as ratios of sine functions. Hence,

$\eta_{i}=\sum_{j \neq i} \frac{1}{N^{2}} \sum_{r, s, r^{\prime}, s^{\prime}} \Gamma_{i j r s r^{\prime} s^{\prime}}\left|\frac{\sin \left(N_{x} \tau_{x} / 2\right)}{\sin \left(\tau_{x} / 2\right)}\right|\left|\frac{\sin \left(N_{y} \tau_{y} / 2\right)}{\sin \left(\tau_{y} / 2\right)}\right|\left|\frac{\sin \left(N_{x} \tau_{x}^{\prime} / 2\right)}{\sin \left(\tau_{x}^{\prime} / 2\right)}\right|\left|\frac{\sin \left(N_{y} \tau_{y}^{\prime} / 2\right)}{\sin \left(\tau_{y}^{\prime} / 2\right)}\right|$

where $\tau_{x}$ and $\tau_{y}$ are defined in Sec. 7.4.2, $\tau_{x}^{\prime}=2 \pi d_{x}\left(\sin \theta_{i s^{\prime}} \cos \phi_{i s^{\prime}}-\sin \theta_{i r^{\prime}} \cos \phi_{i r^{\prime}}\right)$ and $\tau_{y}^{\prime}=2 \pi d_{y}\left(\sin \theta_{j s^{\prime}} \sin \phi_{j s^{\prime}}-\sin \theta_{j r^{\prime}} \sin \phi_{j r^{\prime}}\right)$. In the asymptotic case where $K \rightarrow \infty$, consider scheduling using a similar protection threshold as used in Sec. 7.3.4 where users are only selected if $\min \left(\tau_{x}, \tau_{y}, \tau_{x}^{\prime}, \tau_{y}^{\prime}\right)>2 \pi d \epsilon$. With this approach, we see that

$$
\eta_{i}<\sum_{j \neq i}^{K} \frac{1}{N^{2} \sin ^{4}(\pi d \epsilon)} \sum_{r, s, r^{\prime}, s^{\prime}} \Gamma_{i j r s r^{\prime} s^{\prime}}
$$

Since the right hand side of (7.24) converges to zero as $N \rightarrow \infty$ it follows that $\eta_{i} \stackrel{\text { a.s. }}{\longrightarrow} 0$ and LSP holds.

\subsection{Numerical Results}

In Fig. 7.1 we demonstrate the channel hardening and FP results for the ULA discussed in Sec. 7.3 for $K=2$ and an increasing number of antennas. We adopt the non-line-of-sight (NLOS) 3GPP angular and cluster parameters in [35]. The number of clusters is $C=20$, and the number of subpaths per cluster is $L=20$. Referring to the channel model in (7.1), $P=C L$. Each subpath angle of arrival (AoA) is modeled by a central cluster angle having 
a Gaussian distribution (zero mean and a standard deviation of $76.5^{\circ}$ ) plus a subray offset angle which is Laplacian with a standard deviation of $15^{\circ}$. We assume $\beta_{1}=\beta_{2}=1$ and subrays having equal powers ${ }^{7}$. From the upper plot of Fig. 7.1, we can see that the normalized power in the desired channel, $S=\left|\mathbf{h}_{i}^{\mathbf{H}} \mathbf{h}_{i}\right| / N \approx 1$ for large numbers of antennas. Similarly, the lower plot shows the mean of interference term, $I=\left|\mathbf{h}_{i}^{\mathbf{H}} \mathbf{h}_{j}\right| / N$ decreasing to zero as $N \rightarrow \infty$. Note that Fig. 7.1 plots $\mathbb{E}[I]$ for both the ray-based model (via simulation) and i.i.d. Rayleigh fading (via analysis) so that the variations do not obscure the trend. As expected, the convergence to FP is slower for the ray-based model but the initial rate of convergence is similar for both channels. Hence, both channel hardening and FP are shown to occur for a typical parameter set as predicted by the analysis. Fig. 7.1 shows channel hardening and FP occurring for a clustered channel model with wrapped Gaussian central cluster angles and Laplacian offsets. This numerical example is useful as it verifies the analysis for a commonly used ray-based model structure. The analysis goes much further and proves the existence/non-existence of channel hardening and the existence of FP for all ray-based of the form in (7.1) for a comprehensive range of ray distributions. These observations are in line with the channel hardening measurements reported in [153].

In Fig. 7.2, we show that the power of the desired channel will either converge to a constant or a random variable, verifying the analysis in Sec. 7.3.1. We assume the same model as in Fig. 7.1 but with two possibilities for the ray coefficients, $\gamma_{i r}$. The Akdeniz model [35] uses a complex Gaussian variable for $\gamma_{i r}$, while the 3GPP model [35] uses a random phase. As shown in Fig. 7.2, as the number of antennas grows, the cumulative distribution function (CDF) of the normalized desired channel power, $S=\mathbf{h}_{i}^{\mathbf{H}} \mathbf{h}_{i} / N$, with the

\footnotetext{
${ }^{7}$ Equal ray powers are adopted for simplicity in Fig. 7.1 and Fig. 7.2 for initial verification of the FP and channel hardening results, $\beta_{i r}=1 / C L$, and phases are uniformly distributed, $\phi_{\text {ir }} \sim U[0,2 \pi]$.
} 

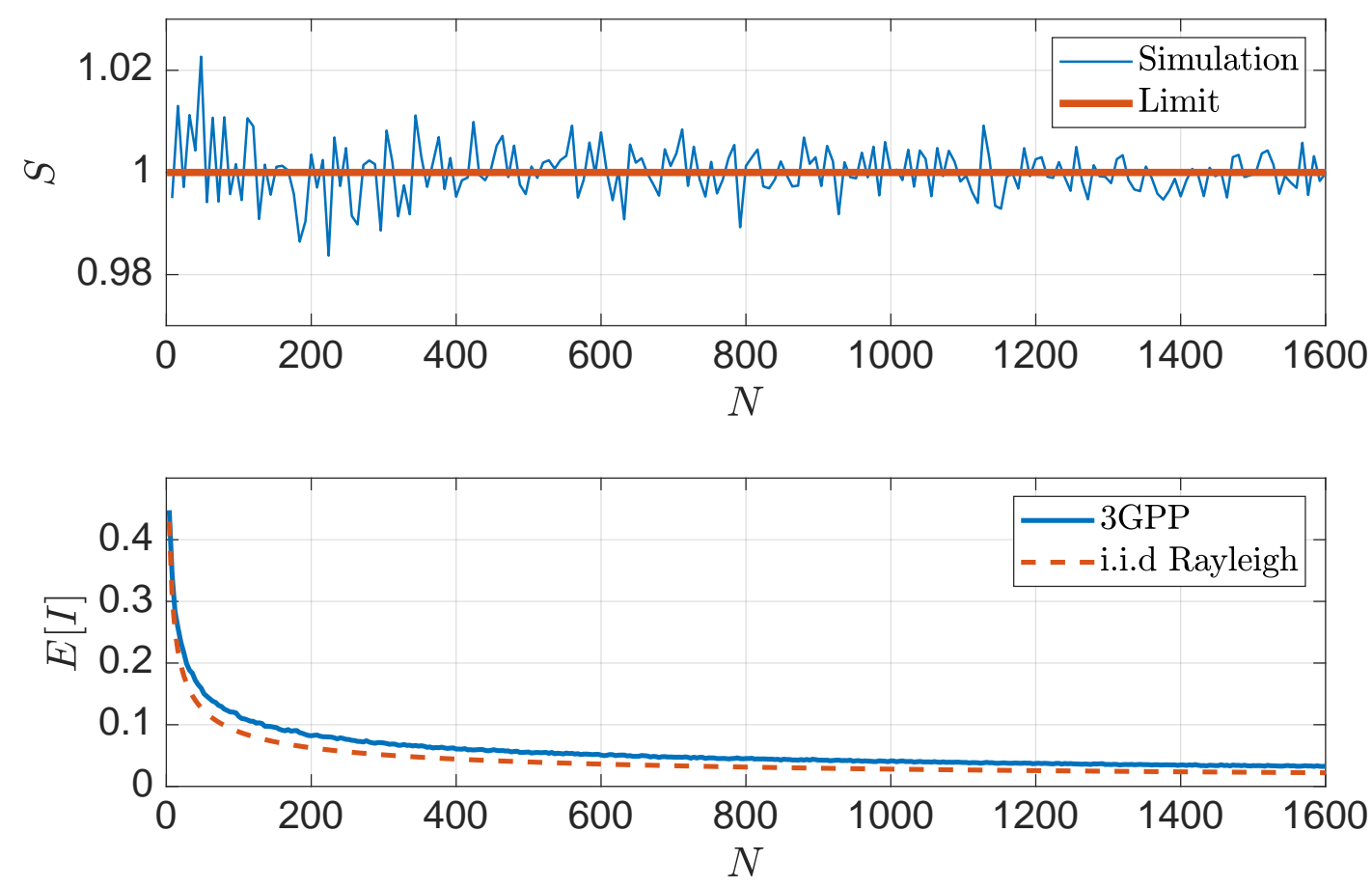

Figure 7.1: Channel hardening and FP (3GPP angular parameters).

Akdeniz model remains almost the same, indicating convergence to a random variable. In contrast, with the random phase model of 3GPP the CDF converges to a step function indicating that $S$ converges to a constant. Hence, as shown in Sec. 7.3.1, channel hardening can occur for ray-based models but this depends on the models employed for the ray coefficients.

In Fig. 7.3, we show both the simulated and analytical results for $\mu_{\mathrm{ULA}}$ with uniform and VM distributions using the results in Sec. 7.3.3. We also show simulated values of $\mu_{\mathrm{ULA}}$ by adopting the angular parameters of the 3GPP model in [35] as in Fig. 7.1. The number of antennas and users are growing at the same ratio $N / K=\alpha=2$, while $\phi_{i r} \sim U[0,2 \pi]$ for the uniform model and $\kappa=4.23$ (for $30^{\circ}$ angle spread) and $\mu=0$ for VM. From Fig. 7.3 we can see that the analysis agrees well with simulation for both uniform and VM models. We also notice that the growth rates of $\mu_{\mathrm{ULA}}$ are different for all three models, due to the differences in the AoA distributions. In the following figure, we 


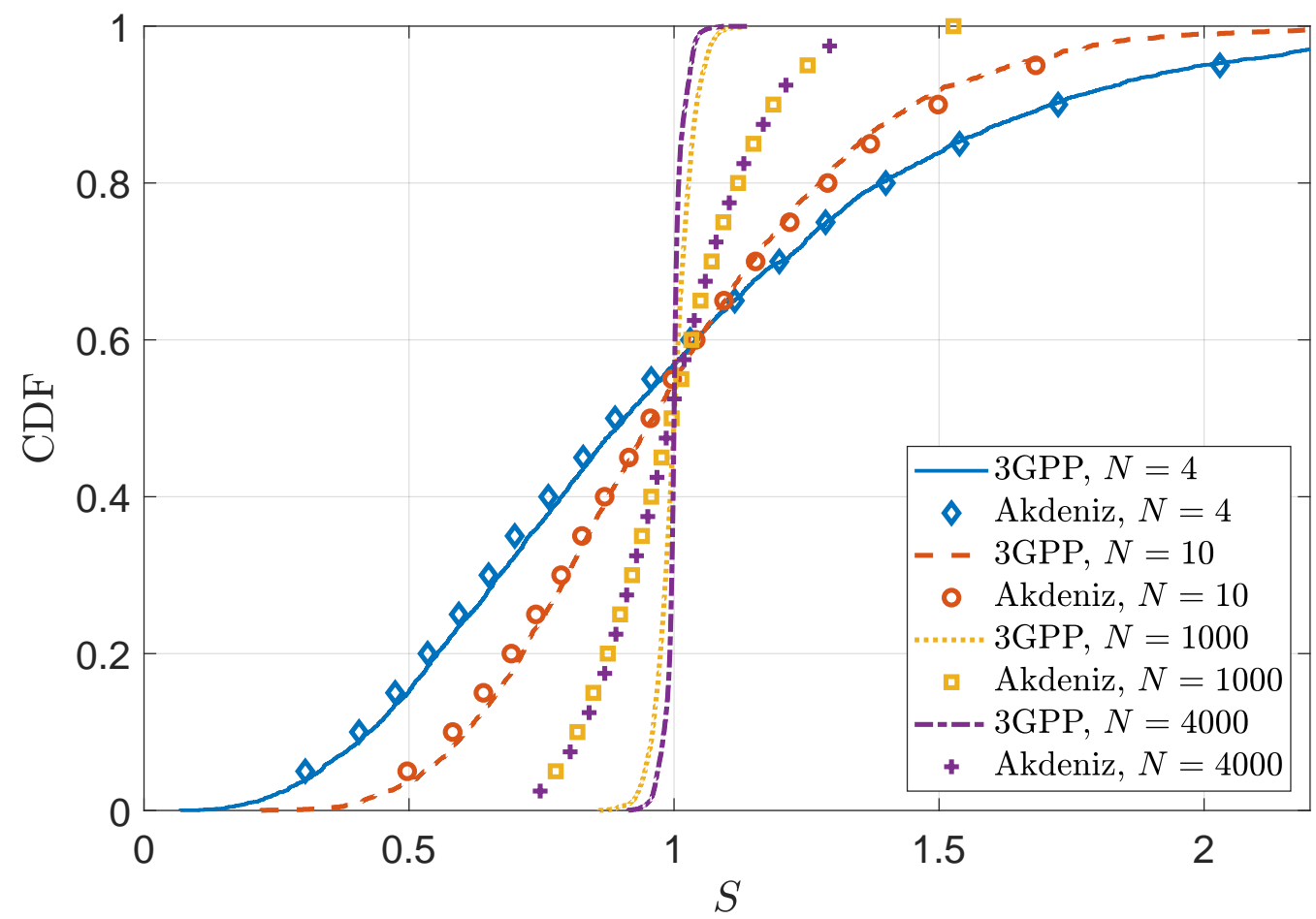

Figure 7.2: Channel hardening for two types of channel models.

give more details of the growth rate with regard to angular distributions.

In Fig. 7.4, we demonstrate the logarithmic growth rate of $\mu_{\mathrm{ULA}}$ against the number of antennas, $N$, for VM and uniform models with different parameters as shown in the legend. The analytical results in Sec. 7.3.3 were used in generating $\mu_{\mathrm{ULA}}$ for the uniform and VM distributions, respectively. Note that although the analysis in Theorem 1 predicted logarithmic growth for $\mu_{\mathrm{ULA}}$, this is hard to verify from Fig. 7.3. Hence, we substitute (7.10) into (7.9) and identify the dominant component of $\mu_{\mathrm{ULA}}$ giving $\mu_{\mathrm{ULA}} \sim m_{\text {slope }} \log (N)+C_{0}$, where $C_{0}$ is a constant and

$$
m_{\text {slope }}=\frac{2\left(f_{\phi}^{2}\left(\frac{\pi}{2}\right)+f_{\phi}^{2}\left(-\frac{\pi}{2}\right)\right)}{d}
$$

Hence, $m_{\text {slope }}$ determines how quickly $\mu_{\mathrm{ULA}}$ will grow. The uniform distribution has the highest interference growth rate, which is $m_{\text {slope }}^{\text {uniform }}=\left(\pi^{2} d\right)^{-1}$. For 


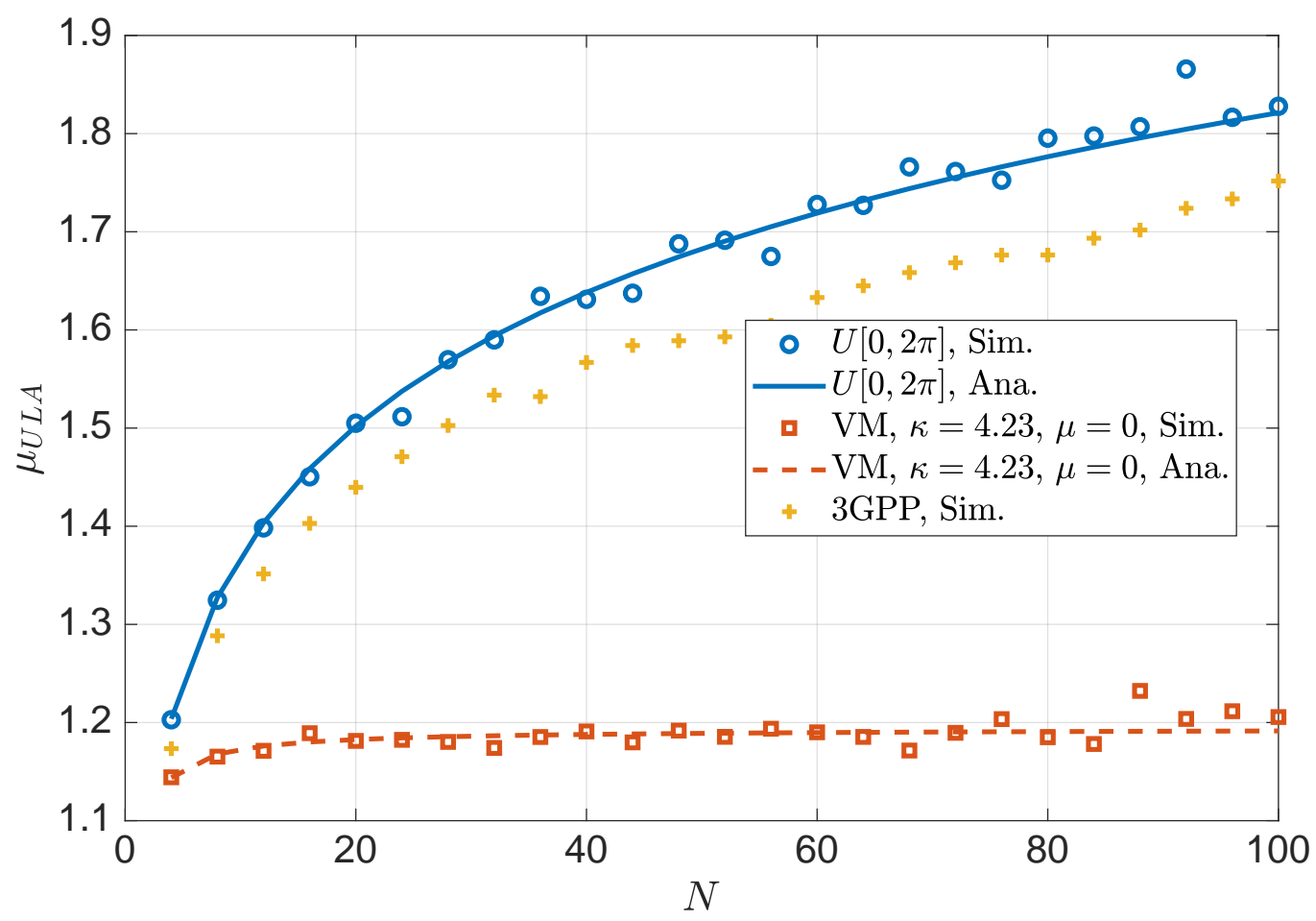

Figure 7.3: $\mu_{\mathrm{ULA}}$ vs $N$ for three different angular distributions.

the VM model, the slope depends on $\kappa$ and $\mu$. In Fig. 7.4 we observe that $\mu_{\mathrm{ULA}}$ is clearly logarithmic in $N$, as predicted, and that the slope is correctly identified by (7.10), as shown by the dotted lines which have slope $m_{\text {slope }}$.

As well as verifying the logarithmic growth, Fig. 7.4 demonstrates some interesting angular properties. For both $\kappa=4.23\left(\right.$ angle spread $\left.=30^{\circ}\right)$ and $\kappa=1.49$ (angle spread $\left.=60^{\circ}\right), \mu_{\mathrm{ULA}}$ decreases as $\mu$ is reduced from $\mu=0.52$ $\left(30^{\circ}\right)$ to $\mu=0$. This is because shifting the mean towards broadside reduces the interference inflation that occurs near end-fire. Secondly, for both $\mu=0$ and $\mu=0.52$ there is a cross-over as $N$ increases. For small $N$, increased angular spread is beneficial as it spreads the rays and reduces the chance of high interference caused by rays in close proximity. However, for high $N$ the higher angular spread puts more probability near end-fire and this begins to dominate and causes higher interference.

In Fig. 7.5, we confirm via simulation for the 3GPP parameters and via 


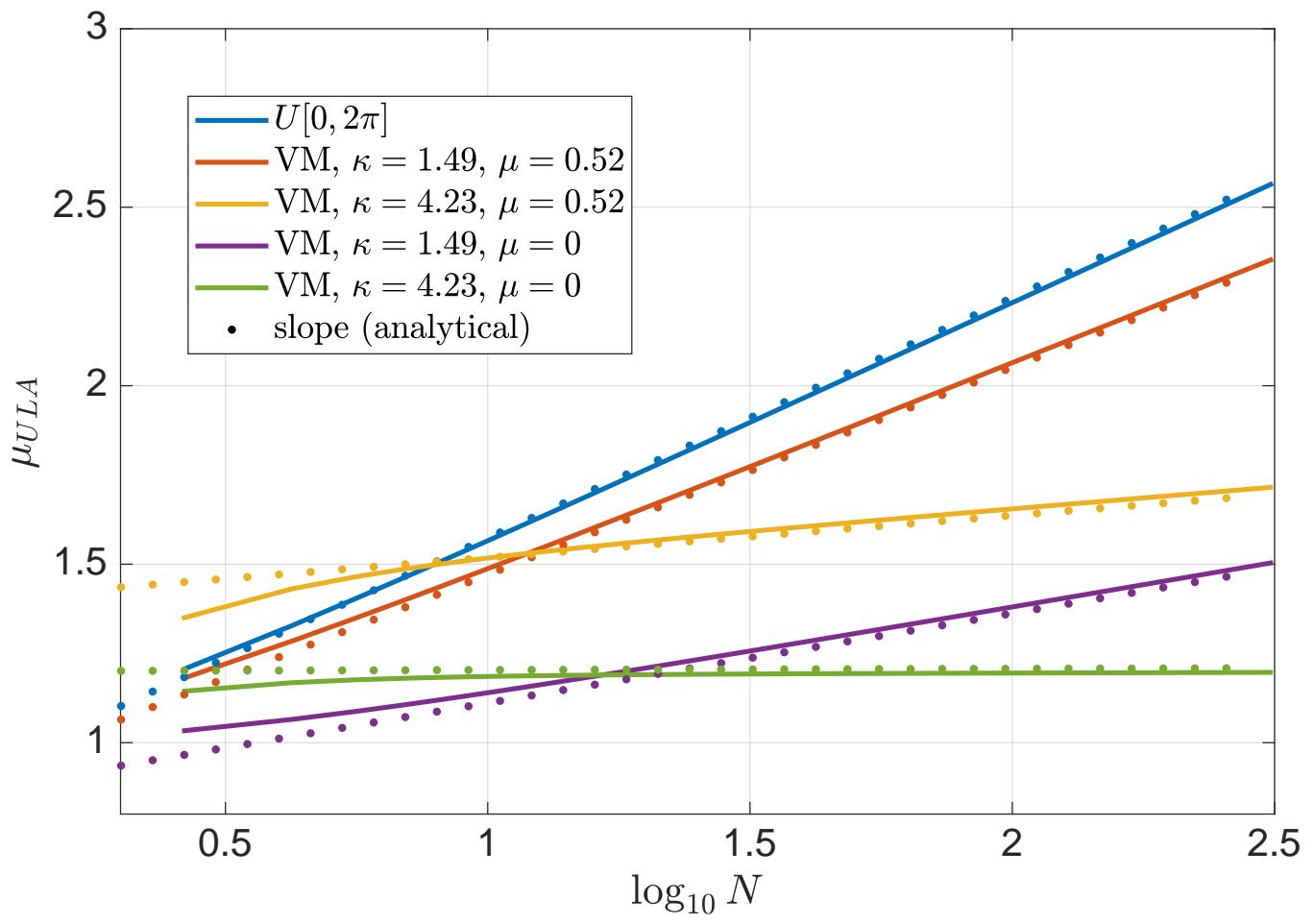

Figure 7.4: Logarithmic growth of $\mu_{\mathrm{ULA}}$.

analysis for the uniform and VM models that the mean global interference term, $\mathbb{E}\left[\eta_{i}\right]$, grows logarithmically as predicted by the analysis in Sec. 7.3.3. For the uniform case, $\phi_{i r} \sim U[0,2 \pi]$, for VM, $\kappa=4.23, \mu=0$, and for 3GPP we use the parameters considered in Fig. 7.1. For the uniform and VM models all user link gains and ray powers are equal, $\beta_{i r}=(C L)^{-1}$. For the 3GPP parameters, we also consider unequal ray powers and unequal user link gains. To avoid the substantial extra variation caused by shadowing models with large arrays we employ a simple deterministic model for these powers. The link gains decay exponentially from user 1 to user $K$ such that $\beta_{K}=\frac{1}{10} \beta_{1}$ and the cluster powers behave similarly. The desired user is then randomly allocated one of the $K$ distinct link gains. The levels are then adjusted to give the same total power as in the uniform and VM models, and subrays in a particular cluster all have the same power as assumed in [154]. Fig. 7.5 shows the same logarithmic growth as Fig. 7.3, confirming the analysis. 


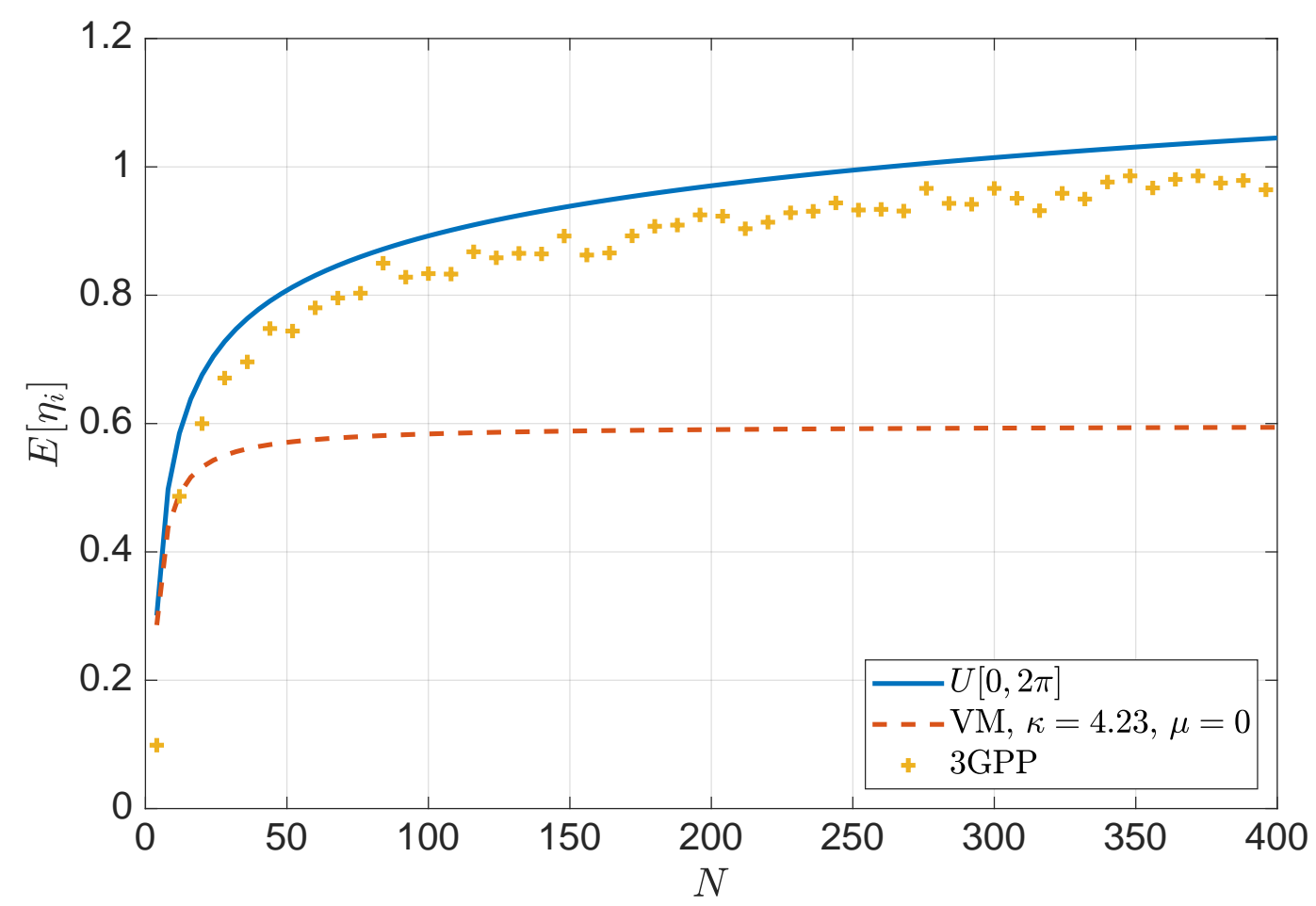

Figure 7.5: $\mathbb{E}\left[\eta_{i}\right]$ vs $N$ for three angular distributions.

In Fig. 7.6, we show the variation of $\mathbb{E}\left[\eta_{i}\right]$ with $N$ for the 3GPP channel model. The parameters are identical to Fig. 7.5 except the channel powers, $\beta_{1}, \beta_{2}, \cdots, \beta_{K}$, are chosen using the classic path-loss and shadowing model given in Sec. 4.4. The parameters used for the path loss and shadowing are: path loss exponent $\gamma=3.5$ and the standard deviation of the shadowing is $\zeta=6 \mathrm{~dB}$. Note that the Fig. 7.5 simulations for the 3GPP model already show simulation error. When the extra variation due to shadowing and path loss is added in, the simulations become extremely variable. In Fig. 7.6, 4000 simulations are run (as in Fig. 7.5) but this is far too small to see accurate results. In practice, extremely long simulation runs are required in this scenario.

In Fig. 7.7, we evaluate the channel hardening and FP results for a UPA with $K=2$ and an increasing number of antennas. We adopt the angular models from [36]. The azimuth central angle follows a wrapped Gaussian distribution $\left(\phi_{c} \sim \mathcal{N}\left(0^{\circ}, \sigma_{c}^{2}\right)\right)$ with a Laplacian offset distribution $\left(\Delta_{c, s} \sim\right.$ 


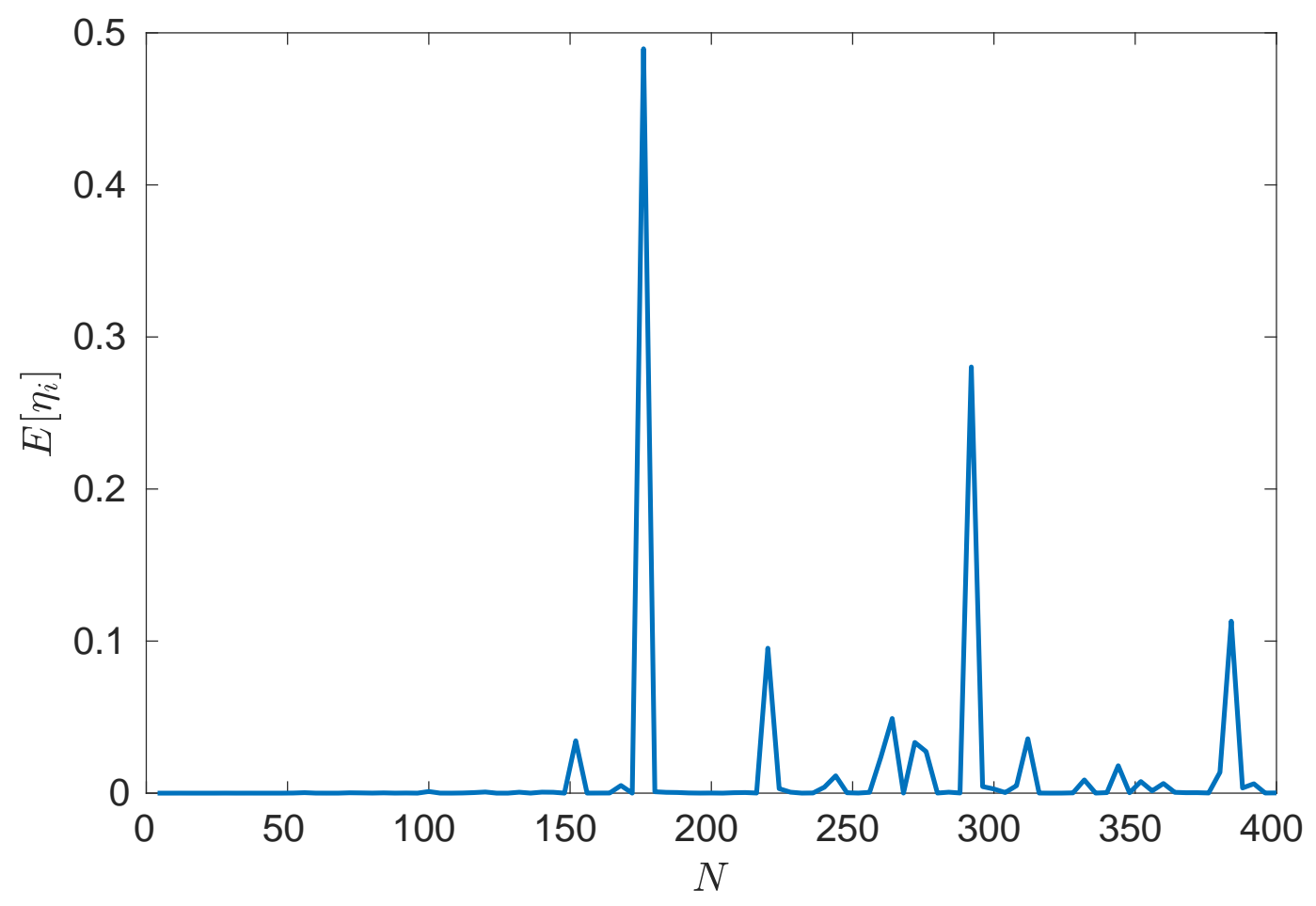

Figure 7.6: $\mathbb{E}\left[\eta_{i}\right]$ vs $N$ for the 3GPP model including the effects of shadowing for the users.

$\left.\mathcal{L}\left(1 / \sigma_{s}\right)\right)$. Both the central and offset angles for elevation have Laplacian distributions with $\theta_{c} \sim \mathcal{L}\left(1 / \hat{\sigma}_{c}\right)$ (centred on $90^{\circ}$ ) and $\delta_{c, s} \sim \mathcal{L}\left(1 / \hat{\sigma}_{s}\right)$, respectively. We adopt the following parameters: the number of clusters is $C=20$, the number of subrays within a cluster is $L=20, \sigma_{c}=31.64^{\circ}$ and $\sigma_{s}=24.25^{\circ}$ from [154] and we use the upper $90 \%$ of the lognormally distributed values of $\hat{\sigma}_{c}$ and $\hat{\sigma}_{s}$ from [36] in order to give a wide angular spread, which is $6.12^{\circ}$ and $1.84^{\circ}$, respectively (Scenario Wide). As in Fig. 7.1 and Fig. 7.2, equal powers for subrays are assumed and $\beta_{i r}=1 /(C L)$ for simplicity. From Fig. 7.7, we see that, similar to ULA, FP and channel hardening also occur for the UPA structure. However, the gap between the 3GPP and Rayleigh channel in Fig. 7.7 is wider than for a ULA. Hence, the smaller azimuth footprint of the UPA slows down FP.

In Fig. 7.8, we show both the simulated and analytical results for $\mu_{\text {UPA }}$ 

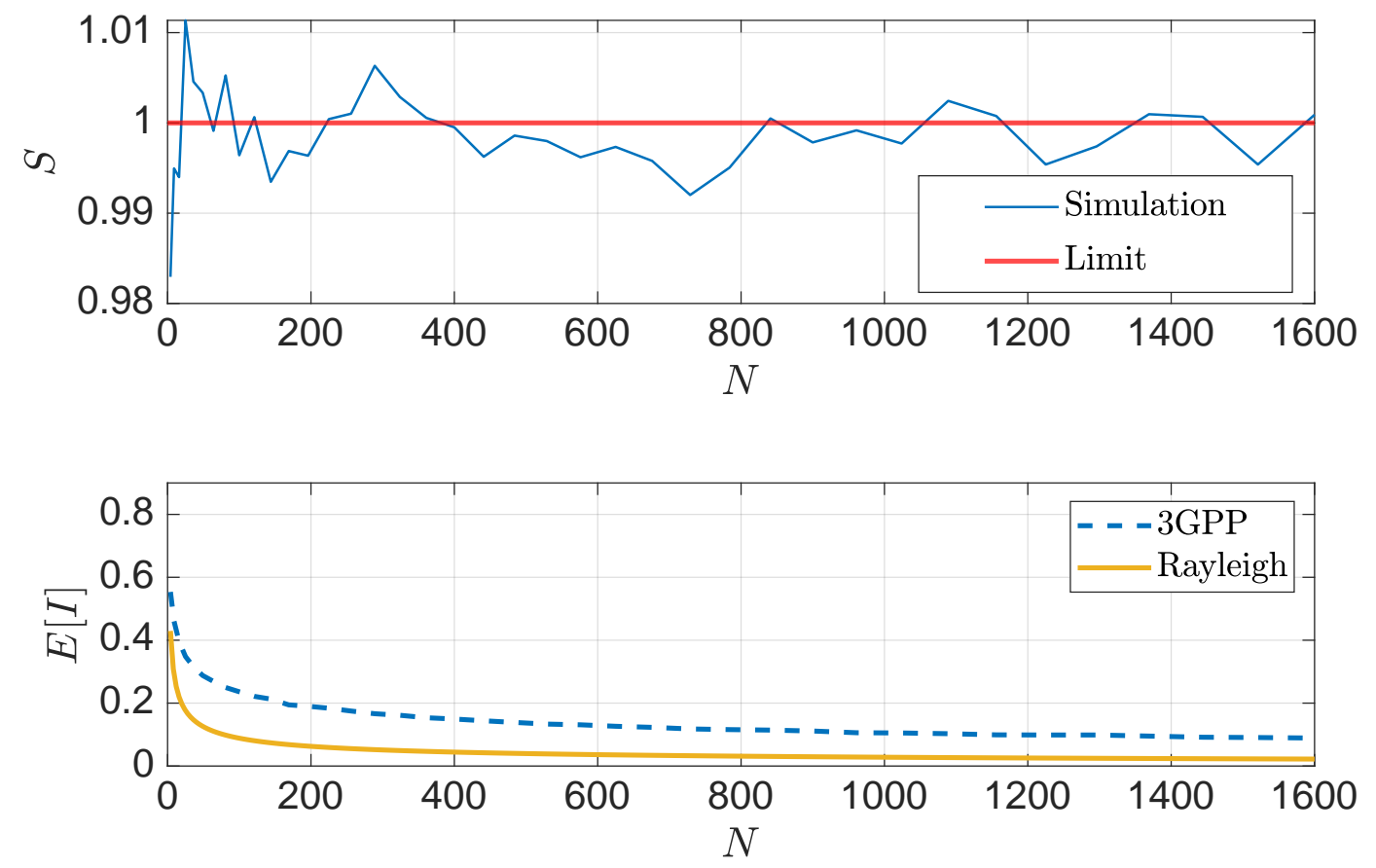

Figure 7.7: Channel hardening and FP for UPA.

with a uniform angular distribution for both azimuth $(U[0,2 \pi])$ and elevation angles $(U[0, \pi])$. The analytical results are from Secs. 7.4.3. We can see that the analysis agrees well with the simulation. We also show the simulated values of $\mu_{\mathrm{UPA}}$ for two scenarios. Scenario Wide uses the angular parameters in Fig. 7.7. Scenario Narrow uses $C=3, L=16, \sigma_{c}=14.4^{\circ}$ and $\sigma_{s}=6.24^{\circ}$ and the lower $10 \%$ of the lognormally distributed values of $\hat{\sigma}_{c}$ and $\hat{\sigma}_{s}$ from [36]. As with the ULA, the growth rates of $\mu_{\mathrm{UPA}}$ are different for all three models, due to the differences in both the azimuth and elevation angular distributions.

In Fig. 7.9, the logarithmic growth of $\mu_{\mathrm{UPA}}$ with different angular spreads in both azimuth and elevation angles is presented. As we can see, the narrower the angular spread is, the quicker $\mu_{\mathrm{UPA}}$ grows. Note that the lower curves show reasonably clearly that growth is linear in $\log (N)$, logarithmic in N. Even here, the simulation errors obscure the linearity a little. For the narrow scenario, up to the number of antennas shown, the curve is still in its 


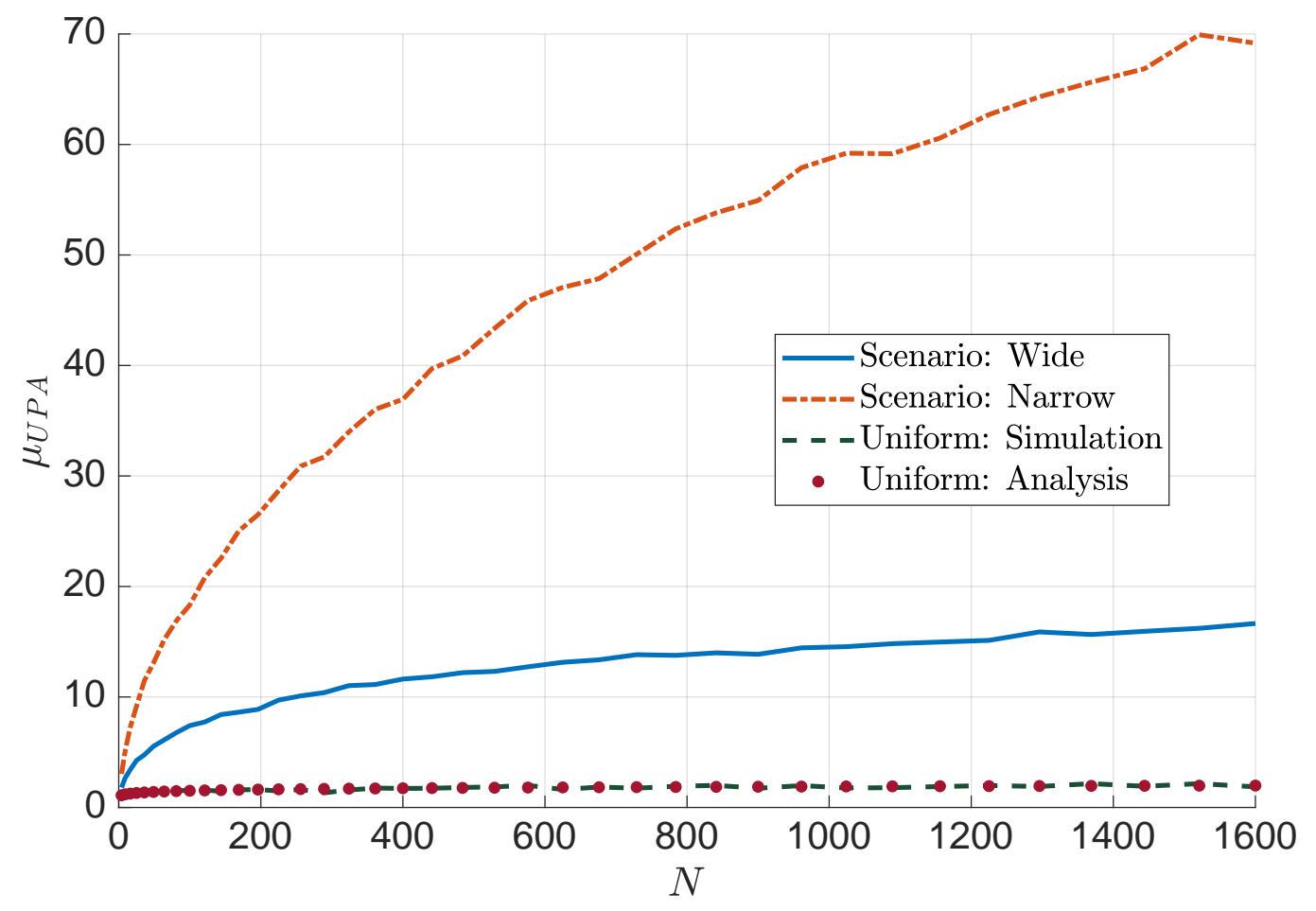

Figure 7.8: $\mu_{\mathrm{UPA}}$ vs $N$ for wide and narrow angular distributions for UPA.

initial stage before the linearity (the high $\mathrm{N}$ regime) begins. Given that the graph already goes up to over 1000 antennas $\left(\log _{10}(N)>3\right)$, the $x$-axis was stopped here as it is already a large number of antennas and simulations for large arrays take a very long time due to the large variability of interference.

In Fig. 7.10, we confirm via simulation for the 3GPP parameters (adopting Scenario Wide and Scenario Narrow parameters in Fig. 7.8) that the mean global interference term, $\mathbb{E}\left[\eta_{i}\right]$, grows logarithmically as predicted by the analysis in Sec. 7.4.3 ${ }^{8}$. We consider unequal ray powers and unequal user link gains as in Fig. 7.5. The results in Fig. 7.10 are also shown on a logarithmic scale in Fig. 7.11. Again, note that the wide scenario shows clearly that growth is linear in $\log (N)$. For the narrow scenario, the curves is still in its initial stage before the linearity begins.

In Fig. 7.12, we show the instantaneous behavior of $\eta_{i}$ that causes $\mathbb{E}\left[\eta_{i}\right]$ 


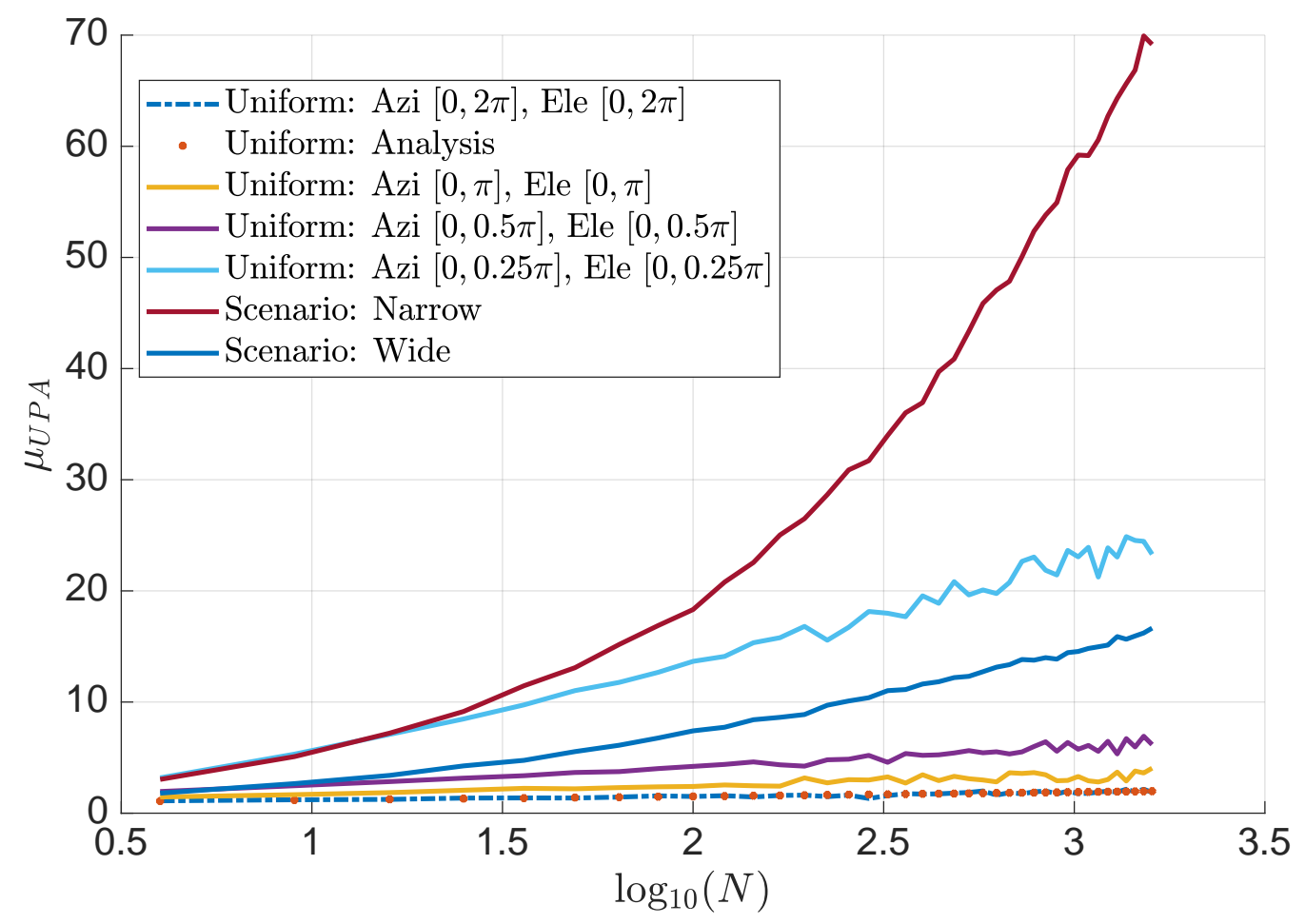

Figure 7.9: Logarithmic growth of $\mu_{\mathrm{UPA}}$ for UPA.

to grow. A simple channel is assumed with 20 paths, half-wavelength antenna spacing in a ULA, unit power rays, $\alpha=10$ and all rays have a $U[0,2 \pi]$ distribution. With no control over the users entering the system, occasional large inteference values occur as interfering user rays nearly align with the desired user. This is demonstrated by the spikes in the curve labelled as "no scheduling." Note that these spikes keep occurring even for massive antenna numbers, up to 2000. In contrast, we also show that the trivial scheduling scheme introduced in Sec. 7.3.4 with a protection target of $\epsilon=0.1$, equivalent to an angular separation of $0.57^{\circ}$. Here, the value of $\eta_{i}$ is well-behaved and decays to zero. 


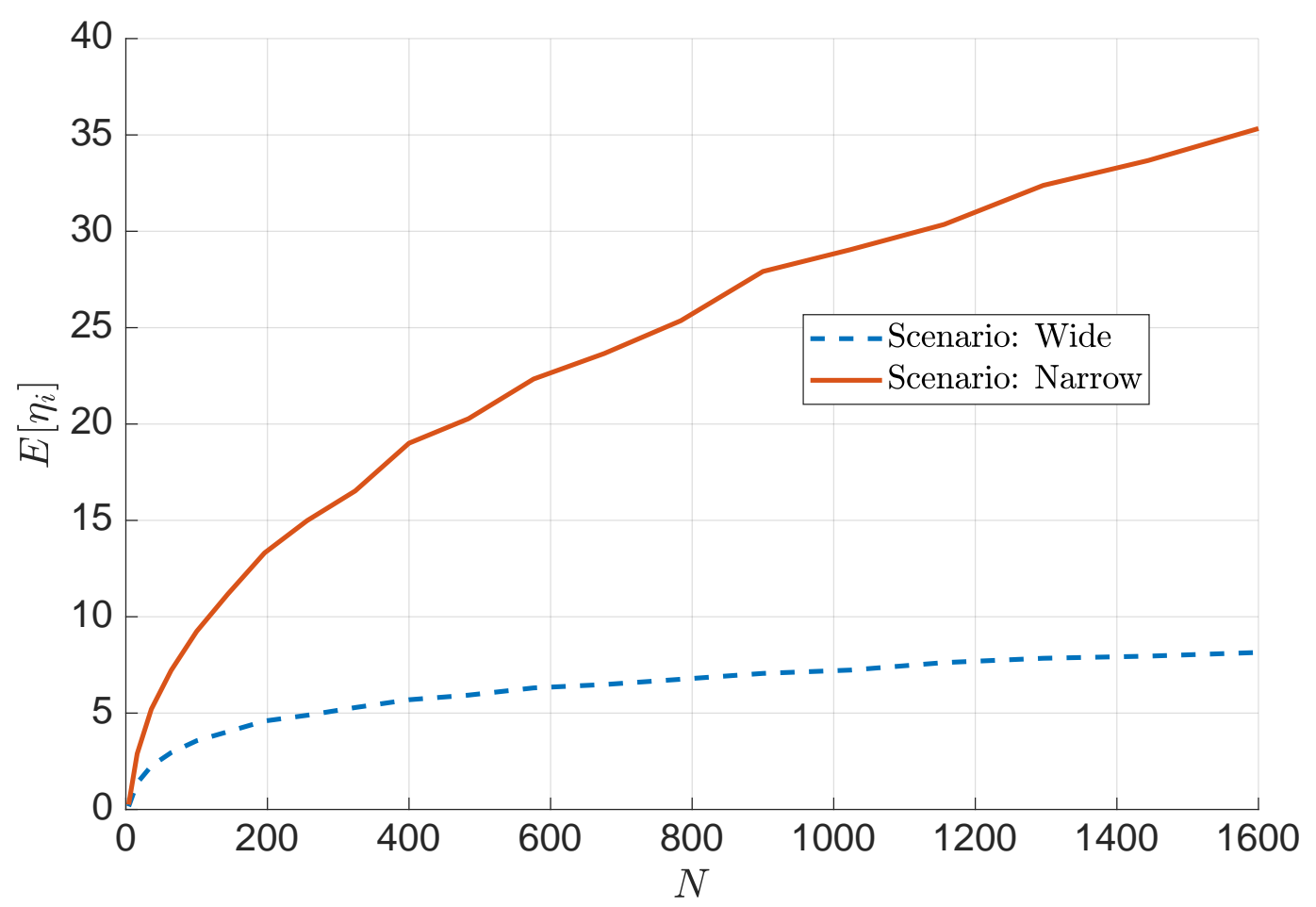

Figure 7.10: $\mathbb{E}\left[\eta_{i}\right]$ vs $N$ for two angular distributions.

\subsection{Conclusion}

The fundamental properties of massive MIMO have been identified with great generality for a broad class of ray-based models with a ULA or a UPA at the BS. The generality and insight possible is considerably greater than can be achieved with statistical channel models. In particular, we show that channel hardening may or may not occur depending on the model used and FP is guaranteed for all continuous angular distributions. Although LSP will not normally hold, as the mean interference grows logarithmically relative to the desired channel, the implications for massive MIMO are excellent. As the number of users grows, the mean interference does grow relative to the desired channel but extremely slowly and this is further reduced by practical considerations, such as the attenuation of end-fire radiation caused by typical array patterns. In addition, we prove that this mean interference growth can 


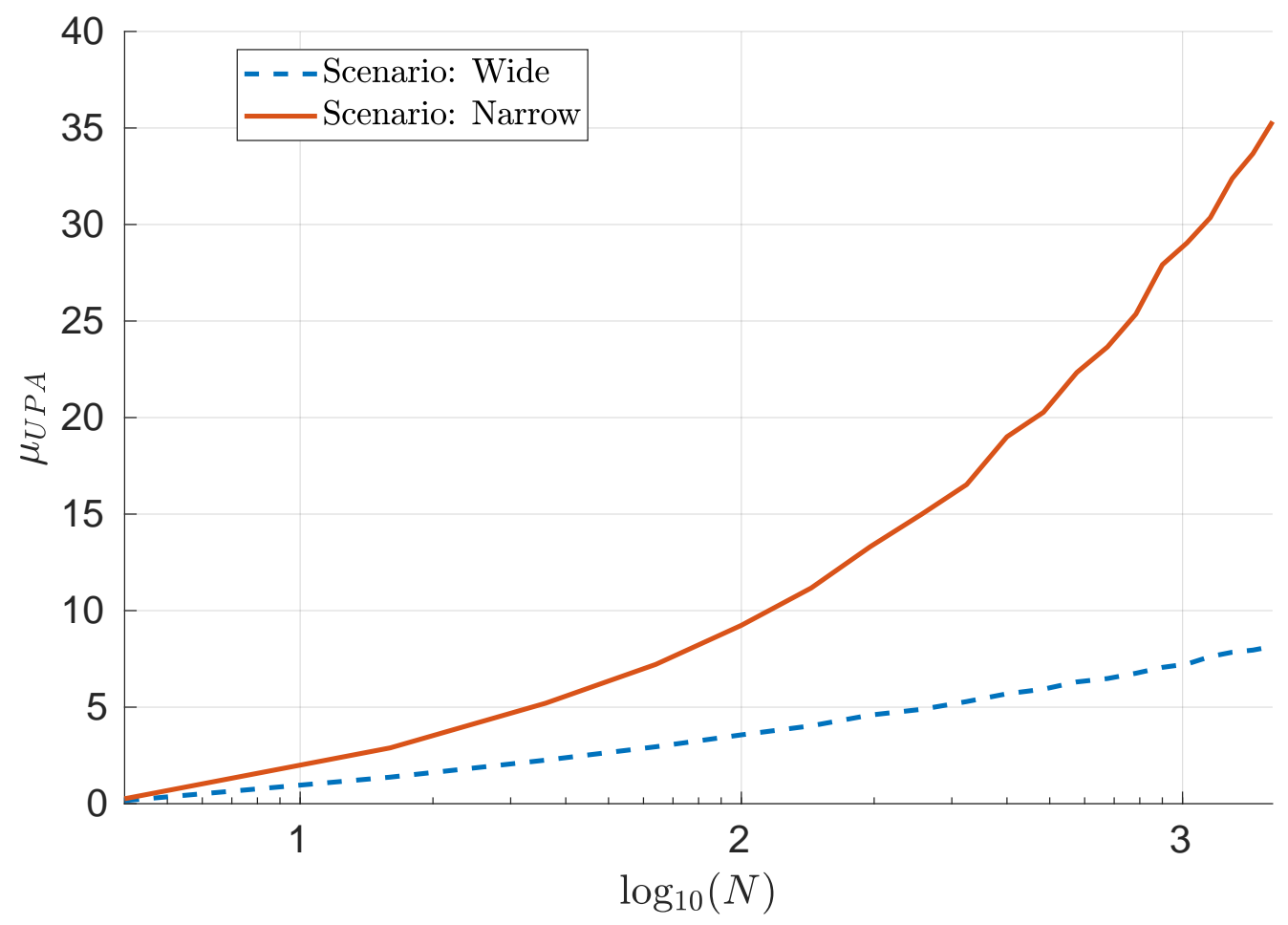

Figure 7.11: $\mathbb{E}\left[\eta_{i}\right]$ vs $\log _{10} N$ for two angular distributions.

be avoided by trivial scheduling schemes. 


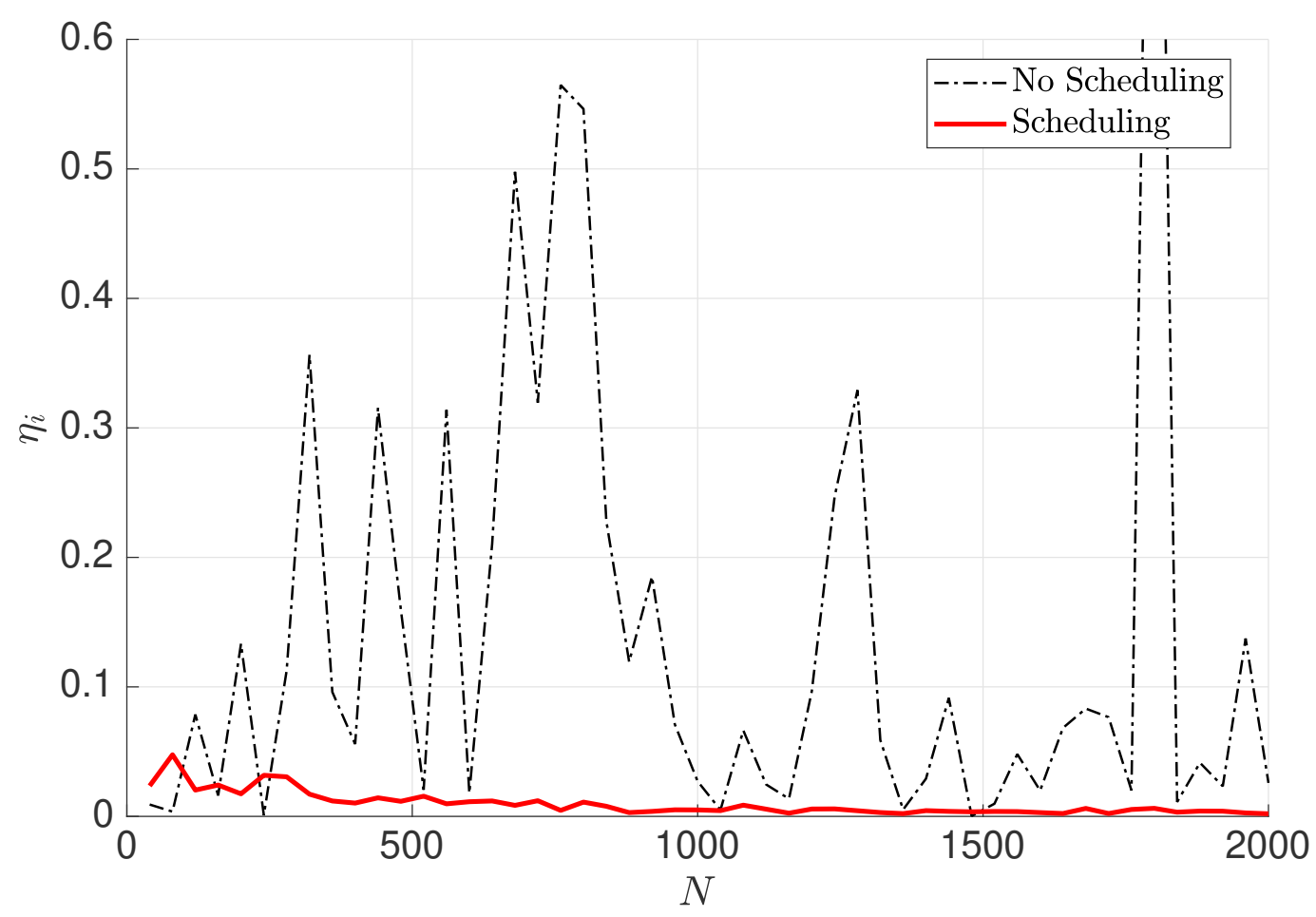

Figure 7.12: $\eta_{i}$ vs $N$ with and without scheduling. 


\section{Conclusions and Future Work}

This chapter summarizes the research work presented in this thesis, and then provides several future research directions.

\subsection{Conclusions}

This thesis analyses the system performance of MU-MIMO wireless communication systems using a variety of linear processing schemes based on analog MRC and digital MRC. The analysis caters for a variety of channels including i.i.d Rayleigh and correlated Rayleigh. Numerical results are also given for Ricean channels. In addition, the fundamental properties of FP and channel hardening are considered, both in terms of asymptotic massive MIMO behaviour as well as MRC behaviour, where we employ a very general class of ray-based models. These topics are important for understanding next generation wireless communication systems as future systems are likely to operate with large antenna arrays employing low complexity processing techniques. A number of analytical, simulated results, and useful insights are presented, leading to an improved understanding for future system implementation. Specific conclusions are given in the following. 


\subsubsection{Distributed Antenna Systems}

Chapter 4 and Chapter 6 analyse the system performance for various distributed BS layouts. Chapter 4 compares the system performance of digital and analog MRC/MF in both centralized and distributed systems. We show that digital MRC benefits from distributed clusters and analog MRC has a relatively good performance in a centralized system. The impact of Rice K factors is analysed, showing that the gap between digital MRC and analog MRC shrinks with increasing Rice $\mathrm{K}$ factor in a centralized system but remains in distributed systems. Chapter 6 presents analytical expressions for the expected per user SINR in an uplink distributed MIMO system with twostage beamforming techniques. Two- and four-cluster BS layouts are considered with four beamforming strategies (A-D, D-D, A-ZF and D-ZF). The first stage considers either analog or digital MRC, followed by the second stage of either digital MRC or digital ZF. We also present the asymptotic behaviour of A-D and D-D strategies. We show a $\pi / 4$ performance ratio in a centralized configuration, while for the distributed layout, the relative performance of A-D and D-D varies depending on the distribution of interference among the base stations. Finally, we show that the performance gap between A-D and DD increases with the number of BS clusters, which agrees with our analytical results in Chapter 4.

- Analog MRC has the benefit of low power consumption, low complexity, and hardware costs. It is an ideal candidate for massive MIMO systems if the performance gap between analog MRC and digital MRC is reasonable. In our work, we show that if only considering local processing for distributed clusters, the performance of analog MRC degrades compared with digital MRC when the number of clusters increases. Thus, analog MRC is more suitable in a centralized system 
when a strong Rice $\mathrm{K}$ factor is anticipated, in other words, in a more open environment.

- Two-stage processing makes the use of analog MRC at local processing even more promising. A-D only requires phase shifters in the local processing and one RF chain per cluster for central processing. Thus, A-D further reduces the complexity and hardware requirements compared with D-D and D-ZF for distributed systems. Furthermore, our results show that A-D can outperform D-D at lower SNR and even at moderate SNR levels the performance gap is small.

\subsubsection{Spatial Correlation for Distributed and Centralized Sys- tems}

Chapter 4 and Chapter 5 consider the impact of correlation on MU-MIMO systems. Chapter 4 presents novel analytical expressions of expected per-user SINR for both centralized and distributed systems with arbitrary correlation models. Although distributed systems reduce the adverse impact of correlation by dividing the antennas into multiple clusters, the correlation within the cluster still exists. Thus, we extend our work in Chapter 4 to the analysis of various correlation models in a centralized system in Chapter 5. The analysis demonstrates that SIR decreases when the user correlation matrices are identical, but increases when they are different. Thus, the system performance is critically dependent on the correlation scenario. We have shown that for uncorrelated fading the SINR converges to a constant. For fully correlated channels SINR converges to zero for equal correlation matrices, whereas for unequal correlation matrices SINR converges to zero or a constant, depending on the correlation model.

- Novel insights are given that correlation can improve system perfor- 
mance and users' angular distribution is the key. Large amplitude correlation helps the signal relative to interference, thus enhancing SINR, unless the phases are very similar when the SINR is adversely affected by large interference. Hence, as long as there is diversity in the phase parameters, increasing correlation is beneficial to performance.

- In a centralized system, our analysis shows that analog MRC and digital MRC have the same asymptotic behaviour under the assumption of perfect correlation. This implies analog MRC will work well whereas digital MRC degrades when high correlation presents in the system. This further explains why a strong Rice $\mathrm{K}$ factor would help analog MRC.

- The interference behaviour under perfect correlation with two special correlation models, exponential and one-ring are studied. We find that the asymptotic behaviour of interference mainly depends on the angular distribution of users.

\subsubsection{Asymptotic Analysis for Massive MIMO}

There are two cases to distinguish when referring to asymptotic analysis for massive MIMO, single-sided massive MIMO and double-sided massive MIMO. If there is only a finite number of users to be served by increasing the number of antenna elements, we refer to this as single-sided massive MIMO. When growing the number of antennas, ideally, more users are likely to be served, leading to a system that becomes large both in users and antennas. This is referred as double-sided massive MIMO.

The effects of correlation on the asymptotic behaviour of double-sided massive MIMO for analog and digital MRC is analysed in Chapter 5. The findings suggest that the system performance is improved where there is perfect correlation within one user's channel but a random phase distribution 
among users. Thus, in some cases, correlation is beneficial and can help system performance. From the derived expression of SINR for i.i.d Rayleigh fading, around 21.5\% performance loss occurs with analog MRC compared with digital MRC. The system performance analysis under perfect correlation suggests that digital and analog MRC would have the same asymptotic behaviour.

Chapter 6 concerns the asymptotic behaviour of expected SINR for the two-stage processing techniques D-D and A-D in a single-sided massive MIMO system. The analysis shows that A-D has a performance loss of approximately $21.5 \%$ compared to D-D in centralized systems. However, D-D performs significantly better when interference is scattered among clusters while A-D actually outperforms D-D when the dominant interferers coincide at one BS cluster.

Two key principles behind the success of massive MIMO are FP (singlesided massive MIMO) and channel hardening. With FP, the use of large numbers of antennas offers an implicit interference reduction mechanism, and enables the use of low complexity signal processing algorithms. The bulk of the theoretical work on FP and channel hardening has utilized classical statistical channel models. In Chapter 7, we analyse FP and channel hardening for ray-based channel models and, furthermore, we propose a novel system performance metric, LSP (for double-sided massive MIMO). The fundamental properties of massive MIMO have been identified with great generality for a broad class of ray-based models with a ULA or a UPA at the BS. The generality and insight possible is considerably greater than can be achieved with statistical channel models. In particular, we show that channel hardening may or may not occur depending on the model used and FP is guaranteed for all continuous angular distributions. Although LSP will not normally hold, as the mean interference grows logarithmically relative to the desired 
channel, the implications for massive MIMO are excellent. In a double-sided massive MIMO system, the mean interference does grow relative to the desired channel but extremely slowly and this is further reduced by practical considerations, such as the attenuation of end-fire radiation caused by typical array patterns. In addition, we prove that this mean interference growth can be avoided by trivial scheduling schemes.

- For ray-based models, we show that channel hardening may or may not occur depending on the nature of the model and FP is guaranteed for all models where the ray angles are continuous random variables (as assumed by all models to date).

- For LSP, we derive remarkably simple expressions which relate the asymptotic interference behavior to system size, antenna spacing the ray distribution. We demonstrate that LSP will not normally hold as the mean interference power grows logarithmically relative to the mean power of the desired user channel as the system size increases. Although the interference eventually dominates the desired channel, the growth is very slow and is further attenuated by practical factors such as the likely propagation environment and the typical array patterns employed. In addition, we prove that trivial scheduling schemes can retain LSP, and thus increase the robustness of massive MIMO performance.

\subsection{Future Work}

In this section, we propose the following research areas for further exploration: 


\subsubsection{Multi-user Scheduling for Massive MIMO}

In Chapter 7, it was shown that simple scheduling can force LSP to hold. Hence, it is expected that scheduling could have a large impact on ray-based channels. However, choosing the optimal scheduling method is a polynomial time hard problem, and has a computational complexity of $O\left(\begin{array}{c}K \\ N_{\text {user }}\end{array}\right)$, where $N_{\text {user }}$ is the number of potential users and $K$ is the number to be selected. For large systems, this complexity quickly becomes infeasible. Also, considering the computational time for measuring the system performance based on the chosen users, the computational burden is even heavier. Thus, the design of efficient scheduling methods for ray-based channels is in high demand.

\subsubsection{Multi-cell Interference Analysis for Two-stage Hybrid MRC}

In Chapter 6, we only consider the system performance of two-stage hybrid MRC, with A-D and D-D in a single cell. Since MRC does not have the ability to mitigate interference, it would be useful to analyse the system performance when taking the adjacent cells' interference into consideration. The same research interest also applies in Chapter 5. 


\section{Appendix}

The work in these appendices is taken from [149].

\subsection{Proof of Theorem 1}

We note that

$$
\mathbb{E}\left[e^{-j s \hat{\phi}_{i r}}\right]=\int_{-2 \pi d}^{2 \pi d} e^{-j s x} f_{\hat{\phi}}(x) d x
$$

where $f_{\hat{\phi}}(\cdot)$ is PDF of $\hat{\phi}_{i r}$. Now, $\hat{\phi}_{i r}=2 \pi d \sin \phi_{i r}$ is a non one-to-one transformation of $\phi_{i r}$. Using standard transformation theory, we obtain

$$
f_{\hat{\phi}}(x)=\frac{p(x)}{\sqrt{l^{2}-x^{2}}},-l \leq x \leq l
$$

where $l=2 \pi d$ and

$$
p(x)=\left\{\begin{array}{l}
f_{\phi}\left(\sin ^{-1}\left(\frac{x}{l}\right)\right)+f_{\phi}\left(\pi-\sin ^{-1}\left(\frac{x}{l}\right)\right), x \geq 0 \\
f_{\phi}\left(\sin ^{-1}\left(\frac{x}{l}\right)\right)+f_{\phi}\left(-\pi-\sin ^{-1}\left(\frac{x}{l}\right)\right), x<0 .
\end{array}\right.
$$


Hence, (9.1) is rewritten as

$$
\mathbb{E}\left[e^{-j s \hat{\phi}_{i r}}\right]=\int_{-l}^{l} e^{-j s x} \frac{p(x)}{\sqrt{l^{2}-x^{2}}} d x
$$

Using the notation in [155, Eq.1, p. 15], the Fourier transform (FT) of a function $f(x)$ can be written as

$$
g(y)=\int_{-\infty}^{\infty} f(x) e^{-j 2 \pi x y} d x
$$

If we set $y=\frac{s}{2 \pi}$, then

$$
g\left(\frac{s}{2 \pi}\right)=\int_{-\infty}^{\infty} f(x) e^{-j s x} d x
$$

Using the Heaviside function, $H(x)$, we can write (9.4) as a FT in the same format as (9.6) as follows,

$$
\mathbb{E}\left[e^{-j s \hat{\phi}_{i r}}\right]=\int_{-\infty}^{\infty} e^{-j s x}(H(x+l)-H(x-l)) \frac{p(x)}{\sqrt{l^{2}-x^{2}}} d x .
$$

Hence, defining $f(x)=(H(x+l)-H(x-l)) \frac{p(x)}{\sqrt{l^{2}-x^{2}}}$, allows $\mathbb{E}\left[e^{-j s \hat{\phi}_{i r}}\right]$ to be computed as the FT of $f(x)$.

This formulation is particularly useful as we can now leverage known results on the asymptotics of FTs as $s \rightarrow \infty$ [155]. These results depend on the singularities of $f(x)$ so we first discuss the nature of these singularities. Clearly, $f(x)$ has singularities at $x= \pm l$ and at any singularities of $p(x)$. Note that the singularities at $x= \pm l$ are infinite discontinuities (indicating that the value of $f(x)$ will grow infinitely large as $x$ approaches $\pm l$ ). In contrast, the singularities of $p(x)$ are never infinite discontinuities for any proposed, practical angular distribution models. Models such as the wrapped Gaussian have no singularities inside $(-l, l)$ while the Laplacian has only a non-differentiable 
point at the peak. Hence, the singularities at $x= \pm l$ are the worst. The general principle presented in [155, p. 55] is that the 'worst' singularity ${ }^{1}$ of a function contributes the leading term to the asymptotic expression for its FT. Thus in our case, we only need to consider the two singularities at $x= \pm l$. Near $x_{1}=-l, f(x)$ behaves like $F_{1}(x)=H(x+l) p(-l)(2 l(l+x))^{-1 / 2}$ and similarly near $x_{2}=l, f(x)$ behaves like $F_{2}(x)=(1-H(x-l)) p(l)(2 l(l-x))^{-1 / 2}$. Rewriting, we obtain

$$
\begin{gathered}
F_{1}(x)=\frac{H(x+l) p(-l)}{\sqrt{2 l}}|x+l|^{-\frac{1}{2}}, \\
F_{2}(x)=\frac{p(l)}{\sqrt{2 l}}|x-l|^{-\frac{1}{2}}-\frac{H(x-l) p(l)}{\sqrt{2 l}}|x-l|^{-\frac{1}{2}} .
\end{gathered}
$$

From [155, Theorem 19, p. 52], we know that if a generalised function, $f(x)$, has a finite number of singularities at $\left\{x=x_{1}, x_{2}, x_{3}, \ldots, x_{m}\right\}$, and for each of them $f(x)-F_{m}(x)$ has absolutely integrable $N^{\text {th }}$ order derivatives in an interval including $x_{m}$, where $F_{m}(x)$ is a linear combination of functions of type $\left|x-x_{m}\right|^{\beta},\left|x-x_{m}\right|^{\beta} \operatorname{sgn}\left(x-x_{m}\right),\left|x-x_{m}\right|{ }^{\beta} \log \left|x-x_{m}\right|,\left|x-x_{m}\right|^{\beta} \log \mid x-$ $x_{m} \mid \operatorname{sgn}\left(x-x_{m}\right)$, and if $f^{(N)}(x)$ is well behaved at infinity, then $g(y)$, the FT of $f(x)$, satisfies $g(y)=\sum_{m=1}^{M} G_{m}(y)+o\left(|y|^{-N}\right)$, as $|y| \longrightarrow \infty$, where $G_{m}(y)$ is the FT of $F_{m}(x)$. Using this, we have

$$
g\left(\frac{s}{2 \pi}\right) \sim G_{1}\left(\frac{s}{2 \pi}\right)+G_{2}\left(\frac{s}{2 \pi}\right),
$$

where $G_{1}$ and $G_{2}$ are the FTs of $F_{1}(x)$ and $F_{2}(x)$ in (9.8) and (9.9) and $\sim$ denotes asymptotic equivalence defined in [132, p. 15]. From [155, Table 1, p. 43], the

\footnotetext{
${ }^{1}$ The singularity $x=x_{m}$ of a function, $f(x)$, is worst if $f(x)$ is of order $\left|x-x_{m}\right|^{\beta}$ near $x_{m}$ and $\beta$ is the smallest value for all singularities [155, p. 55].
} 
FTs required are

$$
\begin{aligned}
\mathcal{F}\left(|x-l|^{-\frac{1}{2}}\right) & =e^{-2 \pi j l y}|y|^{-\frac{1}{2}}, \\
\mathcal{F}\left(H(x+l)|x+l|^{-\frac{1}{2}}\right) & =e^{2 \pi j l y-\frac{1}{4} j \pi \operatorname{sgn}(y)}|2 y|^{-\frac{1}{2}}, \\
\mathcal{F}\left(H(x-l)|x-l|^{-\frac{1}{2}}\right) & =e^{-2 \pi j l y-\frac{1}{4} j \pi \operatorname{sgn}(y)}|2 y|^{-\frac{1}{2}} .
\end{aligned}
$$

Using (9.11), we obtain

$$
\begin{aligned}
g\left(\frac{s}{2 \pi}\right) & \sim G_{1}\left(\frac{s}{2 \pi}\right)+G_{2}\left(\frac{s}{2 \pi}\right) \\
& =\sqrt{\frac{\pi}{l s}}\left(\frac{p(-l)}{\sqrt{2}} e^{j\left(l s-\frac{\pi}{4}\right)}+p(l) e^{-j l s}-\frac{p(l)}{\sqrt{2}} \sqrt{\frac{\pi}{s}} e^{-j\left(l s+\frac{\pi}{4}\right)}\right) .
\end{aligned}
$$

Substituting $p(l)=2 f_{\phi}\left(\frac{\pi}{2}\right), p(-l)=2 f_{\phi}\left(\frac{-\pi}{2}\right)$ and $l=2 \pi d$ into (9.12), and after some simplification we obtain the result in Theorem 1.

\subsection{Proof of Theorem 2}

We drop the subscripts to re-express $M_{v, w}$ as follows,

$$
M=\mathbb{E}_{\theta}\left[\mathbb{E}_{\phi}\left[e^{-j 2 \pi \nu \sin \theta \sin \tilde{\phi}}\right]\right]
$$

where $\tilde{\phi}=\phi-\Delta+\frac{\pi}{2}$. We get

$$
M=\int_{-\frac{\pi}{2}}^{0}\left\{\mathbb{E}_{\phi}\left[e^{-j 2 \pi \nu|\sin \theta| \sin \tilde{\phi}}\right]\right\} f(\theta) d \theta+\int_{0}^{\frac{\pi}{2}}\left\{\mathbb{E}_{\phi}\left[e^{-j 2 \pi \nu|\sin (\theta)| \sin \tilde{\phi}}\right]\right\} f(\theta) d \theta
$$

where $\tilde{\tilde{\phi}}=-\tilde{\phi}$. Setting $s=\nu, d=|\sin \theta|$, allows us to use Theorem 1 to give 
the asymptotic version of the expected values in (9.13). Hence,

$$
\begin{aligned}
& M \sim \int_{-\frac{\pi}{2}}^{0} \frac{f(\theta)}{\sqrt{\nu|\sin \theta|}}\left\{f_{\tilde{\phi}}\left(-\frac{\pi}{2}\right) e^{j\left(2 \pi \nu|\sin \theta|-\frac{\pi}{4}\right)}\right\} d \theta \\
& +\int_{-\frac{\pi}{2}}^{0} \frac{f(\theta)}{\sqrt{\nu|\sin \theta|}}\left\{f_{\tilde{\phi}}\left(\frac{\pi}{2}\right) e^{-j\left(2 \pi \nu|\sin \theta|-\frac{\pi}{4}\right)}\right\} d \theta \\
& +\int_{0}^{\frac{\pi}{2}} \frac{f(\theta)}{\sqrt{\nu|\sin \theta|}}\left\{f_{\tilde{\phi}}\left(-\frac{\pi}{2}\right) e^{j\left(2 \pi \nu|\sin \theta|-\frac{\pi}{4}\right)}\right\} d \theta \\
& +\int_{0}^{\frac{\pi}{2}} \frac{f(\theta)}{\sqrt{\nu|\sin \theta|}}\left\{f_{\tilde{\phi}}\left(\frac{\pi}{2}\right) e^{-j\left(2 \pi \nu|\sin \theta|-\frac{\pi}{4}\right)}\right\} d \theta .
\end{aligned}
$$

Substituting $f_{\tilde{\tilde{\phi}}}\left(-\frac{\pi}{2}\right)=f_{\phi}(\Delta), f_{\tilde{\tilde{\phi}}}\left(\frac{\pi}{2}\right)=f_{\phi}(\Delta-\pi), f_{\tilde{\phi}}\left(-\frac{\pi}{2}\right)=f_{\phi}(\Delta-\pi)$, and $f_{\tilde{\phi}}\left(\frac{\pi}{2}\right)=f_{\phi}(\Delta)$ into (9.14), we have

$$
M \sim \frac{f_{\phi}(\Delta) e^{-j \frac{\pi}{4}}}{\sqrt{\nu}} I_{1}+\frac{f_{\phi}(\Delta-\pi) e^{j \frac{\pi}{4}}}{\sqrt{\nu}} I_{2}+\frac{f_{\phi}(\Delta-\pi) e^{-j \frac{\pi}{4}}}{\sqrt{\nu}} I_{3}+\frac{f_{\phi}(\Delta) e^{j \frac{\pi}{4}}}{\sqrt{\nu}} I_{4},
$$

where $I_{1}^{*}=I_{2}=\int_{-\frac{\pi}{2}}^{0} \frac{f(\theta) e^{j 2 \pi \nu \sin \theta}}{\sqrt{|\sin \theta|}} d \theta$ and $I_{3}^{*}=I_{4}=\int_{0}^{\frac{\pi}{2}} \frac{f(\theta) e^{-j 2 \pi \nu \sin \theta}}{\sqrt{\sin \theta}} d \theta$. Hence, we only need to compute $I=\int_{0}^{\frac{\pi}{2}} \frac{g(\theta) e^{-j 2 \pi \nu \sin \theta}}{\sqrt{\sin \theta}} d \theta$, where $g(\theta)=f(\theta)$ for $I_{3}$ and $I_{4}$ and $g(\theta)=f(-\theta)$ for $I_{1}$ and $I_{2}$. Let $X=\sin \theta$ and rewrite $I$ as

$$
I=\int_{0}^{1} \frac{g\left(\sin ^{-1}(x)\right) e^{-j 2 \pi \nu x}}{\sqrt{x} \sqrt{1-x^{2}}} d x=\int_{0}^{1} e^{-j 2 \pi \nu x} q(x) d x
$$

where $q(x)=\frac{g\left(\sin ^{-1}(x)\right)}{\sqrt{x} \sqrt{1-x^{2}}}[H(x)-H(x-1)]$. As in Theorem $1, q(x)$ has its worst singularities at $x \in\{0,1\}$. Using [155, p. 55] again, we have $q(x) \sim$ $F_{1}(x)=g(0) H(x)|x|^{-\frac{1}{2}}$, when $x \longrightarrow 0$, and $q(x) \sim F_{2}(x)=-\frac{1}{\sqrt{2}} g\left(\frac{\pi}{2}\right)|x-1|^{-\frac{1}{2}}+$ $\frac{1}{\sqrt{2}} g\left(\frac{\pi}{2}\right)|x-1|^{-\frac{1}{2}} H(x-1)$, when $x \longrightarrow 1$. Thus, according to [155, Theorem 19, p. 52], we have $I \sim G_{1}(y)+G_{2}(y)$, where $G_{i}(\cdot)$ is the Fourier transform of $F_{i}(\cdot)$ and $y=\nu / 2 \pi$. Hence, 


$$
I \sim g(0) \frac{e^{-j \frac{\pi}{4} \operatorname{sgn}(y)}}{\sqrt{2}}|y|^{-\frac{1}{2}}-g\left(\frac{\pi}{2}\right) \frac{e^{-j 2 \pi y}}{\sqrt{2}}|y|^{-\frac{1}{2}}+g\left(\frac{\pi}{2}\right) e^{-j 2 \pi y} \frac{e^{-j \frac{\pi}{4} \operatorname{sgn}(y)}}{2}|y|^{-\frac{1}{2}}
$$

Substituting $\nu=2 \pi y$ in (9.17) and nothing that $\nu>0$ gives

$$
I \sim \frac{g(0) e^{-j \frac{\pi}{4}}}{\sqrt{2 \nu}}-\frac{g\left(\frac{\pi}{2}\right) e^{-j 2 \pi \nu}}{\sqrt{2 \nu}}+\frac{g\left(\frac{\pi}{2}\right) e^{-j 2 \pi \nu} e^{-j \frac{\pi}{4}}}{2 \sqrt{\nu}}=\frac{1}{\sqrt{\nu}}\left\{g(0) e^{-j \frac{\pi}{4}}-\frac{g\left(\frac{\pi}{2}\right) e^{-j 2 \pi \nu+j \frac{\pi}{4}}}{2}\right\}
$$

Substituting (9.18) into (9.15) we get

$$
\begin{aligned}
M & \sim \frac{f_{\phi}(\Delta) e^{-j \frac{\pi}{4}}}{\nu}\left\{\frac{f_{\theta}(0) e^{j \frac{\pi}{4}}}{\sqrt{2}}-\frac{f_{\theta}\left(-\frac{\pi}{2}\right)}{2} e^{j\left(2 \pi \nu-\frac{\pi}{4}\right)}\right\} \\
& +\frac{f_{\phi}(\Delta-\pi) e^{j \frac{\pi}{4}}}{\nu}\left\{\frac{f_{\theta}(0) e^{-j \frac{\pi}{4}}}{\sqrt{2}}-\frac{f_{\theta}\left(-\frac{\pi}{2}\right) e^{-j\left(2 \pi \nu-\frac{\pi}{4}\right)}}{2}\right\} \\
& +\frac{f_{\phi}(\Delta-\pi) e^{-j \frac{\pi}{4}}}{\nu}\left\{\frac{f_{\theta}(0) e^{j \frac{\pi}{4}}}{\sqrt{2}}-\frac{f_{\theta}\left(\frac{\pi}{2}\right) e^{j\left(2 \pi \nu-\frac{\pi}{4}\right)}}{2}\right\} \\
& +\frac{f_{\phi}(\Delta) e^{j \frac{\pi}{4}}}{\nu}\left\{\frac{f_{\theta}(0) e^{-j \frac{\pi}{4}}}{\sqrt{2}}-\frac{f_{\theta}\left(\frac{\pi}{2}\right)}{2} e^{-j\left(2 \pi \nu-\frac{\pi}{4}\right)}\right\}, \\
& =\frac{j}{2 \nu}\left\{-f_{\phi}(\Delta-\pi) f_{\theta}\left(-\frac{\pi}{2}\right) e^{-j 2 \pi \nu}\right\} \\
& +\frac{j}{2 \nu}\left\{f_{\phi}(\Delta-\pi) f_{\theta}\left(\frac{\pi}{2}\right) e^{j 2 \pi \nu}\right\}+\frac{j}{2 \nu}\left\{f_{\phi}(\Delta) f_{\theta}\left(-\frac{\pi}{2}\right) e^{j 2 \pi \nu}\right\}, \\
& -\frac{j}{2 \nu}\left\{f_{\phi}(\Delta) f_{\theta}\left(\frac{\pi}{2}\right) e^{-j 2 \pi \nu}\right\}+\frac{\sqrt{2} f_{\theta}(0)}{\nu}\left(f_{\phi}(\Delta)+f_{\phi}(\Delta-\pi)\right) \triangleq \frac{\alpha(\nu, \Delta)}{\nu} .
\end{aligned}
$$

According to a two-dimensional version of the integral test (a one-dimensional version of the integral test can be found in [156, Theorem 9.10, p. 619]), (7.21) converges if and only if the following expression converges as $N_{x} \rightarrow \infty$, 
$N_{y} \rightarrow \infty$

$$
Z_{0}=\int_{-N_{x}}^{N_{x}} \int_{y \in \mathcal{Y}}\left(1-\frac{|x|}{N_{x}}\right)\left(1-\frac{|y|}{N_{y}}\right)\left|M_{x, y}\right|^{2} d y d x .
$$

where $\mathcal{Y}=\left(-N_{y},-1\right) \cup\left(1, N_{y}\right)$. Note that the interval $[-1,+1]$ has been cut out of the integration zone for $y$. This is valid as it simply reflects that the first few terms indexed by $w$ in (7.21) are finite. As $M_{x, y} \sim \frac{\alpha(\nu, \Delta)}{\nu}$, we have

$$
Z_{0} \sim \int_{-N_{x}}^{N_{x}} \int_{y \in \mathcal{Y}}\left(1-\frac{|x|}{N_{x}}\right)\left(1-\frac{|y|}{N_{y}}\right) \frac{|\alpha(\nu, \Delta)|^{2}}{x^{2} d_{x}^{2}+y^{2} d_{y}^{2}} d y d x
$$

Since $|\alpha(\nu, \Delta)|^{2}$ is bounded and non-vanishing, the limit of $Z_{0}$ exists if and only if the limit of $Z_{1}$ exists, where

$$
\begin{aligned}
Z_{1} & \sim \int_{-N_{x}}^{N_{x}} \int_{y \in \mathcal{Y}}\left(1-\frac{|x|}{N_{x}}\right)\left(1-\frac{|y|}{N_{y}}\right) \frac{1}{x^{2}+y^{2}} d y d x \\
& =4 \int_{0}^{N_{x}} \int_{1}^{N_{y}}\left(1-\frac{x}{N_{x}}\right)\left(1-\frac{y}{N_{y}}\right) \frac{1}{x^{2}+y^{2}} d y d x=I_{A}+I_{B}+I_{C}+I_{D}
\end{aligned}
$$

where $I_{A}=\int_{0}^{N_{x}} \int_{1}^{N_{y}} \frac{1}{x^{2}+y^{2}} d y d x, I_{B}=-\int_{0}^{N_{x}} \int_{1}^{N_{y}} \frac{1}{N_{x}} \frac{x}{x^{2}+y^{2}} d y d x$, $I_{C}=\int_{0}^{N_{x}} \int_{1}^{N_{y}} \frac{1}{N_{x} N_{y}} \frac{x y}{x^{2}+y^{2}} d y d x$, and $I_{D}=-\int_{0}^{N_{x}} \int_{1}^{N_{y}} \frac{1}{N_{y}} \frac{y}{x^{2}+y^{2}} d y d x$. Using the integral result $\int_{0}^{\infty}\left(x^{2}+y^{2}\right)^{-1} d x=\pi / 2 y$ [130, Eq. 2.124.1, p. 71], we are able to investigate the limits of $I_{A}, I_{B}, I_{C}$ and $I_{D}$. First, we consider $I_{D}$ :

$\lim _{N_{x} \rightarrow \infty, N_{y} \rightarrow \infty} I_{D}=-\lim _{N_{y} \rightarrow \infty} \int_{1}^{N_{y}} \frac{y}{N_{y}} \int_{0}^{\infty} \frac{y}{x^{2}+y^{2}} d x d y=-\lim _{N_{y} \rightarrow \infty} \int_{1}^{N_{y}} \frac{\pi}{2 N_{y}} d y=-\frac{\pi}{2}$,

which is finite. Similarly, $I_{B}$ and $I_{C}$ are also finite in the limit. Thus, the limiting behavior of (7.21) depends on $I_{A}$, and we have $\lim _{N_{x} \rightarrow \infty, N_{y} \rightarrow \infty} I_{A}=\lim _{N_{y} \rightarrow \infty} \int_{1}^{N_{y}} \int_{0}^{\infty} \frac{1}{x^{2}+y^{2}} d x d y=\lim _{N_{y} \rightarrow \infty} \int_{1}^{N_{y}} \frac{\pi}{2 y} d y=\lim _{N_{y} \rightarrow \infty} \frac{\pi}{2} \log \left(N_{y}\right)$. 
Hence, $I_{A}$ grows logarithmically and (7.21) grows logarithmically as desired.

\subsection{Derivation of $\mu_{\text {UPA }}$ for Uniform Angular Distri- butions}

In this scenario, beginning with the basic definition of $\mu_{\mathrm{UPA}}$, we have

$$
\begin{aligned}
\mu_{\mathrm{UPA}} & =\frac{1}{N} \mathbb{E}\left[\left|\mathbf{a}_{i r}^{\mathrm{H}} \mathbf{a}_{j s}\right|^{2}\right] \\
& =\frac{1}{N} \sum_{k=1}^{N} \sum_{l=1}^{N} \mathbb{E}\left[a_{i r k}^{*} a_{j s k} a_{j s l}^{*} a_{i r l}\right] \\
& =\frac{1}{N} \sum_{k=1}^{N} \sum_{l=1}^{N} \mathbb{E}\left[a_{i r k}^{*} a_{i r l}\right] \mathbb{E}\left[a_{j s l}^{*} a_{j s k}\right], \\
& =\frac{1}{N} \sum_{k=1}^{N} \sum_{l=1}^{N}\left|\mathbb{E}\left[a_{i r k}^{*} a_{i r l}\right]\right|^{2} \\
& =\frac{1}{N} \sum_{k_{x}=1}^{N_{x}} \sum_{k_{y}=1}^{N_{y}} \sum_{l_{x}=1}^{l_{x}} \sum_{l_{y}=1}^{N_{y}}\left|\mathbb{E}\left[a_{i r x k_{x}}^{*} a_{i r y k_{y}}^{*} a_{i r x l_{x}} a_{i r y l_{y}}\right]\right|^{2} .
\end{aligned}
$$

The expectation in (9.23) is given by

$$
I_{1}=\mathbb{E}\left[\exp \left\{j 2 \pi \sin \theta\left(d_{y}\left(k_{y}-l_{y}\right) \sin \phi+d_{x}\left(k_{x}-l_{x}\right) \cos \phi\right)\right\}\right],
$$

Taking expectation over $\phi$ first gives an integral of the form

$$
\begin{aligned}
& \frac{1}{2 \pi} \int_{0}^{2 \pi} \exp \{j z(\alpha \sin \phi+\beta \cos \phi)\} d \phi \\
& =\frac{1}{2 \pi} \int_{0}^{2 \pi} \exp \left\{j z\left(\sqrt{\alpha^{2}+\beta^{2}} \sin (\phi+\Delta)\right)\right\} d \phi \\
& =J_{0}\left(z \sqrt{\alpha^{2}+\beta^{2}}\right),
\end{aligned}
$$


where $\Delta=\tan ^{-1}(\beta / \alpha)$. Hence,

$$
\begin{aligned}
I_{1} & =\mathbb{E}\left[J_{0}\left(2 \pi \sin \theta \sqrt{d_{y}^{2}\left(k_{y}-l_{y}\right)^{2}+d_{x}^{2}\left(k_{x}-l_{x}\right)^{2}}\right)\right], \\
& =\frac{1}{\pi} \int_{0}^{\pi} J_{0}(\chi \sin \theta) d \theta
\end{aligned}
$$

where $\chi=2 \pi \sqrt{d_{y}^{2}\left(k_{y}-l_{y}\right)^{2}+d_{x}^{2}\left(k_{x}-l_{x}\right)^{2}}$.

From [130, Eq.6, p. 724], we have

$$
\int_{0}^{\pi} J_{0}(2 z \sin x) \cos (2 n x) d x=\pi J_{n}^{2}(x)
$$

Thus

$$
\int_{0}^{\pi} J_{0}(2 z \sin x) d x=\pi J_{0}^{2}(z)
$$

and we can rewrite $I_{1}$ as

$$
I_{1}=J_{0}^{2}\left(\pi \sqrt{d_{y}^{2}\left(k_{y}-l_{y}\right)^{2}+d_{x}^{2}\left(k_{x}-l_{x}\right)^{2}}\right) .
$$

Then, substituting $I_{1}$ into (9.23) allows $\mu_{\mathrm{UPA}}$ to be written as in (7.22).

\subsection{Logarithmic growth of $\mu_{\text {UPA }}$ for Uniform Angu- lar Distributions}

A simple change of indices, $m=r_{y}-s_{y}, n=r_{x}-s_{x}$ in (7.22) gives

$$
\mu_{\mathrm{UPA}}=\sum_{m=1-N_{y}}^{N_{y}-1} \sum_{n=1-N_{x}}^{N_{x}-1}\left(1-\frac{|m|}{N_{y}}\right)\left(1-\frac{|n|}{N_{x}}\right) J_{0}^{4}(A(m, n)),
$$


where $A(m, n)=\pi \sqrt{m^{2} d_{y}^{2}+n^{2} d_{x}^{2}}$. The sum in (9.31) is dominated by

$$
\mu_{1}=\sum_{m=1-N_{y}}^{N_{y}-1} \sum_{n=1-N_{x}}^{N_{x}-1} J_{0}^{4}(A(m, n))
$$

and it is straightforward to show that the remaining terms in (9.31) are finite as $N \rightarrow \infty$. Hence, the asymptotic behavior of $\mu_{U P A}$ is the same as for $\mu_{1}$. Similarly, the sum in (9.32) is dominated by

$$
\mu_{2}=4 \sum_{m=1}^{N_{y}-1} \sum_{n=1}^{N_{x}-1} J_{0}^{4}(A(m, n))
$$

using the fact that $A(m, n)$ is an even function of $m$ and $n$ and neglecting terms which can be shown to be finite. Again, the asymptotic behavior of $\mu_{\mathrm{UPA}}$ is the same as for $\mu_{2}$. Using the asymptotic equivalence [129, Eq. 10.17.3], $J_{0}(z) \sim \sqrt{\frac{2}{\pi z}} \cos (z-\pi / 4)$, we see that $\mu_{\mathrm{UPA}}$ behaves like

$$
\mu_{3}=16 \sum_{m=1}^{N_{y}-1} \sum_{n=1}^{N_{x}-1} \frac{\cos ^{4}(A(m, n)-\pi / 4)}{\pi^{2} A^{2}(m, n)}=\frac{16}{\pi^{4}} \sum_{m=1}^{N_{y}-1} \sum_{n=1}^{N_{x}-1} \frac{\cos ^{4}(A(m, n)-\pi / 4)}{m^{2} d_{y}^{2}+n^{2} d_{x}^{2}} .
$$

Application of a two-dimensional version of the integral test (one-dimensional version of the integral test can be found in [156, Theorem 9.10, p. 619]) and some further analysis to handle the oscillations in the $\cos ^{4}(\cdot)$ function via upper and lower bounds shows that the asymptotic behavior of $\mu_{3}$ is the same as that of

$$
\mu_{4}=\int_{1}^{N_{y}-1} \int_{1}^{N_{x}-1} \frac{1}{x^{2} d_{x}^{2}+y^{2} d_{y}^{2}} d_{x} d_{y}=\frac{1}{d_{x} d_{y}} \int_{d_{y}}^{\left(N_{y}-1\right) d_{y}} \int_{d_{x}}^{\left(N_{x}-1\right) d_{x}} \frac{1}{u^{2}+v^{2}} d u d v
$$


Converting to polar coordinates, a simple upper bound on (9.35) is

$$
\mu_{4} \leqslant \int_{0}^{\pi / 2} \int_{\rho_{\min }}^{\rho_{\max }} \frac{1}{\rho^{2}} \rho d \rho d \theta=\frac{\pi}{2}\left(\log \left(\rho_{\max }\right)-\log \left(\rho_{\min }\right)\right)
$$

where $\rho_{\min }=\min \left(d_{x}, d_{y}\right)$ and $\rho_{\max }=\sqrt{2} \max \left(\left(N_{x}-1\right) d_{x},\left(N_{y}-1\right) d_{y}\right)$. Hence, the upper limit on $\mu_{4}$ grows logarithmically with $N$ as $N^{1 / 2} \leqslant \max \left(N_{x}, N_{y}\right) \leqslant$ $N$, so that $\frac{1}{2} \log N \leqslant \log \left(\max \left(N_{x}, N_{y}\right)\right) \leqslant \log N$. Similarly, when $N_{x} \rightarrow \infty$, $N_{y} \rightarrow \infty$ as $N \rightarrow \infty, \mu_{4}$ can be lower bounded by a logarithmic function of $N$ by integrating over a sector of an annulus contained inside the integration region of (9.35). Hence, $\mu_{\mathrm{UPA}}$ grows logarithmically with $N$ as required. 


\section{Bibliography}

[1] M. Series, "IMT Vision-framework and overall objectives of the future development of IMT for 2020 and beyond," Recommendation ITU, pp. 2083-0, 2015.

[2] J. G. Andrews, S. Buzzi, W. Choi, S. V. Hanly, A. Lozano, A. C. Soong, and J. C. Zhang, “What will 5G be?," IEEE J. Sel. Areas Commun., vol. 32, no. 6, pp. 1065-1082, 2014.

[3] E. G. Larsson, O. Edfors, F. Tufvesson, and T. L. Marzetta, "Massive MIMO for next generation wireless systems," IEEE Commun. Mag., vol. 52, pp. 186-195, Feb. 2014.

[4] L. Zheng and D. N. C. Tse, "Diversity and multiplexing: A fundamental tradeoff in multiple-antenna channels," IEEE Trans. Inf. Theory, vol. 49, no. 5, pp. 1073-1096, 2003.

[5] R. Couillet and M. Debbah, Random Matrix Methods for Wireless Communications. Cambridge University Press, 2011.

[6] H. Cramér, Random Variables and Probability Distributions, vol. 36. Cambridge University Press, 2004. 
[7] F. Rusek, D. Persson, B. K. Lau, E. G. Larsson, T. L. Marzetta, O. Edfors, and F. Tufvesson, "Scaling up MIMO: Opportunities and challenges with very large arrays," IEEE Signal Process. Mag., vol. 30, pp. 40-60, Jan. 2013.

[8] X. Gao, O. Edfors, F. Rusek, and F. Tufvesson, "Massive MIMO performance evaluation based on measured propagation data," IEEE Trans. Wireless Commun., vol. 14, no. 7, pp. 3899-3911, 2015.

[9] H. Q. Ngo, E. G. Larsson, and T. L. Marzetta, "Aspects of favorable propagation in massive MIMO," in Proc. IEEE EUSIPCO, pp. 76-80, Sep. 2014.

[10] B. M. Hochwald, T. L. Marzetta, and V. Tarokh, “Multiple-antenna channel hardening and its implications for rate feedback and scheduling," IEEE Trans. Inf. Theory, vol. 50, no. 9, pp. 1893-1909, 2004.

[11] R. W. Heath, N. Gonzalez-Prelcic, S. Rangan, W. Roh, and A. M. Sayeed, "An overview of signal processing techniques for millimeter wave MIMO systems," IEEE J. Sel. Topics Signal Process., vol. 10, no. 3, pp. 436453, 2016.

[12] A. Alkhateeb, J. Mo, N. González-Prelcic, and R. W. Heath, “MIMO precoding and combining solutions for millimeter-wave systems," IEEE Commun. Mag., vol. 52, no. 12, pp. 122-131, 2014.

[13] M. K. Karakayali, G. J. Foschini, and R. A. Valenzuela, “Network coordination for spectrally efficient communications in cellular systems," IEEE Trans. Wireless Commun., vol. 13, no. 4, pp. 56-61, 2006.

[14] A. Lozano, R. W. Heath, and J. G. Andrews, "Fundamental limits of cooperation," IEEE Trans. Inf. Theory, vol. 59, no. 9, pp. 5213-5226, 2013. 
[15] T. Baykas, C.-S. Sum, Z. Lan, J. Wang, M. A. Rahman, H. Harada, and S. Kato, "IEEE 802.15. 3c: The first IEEE wireless standard for data rates over 1 Gb/s," IEEE Commun. Mag., vol. 49, no. 7, pp. 114-121, 2011.

[16] Specifications, "IEEE P802. 11ad TM /D8D9. 05 Draft Standard for Information Technology-6 Telecommunications and Information Exchange Between 7 Systems-Local and Metropolitan Area Networks-Specific 8 Requirements-9," 2012.

[17] J. Wang, “Beam codebook based beamforming protocol for multi-Gbps millimeter-wave WPAN systems," IEEE J. Sel. Areas Commun., vol. 27, no. 8, pp. 1390-1399, 2009.

[18] S. Hur, T. Kim, D. J. Love, J. V. Krogmeier, T. A. Thomas, and A. Ghosh, "Millimeter wave beamforming for wireless backhaul and access in small cell networks," IEEE Trans. Commun., vol. 61, no. 10, pp. 4391$4403,2013$.

[19] G. H. Song, J. Brady, and A. M. Sayeed, "Beamspace MIMO transceivers for low-complexity and near-optimal communication at mm-wave frequencies.," in Proc. IEEE ICASSP, pp. 4394-4398, 2013.

[20] X. Zhang, A. F. Molisch, and S.-Y. Kung, “Variable-phase-shift-based RF-baseband codesign for MIMO antenna selection," IEEE J. Sel. Topics Signal Process., vol. 53, no. 11, pp. 4091-4103, 2005.

[21] J. Nsenga, W. Van Thillo, F. Horlin, V. Ramon, A. Bourdoux, and R. Lauwereins, "Joint transmit and receive analog beamforming in 60 GHz MIMO multipath channels," in Proc. IEEE ICC, pp. 1-5, 2009.

[22] K. T. Truong and R. W. Heath, "The viability of distributed antennas for massive MIMO systems," in Proc. Asilomar, pp. 1318-1323, 2013. 
[23] J. Zhang, X. Yuan, and L. Ping, "Hermitian precoding for distributed MIMO systems with individual channel state information," IEEE J. Sel. Areas Commun., vol. 31, no. 2, pp. 241-250, 2013.

[24] M. Matthaiou, C. Zhong, M. R. McKay, and T. Ratnarajah, "Sum rate analysis of ZF receivers in distributed MIMO systems," IEEE J. Sel. Areas Commun., vol. 31, no. 2, pp. 180-191, 2013.

[25] H. Yin, D. Gesbert, and L. Cottatellucci, "Dealing with interference in distributed large-scale MIMO systems: A statistical approach," IEEE J. Sel. Topics Signal Process., vol. 8, no. 5, pp. 942-953, 2014.

[26] C. Masouros, M. Sellathurai, and T. Ratnarajah, "Large-scale MIMO transmitters in fixed physical spaces: The effect of transmit correlation and mutual coupling," IEEE Trans. Commun., vol. 61, no. 7, pp. 27942804, 2013.

[27] P. J. Smith, C. Neil, M. Shafi, and P. A. Dmochowski, "On the convergence of massive MIMO systems," in Proc. IEEE ICC, pp. 5191-5196, 2014.

[28] X. Gao, O. Edfors, F. Rusek, and F. Tufvesson, "Linear pre-coding performance in measured very-large MIMO channels," in Proc. IEEE VTC Fall, pp. 1-5, 2011.

[29] S. Payami and F. Tufvesson, "Channel measurements and analysis for very large array systems at $2.6 \mathrm{GHz}$," in Proc. EUCAP, pp. 433-437, 2012.

[30] X. Li, L. Qiu, and Y. Dong, "Asymptotic equivalent performance of multicell massive MIMO with spatial-temporal correlation," IEEE Commun. Lett., vol. 20, no. 3, pp. 518-521, 2016. 
[31] H. Sifaou, A. Kammoun, L. Sanguinetti, M. Debbah, and M.-S. Alouini, "Max-min SINR in large-scale single-cell MU-MIMO: Asymptotic analysis and low-complexity transceivers," IEEE Trans. Signal Process., vol. 65 , no. 7, pp. 1841-1854, 2017.

[32] K. A. Alnajjar, P. J. Smith, and G. K. Woodward, "Performance of massive MIMO V-BLAST with channel correlation and imperfect CSI," in Proc. ATNAC, pp. 170-175, 2014.

[33] R. Senanayake, P. L. Yeoh, and J. S. Evans, "Performance analysis of centralized and partially decentralized co-operative networks," IEEE Trans. Commun., vol. 64, no. 2, pp. 863-875, 2015.

[34] J. Hoydis, S. Ten Brink, and M. Debbah, "Massive MIMO in the UL/DL of cellular networks: How many antennas do we need?," IEEE J. Sel. Areas Commun., vol. 31, no. 2, pp. 160-171, 2013.

[35] C. T. Neil et al., "Impact of microwave and mmWave channel models on 5G systems performance," IEEE Trans. Antennas Propag., vol. 65, pp. 6505-6520, Dec. 2017.

[36] S. Sangodoyin, V. Kristem, C. Bas, M. Käske, J. Lee, C. Schneider, G. Sommerkorn, J. Zhang, R. Thomä, and A. Molisch, "Cluster characterization of 3D MIMO propagation channel in an urban macrocellular environment," IEEE Trans. Wireless Commun., vol. 17, no. 8, pp. 50765091, 2018.

[37] M. R. Akdeniz, Y. Liu, M. K. Samimi, S. Sun, S. Rangan, T. S. Rappaport, and E. Erkip, “Millimeter wave channel modeling and cellular capacity evaluation," IEEE J. Sel. Areas Commun., vol. 32, pp. 1164-1179, Jun. 2014. 
[38] H. Q. Ngo, E. G. Larsson, and T. L. Marzetta, "Energy and spectral efficiency of very large multiuser MIMO systems," IEEE Trans. Commun., vol. 61, pp. 1436-1449, Apr. 2013.

[39] S. Kutty and D. Sen, "Beamforming for millimeter wave communications: An inclusive survey," IEEE Commun. Surveys Tuts, vol. 18, no. 2, pp. 949-973, 2016.

[40] A. Goldsmith, Wireless Communications. Cambridge University Press, 2005.

[41] T. S. Rappaport, S. Sun, R. Mayzus, H. Zhao, Y. Azar, K. Wang, G. N. Wong, J. K. Schulz, M. Samimi, and F. Gutierrez, "Millimeter wave mobile communications for $5 \mathrm{G}$ cellular: It will work!," IEEE Access, vol. 1, pp. 335-349, 2013.

[42] M. R. Akdeniz, Y. Liu, M. K. Samimi, S. Sun, S. Rangan, T. S. Rappaport, and E. Erkip, "Millimeter wave channel modeling and cellular capacity evaluation," IEEE J. Sel. Areas Commun., vol. 32, no. 6, pp. 1164-1179, 2014.

[43] D. Tse and P. Viswanath, Fundamentals of Wireless Communication. Cambridge University Press, 2005.

[44] G. J. Foschini and M. J. Gans, "On limits of wireless communications in a fading environment when using multiple antennas," Wireless personal communications, vol. 6, no. 3, pp. 311-335, 1998.

[45] E. Telatar, "Capacity of multi-antenna Gaussian channels," European transactions on telecommunications, vol. 10, no. 6, pp. 585-595, 1999. 
[46] T. L. Marzetta et al., "Noncooperative cellular wireless with unlimited numbers of base station antennas," IEEE Trans. Wireless Commun., vol. 9, no. 11, p. 3590, 2010.

[47] B. Clerckx and C. Oestges, MIMO Wireless Networks: Channels, Techniques and Standards for Multi-antenna, Multi-user and Multi-cell systems. Academic Press, 2013.

[48] A. A. Saleh and R. Valenzuela, "A statistical model for indoor multipath propagation," IEEE J. Sel. Areas Commun., vol. 5, pp. 128-137, Feb. 1987.

[49] S. Buzzi, C. D’Andrea, T. Foggi, A. Ugolini, and G. Colavolpe, "Single-carrier modulation versus OFDM for millimeter-wave wireless MIMO," IEEE Trans. Commun, vol. 66, no. 3, pp. 1335-1348, 2017.

[50] C. Bluemm, M. Schaedler, M. Kuschnerov, F. Pittala, and C. Xie, "Single carrier vs. OFDM for coherent $600 \mathrm{~Gb} / \mathrm{s}$ data centre interconnects with nonlinear equalization," in IEEE OFC, pp. 1-3, IEEE, 2019.

[51] M. Wu, D. Wubben, A. Dekorsy, P. Baracca, V. Braun, and H. Halbauer, “On OFDM and SC-FDE transmissions in millimeter wave channels with beamforming," in 2016 IEEE VTC Spring, pp. 1-5, 2016.

[52] R. J. Muirhead, Aspects of Multivariate Statistical Theory, vol. 197. John Wiley \& Sons, 2009.

[53] S. L. Loyka, "Channel capacity of MIMO architecture using the exponential correlation matrix," IEEE Commun. Lett., vol. 5, no. 9, pp. 369$371,2001$.

[54] M. Chiani, M. Z. Win, and A. Zanella, “On the capacity of spatially correlated MIMO Rayleigh-fading channels," IEEE Trans. Inf. Theory, vol. 49, no. 10, pp. 2363-2371, 2003. 
[55] R. Clarke, "A Statistical Theory of Mobile-radio Reception," Bell system technical journal, vol. 47, no. 6, pp. 957-1000, 1968.

[56] W. C. Jakes and D. C. Cox, Microwave mobile communications. Wiley-IEEE Press, 1994.

[57] D.-S. Shiu, G. J. Foschini, M. J. Gans, and J. M. Kahn, “Fading correlation and its effect on the capacity of multielement antenna systems," IEEE Trans. Commun., vol. 48, no. 3, pp. 502-513, 2000.

[58] J. Nam, G. Caire, and J. Ha, "On the role of transmit correlation diversity in multiuser MIMO systems," IEEE Trans. Inf. Theory, vol. 63, no. 1, pp. 336-354, 2017.

[59] A. Adhikary, J. Nam, J.-Y. Ahn, and G. Caire, "Joint spatial division and multiplexing - the large-scale array regime," IEEE Trans. Inf. Theory, vol. 59, no. 10, pp. 6441-6463, 2013.

[60] Z. Jiang, A. F. Molisch, G. Caire, and Z. Niu, “Achievable rates of FDD massive MIMO systems with spatial channel correlation," IEEE Trans. Wireless Commun., vol. 14, no. 5, pp. 2868-2882, 2015.

[61] A. F. Molisch, Wireless Communications, vol. 34. John Wiley \& Sons, 2012.

[62] J. Fuhl, A. F. Molisch, and E. Bonek, “Unified channel model for mobile radio systems with smart antennas," IEE Proceedings-Radar, Sonar and Navigation, vol. 145, no. 1, pp. 32-41, 1998.

[63] A. Forenza, D. J. Love, and R. W. Heath, "Simplified spatial correlation models for clustered MIMO channels with different array configurations," IEEE Trans. Veh. Technol., vol. 56, no. 4, pp. 1924-1934, 2007. 
[64] D. J. Love, R. W. Heath Jr, V. K. Lau, D. Gesbert, B. D. Rao, and M. Andrews, "An overview of limited feedback in wireless communication systems," IEEE J. Sel. Areas Commun., vol. 26, no. 8, pp. 1341-1365, 2008.

[65] P. Harris, S. Malkowsky, J. Vieira, E. Bengtsson, F. Tufvesson, W. B. Hasan, L. Liu, M. Beach, S. Armour, and O. Edfors, "Performance characterization of a real-time massive MIMO system with LOS mobile channels," IEEE J. Sel. Areas Commun., vol. 35, no. 6, pp. 1244-1253, 2017.

[66] D. Mi, M. Dianati, L. Zhang, S. Muhaidat, and R. Tafazolli, "Massive MIMO performance with imperfect channel reciprocity and channel estimation error," IEEE Trans. Commun, vol. 65, no. 9, pp. 3734-3749, 2017.

[67] S. Sun, T. S. Rappaport, R. W. Heath, A. Nix, and S. Rangan, "MIMO for millimeter-wave wireless communications: Beamforming, spatial multiplexing, or both?," IEEE Commun. Mag., vol. 52, no. 12, pp. 110121, 2014.

[68] S. Lakshminarayana, M. Assaad, and M. Debbah, "Coordinated multicell beamforming for massive MIMO: A random matrix approach," IEEE Trans. Inf. Theory, vol. 61, pp. 3387-3412, June 2015.

[69] E. Björnson, J. Hoydis, M. Kountouris, and M. Debbah, "Massive MIMO systems with non-ideal hardware: Energy efficiency, estimation, and capacity limits," IEEE Trans. Inf. Theory, vol. 60, pp. 7112-7139, Nov 2014.

[70] G. Liu, X. Hou, F. Wang, J. Jin, H. Tong, and Y. Huang, "Achieving 3DMIMO with massive antennas from theory to practice with evaluation and field trial results," IEEE Systems Journal, vol. 11, pp. 62-71, March 2017. 
[71] Y. Saito, A. Benjebbour, Y. Kishiyama, X. Wang, X. Hou, H. Jiang, L. Lu, W. Liang, B. Li, L. Gu, Y. Cui, and T. Kashima, "Large scale field experimental trial of downlink TDD massive MIMO at the $4.5 \mathrm{GHz}$ band, " in Proc. IEEE VTC Spring, pp. 1-5, June 2017.

[72] E. G. Larsson, "Massive MIMO for 5G: Overview and the road ahead," in Proc. CISS, pp. 1-1, March 2017.

[73] M. Shafi, A. F. Molisch, P. J. Smith, T. Haustein, P. Zhu, P. De Silva, F. Tufvesson, A. Benjebbour, and G. Wunder, "5G: A tutorial overview of standards, trials, challenges, deployment, and practice," IEEE J. Sel. Areas Commun., vol. 35, no. 6, pp. 1201-1221, 2017.

[74] E. Björnson, J. Hoydis, and L. Sanguinetti, Massive MIMO Networks: Spectral, Energy, and Hardware Efficiency. Now Publishers, Inc., 2017.

[75] H. Q. Ngo and E. G. Larsson, “No downlink pilots are needed in TDD massive MIMO," IEEE Trans. Wireless Commun., vol. 16, pp. 2921-2935, May 2017.

[76] O. El Ayach, S. Rajagopal, S. Abu-Surra, Z. Pi, and R. W. Heath, "Spatially sparse precoding in millimeter wave MIMO systems," IEEE Trans. Wireless Commun., vol. 13, no. 3, pp. 1499-1513, 2014.

[77] A. Alkhateeb, O. El Ayach, G. Leus, and R. W. Heath, “Hybrid precoding for millimeter wave cellular systems with partial channel knowledge," in Proc. ITA Workshop, pp. 1-5, 2013.

[78] T. Kim, J. Park, J.-Y. Seol, S. Jeong, J. Cho, and W. Roh, “Tens of Gbps support with mmWave beamforming systems for next generation communications," in Proc. IEEE GLOBECOM, pp. 3685-3690, 2013. 
[79] Y. M. Tsang, A. S. Poon, and S. Addepalli, "Coding the beams: Improving beamforming training in mmWave communication system," in Proc. IEEE GLOBECOM, pp. 1-6, 2011.

[80] J. Lee, G.-T. Gil, and Y. H. Lee, “Exploiting spatial sparsity for estimating channels of hybrid MIMO systems in millimeter wave communications," in Proc. IEEE GLOBECOM, pp. 3326-3331, 2014.

[81] J. Mo and R. W. Heath, “Capacity analysis of one-bit quantized MIMO systems with transmitter channel state information," IEEE Trans. Signal Process., vol. 63, no. 20, pp. 5498-5512, 2015.

[82] G. Wang and G. Ascheid, "Joint pre/post-processing design for large millimeter wave hybrid spatial processing systems," in Proc. European Wireless, pp. 1-6, 2014.

[83] O. El Ayach, R. W. Heath, S. Rajagopal, and Z. Pi, “Multimode precoding in millimeter wave MIMO transmitters with multiple antenna sub-arrays," in Proc. IEEE GLOBECOM, pp. 3476-3480, 2013.

[84] W. Roh, J.-Y. Seol, J. Park, B. Lee, J. Lee, Y. Kim, J. Cho, K. Cheun, and F. Aryanfar, "Millimeter-wave beamforming as an enabling technology for 5G cellular communications: theoretical feasibility and prototype results," IEEE Commun. Mag., vol. 52, no. 2, pp. 106-113, 2014.

[85] M. S. Rahman and K. Josiam, "Low complexity RF beam search algorithms for millimeter-wave systems," in Proc. IEEE GLOBECOM, pp. 3815-3820, 2014.

[86] L. Dai, X. Gao, J. Quan, S. Han, and I. Chih-Lin, "Near-optimal hybrid analog and digital precoding for downlink mmWave massive MIMO systems," in Proc. IEEE ICC, pp. 1334-1339, 2015. 
[87] C. Kim, T. Kim, and J.-Y. Seol, "Multi-beam transmission diversity with hybrid beamforming for MIMO-OFDM systems," in Proc. IEEE GLOBECOM Workshops, pp. 61-65, 2013.

[88] F. Sohrabi and W. Yu, "Hybrid digital and analog beamforming design for large-scale MIMO systems," in Proc. IEEE ICASSP, pp. 2929-2933, 2015.

[89] M. Costa, "Writing on dirty paper (corresp.)," IEEE Trans. Inf. Theory, vol. 29, no. 3, pp. 439-441, 1983.

[90] C. B. Peel, B. M. Hochwald, and A. L. Swindlehurst, "A vectorperturbation technique for near-capacity multiantenna multiuser communication-Part I: Channel inversion and regularization," IEEE Trans. Commun., vol. 53, no. 1, pp. 195-202, 2005.

[91] B. M. Hochwald, C. B. Peel, and A. L. Swindlehurst, "A vectorperturbation technique for near-capacity multiantenna multiuser communication-Part II: Perturbation," IEEE Trans. Commun., vol. 53, no. 3, pp. 537-544, 2005.

[92] A. Firag, P. J. Smith, A. Papathanassiou, M. Shafi, D. Basnayaka, and P. Martin, "A comparison of linear transceiver techniques in multiuser MIMO broadcast systems," in Proc. AusCTW, pp. 31-36, 2012.

[93] H. Yang and T. L. Marzetta, "Performance of conjugate and zero-forcing beamforming in large-scale antenna systems," IEEE J. Sel. Areas Commun., vol. 31, no. 2, pp. 172-179, 2013.

[94] D. Hammarwall and G. Jöngren, "Precoder structure for MIMO precoding," Apr. 26 2016. US Patent App. 15/138,675. 
[95] E. Björnson, J. Hoydis, L. Sanguinetti, et al., "Massive MIMO networks: Spectral, energy, and hardware efficiency," Foundations and Trends ${ }^{\circledR}$ in Signal Processing, vol. 11, no. 3-4, pp. 154-655, 2017.

[96] T. Eng, N. Kong, and L. B. Milstein, "Comparison of diversity combining techniques for Rayleigh-fading channels," IEEE Trans. Commun., vol. 44, no. 9, pp. 1117-1129, 1996.

[97] T. Eng, N. Kong, and L. B. Milstein, "Correction to" Comparison of diversity combining techniques for Rayleigh-fading channels"," IEEE Tran.Commun, vol. 46, no. 9, p. 1111, 1998.

[98] P. O. Akuon and H. Xu, "Optimal error analysis of receive diversity schemes on arbitrarily correlated Rayleigh fading channels," IET Communications, vol. 10, no. 7, pp. 854-861, 2016.

[99] S. Ikki and M. H. Ahmed, "Performance analysis of cooperative diversity using equal gain combining (EGC) technique over Rayleigh fading channels," in Proc. IEEE ICC, pp. 5336-5341, 2007.

[100] R. K. Mallik, M. Z. Win, and J. H. Winters, "Performance of dualdiversity predetection EGC in correlated Rayleigh fading with unequal branch SNRs," IEEE Trans. Commun., vol. 50, no. 7, pp. 1041-1044, 2002.

[101] K. Sivanesan and W. Lam, "The performance of the M-ary DS/CDMA cellular system over Rayleigh-fading channel with hybrid EGC-SC scheme," in Proc. IEEE VTC Fall, vol. 6, pp. 2755-2761, 2000.

[102] S. Halunga, M. Ionescu, and O. Fratu, "Error probability evaluation for RAKE reception of DS-CDMA signals with MRC and EGC combining in Rayleigh fading," in Proc. IEEE TELSIKS, pp. 160-163, 2007. 
[103] A. Olutayo, J. Cheng, and J. Holzman, "Asymptotically tight performance bounds for equal-gain combining over a new correlated fading channel," in Proc. CWIT, pp. 1-5, 2017.

[104] Y. Song, S. D. Blostein, and J. Cheng, “Exact outage probability for equal gain combining with cochannel interference in Rayleigh fading," IEEE Trans. Wireless Commun., vol. 2, no. 5, pp. 865-870, 2003.

[105] Q. Zhang, S. Jin, K.-K. Wong, H. Zhu, and M. Matthaiou, "Power scaling of uplink massive MIMO systems with arbitrary-rank channel means," IEEE J. Sel. Topics Signal Process., vol. 8, no. 5, pp. 966-981, 2014.

[106] H. Tataria, P. J. Smith, L. J. Greenstein, P. A. Dmochowski, and M. Matthaiou, "Impact of line-of-sight and unequal spatial correlation on uplink MU-MIMO systems," IEEE Wireless Commun. Lett., vol. 6, no. 5, pp. 634-637, 2017.

[107] G. K. Karagiannidis, "Moments-based approach to the performance analysis of equal gain diversity in Nakagami-m fading," IEEE Trans. Commun., vol. 52, no. 5, pp. 685-690, 2004.

[108] N. C. Sagias, "Closed-form analysis of equal-gain diversity in wireless radio networks," IEEE Trans. Veh. Technol., vol. 56, no. 1, pp. 173-182, 2007.

[109] N. C. Sagias, G. K. Karagiannidis, D. A. Zogas, G. S. Tombras, and S. A. Kotsopoulos, "Average output SINR of equal-gain diversity in correlated Nakagami-m fading with cochannel interference," IEEE Trans. Wireless Commun., vol. 4, no. 4, pp. 1407-1411, 2005.

[110] N. C. Sagias, G. K. Karagiannidis, P. T. Mathiopoulos, and T. A. Tsiftsis, "On the performance analysis of equal-gain diversity receivers over 
generalized gamma fading channels," IEEE Trans. Wireless Commun., vol. 5, no. 10, pp. 2967-2975, 2006.

[111] H. Fu, M. Crussière, and M. Hélard, "BER analysis for equal gain transmission in downlink multiuser MIMO systems," IEEE Wireless Commun. Lett., vol. 4, no. 5, pp. 533-536, 2015.

[112] W. Tan, S. Jin, and J. Yuan, "Spectral and energy efficiency of downlink MU-MIMO systems with MRT," China Commun., vol. 14, no. 5, pp. 105$111,2017$.

[113] W. Tan, P. J. Smith, H. A. Suraweera, M. Matthaiou, and S. Jin, "Spectral efficiency of multi-user mmWave systems with uniform linear arrays and MRT," in Proc. IEEE VTC Spring, pp. 1-5, 2016.

[114] R. Ghaffar and R. Knopp, “Diversity analysis of equal gain transmission for singleuser and multiuser MIMO," in Proc. IEEE GLOBECOM, pp. 1$5,2010$.

[115] C. Kong, C. Zhong, M. Matthaiou, and Z. Zhang, "Performance of downlink massive MIMO in Ricean fading channels with ZF precoder," in Proc. IEEE ICC, pp. 1776-1782, 2015.

[116] W. Tan, S. Jin, J. Wang, and M. Matthaiou, "Achievable sum-rate of multiuser massive MIMO downlink in Ricean fading channels," in Proc. IEEE ICC, pp. 1453-1458, 2015.

[117] K. S. Miller, Complex Stochastic Processes: An Introduction to Theory and Application. Advanced Book Program, 1974.

[118] B. Clerckx, G. Kim, and S. Kim, "Correlated fading in broadcast MIMO channels: Curse or blessing?," in IEEE GLOBECOM 2008, pp. 1-5, IEEE, 2008. 
[119] H. Tataria, P. Smith, A. Molisch, S. Sangodoyin, M. Matthaiou, P. Dmochowski, J. Zhang, and R. Thoma, "Spatial Correlation Variability in Multiuser Systems," in Proc. IEEE ICC, pp. 1-7, 2018.

[120] C. Masouros, M. Sellathurai, and T. Ratnarajah, "Large-scale MIMO transmitters in fixed physical spaces: The effect of transmit correlation and mutual coupling," IEEE Trans. Commun., vol. 61, no. 7, pp. 2794$2804,2013$.

[121] H. Tataria, P. J. Smith, P. A. Dmochowski, and M. Shafi, “General analysis of multiuser MIMO systems with regularized zero-forcing precoding under spatially correlated Rayleigh fading channels," in Proc. IEEE ICC, pp. 1-7, 2016.

[122] C. Zhang, Y. Jing, Y. Huang, and L. Yang, "Performance scaling law for multicell multiuser massive MIMO," IEEE Trans. Veh. Technol., vol. 66, no. 11, pp. 9890-9903, 2017.

[123] A. Zanella, M. Chiani, and M. Z. Win, "Performance of MIMO MRC in correlated Rayleigh fading environments," in Proc. IEEE VTC Spring, vol. 3, pp. 1633-1637, 2005.

[124] R. H. Louie, M. R. McKay, I. B. Collings, and B. Vucetic, "Capacity approximations for multiuser MIMO-MRC with antenna correlation," in Proc. IEEE ICC, pp. 5195-5200, 2007.

[125] W. Belaoura, K. Ghanem, and H. Bousbia-Salah, "Effect of spatial correlation on the ergodic capacity for downlink massive MU-MIMO systems," in Proc. ICSC, pp. 183-186, 2017.

[126] S. Li, P. J. Smith, P. A. Dmochowski, and J. Yin, "Analysis of analog and digital MRC for distributed and centralized MU-MIMO systems," IEEE Trans. Veh. Technol., vol. 68, no. 2, pp. 1948-1952, 2018. 
[127] A. M. Samoilenko, Elements of the Mathematical Theory of Multi-frequency Oscillations. Springer Science \& Business Media, 2012.

[128] H. Pishro-Nik, "Introduction to Probability, Statistics, and Random Processes," 2016.

[129] F. W. Oliver, NIST Handbook of Mathematical Functions. Cambridge University Press, 2010.

[130] I. S. Gradshteyn and I. M. Ryzhik, Table of Integrals, Series, and Products. Academic Press, 2014.

[131] A. D. Wheelon, Tables of Summable Series and Integrals Involving Bessel Functions. Holden-Day, 1968.

[132] M. Abramowitz and I. A. Stegun, Handbook of Mathematical Functions: with Formulas, Graphs, and Mathematical Tables, vol. 55. Courier Corporation, 1964.

[133] S. Li, P. J. Smith, P. A. Dmochowski, and J. Yin, "Analysis of analog and digital MRC for distributed and centralized MU-MIMO systems," IEEE Trans. Veh. Technol., vol. 68, no. 2, pp. 1948-1952, 2018.

[134] Ö. Özdogan, E. Björnson, and E. G. Larsson, “Massive MIMO with spatially correlated Rician fading channels," IEEE Trans. Commun., vol. 67, pp. 3234-3250, Jan 2019.

[135] L. Liang, W. Xu, and X. Dong, "Low-complexity hybrid precoding in massive multiuser MIMO systems," IEEE Wireless Commun. Lett., vol. 3, pp. 653-656, Dec. 2014.

[136] E. Björnson, E. G. Larsson, and T. L. Marzetta, “Massive MIMO: Ten myths and one critical question," IEEE Commun. Mag., vol. 54, pp. 114123, Feb. 2016. 
[137] E. Björnson, E. De Carvalho, J. H. Sørensen, E. G. Larsson, and P. Popovski, "A random access protocol for pilot allocation in crowded massive MIMO systems," IEEE Trans. Wireless Commun., vol. 16, pp. 2220-2234, Apr. 2017.

[138] R. Couillet and M. Debbah, Random Matrix Methods for Wireless Communications. Cambridge University Press, 2011.

[139] Q. Zhang, S. Jin, K. K. Wong, H. Zhu, and M. Matthaiou, "Power scaling of uplink massive MIMO systems with arbitrary-rank channel means," IEEE J. Sel. Topics Signal Process., vol. 8, pp. 966-981, Oct. 2014.

[140] M. Matthaiou, P. J. Smith, H. Q. Ngo, and H. Tataria, “Does massive MIMO fail in Ricean channels?," IEEE Wireless Commun. Lett., vol. 8, no. 1, pp. 61-64, 2019.

[141] H. Tataria, P.J. Smith, M. Matthaiou, and P. A. Dmochowski, "Uplink analysis of large MU-MIMO systems with space-constrained arrays in Ricean fading," in Proc. IEEE ICC, pp. 1-7, May 2017.

[142] J. Li and Y. Zhao, "Measurement-based asymptotic user orthogonality analysis and modelling for massive MIMO," IEEE Commun. Lett., vol. 21, pp. 2762-2765, Dec. 2017.

[143] J. Hoydis, C. Hoek, T. Wild, and S. ten Brink, "Channel measurements for large antenna arrays," in Proc. IEEE ISWCS, pp. 811-815, Aug. 2012.

[144] X. Wu, N. C. Beaulieu, and D. Liu, "On favorable propagation in massive MIMO systems and different antenna configurations," IEEE Access, vol. 5, pp. 5578-5593, 2017. 
[145] Z. Gao, L. Dai, C. Yuen, and Z. Wang, "Asymptotic orthogonality analysis of time-domain sparse massive MIMO channels," IEEE Commun. Lett., vol. 19, pp. 1826-1829, Oct. 2015.

[146] M. Roy, S. Paquelet, L. L. Magoarou, and M. Crussière, "MIMO channel hardening: A physical model based analysis," arXiv preprint arXiv:1804.07491, 2018.

[147] J. Zhang, L. Tian, R. Xu, Z. Zhang, and J. Zhang, "Favorable propagation with practical angle distributions for mmWave massive MIMO systems," in Proc. IEEE ICC, May 2019.

[148] S. Buzzi and C. D'Andrea, "Energy efficiency and asymptotic performance evaluation of beamforming structures in doubly massive MIMO mmWave systems," IEEE Trans. Green Commun. Netw, vol. 2, pp. 385396, Jan. 2018.

[149] S. Li, P. J. Smith, P. A. Dmochowski, H. Tataria, M. Matthaiou, and J. Yin, “Massive MIMO for Ray-based Channels," in Proc. IEEE ICC, May 2019.

[150] H. Stark and J. W. Woods, Probability, Random Processes, and Estimation Theory for Engineers. Prentice-Hall, Inc., 1986.

[151] H. Tataria and F. Tufvesson, "Impact of spatial consistency on dynamic beamforming for millimeter-wave cellular systems," in Proc. Baltic URSI Symposium, pp. 1-9, Jan. 2019.

[152] S. Wang, B. R. Jackson, and R. Inkol, "Performance characterization of AOA geolocation systems using the Von Mises distribution," in Proc. IEEE VTC Fall, pp. 1-5, Sep. 2012. 
[153] S. Gunnarsson, J. Flordelis, L. Van der Perre, and F. Tufvesson, "Channel hardening in massive MIMO-A measurement based analysis," in Proc. IEEE SPAWC, June 2018.

[154] 3GPP, "Study on channel model for frequencies from 0.5 to $100 \mathrm{GHz}$," Tech. Rep. TR 38.901 (V14.0.0), 3rd Generation Partnership Project (3GPP), Mar. 2017.

[155] M. J. Lighthill, An Introduction to Fourier Analysis and Generalised Functions. Cambridge University Press, 1958.

[156] R. Larson and B. H. Edwards, Calculus. Cengage Learning, 2009. 